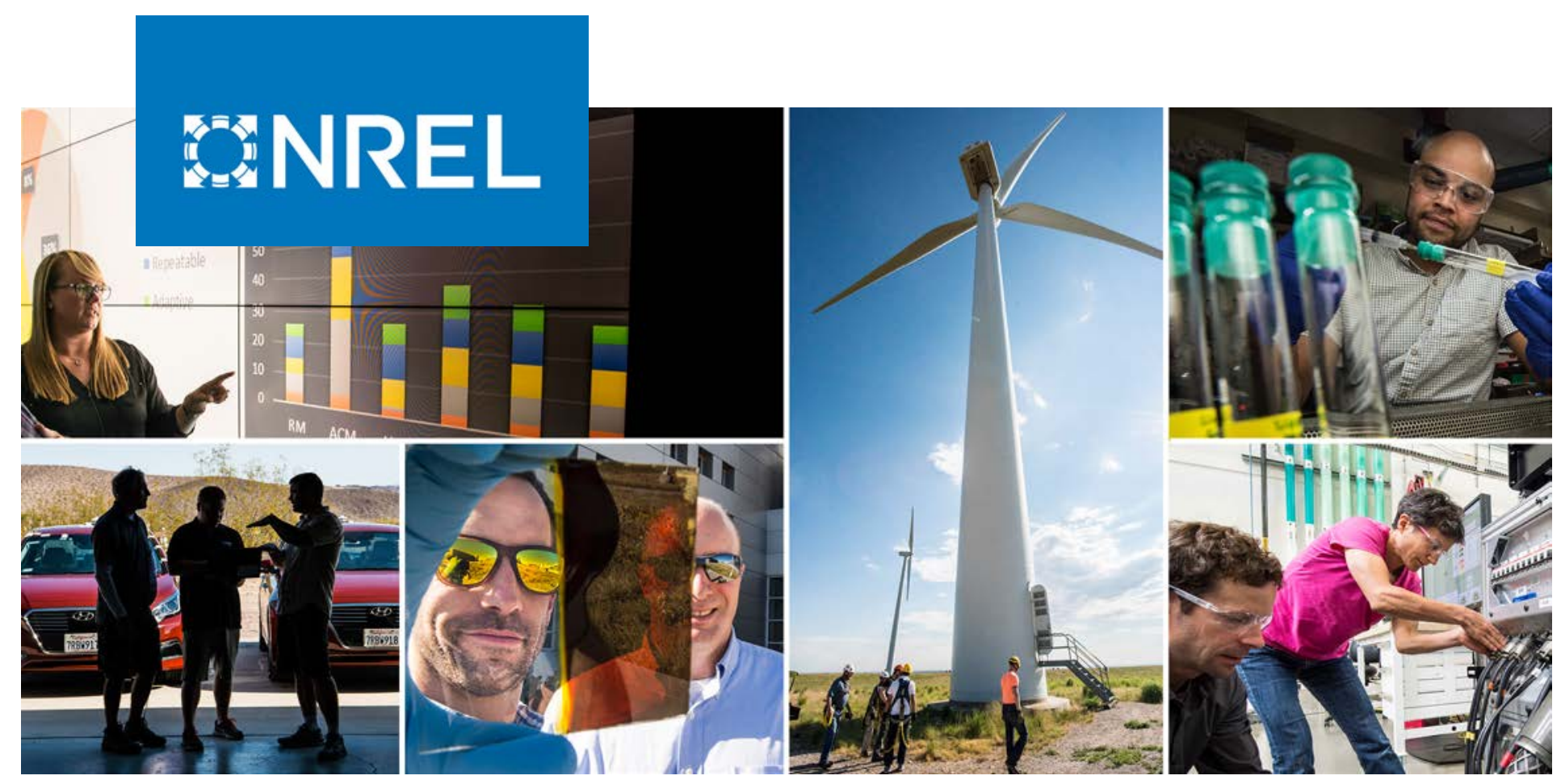

\title{
Process Design and Economics for the Conversion of Lignocellulosic Biomass to Hydrocarbon Fuels and Coproducts: 2018 Biochemical Design Case Update
}

Biochemical Deconstruction and Conversion of Biomass to Fuels and Products via Integrated Biorefinery Pathways

Ryan Davis ${ }^{1}$, Nicholas Grundl ${ }^{1}$, Ling Tao ${ }^{1}$, Mary J. Biddy ${ }^{1}$, Eric C. D. Tan ${ }^{1}$, Gregg T. Beckham ${ }^{1}$, David Humbird ${ }^{2}$, David N. Thompson ${ }^{3}$, and Mohammad S. Roni ${ }^{3}$

1 National Renewable Energy Laboratory

2 DWH Process Consulting

3 Idaho National Laboratory

NREL is a national laboratory of the U.S. Department of Energy Office of Energy Efficiency \& Renewable Energy

Operated by the Alliance for Sustainable Energy, LLC

This report is available at no cost from the National Renewable Energy Laboratory (NREL) at www.nrel.gov/publications.
Technical Report

NREL/TP-5100-71949

November 2018 


\section{EAREL}

\section{Process Design and Economics for the Conversion of Lignocellulosic Biomass to Hydrocarbon Fuels and Coproducts: 2018 Biochemical Design Case Update}

\section{Biochemical Deconstruction and Conversion of Biomass to Fuels and Products via Integrated Biorefinery Pathways}

Ryan Davis ${ }^{1}$, Nicholas Grundl ${ }^{1}$, Ling Tao ${ }^{1}$, Mary J. Biddy ${ }^{1}$, Eric C. D. Tan ${ }^{1}$, Gregg T. Beckham ${ }^{1}$, David Humbird ${ }^{2}$, David N. Thompson ${ }^{3}$, and Mohammad S. Roni ${ }^{3}$

1 National Renewable Energy Laboratory

2 DWH Process Consulting

3 Idaho National Laboratory

\section{Suggested Citation}

Ryan Davis, Nicholas Grundl, Ling Tao, Mary J. Biddy, Eric C.D. Tan, Gregg T. Beckham, David Humbird, David N. Thompson, and Mohammad S. Roni. 2018. Process Design and Economics for the Conversion of Lignocellulosic Biomass to Hydrocarbon Fuels and Coproducts: 2018 Biochemical Design Case Update: Biochemical Deconstruction and Conversion of Biomass to Fuels and Products via Integrated Biorefinery Pathways. Golden, CO: National Renewable Energy Laboratory. NREL/TP-5100-71949. https://www.nrel.gov/docs/fy19osti/71949.pdf.

NREL is a national laboratory of the U.S. Department of Energy Office of Energy Efficiency \& Renewable Energy Operated by the Alliance for Sustainable Energy, LLC

This report is available at no cost from the National Renewable Energy Laboratory (NREL) at www.nrel.gov/publications.

Contract No. DE-AC36-08GO28308
Technical Report NREL/TP-5100-71949 November 2018

National Renewable Energy Laboratory 15013 Denver West Parkway Golden, CO 80401 303-275-3000 • www.nrel.gov 


\section{NOTICE}

This work was authored in part by the National Renewable Energy Laboratory, operated by Alliance for Sustainable Energy, LLC, for the U.S. Department of Energy (DOE) under Contract No. DE-AC36-08GO28308. Funding provided by U.S. Department of Energy Office of Energy Efficiency and Renewable Energy Bioenergy Technologies Office. The views expressed herein do not necessarily represent the views of the DOE or the U.S. Government.

This report is available at no cost from the National Renewable Energy Laboratory (NREL) at www.nrel.gov/publications.

U.S. Department of Energy (DOE) reports produced after 1991 and a growing number of pre-1991 documents are available free via www.OSTI.gov.

Cover Photos by Dennis Schroeder: (clockwise, left to right) NREL 51934, NREL 45897, NREL 42160, NREL 45891, NREL 48097, NREL 46526.

NREL prints on paper that contains recycled content. 


\section{Executive Summary}

The U.S. Department of Energy (DOE) promotes the production of an array of liquid fuels and bio-derived chemicals from lignocellulosic biomass feedstocks by funding fundamental and applied research that advances the state of technology in biomass collection, conversion, and sustainability. As part of its involvement in this program, the National Renewable Energy Laboratory (NREL) investigates the conceptual production economics of these fuels.

Over the past decade, NREL conducted a campaign to quantify the economic implications associated with observed and future targeted performance for the biochemical conversion of corn stover to ethanol through techno-economic modeling. This effort served to set "state of technology" benchmarks and to guide research and development by setting cost targets and tracking progress toward final achievement of these targets in 2012. Beginning in 2013, NREL began transitioning from the singular focus on ethanol to a broad slate of products and conversion pathways, generally focusing on drop-in hydrocarbon fuels or fuel blendstocks, ultimately to establish similar benchmarking and targeting efforts. Several earlier technical reports were released over 2013-2015 documenting initial strategies for achieving interim cost projections based either on biological or catalytic upgrading of lignocellulosic sugars, but with less quantitative focus on longer-term projections for ultimately achieving final hydrocarbon fuel cost goals. This report serves as an update to the biological sugar conversion approach, reflecting modifications to underlying conversion operational strategies, as well as refinements to the techno-economic model details. In addition, the report includes a more quantitative focus on envisioned processing requirements for achieving final fuel cost goals moving further into the future, via inclusion of value-added coproducts.

The overarching process designs evaluated here convert biomass to diesel- and naphtha-range fuels using alkaline and mechanical refining pretreatment, enzymatic saccharification, biological (fermentative) conversion of hydrolysate sugars to intermediate fuel precursors, and catalytic upgrading of those intermediates to final fuel products. Additionally, value-added coproductsrepresented by adipic acid as a proof-of-concept example - are produced by the deconstruction and upgrading of lignin and other biomass residual components through a similar sequential biological and catalytic processing train. Ancillary areas - feed handling, hydrolysate processing, wastewater treatment, residual waste combustion, and utilities - are also included in the design. Broadly, the fuel production processes considered in this report are based on two example anaerobic pathway classes for bioconversion of hydrolysate sugars to hydrocarbon fuel intermediates, namely short-chain carboxylic acids and 2,3-butanediol (BDO), followed by catalytic upgrading steps to remove oxygen and undergo condensation/oligomerization reactions to produce longer-chain hydrocarbon fuel blendstocks. Aerobic bioconversion pathways to fuel components (e.g., lipid/fatty acid pathways) are not included in this design case, given more challenging design and economic constraints for such pathways in ultimately being able to achieve the required cost targets (on the order of roughly $\$ 2$ /gallon gasoline equivalent (GGE) cost premiums previously estimated for aerobic versus anaerobic options). Detailed material and energy balances and capital and operating costs for this baseline process are also documented.

This techno-economic analysis models a production cost for cellulosic hydrocarbon biofuels that can be considered as a baseline to assess the competitiveness and market potential for the technology. It can also be used to quantify the economic impact of individual conversion 
performance targets and prioritize them in terms of their potential to reduce cost. The analysis presented here also includes consideration of key environmental sustainability implications of the modeled biorefineries by tracking sustainability metric indicators such as carbon yields, primary energy import demands (natural gas and power imports), and water consumption attributed to the conversion process models. Additionally, an accounting of energy balances is provided, to quantify the energy output allocations across the biorefinery.

Building on prior design report practices, NREL, supported by subcontractor DWH Process Consulting, performed a feasibility-level analysis for a plausible integrated biorefinery conversion process to meet the ultimate DOE fuel selling price goal of $\$ 2.50 / \mathrm{GGE}$ or less by the year 2030. The modeled biorefinery processes 2,205 dry tons biomass per day at a target price of $\$ 71.26 /$ dry ton (delivered to the pretreatment reactor throat) and achieves a fuel selling price of $\$ 2.49 /$ GGE for the "acids" pathway or $\$ 2.47 /$ GGE for the "BDO" pathway to fuels (2016 U.S. dollars) as determined by modeled conversion targets and " $n$th -plant" project costs and financing. These fuel price estimates are attributed to a total fuel yield of 44.8 and $43.2 \mathrm{GGE} / \mathrm{dry}$ ton for the acids and BDO pathways, respectively, as well as a final adipic acid coproduct yield of 259 and $266 \mathrm{lb} /$ dry ton for the respective cases, with an adipic acid market value of $\$ 0.86 / \mathrm{lb}$. Additionally, given substantial demands for caustic (sodium hydroxide) and acid usage throughout the integrated process at considerable costs, this work highlights the need for either recovering and reusing these chemicals through advanced separations technologies, or otherwise offsetting a portion of those costs through the sale of the resultant sodium sulfate byproduct. The latter is reflected in this report, generating a smaller co-product revenue stream for sale of this salt at $\$ 0.07 / 1 \mathrm{~b}$, beyond the purposeful co-production of adipic acid. All modeled fuel prices are also based on underlying financial assumptions including $10 \%$ internal rate of return, $40 \%$ equity financing (60\% debt financing at $8 \%$ interest), and 30-year plant lifetime.

Both pathways exhibit high sensitivity to yields across the fuel production trains, but even more strongly to both yields and productivities for lignin deconstruction and bioconversion to coproducts, given both the high processing costs but also high value and thus revenue garnered from the lignin coproduct train as a key factor in achieving the fuel cost targets stipulated here. For achieving more near-term interim fuel selling price goals of $\$ 3 / \mathrm{GGE}$ by 2022 , simplistically assuming all other conversion process parameters remain fixed (aside from feedstock cost at $\$ 79.07 /$ dry ton for an interim 2022 target), the overall adipic acid coproduct yields could be relaxed by roughly $12 \%$ to 229 and $235 \mathrm{lb}$ /dry ton for acids and BDO, respectively. Finally, the report includes a brief discussion on additional opportunities for future consideration that may further improve biorefinery economics, primarily alternative configurations for conversion of both carbohydrates and lignin to either fuels or products as well as opportunities for waste gaseous carbon utilization to improve overall carbon retention efficiencies. 


\section{Biological Renewable Diesel Blendstock (RDB) via Butyric Acids: Process Engineering Analysis DMR Pretreatment, Continuous Enzymatic Hydrolysis, Anaerobic Bioconversion, Catalytic Upgrading, Lignin Conversion to Coproducts \\ All Values in $2016 \$$ \\ Minimum Fuel Selling Price (MFSP, Gasoline-Equivalent \\ Basis): \\ Contributions: Feedstock Fuel Conversion \\ Coproduct Conversion \\ Fuel Production \\ Fuel Yield \\ Adipic Acid Coproduct Yield \\ Butyric Acid Process Yield [Theoretical Yield] \\ Feedstock + Handling Cost \\ Internal Rate of Return (After-Tax) \\ Equity Percent of Total Investment \\ \$2.49 /GGE

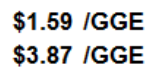

$\$ 1.59$ /GGE

$\$ 3.87$ /GGE \\ $-\$ 2.97 / G G E$ \\ 32.5 MMGGE per year \\ 44.8 GGE / dry U.S. ton feedstock \\ $259 \mathrm{lb} /$ dry U.S. ton feedstock \\ 0.43 [0.49] kg Acid $/ \mathrm{kg}$ Total Sugars ( $89 \%$ of theoretical) \\ $\$ 71.26$ /dry U.S. ton feedstock \\ $10 \%$ \\ $40 \%$}

\begin{tabular}{|c|c|}
\hline \multicolumn{2}{|l|}{ Capital Costs } \\
\hline Area 200: Pretreatment & $\$ 48,653,825$ \\
\hline Area 300: Sugar Hydrolysis and Conditioning & $\$ 62,697,299$ \\
\hline Area 400: Enzyme Production & $\$ 11,526,084$ \\
\hline Area 500: Bioconversion and Upgrading & $\$ 43,799,229$ \\
\hline Area 600: Wastewater & $\$ 38,810,731$ \\
\hline Area 700: Lignin & $\$ 127,474,183$ \\
\hline Area 800: Boiler & $\$ 48,720,730$ \\
\hline Area 900: Utilities \& Storage & $\$ 17,195,641$ \\
\hline Total Installed Equipment Cost & $\$ 398,877,721$ \\
\hline Added Direct + Indirect Costs & $\$ 359,522,279$ \\
\hline$(\%$ of $\mathrm{TCl})$ & $47 \%$ \\
\hline Total Capital Investment (TCI) & $\$ 758,400,000$ \\
\hline Installed Equipment Cost/Annual GGE & $\$ 12.28$ \\
\hline Total Capital Investment/Annual GGE & $\$ 23.35$ \\
\hline Operating Hours Per Year (On-Stream Factor) & $7884(90 \%)$ \\
\hline Loan Rate & $8.0 \%$ \\
\hline Term (years) & 10 \\
\hline Capital Charge Factor (Computed) & 0.129 \\
\hline Fuel Carbon Retention Efficiencies: & \\
\hline From Hydrolysate Sugar (Fuel C / Sugar C) & $49.9 \%$ \\
\hline From Biomass (Fuel C / Biomass C) & $26.2 \%$ \\
\hline Adipic Acid Carbon Efficiency from Biomass & $14.4 \%$ \\
\hline Maximum Yields $(100 \% \text { of Theoretical })^{a}$ & \\
\hline Fuel Production (U.S. ton/yr) & 128,798 \\
\hline Current Fuel Production (U.S. ton/yr) ${ }^{\mathrm{b}}$ & 99,659 \\
\hline Current Yield (Actual/Theoretical) & $77.4 \%$ \\
\hline${ }^{a}$ Complete conversion of biomass carbohydrat & \\
\hline $\begin{array}{l}{ }^{b} \text { Recovered fuel yield after concentration, sen } \\
\text { (Theoretical yields above do not consider p } \\
\text { losses during product upgrading and purific }\end{array}$ & $\begin{array}{l}\text { sydrotreating } \\
\text { ss recovery }\end{array}$ \\
\hline
\end{tabular}

Manufacturing Costs (cents/GGE fuel product)

Feedstock + Handling 158.9

Sulfuric Acid $\quad 24.2$

Caustic $\quad 105.1$

Glucose (enzyme production) $\quad 26.0$

Hydrogen $\quad 0.0$

Electricity (import) $\quad 72.9$

Other Raw Materials $\quad 36.0$

Catalysts $\quad 5.1$

Waste Disposal

$\mathrm{Na} 2 \mathrm{SO} 4$ Coproduct $(\$ 0.07 / \mathrm{lb}) \quad-52.4$

Adipic Acid coproduct $(\$ 0.86 / \mathrm{lb}) \quad-493.1$

Fixed Costs $\quad 61.5$

Capital Depreciation $\quad 73.9$

Average Income Tax $\quad 22.7$

\begin{tabular}{ll} 
Average Return on Investment & 204.0 \\
\hline Total & 249.0
\end{tabular}

\begin{tabular}{ll} 
Manufacturing Costs (\$/yr) & \\
\hline Feedstock + Handling & $\$ 51,600,000$
\end{tabular}

$\begin{array}{lr}\text { Feedstock + Handling } & \$ 51,600,000 \\ \text { Sulfuric Acid } & \$ 7,900,000\end{array}$

Caustic \$34,100,000

Glucose (enzyme production) $\quad \$ 8,400,000$

Hydrogen $\$ 0$

Electricity (import) $\$ 23,700,000$

Other Raw Materials $\$ \$ 11,700,000$

Catalysts $\quad \$ 1,700,000$

Waste Disposal $\$ 1,400,000$

Na2SO4 Coproduct $(\$ 0.07 / \mathrm{lb}) \quad-\$ 17,000,000$

Adipic Acid coproduct $(\$ 0.86 / \mathrm{lb}) \quad-\$ 160,100,000$

Fixed Costs $\$ 20,000,000$

Capital Depreciation $\$ 24,000,000$

Average Income Tax $\quad \$ 7,400,000$

Average Return on Investment $\$ 66,200,000$

Total $\$ 81,000,000$

Specific Operating Conditions

Enzyme Loading (mg/g cellulose) 10.0

Net Electricity Import (kWh/GGE) 10.9

Plant Electricity Use (kWh/GGE) 15.8

Metabolic Yield ( $\mathrm{g} / \mathrm{g}$ substrate)

Glucose to Butyric $\quad 0.465$

Glucose to Biomass $\quad 0.041$

Xylose to Butyric $\quad 0.416$

Xylose to Biomass $\quad 0.123$

Arabinose to Butyric $\quad 0.418$

Arabinose to Biomass $\quad 0.016$

Figure ES-1. Economic summary for anaerobic bioconversion via acids pathway 


\section{Biological Renewable Diesel Blendstock (RDB) via 2,3-Butanediol: Process Engineering Analysis DMR Pretreatment, Whole-slurry Enzymatic Hydrolysis \& Anaerobic Bioconversion, Catalytic Upgrading, Lignin Conversion to Coproducts \\ All Values in 2016\$ \\ Minimum Fuel Selling Price (MFSP, Gasoline-Equivalent \\ Basis): \\ Contributions: Feedstock \\ Fuel Conversion \\ Coproduct Conversion \\ Fuel Production \\ Fuel Yield \\ Adipic Acid Coproduct Yield \\ 2,3-Butanediol Process Yield [Theoretical Yield] \\ Feedstock + Handling Cost Internal Rate of Return (After-Tax) \\ Equity Percent of Total Investment \\ $\$ 2.47$ /GGE \\ $\$ 1.65$ /GGE \\ $\$ 3.83 / \mathrm{GGE}$ \\ $-\$ 3.00 / G G E$ \\ 31.3 MMGGE per year (at $68^{\circ} \mathrm{F}$ ) \\ 43.2 GGE / dry U.S. ton feedstock \\ $266 \mathrm{lb} /$ dry U.S. ton feedstock \\ $0.45[0.5] \mathrm{kg} \mathrm{BDO} / \mathrm{kg}$ Total Sugars (90\% of theoretical) \\ $\$ 71.26$ /dry U S ton feedstock \\ $10 \%$ \\ $40 \%$}

\begin{tabular}{|c|c|}
\hline \multicolumn{2}{|l|}{ Capital Costs } \\
\hline Area 200: Pretreatment & $\$ 48,653,825$ \\
\hline Area 300: Sugar Hydrolysis and Conditioning & $\$ 15,441,726$ \\
\hline Area 400: Enzyme Production & $\$ 11,526,084$ \\
\hline Area 500: Bioconversion and Upgrading & $\$ 61,083,902$ \\
\hline Area 600: Wastewater & $\$ 35,611,508$ \\
\hline Area 700: Lignin & $\$ 128,436,573$ \\
\hline Area 800: Boiler & $\$ 50,985,372$ \\
\hline Area 900: Utilities \& Storage & $\$ 15,506,286$ \\
\hline Total Installed Equipment Cost & $\$ 367,245,276$ \\
\hline Added Direct + Indirect Costs & $\$ 329,554,724$ \\
\hline$(\%$ of $\mathrm{TCl})$ & $47 \%$ \\
\hline Total Capital Investment (TCI) & $\$ 696,800,000$ \\
\hline Installed Equipment Cost/Annual GGE & $\$ 11.74$ \\
\hline Total Capital Investment/Annual GGE & $\$ 22.27$ \\
\hline Operating Hours Per Year (On-Stream Factor) & $7884(90 \%)$ \\
\hline Loan Rate & $8.0 \%$ \\
\hline Term (years) & 10 \\
\hline Capital Charge Factor (Computed) & 0.129 \\
\hline Fuel Carbon Retention Efficiencies: & \\
\hline From Hydrolysate Sugar (Fuel C / Sugar C) & $50.6 \%$ \\
\hline From Biomass (Fuel C / Biomass C) & $25.0 \%$ \\
\hline Adipic Acid Carbon Efficiency from Biomass & $14.8 \%$ \\
\hline Maximum Yields ( $100 \%$ of Theoretical) ${ }^{\mathrm{a}}$ & \\
\hline Fuel Production (U.S. ton/yr) & 151,073 \\
\hline Current Fuel Production (U.S. ton/yr) ${ }^{\mathrm{b}}$ & 95,888 \\
\hline Current Yield (Actual/Theoretical) & $63.5 \%$ \\
\hline${ }^{a}$ Complete conversion of biomass carbohydrat & \\
\hline $\begin{array}{l}{ }^{b} \text { Recovered fuel yield after concentration, sent } \\
\text { (Theoretical yields above do not consider pr } \\
\text { losses during product upgrading and purific }\end{array}$ & $\begin{array}{l}\text { ydrotreating } \\
\text { ss recovery }\end{array}$ \\
\hline
\end{tabular}

Manufacturing Costs (cents/GGE fuel product)

Feedstock + Handling 164.9

Sulfuric Acid $\quad 25.9$

Caustic $\quad 112.5$

Glucose (enzyme production) 27.0

Hydrogen 33.1

Electricity (import) $\quad 71.4$

Other Raw Materials $\quad 33.7$

Catalysts $\quad 9.4$

Waste Disposal 4.5

Na2SO4 Coproduct $(\$ 0.07 / \mathrm{lb}) \quad-55.6$

Adipic Acid coproduct $(\$ 0.86 / \mathrm{lb}) \quad-527.1$

Fixed Costs $\quad 59.7$

Capital Depreciation 706

Average Income Tax 21.8

Average Return on Investment $\quad 195.2$

Total 247.1

Manufacturing Costs $(\$ / y r)$

Feedstock + Handling $\$ 51,600,000$

Sulfuric Acid $\$ \$ 8,100,000$

Caustic $\$ 35,200,000$

Glucose (enzyme production) $\quad \$ 8,400,000$

Hydrogen $\$ 10,400,000$

Electricity (import) \$22,300,000

Other Raw Materials $\quad \$ 10,500,000$

Catalysts $\$ \$ 2,900,000$

Waste Disposal $\$ \$ 1,400,000$

Na2SO4 Coproduct $(\$ 0.07 / \mathrm{lb}) \quad-\$ 17,400,000$

Adipic Acid coproduct $(\$ 0.86 / \mathrm{lb}) \quad-\$ 164,900,000$

Fixed Costs $\$ \$ 18,700,000$

Capital Depreciation $\$ 22,100,000$

Average Income Tax $\$ \$ 6,800,000$

Average Return on Investment $\$ \$ 61,100,000$

Total $\$ 77,200,000$

Specific Operating Conditions

$\begin{array}{ll}\text { Enzyme Loading (mg/g cellulose) } & 10.0 \\ \text { Net Electricity Import (kWh/GGE) } & 10.7 \\ \text { Plant Electricity Use (kWh/GGE) } & 13.7 \\ \text { Metabolic Yield (g/g substrate) } & \\ \quad \text { Glucose to 2,3-BDO } & 0.485 \\ \text { Glucose to Biomass } & 0.017 \\ \text { Xylose to 2,3-BDO } & 0.487 \\ \text { Xylose to Biomass } & 0.017 \\ \text { Arabinose to 2,3-BDO } & 0.478 \\ \text { Arabinose to Biomass } & 0.018\end{array}$

Figure ES-2. Economic summary for anaerobic bioconversion via BDO pathway 


\section{Nomenclature}

ACCE Aspen Capital Cost Estimator

$\mathrm{ACM}$

APR

BCD

BDO

BETO

BTU

$\mathrm{CEH}$

CHP

CIP

COD

CSL

CSTR

DAP

DDA

DMR

DOE

$\mathrm{EH}$

FGD

FCI

gal

GGE

GHG

HDO

HP

ILM

INL

IRR

IS
Aspen Custom Modeler

aqueous phase reforming

base-catalyzed deconstruction

butanediol

Bioenergy Technologies Office

British thermal unit

continuous enzymatic hydrolysis

combined heat and power

clean-in-place

chemical oxygen demand

corn steep liquor

continuous stirred tank reactor

diammonium phosphate

deacetylation and dilute acid

deacetylation and mechanical

refining

U.S. Department of Energy

enzymatic hydrolysis

flue gas desulfurization

fixed capital investment

gallon

gallon gasoline equivalent

greenhouse gas

hydrodeoxygenation

high-pressure

integrated landscape management

Idaho National Laboratory

internal rate of return

insoluble solids
SBL inside-battery-limits

lb pound

LHV lower heating value

LCA life cycle analysis

MEK methyl ethyl ketone

MFSP minimum fuel selling price

MM million

MVR mechanical vapor recompression

MYPP Multi-Year Program Plan

$\mathrm{NADH}$ nicotinamide adenine dinucleotide

hydride

NG natural gas

NREL National Renewable Energy

Laboratory

ORNL Oak Ridge National Laboratory

OTR oxygen transfer rate

OUR oxygen uptake rate

R\&D research and development

$\mathrm{RCF}$ reductive catalytic fractionation

RO reverse osmosis

SMR steam methane reforming

SOT state of technology

SS soluble solids

TCI total capital investment

TDC total direct cost

TEA techno-economic analysis

TOPO tri-octyl-phosphine oxide

TS total solids

WHSV weight hourly space velocity

WWT wastewater treatment 


\section{Table of Contents}

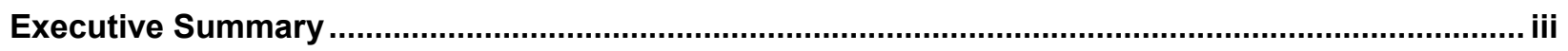

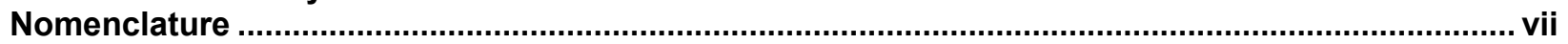

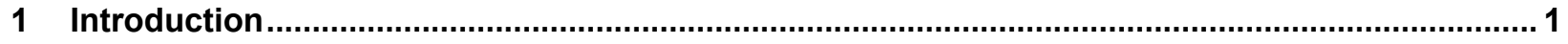

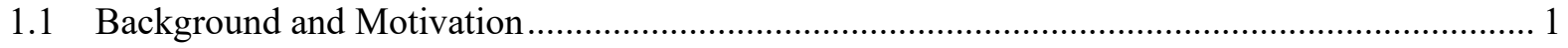

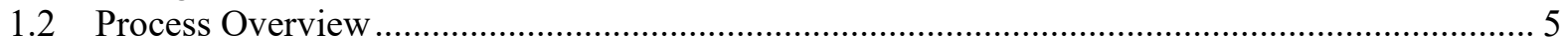

1.3 Techno-Economic Analysis Approach and Assumptions ….................................................. 8

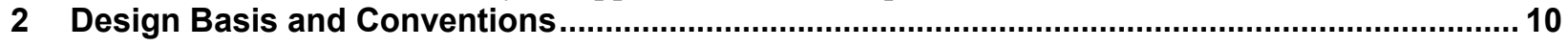

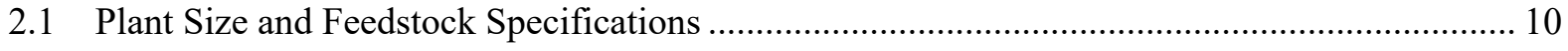

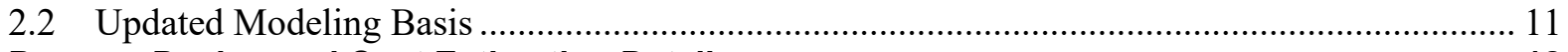

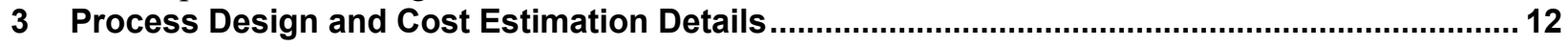

3.1 Area 100: Feedstock Logistics and Handling............................................................... 12

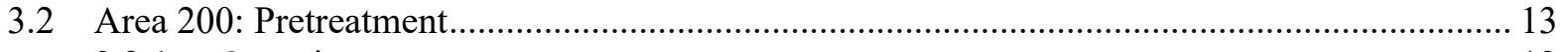

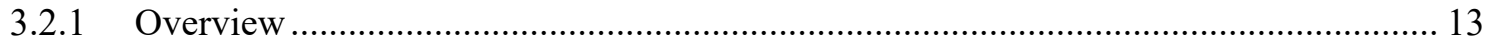

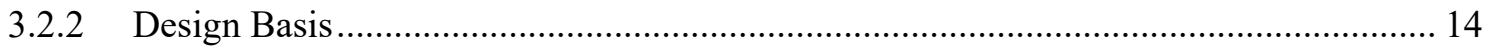

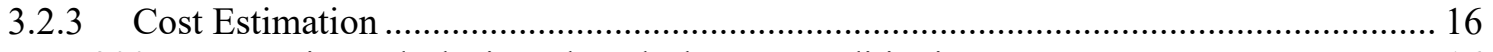

3.3 Area 300: Enzymatic Hydrolysis and Hydrolysate Conditioning .......................................... 16

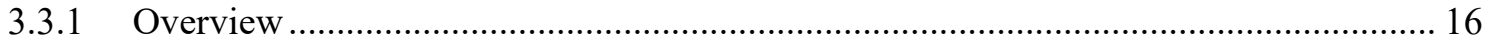

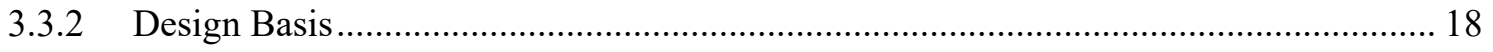

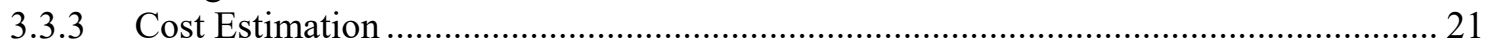

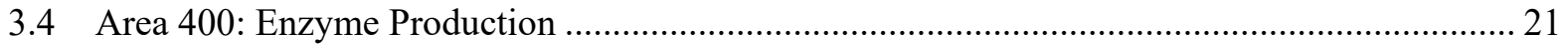

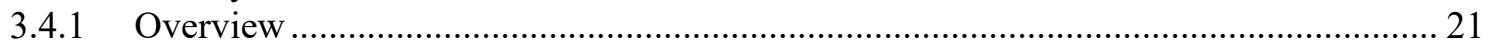

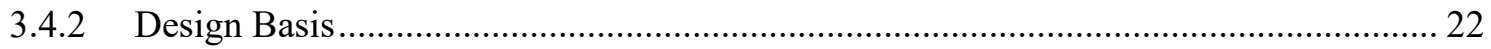

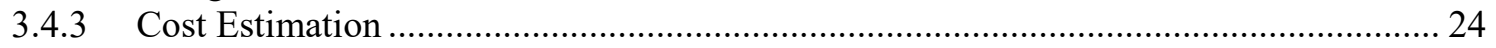

3.5 Area 500: Fermentation, Catalytic Conversion, and Upgrading …..................................... 25

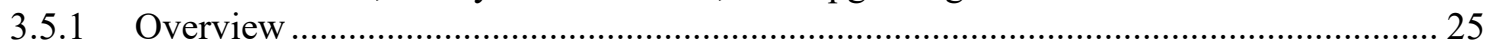

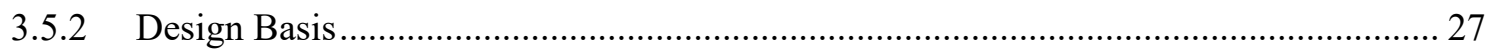

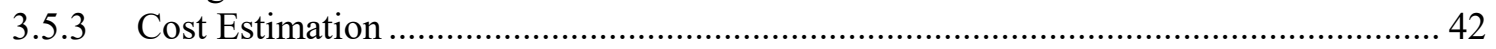

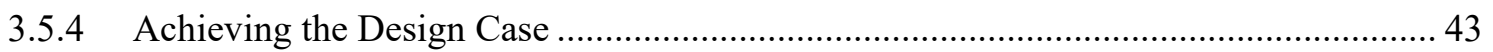

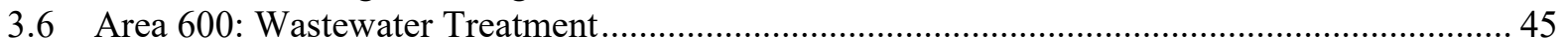

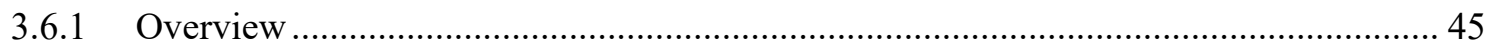

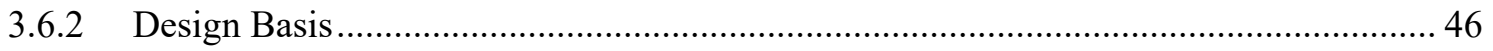

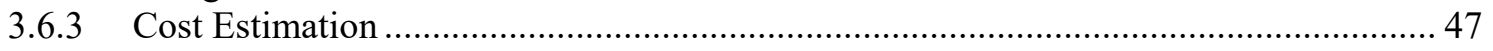

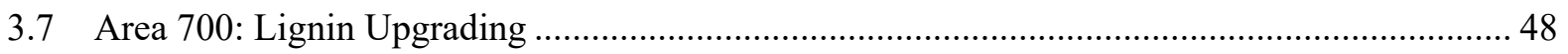

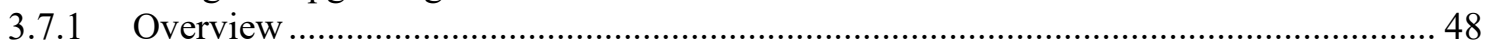

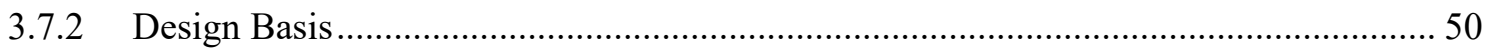

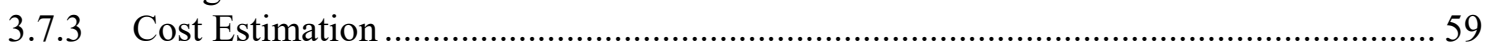

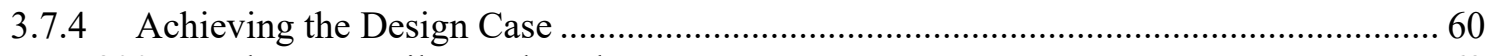

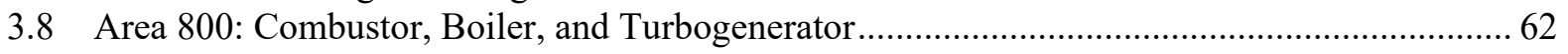

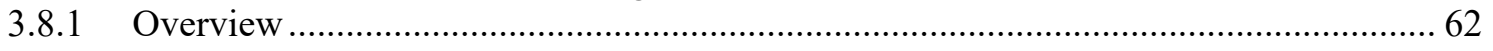

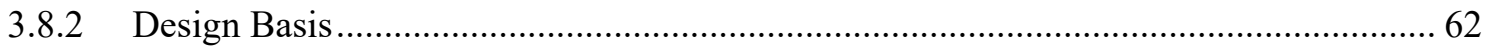

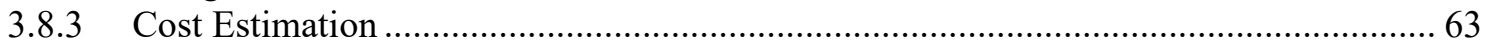

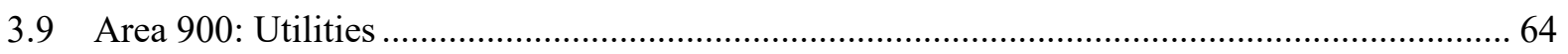

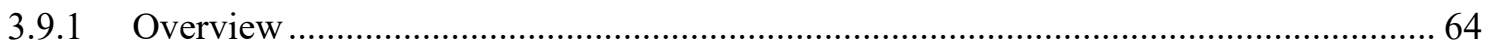

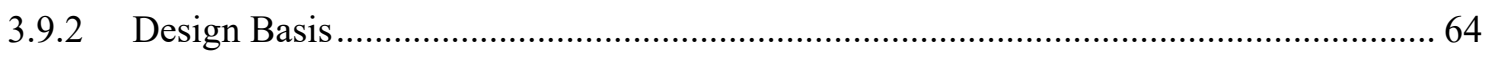

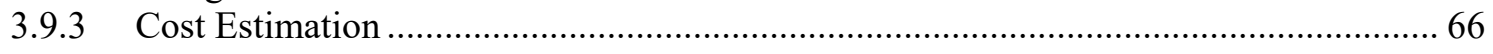

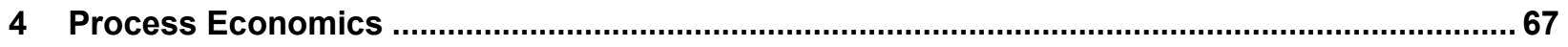

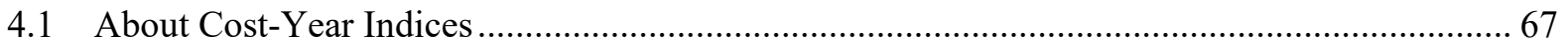

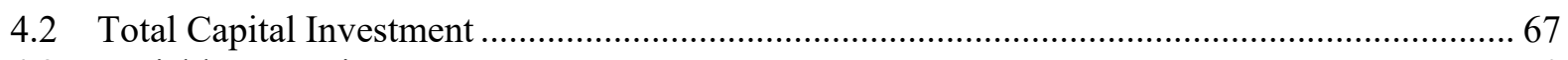

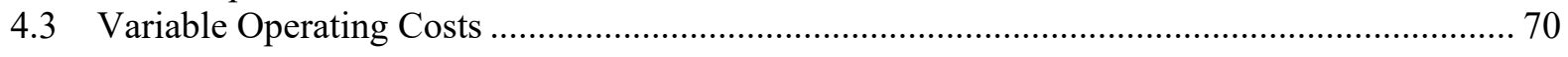




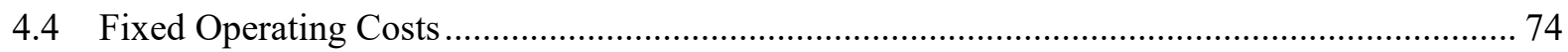

4.5 Discounted Cash Flow Analysis and the Minimum Fuel Selling Price .................................. 75

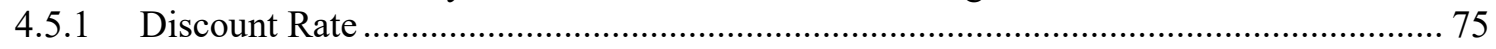

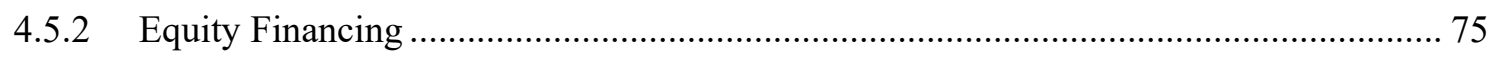

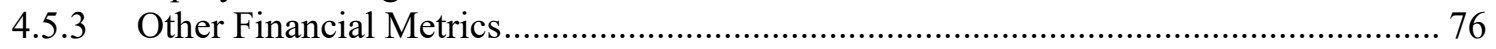

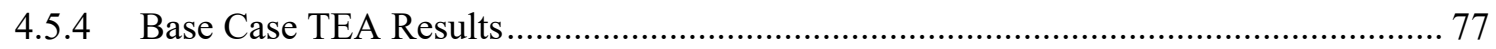

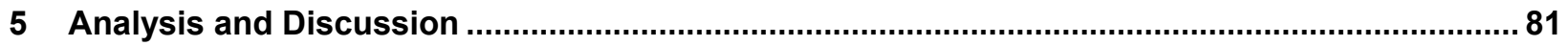

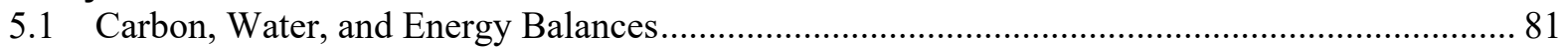

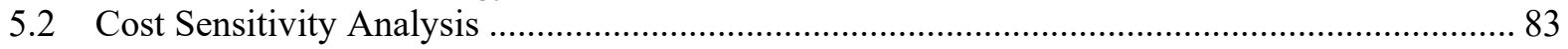

5.2.1 Single-Point Sensitivity Analysis: Acids to Fuels Pathway ....................................... 84

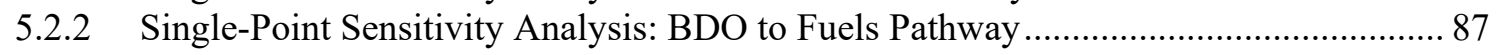

5.2.3 MFSP Sensitivity to Adipic Acid Co-Product Value ............................................... 90

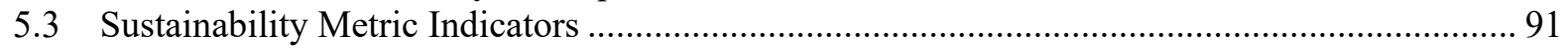

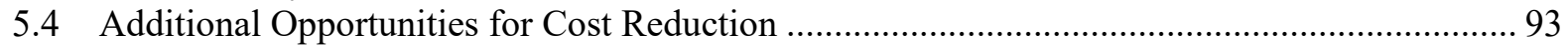

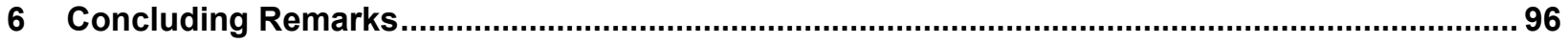

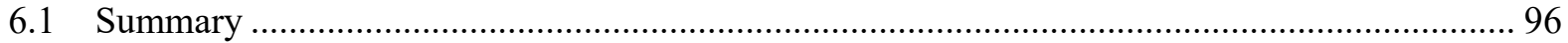

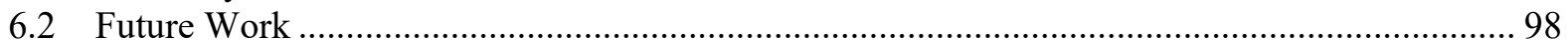

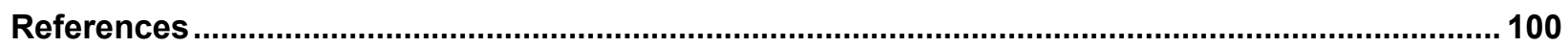

Appendix A. Individual Equipment Costs Summary …............................................................. 114

Appendix B. Discounted Cash Flow Rate of Return Worksheet..................................................... 124

Appendix C. Process Parameters/Operating Summary........................................................... 128

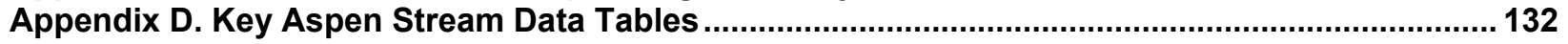




\section{Introduction}

\subsection{Background and Motivation}

The U.S. Department of Energy (DOE) Bioenergy Technologies Office (BETO) promotes the production of liquid fuels from lignocellulosic feedstocks by sponsoring programs in fundamental and applied research that aim to advance the state of biomass conversion technology. These programs include laboratory research to develop improved bioconversion organisms, chemical catalysts, hydrolysis enzymes, and integrated unit operations through synthetic biology, catalyst development and testing, chemical and mechanical pretreatment work, detailed engineering studies of potential processes, and construction of pilot-scale demonstration and production facilities. This research is conducted by national laboratories, universities, and private industry in conjunction with engineering and construction companies.

To support the DOE program, the National Renewable Energy Laboratory (NREL) investigates the process design and economics of modeled cellulosic biorefineries in order to develop a plant gate price for fuels and fuel blendstocks based on process and plant design assumptions consistent with applicable best practices in engineering, construction, and operation. This plant gate price is referred to as the minimum fuel selling price or MFSP. The MFSP can be used by DOE to assess the cost-viability and market penetration potential of a given cellulosic biofuel technology pathway in comparison with petroleum-derived fuels and established biofuel technologies such as starch- or sugar-based ethanol. Ultimately, through techno-economic analysis (TEA), the modeled MFSP may be viewed as a bridge between understanding the process requirements from "bottom-up" modeling that must be achieved in order to meet DOE cost goals set from the "top down."

The TEA effort at NREL also helps to direct biomass conversion research by examining the sensitivity of the MFSP to process alternatives and research advances. Proposed research and its anticipated results can be translated into a new MFSP that can be compared to the benchmark case documented in this report. Such comparison helps to quantify the economic impact of core research targets at NREL, and elsewhere, and to track progress as research evolves through "state of technology" benchmarking of MFSP reductions. It also allows DOE to make more informed decisions about research proposals that claim to reduce MFSP, and to better quantify trade-offs between process metrics (e.g., yields, chemical inputs, and carbon efficiencies), economics (MFSP), and sustainability (e.g., emissions and energy and water usage) among process options for a given technology pathway or amongst multiple pathways.

For more than 20 years, NREL developed design case models and associated reports [1-3] that documented process and cost targets for ethanol production from cellulosic feedstocks via biochemical conversion (e.g., deconstruction to monomeric sugars followed by fermentation to ethanol) based on the best understanding of the technology and equipment costs at the time. As understanding evolved on process and economic metrics, models were refined and rebenchmarked relative to updated targets. The final update to the ethanol design targets was published in 2011 (referred to hereafter as the 2011 design report) [2] and established an MFSP cost goal of \$2.15/gal ethanol (\$3.27/gal gasoline equivalent (GGE), 2007 U.S. dollars) to be achieved in 2012 based on performance data from pilot-scale demonstration runs conducted at NREL. This goal was subsequently achieved through 2012 pilot plant trials, with the pertinent experimental data input to the model, which calculated an ethanol MFSP of \$2.15/gal for a 
commercial-scale " $n^{\text {th }}$-plant" facility processing 2,000 dry metric tons/day of corn stover [4-6]. Further context on the history of NREL's ethanol program, including individual year-by-year modeled production costs, may be found in published NREL reports $[5,7]$.

Following the 2012 ethanol demonstrations, all BETO platforms began to transition in focus toward hydrocarbon fuel and blendstock products (fungible/infrastructure-compatible fuels), including a new pathway MFSP target of \$3/GGE by 2022 [8]. Within the biochemical platform, two pathway design cases were initially established to document possible strategies to achieve future hydrocarbon MFSP goals, based on a biological sugar conversion pathway (via aerobic fatty acid production and upgrading) and a catalytic sugar conversion pathway (via aqueous phase reforming and upgrading of hydrolysate sugars) $[9,10]$. Both cases focused primarily on production of fuels from biomass carbohydrates, which in isolation only stood to achieve MFSPs on the order of roughly $\$ 5 / \mathrm{GGE}$, and as such were viewed as interim projections for a shorter timeframe prior to 2022. Both cases also highlighted that to ultimately achieve $\$ 3 / \mathrm{GGE}$ or lower MFSP targets, it would be necessary to utilize more of the biomass, namely lignin and other under-utilized components, for production of value-added coproducts.

At the time, specific details were not yet well-understood on what such lignin coproduct trains would look like, but a higher-level sensitivity analysis was provided on feasibility TEA estimates for four example coproduct options. The two example components that maintained oxygen in their structures (adipic acid and 1,4-butanediol) were shown to be superior choices both from an economic and environmental sustainability standpoint. This highlighted a key advantage for bioderived products, namely that maintaining oxygen (a) increases overall mass of the coproduct as well as atom efficiency from the starting biomass and (b) allows for less energy-intensive methods to produce such coproducts. This second advantage is due to the presence of oxygen in the starting biomass, as petrochemical routes must undergo costly and energy-demanding synthesis steps to add oxygen from initial oxygen-free petroleum feedstocks [11]. Alongside such coproduct considerations, market size is also a key factor as the coproduct must exceed small niche market volumes in order to avoid saturating existing product markets to support commodity scale deployment of such biorefineries; this is further discussed later in Section 5.2.3 for the coproduct evaluated in this work.

Historically, the primary focus in NREL's biochemical platform has been on biological conversion routes, although catalytic and hybrid approaches have also become more emphasized in recent years under the Chemical Catalysis for Bioenergy Consortium (https://www.chemcatbio.org) and other efforts. Thus, following the initial design cases noted above, the majority of experimental and TEA activities were focused on bioconversion to fuel precursors. NREL's 2013 "biological design report" targeted the production of fatty acids, produced via aerobic bioconversion of sugars, with the assertion that the fatty acids would be made to be secreted from the cell in order to avoid costly intracellular extraction steps [10]. However, once experimental efforts began to take shape after publication of the report, it became apparent that meeting the stipulated targets for fatty acid yields, fermentation productivities, and product secretion would pose major challenges to be achieved in a short timeframe. Accordingly, in 2014 a new process was developed termed the "C5/C6" pathway, whereby hemicellulose sugars (produced following dilute acid pretreatment) were separated from the cellulose and other solids components and fermented to succinic acid as a coproduct in parallel to fuel production from the glucose (cellulose) fraction. This configuration allowed for relaxing the targets for the 
fuel train bioconversion step, instead based on production of oleaginous yeast with intracellular accumulation (and subsequent extraction) of lipids, while still maintaining similar MFSP goals as the prior fuel-only approach enabled by the coproduct revenue from succinic acid [11].

Following establishment of the C5/C6 pathway TEA models, NREL's experimental efforts moved to focus on this concept over the period of 2014-2015. Over this short timeframe, significant progress was demonstrated for both the C6 (fuel) and C5 (succinic acid) processes, which translated to a roughly $\$ 5 / \mathrm{GGE}$ reduction in modeled benchmark state of technology (SOT) MFSPs in that time [12]. However, as this pathway was generally viewed as a short-term strategy to an intermediate cost goal (after which point the process would again be changed to move to final cost targets in the longer-term), at BETO's direction in 2015, the C5/C6 pathway was de-emphasized to begin focusing on such longer-term goals for ultimately demonstrating cost targets into 2022 and beyond. Thus, NREL's TEA efforts were again exercised to understand the requirements in more detail for the technical targets that would be necessary in order to achieve \$3/GGE MFSPs or lower over such timeframes.

Through 2016-2017, the modeling and experimental work initially focused on four representative bioconversion pathway options for conversion of sugars to fuel precursors, namely oleaginous yeast lipids (aerobic), fatty alcohols (aerobic), carboxylic acids (anaerobic), and 2,3butanediol (BDO) (anaerobic/minimal oxygen required for cell redox balancing). TEA screening indicated that all four pathways exhibited the technical potential to achieve \$3/GGE MFSPs, albeit all would require substantial assistance from lignin coproducts (consistent with prior findings noted above), but significantly more so for the aerobic cases relative to anaerobic (Figure 1, where the bottom negative bar represents the coproduct revenues required from lignin inclusive of lignin coproduct processing costs). This was due to inherently lower energy (GGE) yields and higher processing costs for aerobic bioconversion compared to anaerobic, with the latter tied to the costs of delivering and solubilizing oxygen to an aerobic bioreactor [13].

Additional anaerobic options are also possible, most simplistically also including a maintained focus on ethanol as an intermediate for catalytic upgrading to hydrocarbons (in fact ethanol was originally co-produced alongside 2,3-BDO in early Zymomonas engineering efforts, incurring a separate catalytic upgrading train in parallel with BDO [14]). An ethanol-to-hydrocarbon pathway may be envisioned to offer further cost advantages than even acids or BDO, being a more simplistic and established technology (and with higher potential energy yields across fermentation), but for this same reason also may offer less opportunities for new research advancements to be made. Additionally, intermediate molecules such as carboxylic acids and $\mathrm{BDO}$ also offer more versatility in pursuing opportunities for conversion to both fuels and valueadded products, which may allow for more flexibility in operating a biorefinery to focus on fuels or products as conditions dictate. 


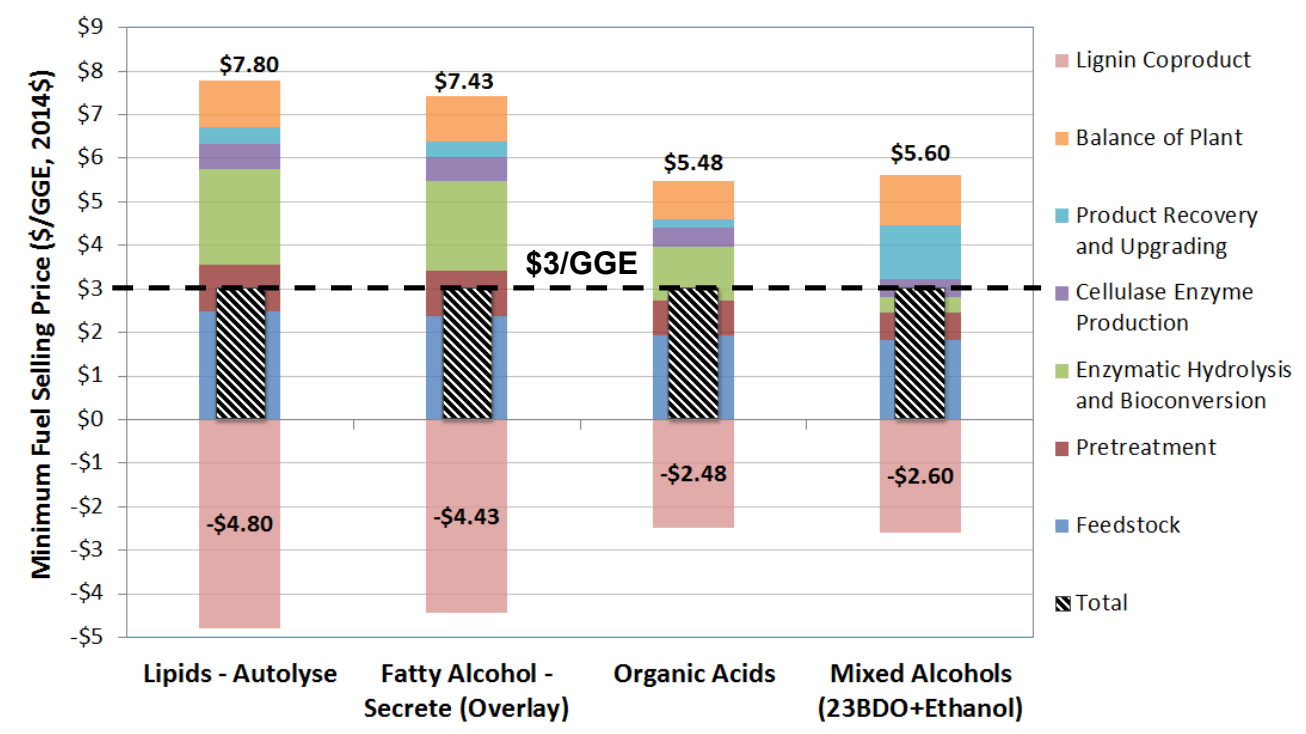

\begin{tabular}{|c|c|c|c|c|}
\hline Metric & Lipids & Fatty Alcohols & Organic Acids & $\mathrm{BDO}+\mathrm{EtOH}$ \\
\hline MFSP (\$/GGE, 2014\$) - prior to coproducts & $\$ 7.80$ & $\$ 7.43$ & $\$ 5.48$ & $\$ 5.60$ \\
\hline Fuel C efficiency from biomass (\%) & $20 \%$ & $21 \%$ & $25 \%$ & $27 \%$ \\
\hline Fuel yield (GGE/ton) & 34.2 & 35.7 & 43.5 & 46.5 \\
\hline TCI (\$MM) - prior to coproducts & $\$ 640$ & $\$ 628$ & $\$ 520$ & $\$ 527$ \\
\hline Fuel carbon chain length & $\sim 14-20$ & $\sim 16-20$ & $\sim 11-14$ & $\sim 8-18$ \\
\hline $\begin{array}{l}\text { Carbon efficiency through lignin-to-coproduct train } \\
\text { required to achieve } \$ 3 / \text { GGE (C in adipic acid vs C } \\
\text { available in residual biomass) }\end{array}$ & $59 \%$ & $56 \%$ & $40 \%$ & $46 \%$ \\
\hline
\end{tabular}

Figure 1. Summary of MFSP and TEA metrics for four representative fuel bioconversion pathways

After reverting back from the C5/C6 pathway to whole-sugar conversion to fuels, from 2015 to 2016, NREL experimental efforts continued focusing primarily on the yeast lipids pathway, at least within the context of integrated biochemical processing. This pathway was chosen because it was the most established case with a history of experimental work on variants of this approach since 2013, relative to other pathways indicated in Figure 1. All other pathways were in earlier stages of research and generally based on clean sugars. Over that time, substantial experimental progress continued to be made, notably the achievement of a roughly $\$ 1.50 / \mathrm{GGE}$ reduction in modeled MFSP in the 2016 SOT for whole sugar conversion relative to 2015 for the C5/C6 pathway. This was achieved by compensating for the loss of the valuable succinic acid coproduct with a significant improvement in enzymatic hydrolysis and lipid fermentation performance including a doubling in lipid fermentation productivity while increasing lipid yield by $26 \%$, which was even further improved in 2017 [12].

Despite these historical achievements, the decision was made in 2018 (preceding this design report) to transition away from the lipids pathway (and aerobic pathways as a whole) in favor of moving to the anaerobic pathway cases shown in Figure 1. This decision was based on the more challenging route to achieve $\$ 3 / \mathrm{GGE}$ by 2022 , which more recently has been further extended to below $\$ 2.50 / \mathrm{GGE}$ by 2030 , in light of the recent decline in oil prices which dictate "cost viability" for biofuels. Such cost targets place significantly more burdens on the requirements from lignin coproducts (or alternative cost reduction needs) to overcome a cost premium of $\$ 2 /$ GGE or more between aerobic versus anaerobic pathways prior to reflecting lignin 
coproducts. Further discussion on the "aerobic versus anaerobic" tradeoffs from a TEA modeling standpoint is included in Section 3.5. In this context, the overall scope of this report focuses on model details attributed to achieving final MFSP costs of $\$ 2.50 / \mathrm{GGE}$ or less by 2030 for both anaerobic fuel train pathways (acids and BDO intermediates), evaluated as two separate process configuration approaches, with additional qualitative discussion at the end of the report on additional process configuration options that may also achieve similar or lower cost targets as may be considered in the future.

\subsection{Process Overview}

As noted above, two separate process cases are considered in this report based on fermentation pathway to the fuel precursor intermediate, namely carboxylic acids and BDO. The pathways share numerous steps in common outside of the fuel fermentation/upgrading operations, but there are also several distinctions to optimize overall process integration, discussed throughout the report. The processes modeled here use deacetylation/mild alkaline extraction and mechanical refining pretreatment of lignocellulosic biomass feedstock (primarily corn stover), followed by enzymatic hydrolysis (saccharification) of the cellulose and xylan, followed by hydrolysate concentration and bioconversion in the acids case or directly to bioconversion in the BDO case. The bioconversion intermediates (acids/BDO) are subsequently catalytically upgraded to final hydrocarbon fuel products. Lignin is deconstructed to soluble monomers and converted (along with other biomass residual components) to muconic acid which is purified and further upgraded to adipic acid for sale as a coproduct. The facility also includes feedstock handling and storage, wastewater treatment (WWT), residual waste combustion, product storage, and utilities. The process is divided into nine areas (Figure 2).

Area 100: Feedstock logistics and handling. The feedstock, in this case blended herbaceous biomass (primarily corn stover), is delivered to the feed handling area from a uniform-format feedstock supply system. All Area 100 processing aspects are outside the scope of this work, and are rolled into delivered feedstock costs at the throat of the pretreatment reactor (Area 200).

Area 200: Pretreatment. In this area, the biomass is processed in a continuous alkaline extraction/deacetylation step to solubilize and remove acetate as well as portions of ash, lignin, and other components (also incurring some losses of carbohydrates). The process utilizes counter-current extraction with recycling of the caustic liquor. The exiting solids are squeezed in a screw press to increase solids content to $30 \mathrm{wt} \%$ and then sent to mechanical refining to open biomass fibers for increased enzyme accessibility through enzymatic hydrolysis.

Area 300: Enzymatic hydrolysis and hydrolysate conditioning. In the BDO case, enzymatic hydrolysis is initiated in a high-solids continuous reactor using a cellulase enzyme produced on site. The partially hydrolyzed slurry is sent to one of several parallel batch reactors. Hydrolysis is completed in the batch reactor with a total time of 5 days between the continuous and batch steps. The whole-slurry hydrolysate is then cooled and directly fermented in the same batch reactors (associated fermentation tankage volume is allocated under A500). In the acids case, the pretreated material is sent through a continuous enzymatic hydrolysis $(\mathrm{CEH})$ process based on a series of hydrolysis reactors each connected through a pump-around loop to filtration units to continuously remove sugars prior to sending residual solids to the next hydrolysis tank. The final remaining hydrolysate stream from the last reactor is sent through a lignin press with the liquor 
combined with the filtrate from the other steps, while the solids fraction is routed to the lignin train. The clarified liquor is concentrated in a mechanical vapor recompression evaporator and then routed to acids fermentation.

Area 400: Enzyme production. An on-site enzyme production section was maintained in this design, consistent with details provided in prior design reports. Purchased glucose (corn syrup) is the primary carbon source for enzyme production, in this case producing enzymes for both cellulose and hemicellulose saccharification. An enzyme-producing fungus (modeled after Trichoderma reesei) is grown aerobically in fed-batch bioreactors. The whole fermentation broth, containing the secreted enzyme, is fed to Area 300 to carry out enzymatic hydrolysis.

Area 500: Fermentation, catalytic conversion, and upgrading. In the BDO case, the hydrolysate is cooled and directly subjugated to whole-slurry fermentation. The fermentation broth is then sent to a lignin press to remove lignin and other insoluble solids, and the clarified stream is routed to aqueous BDO upgrading, yielding butene and minor byproducts. The butene is distilled from the water and then further upgraded through oligomerization and hydrotreating steps to finished hydrocarbon fuels primarily in the diesel range. In the acids case, fermentation is done on clarified hydrolysate in a similar fermentor vessel as used for BDO, but with a pumparound loop through a membrane to isolate acids into a solvent system located on the other side of the membrane (pertractive recovery). The solvent is subsequently recovered and recycled via distillation, and the acids are then upgraded through a series of ketonization, condensation, and hydrotreating steps to hydrocarbon fuels in the diesel range.

Area 600: Wastewater treatment. Wastewater streams (primarily aqueous broth after recovery of fermentation intermediates in Areas 500 and 700) are treated by aerobic digestion and reverse osmosis to remove organics and salts, respectively. Anaerobic digestion is not utilized in this design given substantially lower chemical oxygen demand (COD) loading levels entering Area 600 than in prior cases. The treated water is suitable for recycling and is returned to the process. Sodium sulfate salt recovered from the brine is sold as a secondary coproduct to offset a portion of the caustic and acid demands/costs incurred throughout the integrated design.

Area 700: Lignin upgrading. The black liquor from deacetylation and mechanical refining (DMR) pretreatment is combined with the residual solids separated either during CEH (acids case) or following fermentation (BDO case) and routed to a more severe base-catalyzed deconstruction (BCD) step to solubilize lignin to monomeric components. The lignin monomers, along with other residual (soluble) biomass components, are upgraded through bioconversion to muconic acid, which is then purified and hydrogenated to adipic acid. The adipic acid is recovered through crystallization to a finished coproduct.

Area 800: Combustor, boiler, and turbogenerator. The residual solids, wastewater sludge, and off-gas streams are combusted to generate high-pressure steam for heat and power. Excess steam beyond facility heating demands is converted to electricity for use in the plant.

Area 900: Utilities. This area includes a cooling water system, chilled-water system, process water manifold, and power systems. 


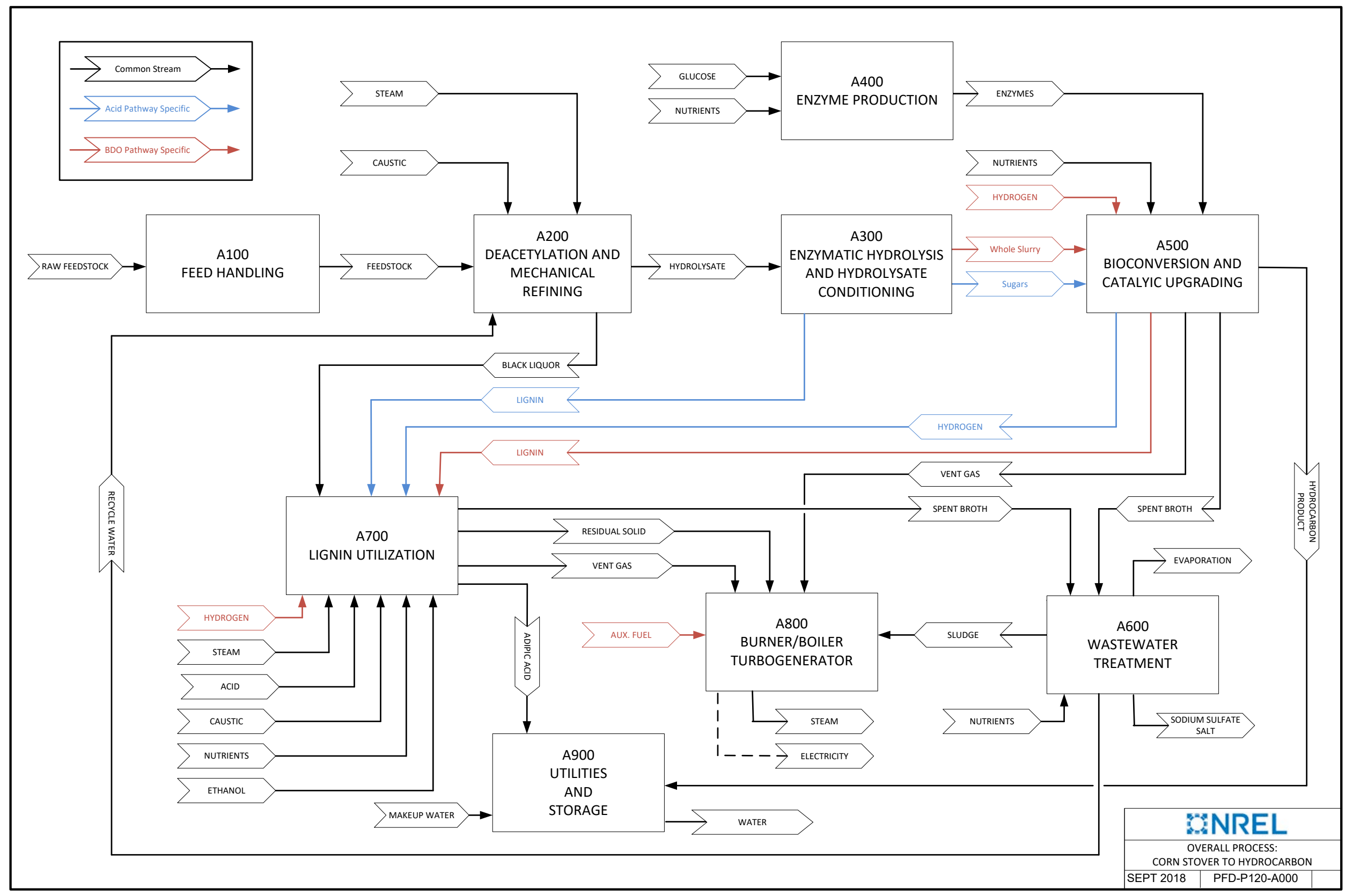

Figure 2. Simplified flow diagram of the overall process. (Key streams only. See Appendix D for more detailed schematic and process flow diagrams.) 


\subsection{Techno-Economic Analysis Approach and Assumptions}

The engineering approach taken in this work for modeling the conversion process is similar to that described in prior design cases, and will not be repeated in the same detail here $[9,10]$. In summary, process flow diagrams are initially developed based on inputs and discussions with researchers around pertinent process configurations (i.e., block flow diagram in Figure 2 and more detailed diagrams provided in Appendix D) from which process simulations are developed in Aspen Plus [15]. This software computes thermodynamically rigorous material and energy balances for each unit operation in this conceptual biorefinery. The material and energy balance data from the Aspen simulation are used to assist in determining the number and size of capital equipment items. As process conditions and flows change, baseline equipment costs are automatically adjusted in an Excel spreadsheet using a scaling factor. These baseline costs come from vendor quotes (a favored procedure when available for larger or nonstandard unit operations and packaged or skid-mounted subsystems), NREL and subcontractor historical cost data, or published engineering literature (when necessary). Final equipment costs for this report are tabulated in Appendix A.

Once equipment costs are determined, direct and indirect overhead cost factors (e.g., installation costs and project contingency) are applied to determine a feasibility-level estimate of total capital investment (TCI) in 2016\$. The TCI, along with the plant operating expenses (also developed using flow rates from the Aspen model), is used in a discounted cash flow rate of return (DCFROR) analysis to determine a plant gate price for total fuel at a given discount rate. The plant gate price, also called the minimum fuel selling price (MFSP), is defined as the sales price (in $\$ / G G E$ ) required to obtain a net present value (NPV) of zero for a 10\% internal rate of return (IRR) after taxes for a hypothetical $n^{\text {th }}$-plant model over a 30-year plant lifetime.

The product of the analysis is a techno-economic model that estimates a rational price for a precommercial process. The resultant MFSP is unique for the set of process conditions simulated, and it should be emphasized that some amount of uncertainty always exists around these chosen conditions, as well as around the assumptions made for capital and raw material costs. The underlying cost estimates and TEA modeling approach generally fall within an "engineering feasibility" level of analysis (generally AACE Class 4 [16]), with an expected uncertainty of approximately $\pm 25 \%$ in the TCI estimates [17] (sensitivity to this and other parameters are considered in Section 5.2). Without a detailed understanding of the basis behind it, the computed MFSP carries a risk of being taken out of context. While the MFSP can be used to assess the marketplace competitiveness of a given process, it is best suited for comparing technological variations against one another or for performing sensitivity analyses that indicate where economic or process performance improvements are needed.

The analysis reported here assumes $n^{\text {th }}$-plant economics. The key assumption implied by $n^{\text {th }}$-plant economics is that our analysis does not describe a pioneer plant; instead, it assumes several plants using the same technology have already been built and are operating. In other words, it reflects a mature future in which a successful industry of $n$ plants has been established. Because the technoeconomic model is primarily a tool for studying new process technologies or integration schemes to comment on their comparative economic impact, $n^{\text {th }}$-plant analysis avoids artificial inflation of project costs associated with risk financing, longer startups, equipment overdesign, and other costs associated with first-of-a-kind or pioneer plants, lest these overshadow the economic impact of the technical research advances in conversion or process integration. While these $n^{\text {th }}$-plant economics may not be 
reflective of pioneer plants and other early technology adopters, they should still help to provide justification and support the underlying technology.

The $n^{\text {th }}$-plant assumptions in the present model apply primarily to the factored cost model used to determine the total capital investment from the purchased equipment cost and to the choices made in plant financing. The $n^{\text {th }}$-plant assumption also applies to some operating parameters, such as an assertion of $90 \%$ process uptime (roughly 329 days operating per year); while some industries such as corn wet milling may operate as high as $96 \%$ uptime, $90 \%$ is viewed here as a reasonable $n^{\text {th }}$-plant target for a relatively complex integrated biorefinery as reflected in this effort. No concessions are included for equipment over-design, beyond standard redundancy included for some equipment such as compressors. These assumptions were agreed upon by BETO and reflect standard metrics applied universally across multiple national laboratory TEA models to allow for a consistent basis for analysis. It should be emphasized, however, that these assumptions carry a degree of uncertainty and are subject to refinement.

While Aspen Plus is thermodynamically rigorous, such detail is not always warranted in the simulation, whether for lack of data or introduction of additional complexity for little gain in accuracy. Some operations, such as membranes and other solid-liquid separations, were modeled as user defined unit operation modules with a fixed performance based on empirical data or by standard engineering practices. Catalytic reactors were modeled using stoichiometric reactions of discrete feed and product components rather than rigorous kinetics or rate expressions, which satisfies mass balances and appropriate reactor sizing when coupled with targeted catalyst space velocities. The Aspen Plus simulation uses component physical properties internal to the software, as well as property data developed at NREL or from the literature $[18,19]$. Similar to prior recent models, the current model does not rely on external property databanks and minimizes the number of custom-defined components within reason (however, the same type of information is still maintained within the model file itself, i.e. no critical information is lost by eliminating use of external databanks). More details on historical components and their modeled properties may be found in prior design reports [2], $[9,10]$. 


\section{Design Basis and Conventions}

\subsection{Plant Size and Feedstock Specifications}

The plant size in the present design is the same as that used in prior designs: 2,205 dry U.S. tons/day (2,000 metric tonne/day). With an expected 7,880 operating hours per year ( $90 \%$ uptime), the annual feedstock requirement is 724,000 dry U.S. tons/year. Given the focus on reducing both feedstock and conversion costs in support of the $\$ 2.50 /$ GGE MFSP goals, NREL and Idaho National Laboratory (INL) conducted initial analyses around feedstock/biorefinery scale, and found that moving to smaller scales (which reduces feedstock transportation costs) continues to be outweighed by biorefinery economy-of-scale penalties, consistent with prior indications from ethanol process modeling work [3]. Thus, at present, the 2,000-dry-metric-tonne/day basis continues to be maintained reflecting $n^{\text {th }}$-plant commercial scale.

Also consistent with prior recent design cases [3, 9, 10], the delivered feedstock composition is left unchanged. This composition was established reflecting previous INL target specifications developed around a uniform-format feedstock supply system, based on blended biomass composed of corn stover and switchgrass [20]. Moving forward, INL's strategies and future targets are evolving with respect to biomass blends and preprocessing logistics in order to further minimize delivered feedstock cost. While it is premature to establish the resulting compositional details of such new strategies (which will be forthcoming in future INL feedstock design cases), at a minimum, the target specifications will be maintained to meet or exceed 59\% carbohydrates and 15.8\% lignin. The feedstock composition assumed in the conversion model is shown in Table 1. In contrast to prior design cases that still focused only on carbohydrates (cellulose and hemicellulose), other components are of similar importance in the present model configurations, notably including lignin and acetate, given the modifications made for inclusion of the ability to upgrade these components to high-value coproducts.

Also of increasing merit in the present design is the component labeled "extractives." The extractives fraction represents the nonstructural component fractions from the compositional analysis, some of which account for analytical mass balance closures below $100 \%$, but otherwise are composed of simple organic components primarily in the form of sugars (e.g., glucose and fructose), sugar alcohols (e.g., glycerol and xylitol), and organic acids (C4-C6 acids) [21]. The amount and type of extractives in the biomass depends on the location and time of harvest, storage/processing logistics, and, in part, how much microbial degradation of the material occurs after harvest; the amount of extractives in a given sample may therefore be indicative of its age. Although the extractives component is thus more variable than other biomass constituents, its largely simple organic disposition makes it more important for the present and future biorefinery designs that are increasingly targeting utilization of the "whole" biomass for economical conversion to products. Thus, this component is also considered for its utility to be used in the lignin-to-coproducts train, rather than being considered a waste and handled in wastewater treatment as in prior design cases (discussed later). Sucrose is another extractive component, but it is measured separately in laboratory analysis and has been added as a separate feedstock component in the present design. The amount of sucrose present in the biomass is dependent on harvesting and handling practices. Although not pursued for economic value, the ash component may also merit further investigation regarding speciation of ash constituents to better understand potential implications on materials of construction (e.g. related to chloride) or to downstream catalyst activity (e.g. ash constituents that may pose high risk for catalyst poisoning). 
Table 1. Delivered Feedstock Composition Assumed in the Present Design

\begin{tabular}{lc}
\hline Component & Composition (dry wt \%) \\
\hline Glucan & 35.1 \\
Xylan & 19.5 \\
Lignin & 15.8 \\
Ash & 4.9 \\
Acetate & 1.8 \\
Protein & 3.1 \\
Extractives & 14.7 \\
Arabinan & 2.4 \\
Galactan & 1.4 \\
Mannan & 0.6 \\
Sucrose & 0.8 \\
\hline Total structural carbohydrate & 59.0 \\
\hline Total structural carbohydrate + sucrose & 59.8 \\
\hline Moisture (bulk wt \%) & 20.0 \\
\hline groups present in the hemicellulose polymer, converted to acetic acid under low-pH conditions.
\end{tabular}

In this design report update, the 2030 target delivered feedstock cost is set to $\$ 71.26 /$ dry U.S. ton (2016\$) based on guidance from partners at INL, reflective of all pre-processing costs through delivery to the "throat of the reactor" in the biorefinery. This represents a roughly $16 \%$ cost reduction relative to prior 2017 targets at $\$ 85.06 /$ ton $(2016 \$$, originally $\$ 84 /$ ton in $2014 \$$ ), which were subsequently met based on INL's 2017 state of technology demonstrations. More specific details attributed to feedstock logistics and future R\&D plans in support of this cost target will be documented through INL feedstock design report updates in the future (this report precedes that from INL), but a brief discussion around general strategies for achieving this target is provided in Section 3.1.

\subsection{Updated Modeling Basis}

In keeping with prior recent design report updates, a number of underlying model parameters are maintained consistently in the present work. These include a 0.5 -year facility startup time and $90 \%$ process uptime (7,884 hours per year on-stream factor). It should be emphasized that these are reflective of the $n^{\text {th }}$-plant modeling basis employed here, as discussed above, and early pioneer plants may require significantly longer startup times or sustain shorter annual uptimes. Other conventions such as reported units and physical quantities, like total solids (TS) and insoluble solids (IS), are consistent with details described previously $[9,10]$.

The results from this analysis are reported primarily in terms of energy yields in gallons gasoline equivalent, e.g., \$/GGE, GGE/yr, and GGE/ton. This includes energy yield in both the diesel and naphtha product cuts from hydrotreating distillation, rather than yield of a single product with the other treated as a coproduct. This is done to maintain focus on total fuel (energy) yield from biomass carbon, to avoid introducing unnecessary subjectivity in decisions for distillation column product cuts, or over-interpreting the current projections for catalytic upgrading steps with regards to catalyst selectivity to diesel over naphtha. Additionally, as the product distributions are different between the two sugar fuel train pathway approaches (acids and BDO), and even can be different among multiple options for intermediate upgrading within a single given pathway, to maintain consistent grounds, all yields and MFSPs are normalized to the GGE basis according to their energy content. Lower heating values (LHVs) for both diesel- and naphtha-range products were calculated by the Aspen model, and correspond to similar values for standard petroleum-equivalent products [22]. To translate to a GGE basis, a conventional gasoline heating value of 116,090 BTU/gal (LHV basis) was applied [22]. 


\section{Process Design and Cost Estimation Details}

\subsection{Area 100: Feedstock Logistics and Handling}

Feedstock logistics include operations that take place after the biomass is produced in a field or forest, but before it is introduced into a conversion process. All activities related to feedstock logistics are directed at reducing the delivered cost of sustainably produced feedstock, improving and preserving the quality of harvested feedstock to meet the needs of biorefineries, and/or expanding the volume of feedstock materials accessible to the bioenergy industry. Feedstock logistics efforts are primarily focused on identifying, developing, demonstrating, and validating efficient and economic systems to harvest, collect, transport, store, and preprocess raw biomass from a variety of crops to reliably deliver high-quality, high-volume, affordable feedstocks to biorefineries. Previous details associated with feedstock logistics, as well as feedstock compositional goals, have been documented in prior design reports [10], and future projection details will be published in a forthcoming design report by INL. This report precedes INL's and we defer to their future report to document the details of future feedstock logistics targets (which are otherwise outside the scope of the present TEA focus on biorefinery conversion); however, here we outline options based on inputs from INL which could further reduce the overall price associated with biomass while still maintaining the desired quality and property requirements to deliver a feedstock ready for the throat of reactor.

Feedstock has a large impact on the overall MFSP of biofuels, thus, reductions in feedstock costs can have a significant role in driving down the costs of biofuels and meeting future MFSP goals below $\$ 2.50 /$ GGE. A reduction in feedstock price to $\$ 71.26 /$ dry short ton $(2016 \$$ ) would support this cost goal. Reducing the feedstock access cost is a key opportunity for further cost reductions. The farm gate cost is composed of the costs of establishing the crop (for perennial energy crops); harvest, collection, and road-siding the biomass; and a "grower payment" that represents the nutrient replacement cost and a grower profit incentive [23]. Opportunities for reducing these costs focus around utilizing more/different types of biomass to allow greater aggregate biomass to be available lower on the supply curve. Utilization of dedicated energy crops with increased yields that can also be managed independently of row-crop commodities allows new, more cost-effective logistics compared prior herbaceous feedstock logistics approaches.

Additionally, cost will be reduced through the utilization of low-cost, low-quality but readily available waste materials such as municipal solid waste (e.g., yard wastes). To utilize these materials, additional preprocessing and/or blending will be incorporated to meet conversion specifications. Improving quality will enable reduced dockage for not meeting conversion specifications and thus lower feedstock costs. Integrated landscape management (ILM) will be considered as both a method of increasing biomass supplies while affording the opportunity to offset grower costs with additional revenue sources. ILM is a promising strategy to reduce grower payments by increasing grower profitability through sustainable production practices, while increasing the availability of biomass by integrating high-yielding energy crops into unprofitable subfields [24]. ILM is increasingly recognized for its potential for sustainable food and energy production and additional benefits of improved ecological functions [25-29].

Another opportunity for lowering feedstock costs is to reduce losses of convertible material during harvest, collection, and storage. Losses in storage are quite high for herbaceous feedstocks in particular, averaging as much as $12 \%$. Storage losses are the primary factor leading to a requirement to purchase $15 \%$ more biomass than reaches the reactor throat. Reducing these losses through approaches 
such as field-side or local depot-located grinding and high-moisture densification is a potential cost trade-off that will be explored. In addition, inventory management strategies will be developed to target the highest moisture biomass for conversion soon after harvest.

Relatively inexpensive preprocessing technologies, such as air classification and size classification, have been shown to be effective for separating lower-quality fractions from the bulk of the biomass [30], as well as for separating individual plant tissues [31,32], and will contribute to the cost reduction by separating out potential higher-value coproduct streams to offset production of biofuel. Fractional milling during continuous grinding processes can be used to separate specific fractions based on the quality and specific use for each fraction [33]. Chemical preprocessing has also been shown to be effective for removing conversion inhibitors [34], which could then be recovered as coproducts. In this way, the carbon efficiency of the overall field-to-biofuel system could be profitably improved.

Intermediately preprocessed biomass fractions could be diverted from the primary conversion system to another system for which the fraction is better suited (for example, solubilizing lignin for routing to the biorefinery lignin train, leaving carbohydrate-enriched biomass to be routed directly to biorefinery pretreatment/hydrolysis). This may allow increased carbon utilization potential within respective fuel and coproduct trains.

Supply system intensification is another area that will be analyzed for the potential for feedstock cost reductions. New strategies that move preprocessing operations to the field, such as size reduction, can reduce both capital and energy costs for feedstock preprocessing at the biorefinery, including the fieldside grinding/densification and forage chopper strategies described above. Opportunities for process intensification in preprocessing operations exist through the integration of drying with size reduction and densification. BETO-funded R\&D has shown that drying requirements are greatly reduced through the use of wet fractional milling and high-moisture densification [35-39]. Further potential cost reductions include utilization of different types of mills and improving drying efficiency of loose biomass and high-moisture pellets using low-temperature drying technologies. Finally, utilization of existing commodity feed handling infrastructure will offer significant cost savings through the use of pelleted or briquetted feedstocks.

\subsection{Area 200: Pretreatment}

\subsubsection{Overview}

Pretreatment is accomplished in the current design by way of deacetylation and mechanical refining (DMR). Here, what is termed "deacetylation" is a somewhat more severe alkaline extraction operation, targeted to also remove more lignin in addition to acetyl groups. This enables more effective enzymatic hydrolysis compared to what had originally been reflected for this step when intended to primarily remove acetate under earlier configurations employing deacetylation and dilute acid (DDA) pretreatment in prior NREL TEA models $[9,10,40]$. The move from DDA to DMR pretreatment in this update is driven by two factors. First, the ability to use a low-pressure pretreatment operation avoids logistical and operational challenges that may be incurred at commercial scale for feeding biomass across a high-pressure envelope, while avoiding the use of acid, resulting in cleaner sugars with less inhibitors for improved hydrolysis and fermentation yields [41]. Second, the inclusion of lignin upgrading to coproducts necessitates DMR as DDA pretreatment renders the lignin generally unconvertible through downstream deconstruction/conversion operations [42, 43]. 
Following alkaline extraction, DMR employs the use of a two-stage mechanical processing operation, namely disc refining followed by a secondary roller mill as shown in see Figure 3 . Rather than chemically hydrolyzing fractions of the biomass (primarily hemicellulose) in the case of DDA pretreatment, these mechanical steps delaminate and defibrillate the biomass structure, thereby increasing the surface area of the biomass for improved cellulose and hemicellulose access to enzymes [41]. Additionally, the DMR process enables higher sugar concentrations than DDA after downstream enzymatic hydrolysis at equivalent TS loading levels, given lower levels of other components such as salts and degradation products (this can be seen in NREL's recently-updated sugar model, available publicly from https://www.nrel.gov/extranet/biorefinery/aspen-models/).

In the present envisioned design, a continuous counter-current extraction unit is employed that feeds biomass and a caustic solution at opposite ends of a screw conveyor, with the resulting liquor phase routed to the lignin upgrading train and the solids phase sent on to mechanical refining and then downstream enzymatic hydrolysis.

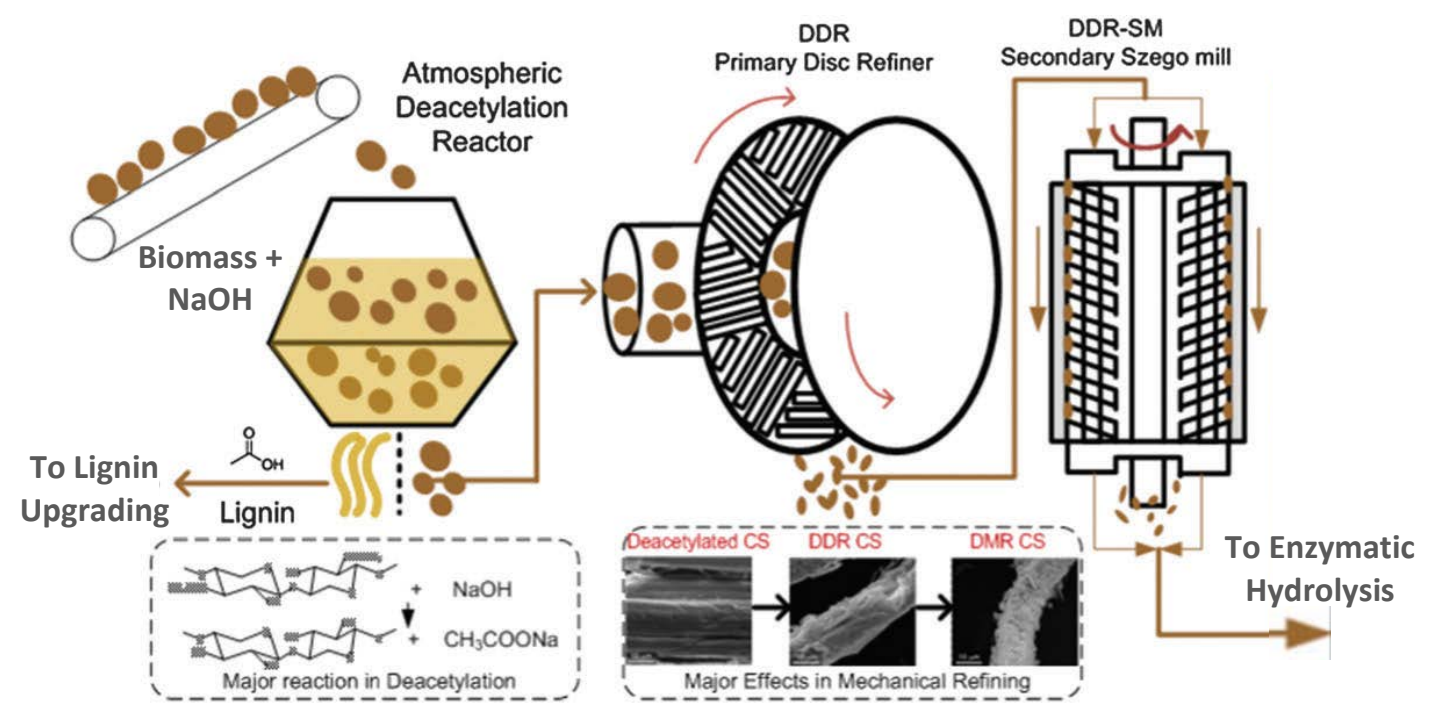

Figure 3. Schematic diagram of key DMR process operations (adopted from [41]). In this example diagram, the deacetylation/dilute alkali step is represented as a batch reactor, but is modeled in the present work as a counter-current alkaline extraction unit with internal recycle of the black liquor.

\subsubsection{Design Basis}

The deacetylation/alkaline extraction step is modified in several ways relative to the design described in prior cases [10]. First, the severity of the operation is increased in terms of caustic loading (70 $\mathrm{mg} / \mathrm{g}$ dry biomass feed versus $17 \mathrm{mg} / \mathrm{g}$ used previously for DDA pretreatment), temperature $\left(92^{\circ} \mathrm{C}\right.$ versus $80^{\circ} \mathrm{C}$ ), and residence time (90 versus 60 minutes) as required to support sufficient acetate/lignin removal for downstream efficacy of mechanical refining and enzymatic hydrolysis. Second, the configuration is modified from a batch to a continuous vessel design. The continuous design employs a counter-current extraction through an inclined screw conveyor, where the biomass is conveyed up the screw flights against a caustic solution flowing down the reactor. As biomass exits the top of the unit, a screw press squeezes liquor out of the solids material, which is recycled back to the extractor, leaving biomass at a targeted $30 \%$ total solids content to be routed to mechanical refining. The caustic exits with the black liquor, which then is re-utilized downstream in base catalyzed deconstruction of the DMR liquor combined with residual downstream lignin solids. 
This continuous counter-current operation is based on the concept of weak black liquor recycling across batch deacetylation cycles as published in Chen et al. [44]. In that cited study, proof-of-concept experiments found the ability to recycle the caustic solution in the weak black liquor across a number of batch cycles, each time increasing the strength of the black liquor and thus the concentration of acetate and solubilized lignin species (as well as other soluble solids) while generally holding carbohydrate losses constant into the liquor phase. This would prove beneficial as the single-batch deacetylation approach would yield a black liquor product too dilute for economical conversion through the lignin bioconversion step, but which cannot be concentrated either without re-precipitating the solubilized lignin components. With recycling of the liquor across multiple passes over the biomass, this both saves on makeup water demands and accordingly reduces the water content of the final black liquor product relative to other solubilized components (further discussion on this concept and its logistical considerations may be found in Chen et al. [44]). At commercial scale for processing a continuous biomass feed to the facility, the present design extrapolates the reverse-sequential batch concept investigated experimentally to employ a continuous counter-current solid-liquid extraction unit with squeezed liquor from the outlet solids recycled back through the extractor. Design and cost considerations for this unit are discussed below.

Based on preliminary NREL experimental data at the above-stated conditions, the concentrated black liquor product is projected to comprise $100 \%$ of the acetate, extractives, and protein components originally present in the delivered biomass, as well as $50 \%$ of the sucrose, $66 \%$ of the ash, and (importantly) $47 \%$ of the lignin. Of the total solubilized lignin, roughly $15 \%$ is estimated to be monomers (primarily p-coumaric, ferulic, and vanillic acids) and the remainder remains in oligomeric form, subsequently routed along with downstream residual solid lignin through base-catalyzed deconstruction under more severe conditions for further conversion to monomers (discussed later). Additionally, this step incurs losses of carbohydrates (which are targeted to be somewhat mitigated through the counter-current liquor recycling design), set at targets of $2 \%$ glucan, $10 \%$ xylan, and $30 \%$ arabinan solubilizations into the liquor product. Although these losses from the fuel train are higher than prior targets with milder deacetylation conditions employed for DDA pretreatment [10], these solubilized carbohydrates are still utilized for conversion to biomass and muconic acid through the lignin coproduct train downstream, and so are not lost in valorization potential altogether. The water content of the concentrated black liquor is estimated at roughly $66 \mathrm{wt} \%$, at a $\mathrm{pH}$ of 8-9. The projections asserted here for this operation carry a degree of risk as this has not yet been tested experimentally at NREL in this continuous configuration, and thus represents an important area for future study to validate or refine the present assumptions.

The extracted solids material is dewatered through a screw discharger at the end of the counter-current alkaline extraction unit and routed to mechanical refining. The mechanical refining operations consist of a primary disc refiner and a secondary step such as a roller mill or low-consistency disc refining (either option is anticipated to provide comparable performance), which together require a power demand of $200 \mathrm{KWh}$ per dry tonne of processed solids based on previous vendor guidance. This power demand was previously found to represent an optimum between mechanical disruption of the biomass versus reasonable biorefinery power costs [42], given that this is a power-intensive step (although it saves on heat/steam demands, which are required for DDA pretreatment). The resulting particle size exiting DMR is typically 200-300 microns on average. All pertinent parameters for the DMR pretreatment step are summarized in Table 2. 
Table 2. DMR Pretreatment Conditions Applied in this Design a

\begin{tabular}{lc}
\hline Parameter & Current design \\
\hline Deacetylation (mild alkaline extraction) & $92{ }^{\circ} \mathrm{C}$ \\
Temperature & $90 \mathrm{~min}$ \\
Residence time & $70 \mathrm{mg} / \mathrm{g} \mathrm{dry} \mathrm{biomass}$ \\
Total caustic (NaOH) loading & $2 \%$ \\
Net solubilized glucan to liquor (wt $\%)$ & $10 \%$ \\
Net solubilized xylan to liquor (wt $\%)$ & $30 \%$ \\
Net solubilized arabinan to liquor (wt $\%)$ & $66 \%$ \\
Ash removal (wt\%) & $100 \%(10 \mathrm{~g} / \mathrm{L})$ \\
Solubilized acetate (wt $\%)$ & $100 \%(79 \mathrm{~g} / \mathrm{L})$ \\
Solubilized extractives (wt $\%)$ & $47 \%(40 \mathrm{~g} / \mathrm{L})$ \\
Solubilized lignin (wt $\%)$ & $10 \%$ \\
P-coumaric acid (wt\% of solubilized lignin) & $4 \%$ \\
Ferulic acid (wt\% of solubilized lignin) & $1 \%$ \\
Vanillic acid (wt $\%$ of solubilized lignin) & $85 \%$ \\
Lignin oligomers (wt\% of solubilized lignin) & \\
Mechanical Refining & $30 \%$ \\
Solids loading (wt $\%)$ & 200 \\
Power demand (KWh/dry tonne processed biomass) & \\
\hline
\end{tabular}

${ }^{a}$ DMR parameters are generally based on NREL experimental data observed to date on DMR processing with batch deacetylation, but extrapolated to higher targeted black liquor concentrations and marginally lower carbohydrate losses targeted for counter-current deacetylation/alkaline extraction.

A small flash tank is also maintained following the DMR process, but flashes a much smaller amount of steam than in the DDA design. From there, the slurry is routed to enzymatic hydrolysis after being diluted with water as appropriate for the given hydrolysis design considered (discussed below).

\subsubsection{Cost Estimation}

The counter current deacetylation unit was costed based on a Braunschweigische Maschinenbauanstalt AG sugar beet cossette extraction unit $[45,46]$. This unit is designed to withdraw liquid from the solid cossettes via a double conical screen and is sized based on the biomass solid flow rate. For the mechanical refining equipment, both the disc refiner and roller mill (here, based on a Szego mill [https://www.szegomill.com/]) were based on vendor cost quotations provided when NREL began investigating DMR pretreatment, requiring 8 and 11 units respectively at a purchase cost of roughly $\$ 2.5 \mathrm{MM}$ and \$580,000 per unit (2013\$). In all, Area 200 contributes roughly \$1.42/GGE and $\$ 1.48 /$ GGE to the MFSP for the acids and BDO cases, respectively, including deacetylation, mechanical refining, and conditioning. About $31 \%$ of this is attributed to capital cost with the remainder attributed to caustic and power.

\subsection{Area 300: Enzymatic Hydrolysis and Hydrolysate Conditioning}

\subsubsection{Overview}

In this area, pretreated biomass is sent through enzymatic hydrolysis to convert polysaccharides to monomeric sugars using cellulase and hemicellulase enzymes, followed by hydrolysate conditioning in the case of the acids pathway. For the BDO pathway, enzymatic hydrolysis employs NREL's historical design assumptions including a 24-hour continuous vertical vessel followed by standard batch saccharification reactors. The whole-slurry hydrolysate (including solids) is then cooled and subjected to batch fermentation in the same tanks, reflecting a similar strategy as prior ethanol 
pathway models [2]. However, for organization purposes, the fermentation portion of the batch time and associated tankage volume is allocated to A500 while the hydrolysis portion is allocated to A300.

For the acids pathway, a more novel continuous enzymatic hydrolysis (CEH) operation is employed utilizing a series of saccharification tanks in sequence, with each connected to a microfiltration membrane through a pump-around loop to remove sugars as they are produced, thereby reducing feedback inhibition and improving hydrolysis kinetics. The remaining solids are routed to the next hydrolysis vessel to repeat the process. Enzymes initially lost through the microfilters are subsequently recovered through an ultrafiltration step on the combined sugar product stream. The $\mathrm{CEH}$ process is targeted to achieve upwards of $96 \%$ hydrolysis of carbohydrates to monomeric sugars, but requires more dilute operating conditions (generally below $10 \%$ total solids), thus the clarified hydrolysate must be re-concentrated in a vacuum mechanical vapor recompression (MVR) evaporator. The clarified, concentrated hydrolysate is routed to downstream fermentation. Both A300 process schematics are depicted in Figure 4.

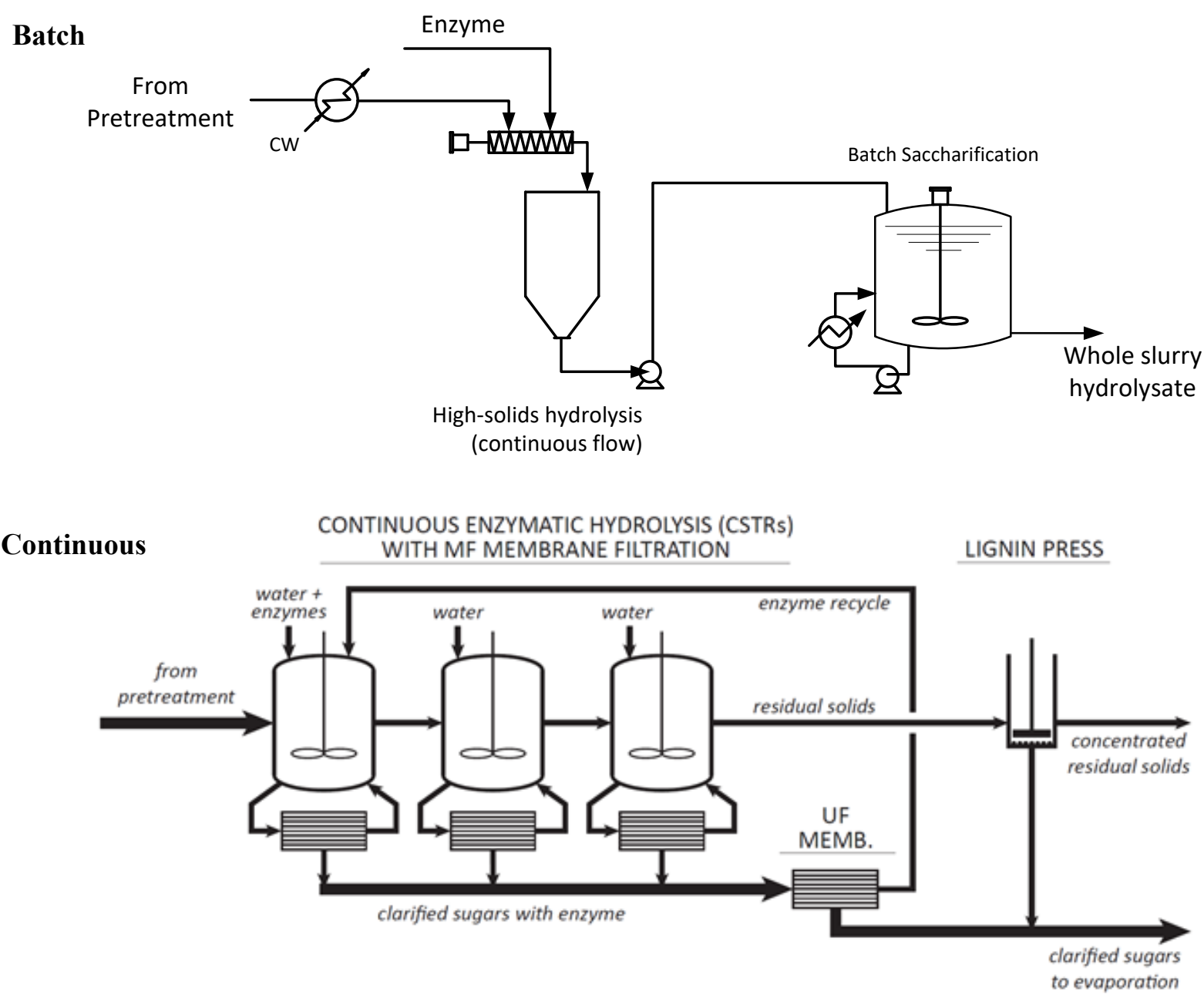

Figure 4. Process schematic diagrams for batch and continuous enzymatic hydrolysis/hydrolysate conditioning operations (BDO and acids pathways respectively) 


\subsubsection{Design Basis}

\section{BDO Pathway: Batch Enzymatic Hydrolysis}

In the BDO case, the process and design basis for enzymatic hydrolysis is the same as described in the 2011 ethanol report and 2013 biological hydrocarbon report, thus will only be briefly summarized here. In short, enzymatic hydrolysis is initiated in a continuous, high-solids vertical tower reactor with the slurry flowing down the reactor by gravity; this first step is required as the feed material at $25 \%$ solids (or more) is not pumpable until the cellulose has been partially hydrolyzed. After mixing in the cellulase enzyme, the total solids loading into the continuous column reactor is $25 \mathrm{wt} \%$ and the temperature is $50^{\circ} \mathrm{C}\left(122^{\circ} \mathrm{F}\right)$. The residence time in the continuous reactor is 24 hours. After this point, the slurry is pumpable and is batched to one of eight $1 \mathrm{MM}$-gal vessels (950,000 gal working volume), where enzymatic hydrolysis continues for another 96 hours ( 5 days total hydrolysis time). The batch reactors are agitated and temperature controlled at $50^{\circ} \mathrm{C}$ using a pump-around loop with cooling water heat exchange. This represents a slight increase in hydrolysis temperature relative to prior design cases $\left(48^{\circ} \mathrm{C}\right)$, based on experimental conditions more recently employed. When hydrolysis is completed, the hydrolysate is cooled and fermentation is initiated in the same vessels, discussed in Section 3.5. This is consistent with the sequential hydrolysis and fermentation (SHF) approach taken in the 2011 ethanol design case, but more recent review feedback suggested that this may pose a higher risk of contamination as the hydrolysate is cooled in the same tank for initiating fermentation. However, the alternative to send the hydrolysate to a heat exchanger and then a separate tank for fermentation would carry a minimal cost difference as the hydrolysis and fermentation vessels are the same and are sized/costed based on summative batch times for hydrolysis plus fermentation.

The amount of enzyme used (the enzyme loading) is still reported here based on the amount of cellulose present in the hydrolysate, even though the enzyme now must include both cellulase and hemicellulase activity given that hemicellulose was not substantially hydrolyzed upstream in DMR pretreatment as is achieved in DDA pretreatment. Total enzyme loading is targeted at $10 \mathrm{mg}$ enzyme protein/g cellulose with a target of $90 \%$ conversion of cellulose to glucose and xylan to xylose, as well as $85 \%$ arabinan to arabinose. NREL's latest 2017 state-of-technology performance efforts demonstrated $78 \%$ glucan conversion and $85 \%$ xylan conversion to monomeric sugars at a $12 \mathrm{mg} / \mathrm{g}$ total enzyme loading on DMR substrates. Thus, the targets proposed here to be achieved by the year 2030 are not unreasonable projections given ongoing advancements in enzyme development. Directionally less enzyme is also needed than prior designs due to the reduction in active lignin deactivation sites given high upstream lignin removal. However, an additional risk is posed in continuing shifts to new feedstock blends rather than a single feedstock (corn stover), which may prove more recalcitrant to enzymatic hydrolysis. The target design conditions for enzymatic hydrolysis are summarized in Table 3.

Table 3. Standard Enzymatic Hydrolysis Conditions

\begin{tabular}{ll}
\hline Temperature & $50^{\circ} \mathrm{C}\left(122^{\circ} \mathrm{F}\right)$ \\
Initial solids loading & $25 \mathrm{wt} \%$ total solids \\
Residence time & 5.0 days total $(96 \mathrm{~h})$ \\
Number and size of continuous vessels & $6 @ 950 \mathrm{~m}^{3}(250,000 \mathrm{gal})$ each \\
Number and size of batch vessels & $8 @ 3,600 \mathrm{~m}^{3}(950,000 \mathrm{gal})$ each \\
Total enzyme (cellulase + hemicellulase) loading & $10 \mathrm{mg}$ protein $/ \mathrm{g}$ cellulose \\
\hline
\end{tabular}




\section{Acids Pathway: Continuous Enzymatic Hydrolysis and Concentration}

In the acids case, because acids fermentation includes circulation of the fermentor broth through a pertractive membrane for recovery of the acids, insoluble solids must first be removed to avoid clogging the membrane. This could be accomplished using a similar batch enzymatic hydrolysis step as for the BDO pathway, followed by a vacuum belt filter press with addition of a flocculant. However, this operation incurs significant costs for both the filter press capital expenses as well as the cost of the flocculant; filtration is also more challenging for DMR hydrolysate, requiring high flocculant loadings (at least $20 \mathrm{~g} / \mathrm{kg}$ insoluble solids) at lower membrane permeances (at most $15 \mathrm{~kg}$ insoluble solids $/ \mathrm{m}^{2}$-h) to achieve 95\% sugar recoveries (as may be found in NREL's sugar model scenarios available from https://www.nrel.gov/extranet/biorefinery/aspen-models/).

Alternatively, recent NREL research has begun investigating the more novel continuous enzymatic hydrolysis approach wherein hydrolysis is initiated in a continuous stirred tank reactor (CSTR) vessel connected through a pump-around loop to a microfilter, which continuously removes sugars as they're produced while retaining the unhydrolyzed solids. This improves hydrolysis kinetics by reducing sugar feedback inhibition, allowing for lower hydrolysis volumes, higher sugar yields, and/or lower enzyme loadings based on NREL proof-of-concept experimental work at bench scale (3 L reactor volumes operated over 80 hours) [47]. The residual solids from one CSTR step are routed to a subsequent CSTR with the same setup, and this process is repeated several times (in this design, using three CEH reactors in series). Enzyme retention through the microfiltration units is not perfect, and "leaked" enzyme is captured and recycled through a subsequent ultrafilter located on the combined microfilter permeate streams. Prior TEA modeling for this concept demonstrated the potential for significant MFSP cost savings, on the order of $\$ 1.50 / \mathrm{GGE}$ relative to a benchmark case employing batch enzymatic hydrolysis (EH) and vacuum belt filtration [12], albeit the reference case was based on older enzyme performance levels reflective of NREL's 2012 ethanol demonstration runs [40]. Relative to newer enzymes available currently (or moving into future years), at $90 \%$ projected sugar yields, the magnitude of MFSP savings would not be as large for CEH. However, with the projection of such yields for batch $\mathrm{EH}$ in the future, $\mathrm{CEH}$ is envisioned to improve on this further by achieving at least $96 \%$ conversions to monomeric sugars while maintaining the same $10 \mathrm{mg} / \mathrm{g}$ targeted enzyme loading.

It bears noting that this $\mathrm{CEH}$ concept carries a risk that is not yet well-understood, regarding the ability of all enzyme proteins to maintain a consistent composition over numerous recycles. Particularly given the use of both cellulase and hemicellulase enzyme cocktails in this design, different enzymes may have different levels of resistance to shear, oxidation, and denaturation across the multiple filtration and recycle steps, and different enzyme proteins may not be retained equally which may lead to a compositional imbalance in the cocktail mixture over time. This has not yet been investigated experimentally at NREL over longer-term trials and remains an area of future study, although it is known that different components have different binding characteristics and that binding affinities can be modified through protein engineering (an activity that NREL hopes to investigate in the future to reduce non-productive binding to lignin-rich residues).

While CEH can achieve improved hydrolysis yields, it also is limited to lower solids loadings because each stage is maintained at a consistent IS level after dilution with water (i.e., IS does not decrease over the course of the process as it does in batch EH). The operation is targeted at $7.5 \%$ IS loading (roughly $7.6 \%$ TS loading) as a maximum level constrained by CSTR agitation ability and pumpability through the membrane pump-around loops. Current experimental work has so far been done closer to $5 \%$ IS levels, but $7.5 \%$ is viewed by NREL researchers as a plausible future target. This more dilute 
operation translates to high water content in the solids outlet stream from the final stage, roughly $87 \%$ moisture content, which is too high to be sent directly to the boiler. Thus, the original lignin press unit, as utilized in NREL's 2011 ethanol case [2] for separating lignin from the ethanol beer stillage, is reincorporated at this point to reduce water content of the solids stream to $35 \%$, which also carries an additional benefit of recovering $95 \%$ of the sugars initially lost with the solids (thus translating to an overall recovery of $99 \%$ of all produced sugars across $\mathrm{CEH}$ ). Over $90 \%$ of the water is also removed from the solids with the clarified sugars, with a minimal amount of water evaporated from air drying.

While the inlet and product water content of the solids across the lignin press is similar to the original conditions for that unit in the ethanol process, because the solids here are from DMR pretreatment, filterability across the lignin press will be more challenging, and may require the assistance of a flocculant (as is required for the vacuum filter press when processing the main hydrolysate stream). This is not yet well-understood but will be further considered in the future. The target design conditions for $\mathrm{CEH}$ are summarized in Table 4.

Table 4. Continuous Enzymatic Hydrolysis Conditions

\begin{tabular}{ll}
\hline Temperature & $50^{\circ} \mathrm{C}\left(122^{\circ} \mathrm{F}\right)$ \\
Steady-state solids loading & $7.6 \mathrm{wt} \%$ total solids $(7.5 \%$ insoluble $/ 0.1 \%$ soluble $)$ \\
Number of CSTR stages & 3 \\
Stage residence time & $30 \mathrm{hr}-53 \mathrm{hr}-71 \mathrm{hr}$ \\
Total enzyme (cellulase + hemicellulase) loading & $10 \mathrm{mg} \mathrm{protein} / \mathrm{g}$ cellulose \\
Microfiltration permeance & $100 \mathrm{~kg} / \mathrm{m}^{2} / \mathrm{hr}$ \\
Total microfiltration area & $95,783 \mathrm{ft}^{2}\left(8,898 \mathrm{~m}^{2}\right)$ \\
Ultrafiltration permeance & $67 \mathrm{~kg} / \mathrm{m}^{2} / \mathrm{hr}^{2}$ \\
Total ultrafiltration area & $114,940 \mathrm{ft}^{2}\left(10,678 \mathrm{~m}^{2}\right)$ \\
Overall recovery of produced sugars & $99 \%$ \\
\hline
\end{tabular}

The conversions taking place during both standard and continuous enzymatic hydrolysis are listed in Table 5. As noted above, because enzymatic hydrolysis must convert both cellulose and hemicellulose components to monomeric sugars given the use of DMR pretreatment, these sugar yields implicitly indicate a combination of both cellulase and hemicellulase activity for the total enzyme loading specified above.

Table 5. Standard and Continuous Enzymatic Hydrolysis Reactions and Assumed Conversions

\begin{tabular}{lccc}
\hline Reaction & Reactant & $\begin{array}{c}\text { \% Conversion } \\
\text { (Standard EH) }\end{array}$ & $\begin{array}{c}\text { \% Conversion } \\
\text { (Continuous EH) }\end{array}$ \\
\hline (Glucan) $\mathrm{n}+\mathrm{n} \mathrm{H}_{2} \mathrm{O} \rightarrow \mathrm{n}$ Glucose & Glucan & $90 \%$ & $96.3 \%$ \\
(Glucan) $\mathrm{n}+\mathrm{n} \mathrm{H}_{2} \mathrm{O} \rightarrow \mathrm{n}$ Cellobiose & Glucan & $1.2 \%$ & $0 \%$ \\
$(\text { Xylan })_{\mathrm{n}}+\mathrm{n} \mathrm{H}_{2} \mathrm{O} \rightarrow \mathrm{n}$ Xylose & Xylan & $90 \%$ & $98.8 \%$ \\
$(\text { Arabinan })_{\mathrm{n}}+\mathrm{n} \mathrm{H}_{2} \mathrm{O} \rightarrow \mathrm{n}$ Arabinose & Arabinan & $85 \%$ & $98.8 \%$ \\
\hline
\end{tabular}

The clarified filtrate exiting microfiltration and ultrafiltration from $\mathrm{CEH}$ is also fairly dilute at $5.5 \%$ sugars, and is thus pumped to hydrolysate concentration, consisting of a four-stage MVR evaporation system, which produces a concentrated sugar stream of $49 \%$ (monomeric) sugar, $50 \%$ water, and $1 \%$ other dissolved solids for downstream acid fed-batch fermentation. The $50 \%$ total solids specification is set as an estimated balance between evaporation cost, syrup viscosity, and downstream fermentation sizing/operation, but further room exists for optimization on this parameter in the future. Also by including hydrolysate concentration, this provides a more microbially stable stream for fermentation. 
The evaporator design and cost basis is consistent with prior design reports [10]. To avoid the possibility of sugar degradation at high temperatures [48], the evaporators are assumed to be operated under slight vacuum to keep the maximum temperature below $90^{\circ} \mathrm{C}\left(194^{\circ} \mathrm{F}\right)$. The vapor exiting the evaporation system is nearly all water (approximately $99.9 \%$ purity) and can be recycled directly to the process water manifold after being condensed by water cooling. The vendor-provided evaporation system design specifications are summarized in Table 6.

Table 6. CEH Filtrate Evaporator Specifications

\begin{tabular}{ll}
\hline Feed sugar concentration & $5.5 \mathrm{wt} \%$ \\
Product sugar concentration & $49 \mathrm{wt} \%$ (50\% water, 1\% other solubles) \\
Maximum operating temperature & $87^{\circ} \mathrm{C}$ \\
Evaporator technology & $\mathrm{MVR}$ \\
Number of effects & 4 \\
Electricity usage & $14,454 \mathrm{KW}(19,383 \mathrm{hp})$ \\
Steam usage (low-pressure steam) & $1,550 \mathrm{~kg} / \mathrm{hr}(0.8 \mathrm{MMkcal} / \mathrm{hr})$ \\
\hline
\end{tabular}

\subsubsection{Cost Estimation}

For the BDO case utilizing the standard enzymatic hydrolysis operations, the design and cost basis assumptions for all hydrolysis equipment were left unchanged from prior design cases [2, 10], namely empty towers for the continuous hydrolysis reactor based on a vendor quotation for flat-bottomed plug-flow reactors with a 10:1 height to diameter ratio, as well as $1 \mathrm{MM}$-gal batch hydrolysis reactors and agitators. The material of construction for enzymatic hydrolysis equipment is 304SS. As noted above, after batch hydrolysis is complete, the hydrolysate is cooled and BDO fermentation is initiated in the same $1 \mathrm{MM}$-gal vessels; however, for cost tracking purposes, the portion of the batch time (4 days) and associated tankage volume (5.7 MM gal) attributed to hydrolysis is assigned to Area 300 , with the remainder for BDO fermentation (1.5 days and 2.1 MM gal) assigned to Area 500.

For the acids case utilizing CEH, the CSTR vessels are costed consistently with the standard batch hydrolysis reactors noted above, using multiple $1 \mathrm{MM}$-gal vessels for each stage and thus costing the reactors based on total volume with a linear scaling factor. All membrane costs are based on subcontractor guidance for ultrafiltration membranes, with costs scaled linearly based on membrane area (calculated from the given permeance targets above). The original quoted cost basis was $\$ 2.05$ MM (2011\$) for a membrane area originally sized at 53,820 $\mathrm{ft}^{2}$. This basis is applied for both the microfilter and ultrafilter membranes here, thus may err on the conservative side for the larger pore size microfiltration units, but allows for a safety factor in the costing for this more preliminary $\mathrm{CEH}$ concept. A provision was also included for an additional spare membrane unit in the event of membrane fouling/plugging or other required maintenance and membrane lifetime was assumed to be two years. The sugar concentration (MVR evaporation) equipment is also based on vendor-provided design and cost estimates, consistent with information provided in the 2013 design report [10].

\subsection{Area 400: Enzyme Production}

\subsubsection{Overview}

This process area produces cellulase and hemicellulase enzymes used in Area 300 to hydrolyze cellulose into glucose and xylan/arabinan into xylose/arabinose respectively. Cellulase is produced industrially using (among other microorganisms) T. reesei, a filamentous fungus that secretes high levels of cellulase enzymes when grown aerobically in the presence of cellulose or other cellulase 
inducers. The present analysis maintains consistency with the fundamental assumptions for enzyme production and cost estimation as detailed in the 2011 ethanol report and 2013 biological hydrocarbon report, most importantly, the use of on-site enzyme production rather than a purchased-enzyme model. As stipulated in prior reports, we again note that by including an on-site enzyme production section, NREL and DOE are not making a judgment about whether or not the cellulosic biofuel industry should align to this mode of enzyme distribution. Rather, the model on-site enzyme section is intended to improve transparency in determining the true cost of cellulase enzymes for large-scale cellulosic biofuel production.

Consistent with earlier design cases, the present design considers submerged aerobic cultivation ("aerobic fermentation") of a T. reesei-like fungus on a feedstock of glucose and fresh water. While hydrolysate could alternatively be used directly to cultivate the enzyme, the use of glucose likely enables higher enzyme titers and thus lower capital costs and utility demands. We have assumed a media preparation step where a small fraction of glucose is converted to sophorose, a powerful inducer of cellulase, using a small amount of the cellulase enzyme itself. When grown on this substrate, $T$. reesei has been shown to productively secrete cellulase [49]. It is noted that one difference in the present work is that both cellulase and hemicellulase enzymes are required in the enzymatic hydrolysis step, but the same overall enzyme production process framework is maintained, assuming the costs of producing a quantity of enzyme protein are similar whether for cellulase or hemicellulase. This may require two production host strains given practical limitations to the number of enzymes a single strain can express, but in that case if both strains could be co-cultivated in the same shared bioreactor configuration (i.e. did not require two parallel process trains), the overall TEA cost implications would be minimal. This assumption may be revisited in future work if further validation or guidance from industry is received. The whole broth product is transferred to the hydrolysis tanks without an enzyme isolation step. Figure 5 is a simplified flow diagram of the enzyme production section.

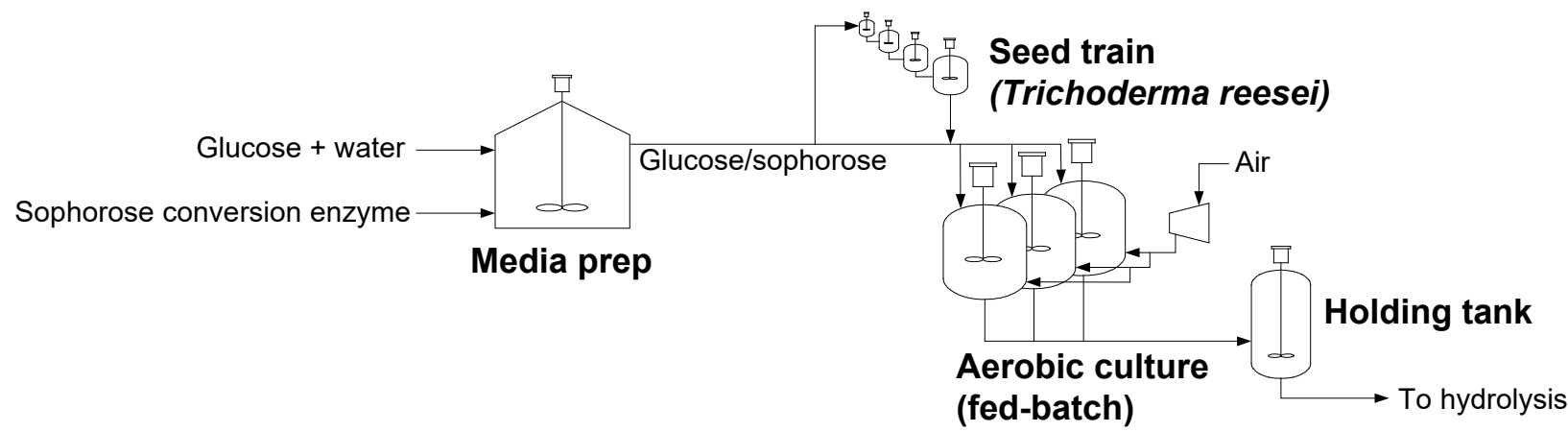

Figure 5. Simplified flow diagram of the enzyme production process

\subsubsection{Design Basis}

The design for the enzyme production operations is described in detail in the 2011 ethanol report and will not be repeated in such detail here. In summary, the design for the system was based in part on the claims in Example 5 of U.S. Patent 4,762,788 [50,51] in combination with a number of reasonable assumptions to develop a rudimentary process as documented in the 2011 ethanol report. The key assumptions used in the current design are summarized in Table 7. 
Table 7. Area 400 Guiding Design Basis Assumptions

\begin{tabular}{ll}
\hline Parameter & Assumption \\
\hline Protein loading to enzymatic hydrolysis & $10 \mathrm{mg}$ protein/g cellulose \\
Reactor size & $300,000 \mathrm{~L} \mathrm{@} \mathrm{80 \%} \mathrm{final} \mathrm{working} \mathrm{volume}$ \\
Enzyme titer at harvest & $50 \mathrm{~g} / \mathrm{L}$ \\
Mass yield of enzyme from glucose & $0.24 \mathrm{~kg}$ enzyme $/ \mathrm{kg}$ glucose \\
Enzyme production cycle time & $120 \mathrm{~h}$ online, $48 \mathrm{~h}$ offline, $168 \mathrm{~h}$ total \\
\hline
\end{tabular}

The targeted total cellulase and hemicellulase loading to enzymatic hydrolysis is set in the present pathway model to $10 \mathrm{mg}$ of enzyme protein per $\mathrm{g}$ of cellulose as also assumed in the 2013 biological conversion report, down from $20 \mathrm{mg} / \mathrm{g}$ in the 2011 ethanol report, as described in Section 3.3. Here, "protein" refers to the total amount of high molecular weight protein in the enzyme broth as determined by assay; not all of this protein is active cellulase/hemicellulase. The total protein demand was thus calculated to be $260 \mathrm{~kg} / \mathrm{h}(570 \mathrm{lb} / \mathrm{h})$. An additional $10 \%$ is produced to account for the slipstream provided to the media preparation tank to make the glucose/sophorose mixture.

The size of the cellulase production vessels was set at $300 \mathrm{~m}^{3}(80,000 \mathrm{gal})$ with a height-to-diameter ratio of 2 . Fermentation is assumed to be a fed-batch process starting at $50 \%$ working volume and ending at $80 \%$. Over one week, each bioreactor will see a 24 -hour cell growth period, a 96-hour protein production period, and a 48-hour offline period for draining, cleaning, and refilling. With a 1week total cycle time and the production parameters listed in Table 7, one bioreactor is capable of producing $12,000 \mathrm{~kg}$ of protein in a week, or $71.4 \mathrm{~kg} / \mathrm{h}(157 \mathrm{lb} / \mathrm{h})$. The equivalent enzyme volumetric productivity is $0.30 \mathrm{~g}$ protein/L-h. Five production reactors were therefore required to deliver the 260 $\mathrm{kg} / \mathrm{h}$ of protein needed for enzymatic hydrolysis. The reactors are loaded initially with the glucose/sophorose carbon source and nutrients, including corn steep liquor (CSL), ammonia, and sulfur dioxide $\left(\mathrm{SO}_{2}\right)$. After the initial cell growth period, additional substrate is added to maintain protein production. The bioreactors are sparged with compressed and cooled air and corn oil is added as antifoam. The reactors are temperature-controlled by chilled water flowing through internal coils.

Aeration and agitation requirements for the production bioreactors are functions of oxygen transfer rate (OTR) and oxygen uptake rate (OUR), which have been discussed in detail in the 2011 ethanol report and again in the 2013 report. Thus, the details and governing principles for these metrics will not be repeated again here. In the modeled bioreactors, the reaction stoichiometry balances the reactions of substrate, oxygen, ammonia, and $\mathrm{SO}_{2}$ to cell mass and enzyme (plus $\mathrm{CO}_{2}$ and water) using an elemental composition for commercial enzyme provided by Novozymes [51]. The composition of cell mass was taken as the average of a generic cell mass composition [52] and the enzyme composition, with the assumption that cell mass includes some unreleased protein.

Enzyme protein: $\mathrm{CH}_{1.59} \mathrm{~N}_{0.24} \mathrm{O}_{0.42} \mathrm{~S}_{0.01}$

\section{T. reesei cell mass: $\mathrm{CH}_{1.645} \mathrm{O}_{0.445} \mathrm{~N}_{0.205} \mathrm{~S}_{0.005}$}

In the production bioreactors, it is assumed that $90 \%$ of the carbon source is converted via the protein reaction and $10 \%$ is converted via the cell mass reaction. In the seed reactors, $85 \%$ of the carbon is converted via the cell mass reaction and $5 \%$ via the protein reaction, with $10 \%$ unreacted. This represents an overall molar selectivity of glucose to $31 \%$ protein, $4 \%$ cell mass, and $65 \% \mathrm{CO}_{2}$, 
yielding $0.24 \mathrm{~kg}$ enzyme protein $/ \mathrm{kg}$ glucose. The final specifications for the enzyme production reactors are shown in Table 8.

\section{Table 8. Specifications of the Enzyme Production Bioreactors}

\begin{tabular}{ll}
\hline Total volume & $300 \mathrm{~m}^{3} / 80,000 \mathrm{gal}$ \\
Maximum working volume & $80 \%$ \\
Height-to-diameter ratio & 2 \\
Height & $11.5 \mathrm{~m}$ \\
Diameter & $5.75 \mathrm{~m}$ \\
Operating pressure & $1 \mathrm{~atm}$ \\
Operating temperature & $28^{\circ} \mathrm{C}\left(82^{\circ} \mathrm{F}\right)$ \\
Material & $316 \mathrm{SS}$ \\
Agitator & $800 \mathrm{hp}$ \\
Total electricity demand per kg protein & $9 \mathrm{KWh} / \mathrm{kg}$ \\
(air compressors, agitators, chillers, pumps) & \\
\hline
\end{tabular}

Four trains of three seed fermentors provide inoculum to the main enzyme production bioreactors. Each vessel in the seed trains is run batchwise on the same substrate as the production vessels. It is possible that by running in a semi-continuous fill-and-draw mode, the seed tankage volume and complexity could potentially be minimized to reduce the number of seed stages, but this has not been evaluated through dynamic process modeling and thus the standard three-stage batch operation is maintained. Air is also sparged through each of the seed vessels, which are cooled with chilled water. The seed bioreactors are each sized at $10 \%$ of the next stage volume, i.e., $0.3 \mathrm{~m}^{3}, 3 \mathrm{~m}^{3}$, and $30 \mathrm{~m}^{3}$. The aeration demand is assumed to be $10 \%$ of the production aeration rate. Four trains were chosen because each production fermentor has a total cycle time of 7 days; each seed fermentor should have a cycle time of 2 days (including cleaning and sterilization) to get through the cell growth phase only.

Like the oxygen uptake rate, the glucose demand is also computed stoichiometrically from the required protein production rate. Ammonia and $\mathrm{SO}_{2}$ are fed to the reactors stoichiometrically and CSL, trace nutrients, and antifoam (corn oil) are added to the substrate based on flow rate. The required nutrient concentrations are based on Schell et al. [53] and remain the same as presented in prior design reports. Glucose, the carbon source for cell mass and protein, is the most significant enzyme production expense in this model. The cost for glucose was updated to the latest five-year average price of glucose syrup, as discussed in Section 4.3. Electricity also remains a significant contributor due to the power requirements of air injection, agitation, and refrigeration. Total electricity demand for these operations is shown in Table 8.

\subsubsection{Cost Estimation}

The cost estimation for all equipment in A400 was left unchanged from the basis values provided in the 2011 ethanol report and maintained in the 2013 case, thus will not be repeated in detail here. Most equipment in this area is stainless steel. The air compressor and some of the nutrient delivery equipment items are specified as carbon steel. Quotes for the production bioreactors, internal cooling coils, production agitators and motors, skid-mounted seed fermentors, and air compressor were provided by vendors through Harris Group, which developed costs for the pumps in this area using their historical database. Not included in the enzyme production model are any costs for concentration, stabilization, or transportation of the enzyme to the plant, which would not be required in this case for on-site production. Whether for on- or off-site enzyme production, one expects to have to pay 
licensing fees for the cellulase production microorganism, but that cost is not included as any amount would be speculative without availability of such licensing costs; however, a reasonable range is considered later in the Sensitivity Analysis section. The enzyme production system is also sized strictly to provide the amount of enzyme required for the hydrolysis step (e.g., is not oversized to accommodate occasional poor-yielding runs), reflective of the $n^{\text {th }}$-plant approach taken elsewhere in the design (e.g., avoiding over-design of equipment operations).

Based on the economics of the on-site enzyme section described above, the predicted cost of enzymes to the facility is roughly $\$ 0.43 / \mathrm{GGE}$ of total fuel product in the acids case example. The resulting enzyme cost on a per-kg basis is $\$ 6.16 / \mathrm{kg}$ protein in $2016 \$$. The caveat discussed in prior design reports bears repeating, that the enzyme cost contribution modeled here is lower than one would expect for an enzyme preparation purchased from a separate, non-adjacent production facility. Transportation of the enzyme to the biorefinery facility could add a non-trivial amount to enzyme costs, even if formulation costs could be avoided. Furthermore, by lumping the enzyme production equipment in with the biorefinery, some key items are inherently shared, e.g., the land and buildings, cooling tower, and utilities infrastructure. Overhead and fixed costs, especially labor and management, would also be higher for a standalone facility. Additionally, an external enzyme production facility would probably demand a higher rate of return than the $10 \%$ IRR assumed for the biorefinery plant because it is a higher-risk and lower-volume business. Still, in the near term it is more likely that cellulosic biorefineries (whether targeting sugars, ethanol, or hydrocarbons) will purchase enzyme from an external supplier with an organization dedicated to improving enzyme performance and reducing costs.

\subsection{Area 500: Fermentation, Catalytic Conversion, and Upgrading}

\subsubsection{Overview}

Area 500 includes all core processing steps for conversion of the liberated sugars to hydrocarbon fuels/blendstocks via biological conversion to intermediate fuel precursors and catalytic upgrading of those intermediates to finished products. Both bioconversion pathways are anaerobic as introduced previously and further rationalized below.

In the BDO pathway, after batch enzymatic hydrolysis is completed, the hydrolysate is cooled and batch fermentation is initiated in the same vessels. BDO fermentation utilizes an engineered strain of Zymomonas mobilis to convert sugars to 2,3-BDO plus hydrogen as a byproduct for achieving cell redox balancing (the latter is purified from the fermentation off-gas and used downstream to help meet catalytic upgrading hydrogen demands). The fermentation is conducted on whole-slurry hydrolysate with the presence of solids, targeted here at $25 \%$ total solids. The fermentor product broth is routed to a lignin press to remove solids, and then to a polishing filter to remove particle fines and ion exchange to mitigate ionic/salt species that may be problematic for the downstream catalyst. The aqueous BDO stream is then heated at elevated pressure and routed to catalytic BDO upgrading, producing butene and minor byproducts. The butene product is distilled from water and upgraded to oligomers in the C8-C16 range, and then finally sent through a hydrotreating step to saturate the oligomers to paraffinic hydrocarbons.

In the acids pathway, the clarified/concentrated hydrolysate is routed to anaerobic fermentation using Clostridium tyrobutyricum to produce butyric acid, which is continuously removed as it is produced through a pump-around loop connected to an extractive membrane (pertraction) system. The acid 
passes through the membrane into a solvent phase, then is sent to distillation to recover and recycle the solvent. Similar to the BDO pathway, the acid fermentation step also co-produces hydrogen, which is purified from the off-gas and used to help satisfy biorefinery hydrogen demands downstream. The recovered acid is then catalytically upgraded through a ketonization step to (primarily) 4-heptanone and $\mathrm{CO}_{2}$, with the latter flashed off and routed to a scrubber to recover volatilized ketone. Next, the material is upgraded across a condensation step to an oxygenated C14 component in the presence of a solvent (subsequently distilled and recycled), and finally routed through hydrotreating to remove oxygen, leaving an isomerized $\mathrm{C} 14$ hydrocarbon for use as a diesel blendstock. The key unit operations for both pathways are shown schematically in Figure 6.

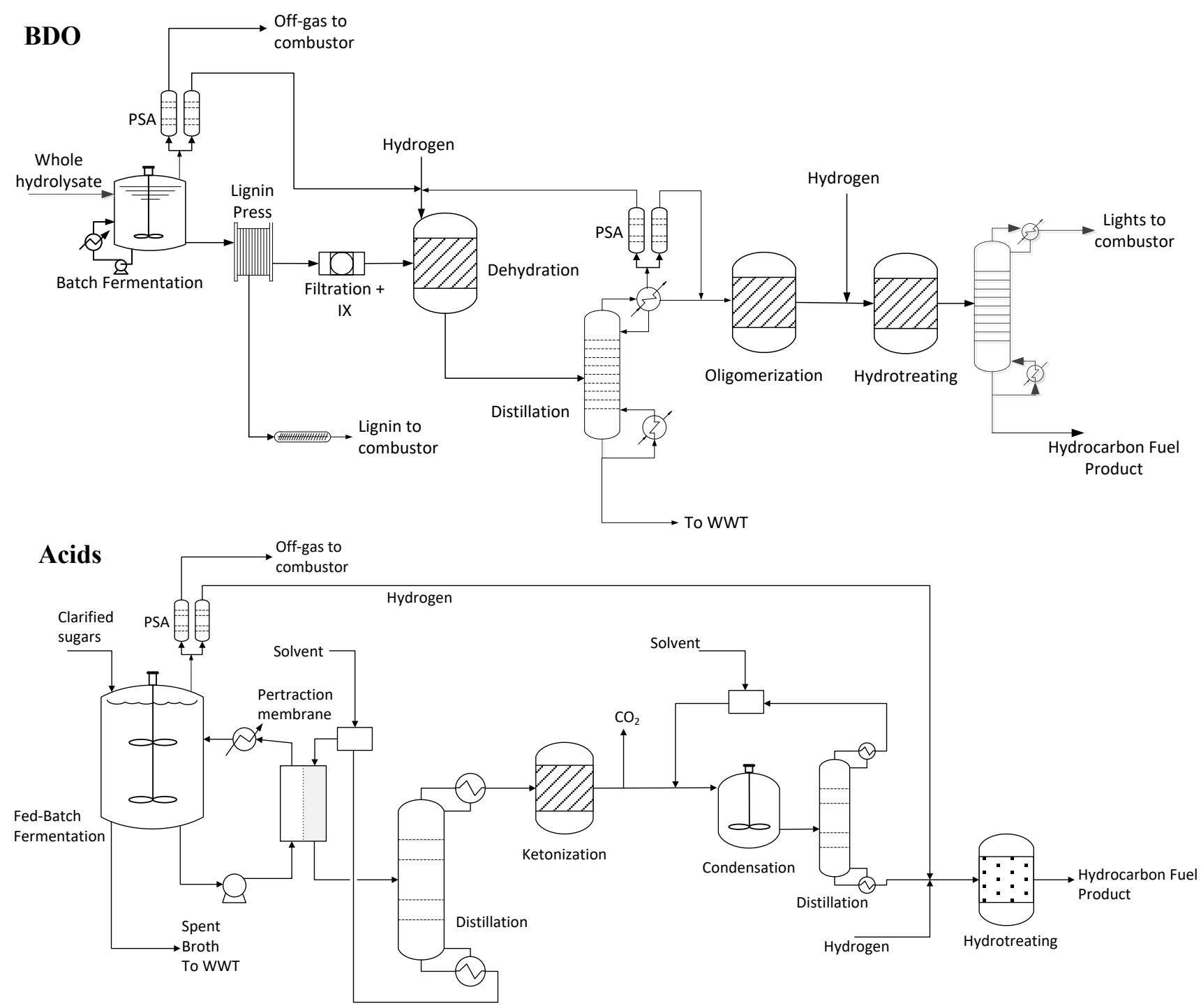

Figure 6. Simplified flow diagram of the fermentation, catalytic conversion, and upgrading process for both fuel train pathways 


\subsubsection{Design Basis}

\section{Anaerobic Fermentation: Background Context}

As introduced in Section 1.1, compared to anaerobic fermentation, aerobic bioconversion is constrained by higher costs for oxygen delivery, both for the equipment to compress and sparge air into the bioreactor media, as well as associated power demands for compression and cooling demands for removing more evolved heat [13]. However, more significant factors are the economy-of-scale penalties for significantly smaller bioreactor volumes $\left(500-1,000 \mathrm{~m}^{3}\right.$ as required for maintaining uniform concentrations of dissolved oxygen and more stringent control of fed-batch fermentations), compared to anaerobic batch bioreactors on the order of $1 \mathrm{MM}$ gal (roughly $4,000 \mathrm{~m}^{3}$ ) [54, 55]. This translates to 4-8 times more bioreactors required for aerobic versus anaerobic fermentation. While access to industry information is sparse with respect to maximum anaerobic fermentor vessel size possible, $1 \mathrm{MM}$ gallon stirred tank vessels are commonly implemented in corn and cellulosic ethanol commercial facilities (feedback from industry).

These drivers contribute to an MFSP premium on the order of \$2/GGE for aerobic bioconversion options for sugars-to-hydrocarbon pathways relative to anaerobic options, as presented earlier in Figure 1. This economy-of-scale penalty translates to a stronger influence of fermentation capital costs for aerobic pathways, which in turn is dependent on achievable fermentation productivity as reflected in Figure 7, with aerobic yeast lipid productivity targets previously set at $1 \mathrm{~g} / \mathrm{L}-\mathrm{hr}$. NREL's latest TEA efforts on aerobic bioconversion assumed the use of $1,000 \mathrm{~m}^{3}$ bubble column bioreactors, which correspond to the largest aerobic bioreactor systems currently deployed commercially; however, recent feedback from a consultancy with Genomatica indicated that potentially up to $2,000 \mathrm{~m}^{3}$ sizes may still be plausible as $n^{\text {th }}$-plant designs for commodity production (thus shrinking economy of scale differences to within $2 \mathrm{X}$ versus anaerobic) [56]. Such large sizes would in fact exacerbate dissolved oxygen variations throughout the reactor, but Genomatica noted that this would not necessarily be insurmountable given sufficient strain development.

Beyond the aerobic cost challenges, the most developed/straightforward pathway that had previously been the primary focus of NREL's (and others') research — intracellular production of lipids via oleaginous yeast -also faced a significant risk in the ability to demonstrate lipid secretion, or at a minimum cell autolysis, as a key future goal in order to avoid significant costs incurred for cell disruption and intracellular lipid extraction. If this goal were not achieved, our TEA estimated an additional cost penalty on the order of roughly $\$ 1.50 /$ GGE (Figure 7), which would be insurmountable in the ability to ultimately achieve under $\$ 2.50 /$ GGE final MFSP targets. While there is limited literature information indicating some success having been achieved for secretion of fatty acid derivatives $[57,58]$, this is generally viewed as a higher-risk hurdle given NREL's latest state of experimental efforts. 


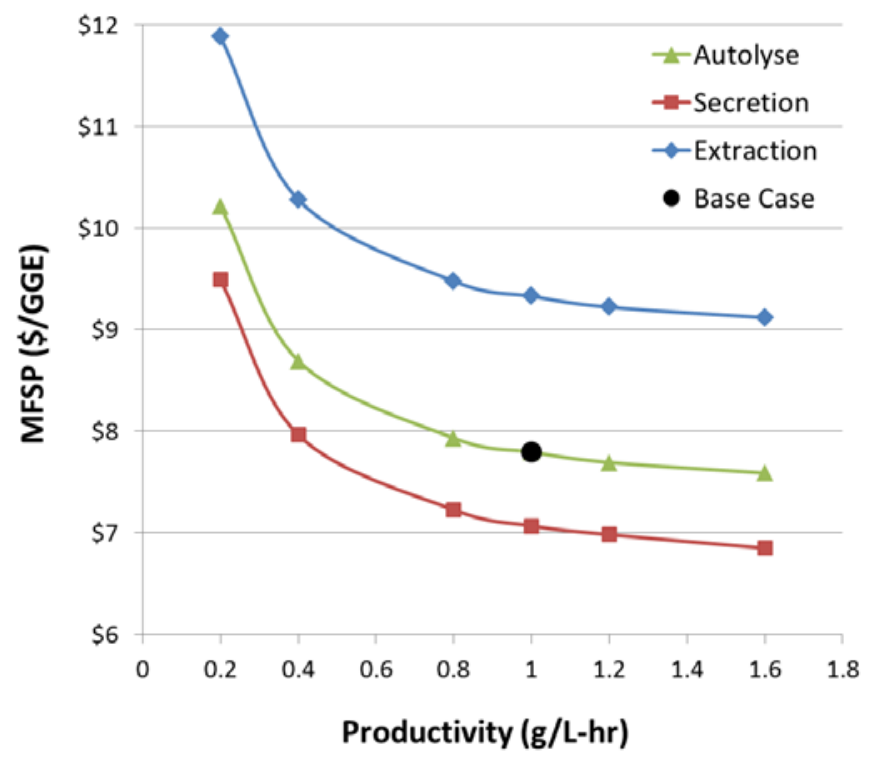

Figure 7. Scan plots for MFSP sensitivities to bioconversion productivity and product recovery method for aerobic lipids pathway (excluding lignin coproducts to reduce MFSP goals per top bars of Figure 1)

To further understand TEA ramifications for aerobic systems, more sophisticated models were built beyond the capabilities in Aspen Plus. Namely, the complex dynamics of operating an aerobic fedbatch bubble column with distinct phases for organism biomass growth versus lipid accumulation (and associated demands for OURs, OTRs, and nutrient feeding strategies) are challenging to accurately reflect in a steady-state process model framework like Aspen Plus. Accordingly, with assistance from an industry collaborator, a new model was built using Aspen Custom Modeler (ACM), which is an equation-based model that enables tracking such dynamic interactions based on engineering equations for oxygen solubilization coupled with a "black-box" metabolic model for oxygen uptake and cell/lipid accumulation (manuscript in preparation).

While the details and the mechanics of the ACM model are beyond the scope of focus for this report, to summarize the findings, a number of scenarios were investigated (Figure 8), including:

a) Validation of prior Aspen Plus TEA estimates for a target case asserting cell autolysis of oleaginous yeast for low-cost lipid recovery (red versus pink curves in Figure 8, under a more narrow range of productivities as considered in Figure 7)

b) Secretion of lipids from the cell under semi-continuous (rather than semi-batch) operation, either with diauxic or simultaneous metabolism of C6 versus C5 sugars (orange curves)

c) Secretion of lipids under semi-batch operation with inclusion of cell recycle back to the bioreactor (lessening the diversion of hydrolysate carbon to new biomass growth; blue curve)

d) Secretion of lipids under semi-batch operation with cell recycle, coupled with increased theoretical metabolic yields under a longer-term future scenario with a highly engineered cell (green curve). 

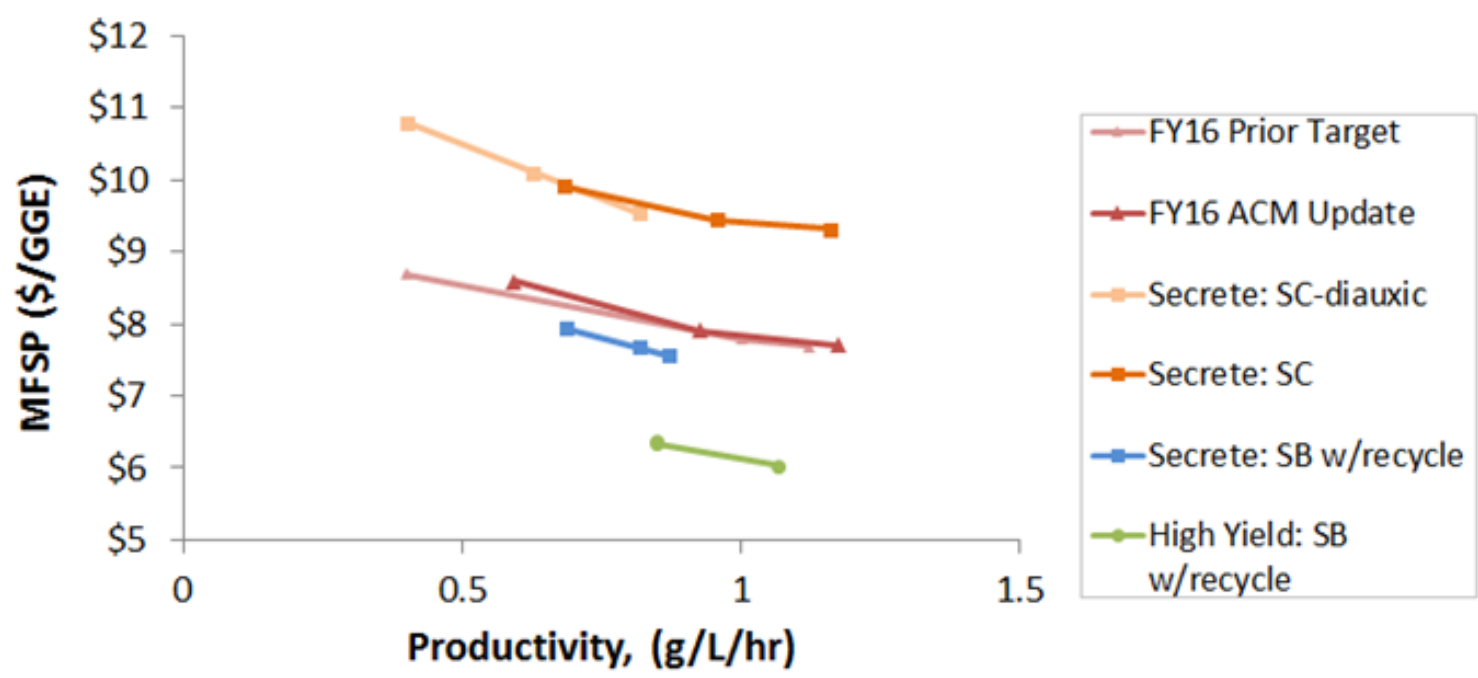

Figure 8. ACM model integrated with Aspen Plus TEA for investigation of aerobic lipid pathway scenarios. $\mathrm{SC}=$ semi-continuous; $\mathrm{SB}=$ semi-batch; recycle = incorporating cell recycle back to bioreactors to minimize carbon diversion from hydrolysate to grow new cell biomass; high yield = increased theoretical metabolic yields to lipids as implied in [59]. All cases prior to inclusion of lignin coproducts to reduce MFSP targets per top bars of Figure 1.

In short, the ACM model was found to confirm similar MFSP estimates as the steady-state Aspen Plus framework, thus further supporting the conclusions drawn around the "aerobic versus anaerobic" TEA tradeoffs discussed above. The ACM model also identified potential paths to further reduce MFSPs, most dramatically under case (d) above, nearly to a level comparable with the anaerobic pathway MFSPs shown earlier in Figure 1, albeit under a combination of targets that would likely require a longer-term research path than what could be more readily achieved under anaerobic fermentation approaches. Thus, for these reasons, the decision was made to focus on the anaerobic pathway options for fuel production in this design case (discussed below).

This is not intended to imply a universal recommendation against aerobic bioconversion in all instances, as many pathways for products, including high-value bioproducts, are inherently aerobic and their processing economics may likely support the higher values garnered by such products relative to commodity fuels. Indeed, both the enzyme train as well as the lignin coproduct train for this design case include aerobic bioconversion steps. Further, while NREL research targets had previously been set at $1 \mathrm{~g} / \mathrm{L}-\mathrm{hr}$ productivity goals for aerobic lipid production, this goal was subsequently achieved earlier than anticipated in 2017, implying further room likely would exist to continue improving on this moving forward into the future (recent industry feedback indicated performance on the order of $2 \mathrm{~g} / \mathrm{L}-\mathrm{hr}$ for certain anabolic products), although moving beyond the $1 \mathrm{~g} / \mathrm{L}-\mathrm{hr}$ level would lead to diminishing economic returns as shown in Figure 7. It is also noted that with the onset of advanced genetic engineering strategies "aerobic" is no longer a universally black-and-white distinction from anaerobic; pathway modifications and redox balancing can enable sizable reductions in oxygen demand compared to the native pathways and promote efficient conversion with very little oxygen [60]. 


\section{Fermentation/Product Recovery: BDO}

The anaerobic xylose and glucose fermenting bacterium $Z$. mobilis can be engineered to redirect carbon from ethanol to produce other products, but still take advantage of its high specific sugar uptake rate, rapid catabolism, and high carbon yield [14]. NREL initiated this effort starting in 2015 by recruiting three genes encoding acetolactate synthase, acetolactate decarboxylase, and butanediol dehydrogenase from Enterobacter cloacae to channel pyruvate to acetolactate, acetoin, and then to 2,3-BDO into Z. mobilis. 2,3-BDO production was demonstrated to reach a titer over $10 \mathrm{~g} / \mathrm{L}$ in 2015 [61], and then $23 \mathrm{~g} / \mathrm{L}$ in 2016 [62] from $10 \%$ glucose in lab medium under low oxygen conditions. At that time, the engineered Z. mobilis strain exhibited a yield from glucose for 2,3-BDO, alongside acetoin and ethanol coproducts, at $96 \%$ of theoretical. In 2017, NREL researchers demonstrated a BDO titer above $40 \mathrm{~g} / \mathrm{L}$ from $10 \%$ glucose and also 42 to $48 \mathrm{~g} / \mathrm{L}$ from glucose and xylose in DMR hydrolysate by knocking out the pyruvate decarboxylase $(p d c)$ gene in Z. mobilis strain $\mathrm{BC} 21$ and eliminating ethanol production [63].

At present, $\mathrm{BDO}$ fermentation experimental work has maintained the use of minimal air intake to the bioreactor (whether by sparging or intake to the headspace) to provide some dissolved oxygen for purposes of organism redox balancing. The minimal aeration level for this purpose is not the same as aerobic fermentation and may be met by merely maintaining oxygen in the fermentor headspace (as has already been demonstrated experimentally). Thus, even if minimal oxygen was still required moving forward, it is expected that the same 1 MM-gal CSTR vessels could be maintained for this pathway (rather than the smaller aerobic bubble column designs and associated economic penalties discussed previously), potentially with minor modifications to ensure proper air supply to the headspace or higher agitation power demands. However, ongoing research efforts are working to engineer the organism to provide the necessary redox balancing in a different way, including coproduction of hydrogen or succinic acid while eliminating oxygen requirements. Co-producing hydrogen while maintaining the same BDO yield would result in marginal cost savings but this modification can mitigate concerns with oxygen requirements in transforming this pathway from a microaerophilic to an anaerobic process. Thus, this modification is targeted as the basis for the projected design case here. Hydrogen is recovered from the fermentor off-gas at high purity via pressure swing adsorption (PSA) for both this and the acids pathway. Any safety apparatus requirements pertinent to this operation (i.e. explosion mitigation measures) are outside the level of granularity in cost estimations performed here, and are assumed to be included in equipment indirect cost factors.

The biological conversion of sugars to BDO can be performed on whole-slurry hydrolysate without the necessity of solid-liquid separation before fermentation. Also, Z. mobilis tolerates a higher concentration of BDO $(>100 \mathrm{~g} / \mathrm{L})$ than ethanol [14], which enables the higher TS loading to be targeted here at $25 \%$ TS through enzymatic hydrolysis and fermentation to achieve such an elevated $\mathrm{BDO}$ titer (modeled here at $97 \mathrm{~g} / \mathrm{L}$ given the targeted conversions and the $25 \%$ TS loading level). This also reduces energy usage and capital expenses for removing water downstream. The process targets overall sugar utilizations of $95 \%, 90 \%$, and $85 \%$ for glucose, xylose, and arabinose, respectively (as well as $95 \%$ utilization of the biomass sucrose), translating to a targeted process yield of $0.47 \mathrm{~g} \mathrm{BDO} / \mathrm{g}$ total sugars, over a 1.5 -day fermentation batch time and $32^{\circ} \mathrm{C}$ fermentation temperature.

Beyond the main fermentation step, the assumptions for seed train design and conversions are maintained consistently with the details discussed in the 2011 ethanol report (utilizing another strain of Z. mobilis), including a $10 \%$ split of hydrolysate to inoculum growth consisting of two trains of five 
reactors each, increasing in size up to 200,000 gal $\left(757 \mathrm{~m}^{3}\right)$ operated in 24-hour batch cycles [2]. Nutrients for all fermentation and inoculum steps are also maintained with prior design cases, represented by corn steep liquor (CSL) and diammonium phosphate (DAP). During peer review of this report, it was noted that in 2017 the use of CSL disqualified advanced biofuel facilities from claiming Renewable Identification Number (RIN) credits. While this study does not include RIN credit considerations in the TEA calculations, we note that alternative nutrient sources (e.g. ammonia) are also possible, and either way would not constitute sizeable contributions to overall fuel selling price at the minimal levels they are utilized here. Additionally, given that the fermentors are not designed for aseptic operation, the same assumptions for contamination losses are maintained as in prior biological design cases, namely 3\% loss of all sugars to lactic acid. All key BDO fermentation conditions and parameters are summarized in Table 9 and Table 10.

Table 9. BDO Fermentation Conditions

\begin{tabular}{lr}
\hline Organism & Recombinant Z. mobilis \\
Temperature & $32^{\circ} \mathrm{C}\left(96^{\circ} \mathrm{F}\right)$ \\
Initial fermentation solids level & $25 \%$ total solids \\
Residence time & 1.5 days $(36 \mathrm{~h})=2.6 \mathrm{~g} / \mathrm{L}-\mathrm{hr}$ productivity \\
Total BDO fermentation volume required & $30,000 \mathrm{~m}^{3}(7.9 \mathrm{MM}$ gal $)$ \\
Number and size of vessels allocated to fermentation & $8 @ 3,600 \mathrm{~m}^{3}(950,000$ gal) each \\
Inoculum level & $10 \mathrm{vol} \%$ \\
Corn steep liquor (CSL) level & $0.25 \mathrm{wt} \%$ \\
Diammonium phosphate (DAP) level & 2 \\
Inoculum production: number of seed trains & 5 \\
Inoculum production: number of batch stages & 5 \\
Inoculum production: maximum stage volume & $0.33 \mathrm{~g} / \mathrm{L}$ fermentation broth (whole slurry) \\
\hline
\end{tabular}

Table 10. BDO Fermentation Reactions and Assumed Conversions

\begin{tabular}{lcc}
\hline Reaction & Reactant & $\begin{array}{c}\text { \% Converted } \\
\text { to Product }\end{array}$ \\
\hline BDO Production: & Glucose & $95.0 \%$ \\
Glucose $\rightarrow 2,3-\mathrm{BDO}+\mathrm{H}_{2}+2 \mathrm{CO}_{2}$ & Glucose & $2.0 \%$ \\
Glucose $+0.047 \mathrm{CSL}+0.018 \mathrm{DAP} \rightarrow 6$ Z. mobilis $+2.4 \mathrm{H}_{2} \mathrm{O}$ & Glucose & $1.0 \%$ \\
Glucose $+2 \mathrm{H}_{2} \mathrm{O} \rightarrow 2 \mathrm{Glycerol}+\mathrm{O}_{2}$ & Xylose & $90.0 \%$ \\
6 Xylose $\rightarrow 52,3-\mathrm{BDO}+5 \mathrm{H}_{2}+10 \mathrm{CO}_{2}$ & Xylose & $1.9 \%$ \\
Xylose $+0.039 \mathrm{CSL}+0.015 \mathrm{DAP} \rightarrow 5 \mathrm{Z}$. mobilis $+2 \mathrm{H}_{2} \mathrm{O}$ & Xylose & $0.3 \%$ \\
3 Xylose $+5 \mathrm{H}_{2} \mathrm{O} \rightarrow 5 \mathrm{Glycerol}+2.5 \mathrm{O}_{2}$ & Xylose & $0.1 \%$ \\
Xylose $+\mathrm{H}_{2} \mathrm{O} \rightarrow \mathrm{Xylitol}+0.5 \mathrm{O}_{2}$ & Arabinose & $90.0 \%$ \\
6 Arabinose $\rightarrow 52,3-\mathrm{BDO}+5 \mathrm{H}_{2}+10 \mathrm{CO}_{2}$ & Arabinose & $1.9 \%$ \\
Arabinose $+0.039 \mathrm{CSL}+0.015 \mathrm{DAP} \rightarrow 5 \mathrm{Z}$. mobilis $+2 \mathrm{H}_{2} \mathrm{O}$ & Arabinose & $0.3 \%$ \\
3 Arabinose $+5 \mathrm{H} \mathrm{H}_{2} \mathrm{O} \rightarrow 5 \mathrm{Glycerol}+2.5 \mathrm{O}_{2}$ & & \\
Seed Train: & Glucose & $4 \%$ \\
Glucose $+0.047 \mathrm{CSL}+0.018 \mathrm{DAP} \rightarrow 6$ Z. mobilis $+2.4 \mathrm{H}_{2} \mathrm{O}$ & Xylose & $4 \%$ \\
Xylose $+0.039 \mathrm{CSL}+0.015$ DAP $\rightarrow 5 \mathrm{Z}$. mobilis $+2 \mathrm{H}_{2} \mathrm{O}$ & & $0.47(0.45)$ \\
\hline Overall BDO process yield (metabolic yield), g/g sugars & & \\
\hline
\end{tabular}

${ }^{a} \mathrm{CSL}$ and DAP are both nitrogen sources required for $Z$. mobilis growth. The stoichiometry shown above is only used to balance the compositions assumed for $Z$. mobilis cell mass.

Following completion of the fermentation batch cycle, the fermentor broth is routed to a clarification step, employing a lignin press to remove lignin and other residual solids, utilizing consistent assumptions as the 2011 ethanol design report for this operation as was used to clarify ethanol beer 
stillage $[2,64,65]$. This step achieves $98 \%$ removal of insoluble solids and reduces water content in the solids material from 80 to $25 \mathrm{wt} \%$; however, it also incurs a small 3\% loss of BDO product. The majority of the Zymomonas biomass is also removed here, which is routed to the boiler and incinerated (as necessary for engineered organism destruction). Similar to the use of the lignin press on the CEH solids stream as discussed above, again it is noted that the nature of DMR-pretreated solids in the present design will make for more challenging filterability through the lignin press (versus DDA pretreatment), and could require the use of a flocculant to achieve reliable operation. As this is not yet well-understood, this will be an important area for further evaluation moving forward.

\section{Catalytic Upgrading: BDO}

Following bulk solids removal from the lignin press, the clarified BDO fermentation broth is further purified across a polishing filter (microfilter) to remove particle fines, followed by ion exchange to remove soluble cations and anions that may otherwise deactivate downstream catalysts. These operations were included here based on subcontractor guidance during NREL's 2015 catalytic upgrading design case focused on catalytic aqueous phase reforming of sugars to fuels [9], where they were deemed necessary to ensure catalyst protection at least in the context of those reactor systems. It is not well-understood whether such operations are also required here for aqueous BDO upgrading, but they were conservatively maintained using consistent design and costing assumptions as employed in the 2015 case. The polishing filter consists of parallel crossflow microfiltration skids with a pore size of 0.1 micron. The filtrate is routed to ion exchange for further purification. A separate-bed ion exchange system is utilized to remove a range of ionic species. Two resin bed trains are included-one for anions and one for cations [66]. There are two units installed in parallel to allow for regeneration of the resin, which is assumed to be required every 17 hours [66]. Resin is regenerated with acid and caustic. While specific components expected to be most problematic for downstream catalysts and their concentration limits are not known, it is expected that a separate-bed system utilizing both anionic and cationic resins will be sufficiently flexible to remove a wide variety of ionic impurities as required for downstream catalyst protection. An additional 1\% loss of BDO is assumed across the combination of these two purification steps. The purified stream is then routed to BDO upgrading.

The 2,3-BDO product may undergo catalytic upgrading either based on high-purity or bulk aqueous catalysis. In the former case, there are several options for separating 2,3-BDO from fermentation broth, including traditional or more novel distillation techniques (i.e., reactive or membrane distillation), aqueous two-phase reforming, in situ recovery, integrated solvent extraction and pervaporation, steam stripping, reaction with formaldehyde, and vacuum membrane filtration [67, 68]. Standard distillation is the most straightforward approach for handling pertinent separations, but incurs significant energy penalties as the boiling point of 2,3-BDO (roughly $180^{\circ} \mathrm{C}$ ) is higher than that of water, thus would require boiling all the water off from BDO [69]. To alleviate this, the liquor may first be concentrated by a vacuum evaporator to remove some of the water, which could be driven by power rather than heat for this step if utilizing an MVR evaporator. Subsequently the concentrated stream would be sent through two sequential distillation towers to separate and purify 2,3-BDO from the remaining water and residual soluble solids. The final BDO product $(>92 \%)$ could then be catalytically upgraded to butenes or butadiene (e.g., using $\mathrm{CsH}_{2} \mathrm{PO}_{4}-\mathrm{SiO}_{2}$ catalyst). Overall, this would be a highly energy-intensive option.

Alternatively, aqueous catalytic upgrading could use the full clarified fermentation broth directly without BDO purification. This approach could simplify the overall process concept by eliminating the BDO concentration/distillation steps noted above, although still would require heating (albeit not 
boiling) the aqueous stream up to reaction temperatures and processing a larger aqueous throughput through the BDO upgrading reactor. Oak Ridge National Laboratory (ORNL) reported a technology converting ethanol and ethanol-water mixtures to hydrocarbon fuels over zeolite-based catalysts, which were shown to be robust in tolerating water content between $5 \mathrm{wt} \%$ and $95 \mathrm{wt} \%$, as well as volatile impurities in the feed stream [70].

More recently in 2017, preliminary catalytic upgrading experiments were performed by ORNL researchers as proof-of-concept for similar aqueous conversion of diols, with similarly effective conversion using core-shell $\mathrm{SiO}_{2} @ \mathrm{ZrO}_{2}$ catalysts. The products from this aqueous catalytic reaction were a mixture of butenes, methyl ethyl ketone (MEK), butadiene, $\mathrm{C} 2-\mathrm{C} 3$ olefins, 2-methy propanal, acetone, and other minor components. The butene product would still require purification prior to subsequent oligomerization reactions but poses an easier separation since all products at that stage boil lower than water. This aqueous BDO upgrading approach is the basis considered in the present design configuration, based on inputs provided by ORNL collaborators for target projections on BDO fermentor broth [71].

Catalytic upgrading of 2,3-BDO to olefins involves cascade reactions, such as dehydration, hydrogenation, rearrangement, and other side reactions. Dehydration of 2,3-BDO leads to MEK and 1,3-butadiene. Rearrangement and dehydration reactions produce 2-methyl propanal. Product selectivity between these dehydration intermediates depends primarily on the type of acidic catalyst. Selective hydrogenation of carbonyl and 1,3-butadiene while maintaining olefins is challenging. Metal catalysts with mild hydrogenation properties (e.g. $\mathrm{Cu}$ ) are needed this type of selective reaction. Bifunctional catalysts with dehydration and hydrogenation activity are key for one-step upgrading of 2,3-BDO to olefins (Figure 9). Depending on the type of catalyst employed, other side reactions are possible, such as coupling of butenes and cracking reactions.

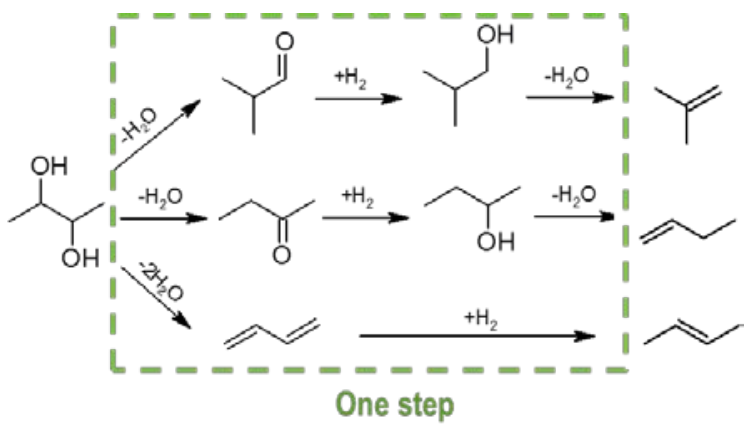

Figure 9. 2,3-BDO upgrading reaction pathways to olefins

For 2,3-BDO aqueous catalytic upgrading, bifunctional solid acid catalysts are used. In the present design, the aqueous BDO stream containing roughly $8 \mathrm{wt} \% \mathrm{BDO}, 91 \mathrm{wt} \%$ water, and $1 \mathrm{wt} \%$ other components (primarily unconverted sugars) is converted adiabatically to dehydration intermediates over copper-based catalysts (e.g., $\mathrm{Cu} / \mathrm{SiO}_{2}-\mathrm{ZrO}_{2}$ and $\mathrm{Cu} /$ zeolite) at $250^{\circ} \mathrm{C}$ and $45 \mathrm{~atm}[64,65,72]$. As shown in Table 11, 100\% of the $\mathrm{BDO}$ is converted to a number of representative components, namely butenes, pentenes, hexenes, MEK, and other minor C2-C3 components. Stoichiometric element balances are closed with hydrogen consumption and water formation, with model convergence based on the NRTL property package given the presence of oxygenated/polar components (this is the default property package in most of the biochemical model steps for both fuel train pathways). 
The composition of products is dependent on the composition of the feed stream, the reaction temperature and pressure, space velocity, hydrogen/BDO ratio, and catalyst type. When the temperature decreases to $200^{\circ} \mathrm{C}$, the selectivity of $\mathrm{C} 3+$ olefins drops to $60 \%$ while $\mathrm{MEK}$ approaches $40 \%$. Space velocity also has a dramatic effect on the product selectivity. When weight hourly space velocity (WHSV) increases from 0.5 to $5.0 \mathrm{~h}^{-1}, \mathrm{C} 3+$ olefins selectivity decreases and MEK selectivity increases.

Aside from this information, data in the public domain remains scarce on the topic of aqueous catalytic upgrading of 2,3-BDO to hydrocarbons, particularly in the context of biomass hydrolysate fermentation broth. Moving forward, to achieve the design case targets, continued focus on designing active and robust catalysts for aqueous BDO conversion at high selectivity will be important, as well as understanding (and if needed, minimizing) the impact of impurities in BDO fermentation broth on catalyst performance.

Table 11. Product Distribution of the 2,3-BDO Upgrading Reaction (Future targets at high conversion and selectivity to desired alkenes)

\begin{tabular}{|l|c|c|c|c|}
\hline & Group & C percent, mol\% & Product mol\% & Yield mol\% \\
\hline Propene & C3 & 9.78 & 3.26 & 4.45 \\
\hline 1-butene & $\mathrm{C} 4$ & 8.13 & 2.03 & 2.78 \\
\hline cis-2-butene & $\mathrm{C} 4$ & 14.83 & 3.71 & 5.06 \\
\hline trans-2-butene & $\mathrm{C} 4$ & 25.53 & 6.38 & 8.72 \\
\hline n-pentene & $\mathrm{C} 5$ & 13.20 & 2.64 & 3.61 \\
\hline n-hexene & $\mathrm{C} 6$ & 3.80 & 0.63 & 0.86 \\
\hline Propane & $\mathrm{C} 3$ & 1.36 & 0.45 & 0.62 \\
\hline Water & $\mathrm{H}_{2} \mathrm{O}$ & & 48.28 & 65.93 \\
\hline Isobutane & $\mathrm{C} 4$ & 0.87 & 0.22 & 0.30 \\
\hline Isobutylene & $\mathrm{C} 4$ & 15.60 & 3.90 & 5.33 \\
\hline MEK & $\mathrm{C} 4$ & 6.88 & 1.72 & 2.35 \\
\hline
\end{tabular}

The 2,3-BDO upgrading reactor cost is based on the aqueous phase reforming (APR) reactor in NREL's 2015 catalytic upgrading design case [66], sized based on the total aqueous flow rate into the reactor. The reactor operation consists of packed-bed pressure vessels, clad in 317L SS, and includes internals that support the catalyst and distribute the process fluid. Hydrogen is added to the reactors at a hydrogen-to-BDO ratio of 7.2 (molar basis). This reaction is assumed to convert near $100 \%$ of the 2,3-BDO (and/or ethanol if needed), with a WHSV of $2.0 \mathrm{hr}^{-1}$ and biannual catalyst replacement. The key reaction parameters are summarized in Table 12. Costs for this and all catalysts are presented in Section 4.3. As discussed previously, catalyst performance can be sensitive to a number of impurities that could pose challenges to catalyst lifetime, such as sulfur, cations, anions, solids, and coke [73]. The effect of remaining substrates (depending on the concentration) on the catalyst stability should be further evaluated in the future R\&D work. A challenge for aqueous BDO upgrading is catalyst stability in the condensed water phase (acid catalyst support and metal leaching). 
Table 12. 2,3-BDO Catalytic Upgrading Reactor Conditions

\begin{tabular}{lc}
\hline Parameter & Operating Condition \\
\hline Hydrogen Molar Ratio & $7.2 \mathrm{~mol} \mathrm{H}_{2} / \mathrm{mol} \mathrm{BDO}^{\circ}$ feed \\
Operating Temperature & $250^{\circ} \mathrm{C}$ \\
Operating Pressure & $45 \mathrm{~atm}$ \\
WHSV & $2.0 \mathrm{hr}^{-1}$ \\
Catalyst Type & Copper-based catalysts $\mathrm{Cu} / \mathrm{SiO}_{2}-\mathrm{ZrO}_{2}, \mathrm{Cu} /$ zeolite \\
Catalyst Lifetime & 2 years \\
\hline
\end{tabular}

The product of the BDO catalytic upgrading step is sent to distillation to remove water. The distillation is more straightforward for butene, which is a gas at ambient conditions, versus purification of BDO, which boils above water as noted previously. The other olefin products from BDO upgrading also boil lighter than water. Heat integration is done by cross-exchange between the hydrodeoxygenation (HDO) reactor feed and the butene distillation column bottoms product. A trim heater is placed after this exchanger on the BDO feed line to further heat the BDO stream to the reactor inlet temperature of $250^{\circ} \mathrm{C}$.

The butene column overhead product containing the olefin components is routed through oligomerization to C8-C16 oligomers. The oligomerization step is modeled using a fixed bed reactor operated at $150^{\circ} \mathrm{C}$ and $15 \mathrm{~atm}$, containing Amberlyst-36 resin at a cost of $\$ 71 / \mathrm{lb}(2014 \$)$, with a target WHSV of $1 \mathrm{~h}^{-1}$. The butenes are oligomerized with a 28\%, 63\%, and $9 \%$ selectivity to butene dimers $\left(\mathrm{C}_{8}\right)$, trimers $\left(\mathrm{C}_{12}\right)$, and tetramers $\left(\mathrm{C}_{16}\right)$, respectively [74]. Pentene and hexene are oligomerized to dimers $\left(\mathrm{C}_{10}\right.$ and $\left.\mathrm{C}_{12}\right)$. The conversions of propene and butene are assumed to be $95 \%$ based on literature data [75-77], while the conversions of C5 and C6 olefins are assumed to be $100 \%$ in the same oligomerization reactor. In the future, the oligomerization reaction conditions and product distributions may be refined as further inputs/data from bench experiments become available. The oligomerization is designed and priced as a pressurized reactor vessel with fixed beds for Amberlyst36 resin catalysts. The key parameters for the oligomerization step are summarized in Table 13.

Table 13. Oligomerization Reactor Conditions

\begin{tabular}{lc}
\hline Parameter & Operating Condition \\
\hline Operating Temperature & $150^{\circ} \mathrm{C}$ \\
Operating Pressure & $15 \mathrm{~atm}$ \\
WHSV & $1.0 \mathrm{hr}^{-1}$ \\
Catalyst Type & Amberlyst-36 resin \\
Catalyst Lifetime & 2 years \\
\hline
\end{tabular}

After oligomerization, a hydrotreating step is used to saturate the remaining double bonds to finished paraffinic hydrocarbon fuels. In this step, the feed stream is mixed with fresh and recycled hydrogen from the process, and then heated to an inlet reactor operating temperature of $371^{\circ} \mathrm{C}$ and a pressure of $200 \mathrm{psig}$, [78]. As this is a relatively straightforward step for this pathway (hydrogenation), but is otherwise outside the scope of planned experimental work at NREL or ORNL, the model currently assumes similar design and cost details as the final hydrotreating step for the acids case, and a severity equivalent to a typical petroleum naphtha hydrotreater with a WHSV of $5 \mathrm{hr}^{-1}$ utilizing a $5 \% \mathrm{Pd} / \mathrm{C}$ catalyst [79-82]. A final distillation column fractionates the hydrocarbon products from the light gases, with the latter routed to the boiler. 


\section{Fermentation/Product Recovery: Acids}

The production of carboxylic acids was identified in early 2015 as a potential strategy to anaerobically produce a biological intermediate that could be further upgraded to a hydrocarbon fuel via chemical catalytic conversion. Initial exploration focused on biological production of hexanoic acid via Megasphaera elsdenii, a natural lactic acid utilizing bacteria isolated from cow ruminant that has been shown to naturally produce acetic (C2), butyric (C4), valeric (C5), and hexanoic (C6) acids [79-83]. Given challenges associated with the conversion of xylose, the focus shifted to prospecting other potential hosts for improved production and conversion at the cost of acid chain length.

Subsequent downselection of $\mathrm{C} 2-\mathrm{C} 4$ producing anaerobic strains led to three strong candidates, Clostridium butyricum (ATCC 19398), Clostridium tyrobutyricum (ATCC 25755), and Clostridium carboxidivorans $P 7$ (ATCC BAA-624), which all showed naturally high carbon diversion to acid products. However, a key challenge with this approach is the product toxicity at high titers which mandates $\mathrm{pH}$ control (adding large costs to the fermentation) or continuous removal of acids as they are produced [84-86]. Recent NREL R\&D efforts have demonstrated the ability to produce acetic and butyric acids at high overall fermentation performance on both $\mathrm{C} 5$ and C6 sugars (i.e., high yields at $0.45 \mathrm{~g} / \mathrm{g}$ sugars with nearly $100 \%$ overall utilization of major sugars and $1.1 \mathrm{~g} / \mathrm{L}-\mathrm{hr}$ productivity using Clostridium butyricum in batch fermentation) with concurrent production of hydrogen.

Biological acid toxicity/inhibition is a well-known phenomenon, and similar to ethanol or BDO toxicity the titer of the product species often determines the onset of cell inhibition. Differing from alcohols, an acid product has the added contribution of $\mathrm{pH}$ which can modulate the "apparent" titer of the toxic protonated acid form and affect the redox state of the host cell prompting metabolic shifts in certain cases [87]. Concurrent to the down-selection process a biological and a process strategy was pursued to overcome this toxicity barrier. Biologically, natural strains vary dramatically in their ability to tolerate titers and $\mathrm{pH}$; therefore, the NREL R\&D team pursued a campaign to identify genetically tractable, acid-tolerant organisms (with high tolerance to organic acids specifically), capable of a broad sugar utilization range and with high tolerance to biomass hydrolysates. While this effort is ongoing and has identified multiple strains of interest possessing several of the desired traits, to date no strains demonstrate all of the desired characteristics. Accordingly, efforts have focused on engineering several acid-tolerant yeast species for expanded sugar utilization capabilities and elevated tolerance to butyric acid in low $\mathrm{pH}$ environments.

From a process perspective, operating fermentations with in situ product removal minimizes accumulation of toxic products in the broth and hence avoids cell inhibition [88-93]. Furthermore, in situ product removal has no inherent upper limit on productivity/rate since the removal rate of acid products is a function of the extraction unit sizing, not an underlying enzymatic/physiological mechanism. Finally, operating at low extracellular product titers promotes higher secretion/excretion rates driving a stronger productivity throughout the fermentation. Despite the advantages in situ product removal systems provide, recovery of acetic acid - in particular across a pertractive membrane - has proven challenging to date, resulting in elevated carbon losses in cases that include this acid component.

Metabolic modeling work indicates a $\mathrm{Fd}-\mathrm{H}_{2}$ cofactor in Clostridia that is used to maintain redox balance when producing butyric acid $[94,95]$. Presence of this cofactor provides a feasible metabolic engineering approach to eliminating acetic acid production, increasing hydrogen yield, and enabling better product recovery of butyric acid alone with an in situ system. As an added benefit, hydrogen 
released during fermentation can be recovered and used in downstream catalytic reactions. For the current design, a recombinant $C$. tyrobutyricum strain engineered to anaerobically produce butyric acid as well as hydrogen from clean DMR hydrolysate is assumed, with the stoichiometries and conversions shown in Table 14.

Table 14. Acids Pathway Stoichiometry and Key Conversions

\begin{tabular}{lcc}
\hline Reaction & Reactant & $\begin{array}{c}\text { \% Diverted to } \\
\text { Reaction }\end{array}$ \\
\hline Glucose +0.018 DAP +0.37 Nutrient $\rightarrow 6$ C. tyrobutyricum $+2.4 \mathrm{H}_{2} \mathrm{O}$ & Glucose & $5 \%$ \\
Glucose $\rightarrow 1$ Butyric $+2 \mathrm{CO}_{2}+2 \mathrm{H}_{2}$ & Glucose & $95 \%$ \\
Xylose $+0.015 \mathrm{DAP}+0.3087 \mathrm{Nutrient} \rightarrow 5$ C. tyrobutyricum $+2 \mathrm{H}_{2} \mathrm{O}$ & Xylose & $15 \%$ \\
6 Xylose $\rightarrow 5$ Butyric $+10 \mathrm{CO}_{2}+10 \mathrm{H}_{2}$ & Xylose & $85 \%$ \\
Arabinose $+0.015 \mathrm{DAP}+0.37 \mathrm{Nutrient} \rightarrow 5$ C. tyrobutyricum $+2 \mathrm{H}_{2} \mathrm{O}$ & Arabinose & $2 \%$ \\
6 Arabinose $\rightarrow 5$ Butyric $+10 \mathrm{CO}_{2}+10 \mathrm{H}_{2}$ & Arabinose & $85 \%$ \\
Sucrose +0.036 DAP $+0.7404 \mathrm{Nutrient} \rightarrow 12$ C. tyrobutyricum $+3.8 \mathrm{H}_{2} \mathrm{O}$ & Sucrose & $5 \%$ \\
Sucrose $\rightarrow 1.9$ Butyric $+4.4 \mathrm{CO}_{2}+5 \mathrm{H}_{2}$ & Sucrose & $95 \%$ \\
\hline
\end{tabular}

${ }^{a} \mathrm{CSL}$ and DAP are both nitrogen sources required for growth. The stoichiometry shown above is only used to balance the compositions assumed for cell mass.

The biological conversion of sugars to butyric acid operates in fed batch using clarified and concentrated sugars exiting the continuous enzymatic hydrolysis system. Given the removal of solids upstream through $\mathrm{CEH}$, the use of clarified sugars reduces membrane fouling and improves mixing hydrodynamics, which minimizes the occurrence of low $\mathrm{pH}$ or high titer pockets in the broth (leading to cell death/inhibition). The use of in situ acid removal across the pertraction membrane may theoretically allow for moving to continuous or semi-continuous modes of fermentation operation, which would improve capital utilization efficiency of the fermentation vessels. However, as membrane lifetimes, back-extraction of solvent, and other dynamic interactions with the fermentor broth over extended time periods are not yet well-understood, such extended fermentation modes are not presently assumed here. The assumptions for seed train design and conversions are maintained consistently with the details discussed in the 2011 ethanol report, including a $10 \%$ split of feed sugar substrate to inoculum growth, a five stage 10:1 volume increase per stage up to 200,000 gal $\left(757 \mathrm{~m}^{3}\right)$ prior to production reactor transfer, and two seed trains. This may be conservative for the present design utilizing concentrated hydrolysate with fewer production fermentor vessels and may be revisited in future design updates, potentially based on the use of the ACM models discussed above to provide a more realistic understanding of the dynamics of cell growth between the seed and production vessels and tradeoffs between feed sugar splits versus inoculum concentration in the production stage.

Fermentation occurs in large $1 \mathrm{MM}$-gal vessels and each production tank has a cooler to maintain temperature at a constant $37^{\circ} \mathrm{C}$ and an agitator to maintain cells in suspension. In situ product removal occurs continuously; as the fermentation progresses acid product is removed using a liquid-liquid membrane extraction system (membrane pertraction) connected through a pump-around loop. Targeted productivity remains high throughout the fermentation at $2.0 \mathrm{~g} / \mathrm{L}-\mathrm{hr}$ acid production (based on targets set by NREL researchers), requiring 3 fermentor vessels. Relative to the ethanol design case (and the basis maintained for BDO fermentation) which minimized agitation power to keep the broth in suspension, this design triples the agitator power demand from 0.15 to $0.45 \mathrm{hp}$ per 1,000 gal (88.6 $\mathrm{KW} / \mathrm{MM} \mathrm{L}$ ), given stronger organism sensitivity to product (acid) titer in the broth and thus a need to maintain better homogeneity as the broth circulates through the membrane. Alternatively, or perhaps in addition to this adjustment, a smaller vessel size may plausibly also be required for the same reason, 
but this is not yet well understood until more detailed design/operational parameters are established for the acids fermentation/membrane pertraction system.

The process assumes complete consumption of glucose, xylose, arabinose, and sucrose, of which smaller fractions are converted to the acids products as shown in Table 14, resulting in an overall process fermentation yield of $0.433 \mathrm{~g}$ butyric/g sugar, equivalent to $89 \%$ of the maximum theoretical metabolic yield. The key carboxylate fermentation metrics are listed in Table 15 below.

Table 15. Key Butyric Acid Fermentation Parameters

\begin{tabular}{ll}
\hline Organism & Clostridium tyrobutyricum \\
Temperature & $37^{\circ} \mathrm{C}\left(99^{\circ} \mathrm{F}\right)$ \\
Productivity & $2.0 \mathrm{~g} / \mathrm{L}-\mathrm{hr}$ \\
Fermentation vessel volume & $3,785 \mathrm{~m}^{3}(1 \mathrm{MM} \mathrm{gal})$ \\
Fermentation vessel agitator power demand & $0.45 \mathrm{hp} / 1,000 \mathrm{gal}(88.6 \mathrm{KW} / \mathrm{MM} \mathrm{L})$ \\
Corn steep liquor seed requirement & $1.3(\mathrm{~g} / \mathrm{g} \mathrm{feed})$ \\
Corn steep liquor production requirement & $0.66(\mathrm{~g} / \mathrm{g}$ feed $)$ \\
Diammonium phosphate seed requirement & $8 \mathrm{~g} / \mathrm{L} \mathrm{fermentation} \mathrm{broth}$ \\
Diammonium phosphate production requirement & $0.495 \mathrm{~g} / \mathrm{L}$ fermentation broth \\
Inoculum production: number of seed trains & 2 \\
Inoculum production: number of batch stages & 5 \\
Inoculum production: maximum stage volume & $200,000 \mathrm{gal}\left(757 \mathrm{~m}^{3}\right)$ \\
Fermentation process yield (g butyric/g sugar $)$ & 0.433 \\
\hline
\end{tabular}

The pertractive in situ product removal system relies on a tri-octyl-phosphine oxide (TOPO)-assisted organic phase to selectively remove acid from the broth across a membrane [96, 97]. Short to medium chain acids show relatively low partitioning to the organic phase due to their significant polarity. To overcome this poor separation, TOPO, which is a strong hydrogen bond acceptor with poor water solubility, is added to the organic phase. The addition of TOPO causes complexation with the protonated acid species and functions as a "carrier" molecule increasing the recovery for shorter acid species. Because complexation is tied to the protonated species exclusively, the efficacy is governed by a multispecies $\mathrm{pH}$-dependent equilibria with effectiveness a function of $\mathrm{pKa}$ (i.e., chain length) and process $\mathrm{pH}$ (i.e., strain $\mathrm{pH}$ toxicity) as shown in Figure 10 [98].
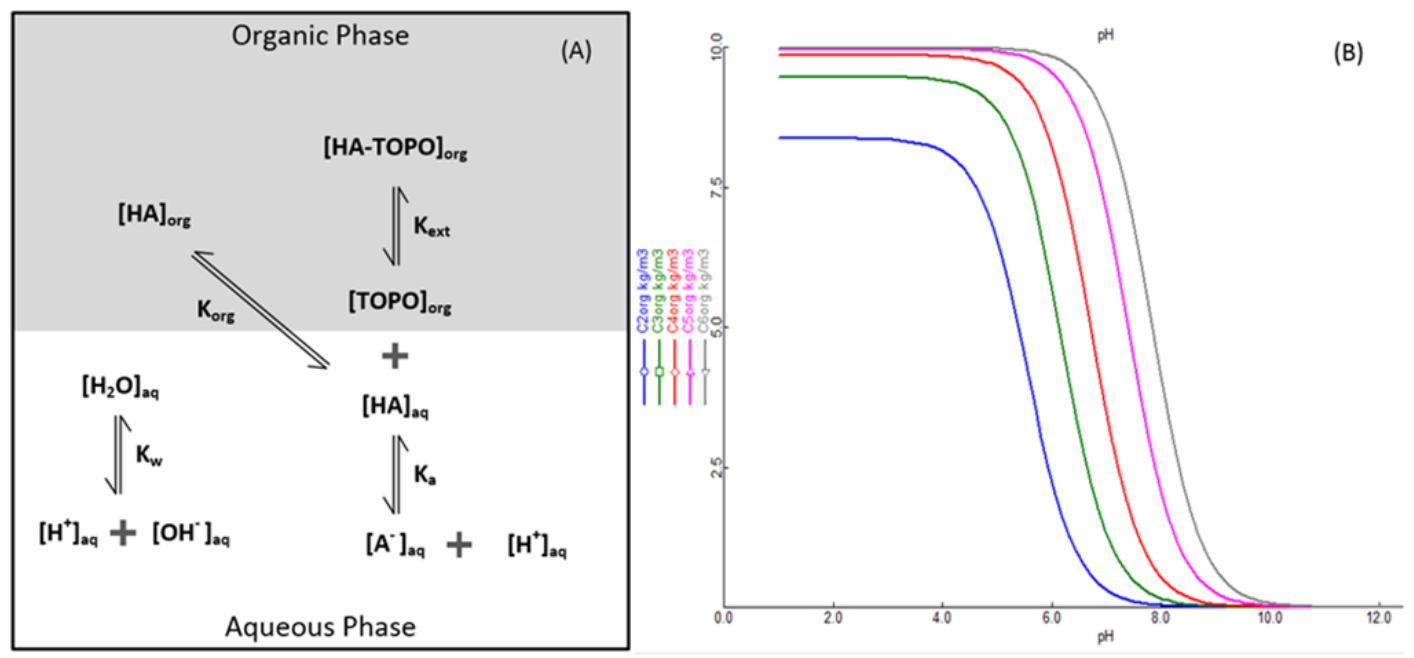

Figure 10. Acid extraction equilibrium and predicted recovery in unit 
The design case uses a liquid-liquid membrane contactor, wherein an aqueous broth phase is fed through the shell side and put in contact with hydrophobic polymer membrane fibers carrying a mineral oil + TOPO organic phase on the tube side. A mild shell-side pressure maintains intimate contact relying on hydrophobic forces to prevent aqueous breakthrough to the organic tube side. The flow rate of the organic phase can be tuned to control the rate of product removal as needed based on the fermentation productivity, as well as target a specific broth acid titer $/ \mathrm{pH}$. In the current design, $C$. tyrobutyricum is assumed to tolerate a $\mathrm{pH}$ of 4.7-5 in the broth, conditions at which the modeled extraction equilibrium predicts a recovery of $100 \%$ of the butyric acid in the aqueous phase (but as noted previously a lower recovery of any acetic acid).

After extraction to the organic phase, a vacuum is pulled on the mixture and it is routed to distillation for acid purification. The vacuum distillation is critical to prevent dimerization and degradation of the TOPO carrier molecule as well as reduce the boiling point improving the energy efficiency of the integrated plant. The acid is recovered in high purity from the distillate while the mineral oil and TOPO exits from the bottom and is recycled back to a storage tank for reuse in the pertractive unit. Table 16 lists the key pertraction metrics and design assumptions.

Table 16. Petraction Operating Specifications

\begin{tabular}{lc}
\hline Parameter & Operating Condition \\
\hline Membrane type & Hydrophobic \\
Operating temperature & Ambient \\
Operating pH & 4.7 \\
Transmembrane pressure & $\sim 15$ psig \\
Solvent & Mineral oil \\
Butyric acid recovery & $100 \%$ \\
\hline
\end{tabular}

\section{Catalytic Upgrading: Acids}

The recovered butyric acid exiting the column is re-pressurized to atmospheric pressure then routed to the catalytic upgrading process. This three-step conversion process consists first of acid condensation/ketonization, followed by ketone condensation to higher molecular weight enones, and finally upgrading of enone intermediates to drop-in hydrocarbon fuels [99]. The additional condensation step allows the pathway to target diesel- and jet-fuel range branched hydrocarbons with a blendstock in the 14-carbon chain length range. The conversion steps are illustrated in Figure 11 with further details provided below. 


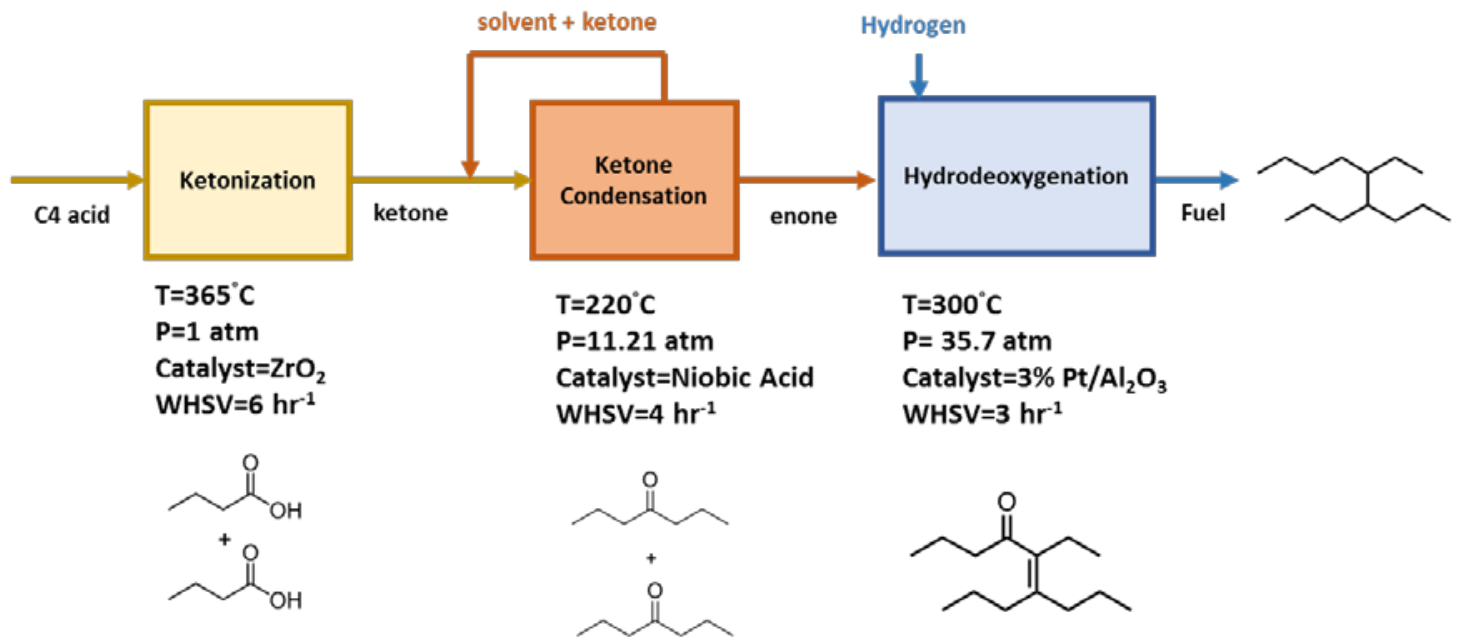

Figure 11. Acid upgrading reaction steps with key metrics

The first step of the process converts the butyric acid to a range of ketones under ambient pressures and elevated temperature $\left(365^{\circ} \mathrm{C}\right)$ [100]. The upstream distillation yields nearly pure butyric acid, which is completely converted to 4-heptanone over an acidic $\mathrm{ZrO}_{2}$ catalyst. The condensation releases one mol of carbon dioxide per two mols butyric acid resulting in a net yield of $0.65 \mathrm{~g} 4$-heptanone/g butyric acid feed and proceeds rapidly with a WHSV of $6 \mathrm{hr}^{-1}$ and a 2-year catalyst lifetime. Heat integration between the effluent and feed reduces the energy demand and a hot oil heating system served by a direct fired heater is used to achieve the high-temperature feed setpoint.

After ketone condensation the product stream is cooled to $50^{\circ} \mathrm{C}$ then flashed to separate the $\mathrm{CO}_{2}$ from the liquid phase with roughly $17 \%$ of the 4 -heptanone intermediate volatilized to the vapor phase during the flash. The 4-heptanone lost in the vapor stream exiting the flash is ultimately recovered via a water scrubber followed by a decanter for phase separation. This recovered 4-heptanone is then recombined with the flash liquid stream resulting in an overall $96.5 \%$ recovery of the acid condensation intermediate for further upgrading. Table 17 shows the key specifications for the initial ketonization reaction.

Table 17. Ketonization Reaction Parameters

\begin{tabular}{lc}
\hline Parameter & Operating Condition \\
\hline Acids-to-Ketones (wt\% acids) & $100 \%$ \\
Operating Temperature & $365^{\circ} \mathrm{C}$ \\
Operating Pressure & $1 \mathrm{~atm}$ \\
WHSV & $6.0 \mathrm{hr}^{-1}$ \\
Catalyst Type & $\mathrm{ZrO}_{2}$ \\
Catalyst Lifetime & $2 \mathrm{yr}$ \\
Overall Ketone Recovery (wt $\%)$ & $96.5 \%$ \\
\hline
\end{tabular}

To produce a diesel- and jet-fuel range blendstock with improved cold flow properties, the seven carbon ketone intermediate is further upgraded via condensation which promotes branching and extends the hydrocarbon to a C14-length enone [101, 102]. Since 4-heptanone is a less reactive central ketone component, to boost the single-pass conversion through condensation the conversion step requires the addition of a heterogeneous catalyst (niobic acid) and a solvent to mitigate catalyst 
inhibition over a longer 15-hour residence time in a stirred tank slurry reactor. In the current design, the liquid feed is pressurized to $150 \mathrm{psig}$ and mixed with toluene to achieve a 3.7:1 solvent-to-ketone ratio $(\mathrm{w} / \mathrm{w})$. Toluene displays beneficial solvent properties associated with the stable aromatic structure which prevents and minimizes solvent cracking to light hydrocarbons at the reaction conditions compared to a similar linear alkane equivalent. Catalyst is added in sufficient quantity to achieve a 1:4 catalyst-to-ketone ratio by weight and the system is heated to $180^{\circ} \mathrm{C}$. The reaction proceeds slowly in a CSTR sized to achieve a mean residence time of 15 hours. At these conditions, the single-pass conversion is $60 \%$ with $100 \%$ conversion to the $\mathrm{C} 14$ enone. The reaction produces 1 mol of water per mole of enone, which phase separates cleanly along with the solid catalyst and is filtered to remove the aqueous solution then recycled for reuse. The enone, toluene, and unreacted ketone are then distilled, separating the lighter boiling toluene solvent and ketone as distillate, which is recycled back to the reactor feed and mixed with the pure acid condensation feed until extinction. The heavy $\mathrm{C} 14$ enone exits the column bottoms along with minor amounts of the 4-heptanone at a temperature of $344^{\circ} \mathrm{C}$. To reduce energy use and heat demands, the distillation is performed at a lower $73 \mathrm{psig}$, though even at reduced pressures the high boiling point enone mandates use of the hot oil system for the reboiler. Table 18 lists the key operating specifications for the ketone condensation step.

Table 18. Ketone Condensation Reaction Parameters

\begin{tabular}{lc}
\hline Parameter & Operating Condition \\
\hline Ketones-to-Enones Conversion (\% ketones feed) & $60 \%$ \\
Operating Temperature & $180^{\circ} \mathrm{C}$ \\
Operating Pressure & $150 \mathrm{psig}$ \\
Catalyst: Ketone Loading (w/w) & 0.25 \\
Solvent: Ketone Loading (w/w) & 3.65 \\
WHSV & $15 \mathrm{hr}$ CSTR \\
Catalyst Type & niobic acid \\
Catalyst Lifetime & 2 years \\
\hline
\end{tabular}

Following ketone condensation, the product enters a fuel finishing step, where the enone intermediate (and any unconverted ketone and solvent intermediates) are fully deoxygenated over a $3 \% \mathrm{Pt} / \mathrm{Al}_{2} \mathrm{O}_{3}$ catalyst in the presence of hydrogen at a pressure of $35 \mathrm{~atm}$. Makeup hydrogen is fed to achieve a 24.6:1 molar ratio to the oxygenated feed entering the reactor. The reactor achieves complete hydrogenation to saturated hydrocarbon products operating at a WHSV of $3 \mathrm{hr}^{-1}$. The product stream is cooled, and excess hydrogen is recovered in a cold high-pressure separator, then recompressed and recycled to the hydroprocessing unit. The $\mathrm{C} 14$ branched diesel blendstock is recovered then sent to storage. As noted previously, hydrogen produced during the fermentation is separated from the other vent vapors via pressure swing adsorption and fed to the hydrotreater. In the current design the butyric acid fermentation provides a surplus of hydrogen and no supplemental makeup hydrogen is required for upgrading. Table 19 shows the key specifications for the fuel finishing reaction. 
Table 19. Fuel Finishing (Hydrotreating) Reaction Parameters

\begin{tabular}{lc}
\hline Parameter & Operating Condition \\
\hline Hydrotreating (HDO) (\%) & $100 \%$ \\
Operating Temperature & $270^{\circ} \mathrm{C}$ \\
Operating Pressure & $35 \mathrm{~atm}(500 \mathrm{psig})$ \\
Hydrogen: Feed Loading (molar) & 24.6 \\
WHSV & $3 \mathrm{hr}^{-1}$ \\
Catalyst Type & $3 \% \mathrm{Pt}_{\mathrm{Al}} \mathrm{O}_{3}$ \\
Catalyst Lifetime & $3 \mathrm{yrs}$ \\
\hline
\end{tabular}

\subsubsection{Cost Estimation}

For the $\mathrm{BDO}$ case, fermentation takes place in the same physical vessels as hydrolysis (sequential hydrolysis and fermentation, similar to the 2011 ethanol case) - utilizing the $1 \mathrm{MM}$-gal batch stirred tank bioreactors. However, for cost allocation purposes, the fraction of total batch time and associated volume spent in fermentation is allocated to Area 500 and vice-versa for hydrolysis allocated to Area 300. All design/cost details for the main fermentation and seed equipment are described in the 2011 ethanol design report; briefly, the large 1MM-gal fermentors, fourth and fifth seed fermentors (including cooling coils), and seed hold tank were quoted by Mueller Co. The smaller first, second, and third seed fermentors were quoted by A\&B Process Systems Corp. and are skidded units with jacketed cooling and agitation included. The lignin press following fermentation is based on the same unit as originally quoted in the 2011 ethanol report, as utilized for lignin dewatering from beer column stillage.

A filtered hydrolysate storage tank with a residence time of 20 minutes is included to provide intermediate storage for filtrate from the belt filter. The clarified BDO polishing filtration system includes two parallel skid microfiltration units, including clean-in-place (CIP) system and backwash. Installed equipment cost is $\$ 1.6 \mathrm{MM}$ total for the two skids. The ion exchange system uses a separatebed configuration, with strong acid cation and weak base anion resins. Estimated installed equipment cost is $\$ 4.5 \mathrm{MM}$ including the resins, based on scaling from cost quotations provided by Harris Group in NREL's 2015 design case (the provided cost did not break out equipment versus resin cost details separately). In total, installed capital costs for the solid separation and polishing steps are estimated at \$12.1 MM. The BDO upgrading and oligomerization reactors were both costed based on the APR reactor in the NREL 2015 catalytic upgrading design case [9], with costs scaled based on the full flow rate into the reactors. Costs for hydrotreating (hydrogenation) were set consistently with the hydrotreater utilized in the acids case (discussed below), after adjusting for more mild operating conditions in this case. All catalyst costs are summarized in Section 4.3.

Although the 2,3-BDO upgrading reactor exhibits the ability to process a high level of water, more concentrated BDO levels with less water would reduce reactor capital costs as well as heat demand to raise the temperature of the feed stream from $35^{\circ} \mathrm{C}$ to $250^{\circ} \mathrm{C}$. Compared with conventional distillation, membrane pervaporation may be an alternative option with a lower energy demand. For example, ORNL is investigating a "HiPAS" membrane system that may offer a more energy efficient, larger flux, and economical separation option for BDO concentration. Without operating/design details for this system, on a high level, the installed capital cost for this addition would need to be below \$17 MM to offer net MFSP savings through lower energy demands if $50 \%$ of the water could be removed through this operation. 
In general for the acids case, the acid fermentation units maintained the same equipment and cost basis as the BDO case (based on the 2011 ethanol design report). Fermentation vessels continue the use of 304SS to accommodate corrosive environments more pronounced with the production of carboxylic acids. The same seed train equipment/costs are also maintained consistently with the BDO case, based on the 2011 report. The pertractive membrane extractor system is based on a vendor cost estimate as well as guidance and costing for vacuum distillation for solvent recovery. The acid condensation reactor maintains a similar basis as the catalytic APR reactor design described in the 2015 catalytic design report [9].

The recovery flash, scrubber tower, and decanter costs were estimated using Aspen Capital Cost Estimator (ACCE) along with the process stream phase separation properties [103]. The ketone condensation reactors and catalyst filtration unit and solvent recycle tower were costed using ACCE. The reactors are modeled as continuous stirred tank units and account for the residence time at the modeled flow rate. The hydrodeoxygenation reactor is designed as a fixed bed hydrotreater with hydrogen mixing internals. The base reactor price quote was estimated based on prior NREL TEA studies on hydrotreater equipment capital costs and correlations to liquid feed volume flow. The base conditions for the hydrotreater (pressures $>1000$ psig and temperatures $>310^{\circ} \mathrm{C}$ ) were de-escalated to the current 500 psig operating pressure using the Guthrie pressure factor approach and the reactor size was scaled according to the total liquid volume feed entering the reactor. The hydrogen makeup compressor is a five-stage reciprocating unit, and the recycle compressor is designed as a centrifugal compressor due to the lower pressure increase in the recycle loop. Both compressor estimates include a spare unit.

\subsubsection{Achieving the Design Case}

For the BDO pathway, despite the relatively recent R\&D focus at NREL on this strategy, all key fermentation parameters have already been demonstrated within $10 \%-15 \%$ of their future targets, namely BDO process yield $(0.42 \mathrm{~g} / \mathrm{g}$ versus targets of $0.47 \mathrm{~g} / \mathrm{g})$, sugar consumption (approaching final targets for overall glucose and xylose utilization), and BDO productivity (1.7 versus $2.6 \mathrm{~g} / \mathrm{L}-\mathrm{hr}$ ). Arabinose utilization remains low to date (less than $10 \%$ converted to BDO) but this carries a much smaller cost impact than glucose and xylose, although high unconverted arabinose may increase risk for contamination and will be targeted for improvement. Similarly, performance benchmarks observed at ORNL for the BDO upgrading steps have also been demonstrated to be working well, leaving high confidence in the ability to achieve final targets by 2030. However, the largest technical hurdle that remains for this pathway is in engineering the Z. mobilis organism to eliminate the dependency on (minimal) oxygen demands for cell redox balancing, thereby making the fermentation fully anaerobic. Unlike the ethanol pathway, BDO production generates a surplus of NADH, which must be oxidized to balance cellular redox. In the presence of oxygen, the organism can oxidize NADH to NAD + using the native NADH dehydrogenase. Current experimental efforts at NREL are working on improving the redox balance by developing strategies to eliminate oxygen requirements by redirecting metabolic flux to convert excess NADH through $\mathrm{H}_{2}$ generation or introducing the NADH requiring succinate pathway (thus yielding either hydrogen or succinic acid coproducts). The former is assumed in this work.

In the event such BDO strain engineering efforts are unsuccessful, the onus would fall on the TEA modeling efforts to better understand reactor design, operation, and cost implications for microaerophilic bioreactors, which are currently a less explored space than fully anaerobic or fully aerobic fermentation. For example, recent consultation with industry has established that if low 
dissolved oxygen (DO) levels must be maintained within a strict window, this may incur similar costs and challenges as more fully aerobic fermentation (where DO uniformity would be improved by moving to smaller vessel sizes but also may be improved through increased agitation or pressure control), thus losing the advantages of anaerobic fermentation versus aerobic as described above. Initially, this was a concern for this pathway, as too little oxygen favored production of ethanol and too much favored production of a byproduct, acetoin. However, more recently ethanol coproduction has been knocked out entirely, and fermentation optimization activities have demonstrated the ability to minimize acetoin (and furthermore, catalytic upgrading activities have also demonstrated the ability to upgrade acetoin with equal performance and yields as 2,3-BDO, making any co-production of acetoin less problematic). For this reason, recent state of technology models reflecting current fermentation benchmarks have asserted the use of similar 1 MM-gal CSTR vessels as are utilized for fully anaerobic fermentation, but merely with air intake vents to allow for the presence of air/oxygen in the fermentor headspace. When coupled with potentially higher agitation mixing, this scenario is envisioned to provide sufficient DO levels in the bulk fermentation broth, even if not homogeneous throughout the full fermentor volume; this concept has been experimentally demonstrated to provide similar fermentation performance using air overlays in the fermentor headspace as air sparging into the bottom of the fermentors, albeit limited to bench-scale fermentation volumes. Moving forward, computational fluid dynamics (CFD) modeling may help to understand optimal microaerophilic bioreactor design and operation, by quantifying tradeoffs between fermentor size versus agitation power demands to provide satisfactory bulk DO concentrations.

For the acids pathway, while the majority of all key fermentation parameters have similarly been shown to be performing well relative to future targets (i.e., productivity of $1.1 \mathrm{~g} / \mathrm{L}-\mathrm{hr}$ versus $2.0 \mathrm{~g} / \mathrm{L}-\mathrm{hr}$ targets with process yields nearly at target levels), one of the largest uncertainties in the current design is the continuous separation strategy to recover the acid from the fermentation broth via pertractive membranes. Such operations could suffer from a number of potential processing failures including toxicity due to failure to control the $\mathrm{pH}$ from inefficient acid removal, potential fermentation contamination due to solvent back-filtration across the membrane into the fermentor broth, or plugging of membrane filters, to name a few. To date, acid recovery has been demonstrated to be high over short-term trials using clean substrates (including nearly 100\% recovery of butyric acid specifically), but encounters more challenges using real hydrolysate substrate broth. Over a longer timeframe, the continuous recovery and recycle of the solvents may encounter challenges if other acids or molecules extract and build up in the solvent loop, which would require periodic replacement of one or both solvents. Longer-term implications on membrane lifetimes, fouling, pump-around throughput rates, etc. are not known at this time, and will require further study in the future as more granular operational and design details are established for this system. While $\mathrm{pH}$-controlled fermentation to maintain acids as salt complexes at elevated $\mathrm{pH}$ (followed by ion exchange and neutralization) can be shown to work well experimentally, the associated costs for acid and base addition, as well as subsequent salt disposal, would be untenable.

A potential mitigation strategy is being explored in the DOE BETO-funded Bioenergy Separations Consortium. For this approach, the fermentation is controlled at a neutral $\mathrm{pH}$ via the introduction of a caustic solution and the separation strategy uses a resin wafer based electrodeionization operation that separates the conjugated base. The operation targets a nearly complete recovery and separation of the acid and base. The carboxylic acid product is sent on for further upgrading as outlined in the above design. The base is recycled to the fermentation reactor, limiting any caustic make-up requirement. The key factors that will make such an operation economically viable and align with sustainability 
goals of the integrated biorefinery hinge not only on high recovery of both the acid and the base but also the reduction of electricity demand. Recent work by Argonne National Laboratory under the Bioenergy Separations Consortium has demonstrated the reduction of both capital cost and electricity demand compared to many documented electrodialysis systems[104, 105].

Acid upgrading work has demonstrated the complete three-step conversion to a final fuel product (including initial fuel testing data), although these results have not been tested on acid extracted DMR fermentation broth and remain unoptimized in that regard. While there are additional challenges associated with an extracted catalytic acid feedstock compared to a pure substrate (e.g., minor broth impurities and entrained water), discussions with researchers point to clear mechanisms for attenuating these effects. The acid condensation reaction catalyst can be broadly tuned for acidity and is expected to tolerate up to $10 \%$ water; furthermore, because the catalyst lacks exotic metal atoms, poisoning is not expected to be an issue. After the initial catalytic step, the scrubber and phase separation of the intermediate separation scheme will preclude further downstream issues associated with impurities. Using clean butyric acid, current demonstrated yields for the acid condensation step are near $100 \%$ with very high selectivity in excess of $90 \%$ to the desired 4-heptanone product. Ketone condensation proceeds with high selectivity to the desired enone product under the controlled lower temperature conditions with toluene as a solvent, with the current focus on reducing the residence time to the 15hour target or less.

Current work is also exploring a solventless "neat" configuration (i.e., greatly reduced capital costs) but suffers from low conversion and poor selectivity to the desired fuel molecule to date. Similarly, a high-temperature approach achieving near complete conversion is being explored but causes excessive condensation to heavier less desirable fuel products. The final hydrotreating step is generally considered established technology and not currently a focus of NREL R\&D. In the future, as upstream conversions improve, renewed focus on HDO may find further improvements or updated targets that improve the overall economics, such as platinum loading or WHSV.

\subsection{Area 600: Wastewater Treatment}

\subsubsection{Overview}

Wastewater is generated in the process from condensed pretreatment flash vapor in Area 200, the fermentation broths in Area 500 and 700 (after separation of product and cells), and minor sources such as boiler and cooling tower blowdown. All such wastewater is sent to the WWT system in Area 600. After treatment, the effluent water is assumed clean and fully reusable by the process, reducing both the fresh makeup water requirement and discharge to the environment.

In previous design reports, the chemical oxygen demand (COD) loading to wastewater treatment was relatively large due to high concentrations of soluble unconverted biomass components, primarily extractives. These components ultimately arrived at WWT in the stillage or aqueous phase postfermentation, or in the black liquor produced in deacetylation. NREL consulted with Brown and Caldwell in 2010 and again in 2012 to design treatment schemes that included anaerobic digestion of $80 \%$ of this organic material to biogas, followed by aerobic digestion of the remaining organics and final cleanup by reverse osmosis. The biogas was used as boiler fuel for heat and power, and the reverse osmosis (RO)-treated water was recycled to the process. 
In the present design, the black liquor containing the extractives as well as soluble lignin is combined with further deconstructed solid lignin and sent to aerobic fermentation with Pseudomonas putida in Area 700. Given the scavenging nature of P. putida, we reasoned that these extractives components would likely be consumed in the aerobic fermentation, forming cell mass and $\mathrm{CO}_{2}$ as well as some organic acids, as dictated by the organism's engineered metabolism (discussed in the next section). Given the elimination of extractives, and drastic reduction in other organics solubilized to DMR black liquor (i.e., carbohydrates and solubilized lignin) that ultimately reach WWT given the addition of the Area 700 train, COD loading in the WWT feed stream is reduced by about $92 \%$ (acids case) and $82 \%$ (BDO case, given lower overall sugar utilizations) relative to the 2013 design report ( 9 and $21 \mathrm{~g} / \mathrm{L}$ respectively versus $120 \mathrm{~g} / \mathrm{L}$ in the 2013 design), obviating the anaerobic digestion requirement. The present design therefore begins at aerobic digestion; the updated simplified flow diagram is shown in Figure 12.

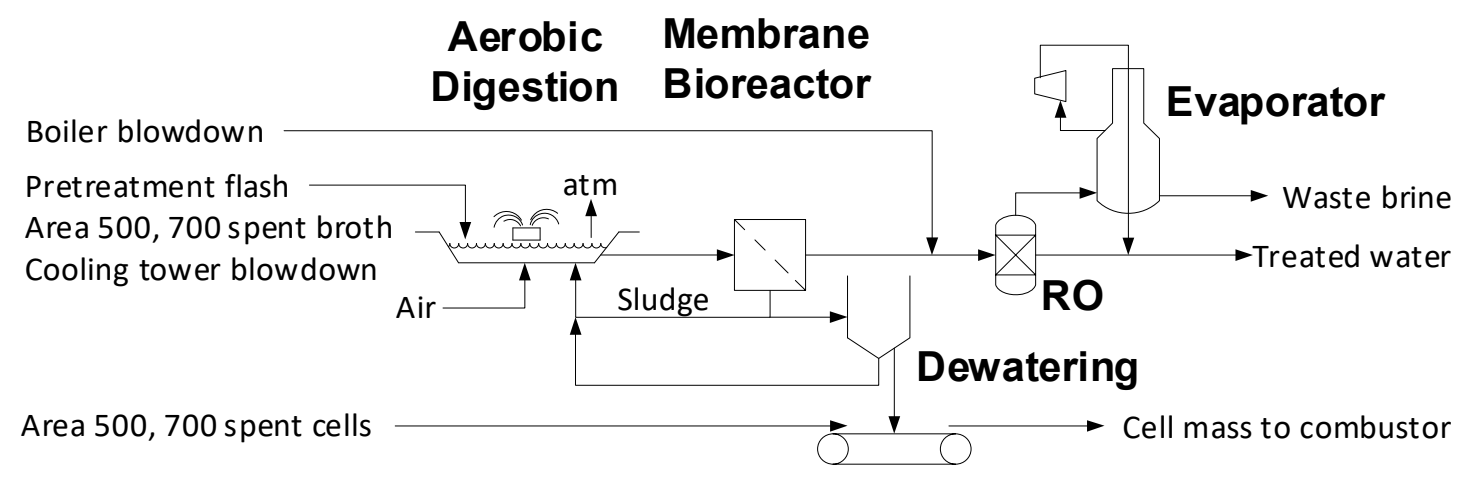

Figure 12. Simplified flow diagram of the WWT process

The aerobic system comprises several large basins or ponds that are aerated from the bottom via a grid sparger. The water in the basins is continuously cycled through membrane bioreactors, which are aeration tanks with ultrafiltration membranes that yield a clean water stream and a low-solids biomass sludge stream, which is mostly recycled to the basin. The wasted fraction of sludge that is not recycled passes first through a gravity belt thickener, then is subsequently centrifuged to $>20 \%$ solids. The fermentation cell mass separated in Areas 500 and 700 is collected along with the digester sludge, and the combined solid mass is routed to the boiler. The water effluent of the membrane system is sufficiently clean to feed directly to RO to remove dissolved salts. The RO permeate is recycled to the process and the RO retentate is concentrated in an evaporator to produce a brine, sold in this design as a co-product.

\subsubsection{Design Basis}

The present WWT system follows the design basis from the 2012 Brown and Caldwell subcontract, as detailed in the 2013 design report, from aerobic digestion forward. In the Brown and Caldwell design, the anaerobic system removed $80 \%$ of the influent COD, transferring $2.67 \mathrm{MM}$ gal $/ \mathrm{d}$ of water to aerobic treatment at $13.3 \mathrm{~g} / \mathrm{L}$ COD. For the acids case, the hydraulic load to aerobic treatment is larger than this, but COD loading is lower. For the BDO case, the hydraulic load is smaller but COD load higher. Nitrogen loading in both cases is significantly lower than assumed in the Brown and Caldwell design - this indicates that supplemental ammonia will be required to support culture growth. The relevant design parameters of the wastewater system are summarized in Table 20. 
Table 20. WWT System Design Basis

\begin{tabular}{lccc}
\hline $\begin{array}{l}\text { Aerobic digestion influent } \\
\text { conditions }\end{array}$ & $\begin{array}{c}2012 \text { Brown and } \\
\text { Caldwell design (used } \\
\text { for scaling) }\end{array}$ & $\begin{array}{c}\text { This design (acids } \\
\text { case) }\end{array}$ & $\begin{array}{c}\text { This design (BDO } \\
\text { case) }\end{array}$ \\
\hline Hydraulic load & $2.7 \mathrm{MM} \mathrm{gal} / \mathrm{d}$ & $2.8 \mathrm{MM} \mathrm{gal} / \mathrm{d}$ & $2.2 \mathrm{MM} \mathrm{gal} / \mathrm{d}$ \\
Total COD & $13.3 \mathrm{~g} / \mathrm{L}$ & $9.3 \mathrm{~g} / \mathrm{L}$ & $21 \mathrm{~g} / \mathrm{L}$ \\
Total Kjeldahl N & $724 \mathrm{mg} / \mathrm{L}$ & $66 \mathrm{mg} / \mathrm{L}$ & $38 \mathrm{mg} / \mathrm{L}$ \\
\hline
\end{tabular}

The wastewater feed is pumped to the aerobic activated-sludge basin. The basin construction is concrete and steel. Air is delivered through a submerged grid sparger; surface aerators are also specified to enhance evaporative cooling during summer months. In aerobic digestion, $96 \%$ of the soluble organic matter is removed, with $74 \%$ producing water and carbon dioxide and $22 \%$ forming cell mass. The contents of the pond are cycled continuously though membrane bioreactors for clarification.

The membrane retentate is a low-solids sludge stream that is mostly recycled back to the basin. A fraction of the sludge is removed from the loop and pumped to a dewatering system. Brown and Caldwell specified gravity belt thickeners to first concentrate the sludge to $4 \%$ solids, followed by centrifuges with $95 \%$ solids capture rate and a cake concentration of $20 \%$ solids. The centrate is recycled with polymer addition to the aerobic basins. Dewatered sludge is then conveyed to the combustor in A800. The treated water is pumped to a RO membrane system for salt removal. RO produces a brine containing primarily sodium sulfate along with all remaining ions and organics. The brine is further concentrated in a mechanical-vapor-recompression evaporator to $50 \%$ solids and the condensate is also recycled to the process. Finally, the brine is further processed through a dryer and centrifuge to crystallize the salt for sale as a coproduct. This was deemed necessary in the present model configuration in light of the substantial quantities of caustic (sodium hydroxide) and sulfuric acid used in multiple points throughout the facility, which would incur unreasonable costs without either recovering those input chemicals or else offsetting a portion of the chemical costs by selling the sodium sulfate salt. The latter is elected here given that the salt is already produced at high purity as a byproduct from wastewater treatment (over $98 \%$ purity of the brine solids).

\subsubsection{Cost Estimation}

Brown and Caldwell estimated the capital costs of the wastewater system components, drawing on support from technology vendors. In previous design reports, the individual unit costs were scaled to either the hydraulic flow rate or COD load to anaerobic digestion. For the present model, with anaerobic digestion removed, the remaining components (aerobic digestion, sludge dewatering, reverse osmosis, evaporation, and centrifugation) were re-baselined to the total flow and COD loading of the stream leaving anaerobic digestion in Brown and Caldwell's design (2.67 MM gal/d at 13.3 g/L COD) and scaled from there. Additional equipment costs for sodium sulfate purification were scaled from a recent subcontract with Nexant for an evaporation flash drum, dryer, and centrifuge designed for this purpose. 
Table 21. Installed Capital Costs for Area 600

\begin{tabular}{lcc}
\hline Equipment & Acids case & BDO case \\
\hline Aeration basins & $\$ 9.5 \mathrm{MM}$ & $\$ 8.1 \mathrm{MM}$ \\
Membrane bioreactor & $\$ 7.8 \mathrm{MM}$ & $\$ 6.0 \mathrm{MM}$ \\
Reverse osmosis system & $\$ 4.2 \mathrm{MM}$ & $\$ 3.2 \mathrm{MM}$ \\
Dewatering & $\$ 2.4 \mathrm{MM}$ & $\$ 3.3 \mathrm{MM}$ \\
Others (pumps, conveyer, etc.) & $\$ 3.5 \mathrm{MM}$ & $\$ 4.8 \mathrm{MM}$ \\
Brine evaporator & $\$ 7.7 \mathrm{MM}$ & $\$ 6.6 \mathrm{MM}$ \\
Sodium sulfate purification & $\$ 3.9 \mathrm{MM}$ & $\$ 3.6 \mathrm{MM}$ \\
Totals & $\$ 38.8 \mathrm{MM}$ & $\$ 35.6 \mathrm{MM}$ \\
\hline
\end{tabular}

\subsection{Area 700: Lignin Upgrading}

\subsubsection{Overview}

This process area covers the key unit operations for deconstruction and conversion of lignin (and other residual biomass components) to coproducts, as a critical element to enabling the $\$ 2.50 / \mathrm{GGE}$ MFSP targets for biochemical processing. In summary, the black liquor product from DMR pretreatment is combined with the residual solids stream separated during $\mathrm{CEH}$ (acids case) or from fermentor broth clarification (BDO case) and routed to a more severe "base catalyzed deconstruction" (BCD) unit employing a caustic soaking process at elevated temperature to further deconstruct high-molecular weight lignin to metabolically accessible compounds. The caustic from DMR pretreatment that exits with the black liquor offsets a large portion of the caustic required to achieve the elevated $\mathrm{pH}$ in the BCD step. The soluble liquid BCD product is then routed to bioconversion, which converts soluble lignin and other residual organics to muconic acid through an aerobic $\mathrm{pH}$-controlled bioconversion with $P$. putida. The product is initially produced as a muconate salt at the given fermentation $\mathrm{pH}$ (i.e. the caustic from DMR/BCD neutralizes muconic acid to maintain near-neutral $\mathrm{pH}$ forming sodium muconate), which is then isolated in high purity as muconic acid crystals following acid addition and low-pH crystallization. The muconic acid crystal is redissolved in a carrier solvent then hydrogenated under mild conditions to adipic acid, which is subsequently recovered through another crystallizer. A schematic diagram for the process is shown in Figure 13.

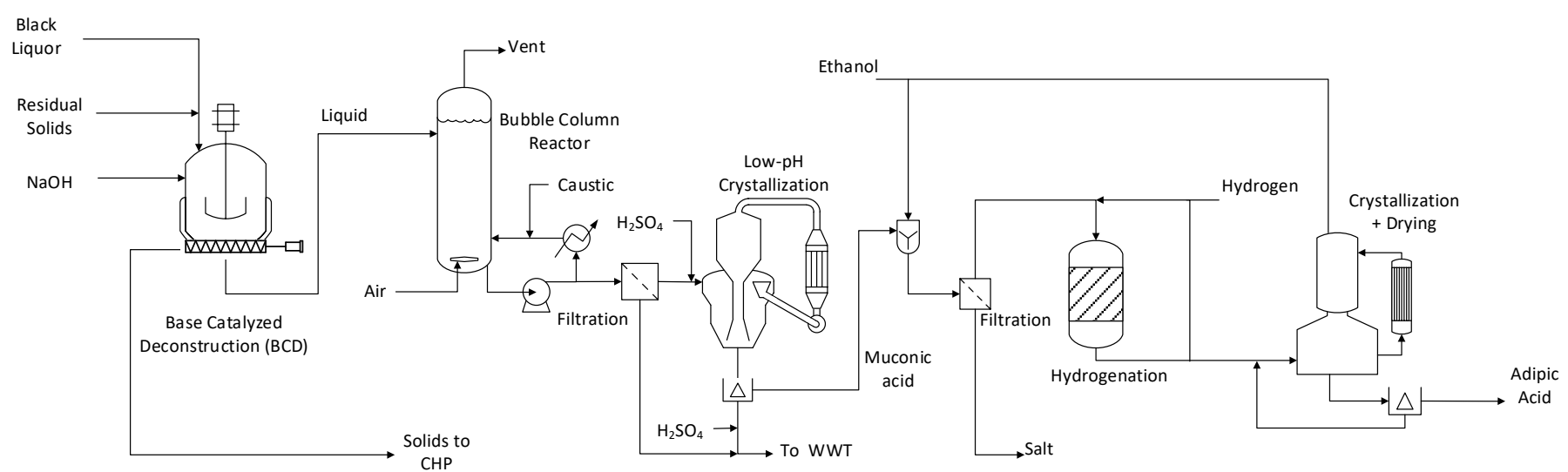

Figure 13. Schematic diagram of lignin deconstruction and conversion process to coproducts 
Historically, in prior biochemical designs residual lignin and solids were burned using a high-solids biomass boiler to produce steam and power for the process, which realizes minimal additional economic value to the carbon present in the lignin but does offset power and natural gas (NG) imports which substantially improve the sustainability of the integrated process design. A second option is to upgrade a fraction of the available lignin/unconverted carbon to a value-added coproduct. The 2013 biochemical design report presented an initial analysis around lignin utilization to coproducts, ultimately concluding that nonoxygenated lignin coproducts (e.g., cyclohexane) provide limited opportunity for achieving $\$ 3 / \mathrm{GGE}$ or lower cost targets, as well as showed an increase in overall greenhouse gas emissions when compared to the baseline design of burning lignin for heat and power. Other product options with higher atom efficiencies that produced oxygenated value-added chemicals were predicted to be viable for meeting such MFSP targets and improved overall sustainability of the integrated design, even when requiring power and natural gas imports.

In light of these early conclusions, research shifted to production of oxygenated intermediates that take advantage of the underlying structure of the biomass composition. While a wide array of coproducts are possible from lignin [106, 107], in this design, adipic acid is highlighted as a representative example coproduct from lignin. Adipic acid is an oxygenated di-carboxylic acid coproduct primarily utilized to produce nylon and other polymeric materials [11]. Not only is the market value of adipic acid ideal for meeting out-year $\$ 2.50 / \mathrm{GGE}$ cost goals at roughly $\$ 0.86 / 1 \mathrm{~b}$, but the direct replacement of adipic acid via the chosen process improves sustainability compared to traditional petroleumderived adipic acid produced in a two-stage process that involves energy-intensive oxidation of cyclohexane followed by nitric acid oxidation of the intermediate to adipic acid [10]. The harsh latter steps of the synthesis have a large impact on the sustainability of the fossil-based process, which are absent in the current biological route [108]. Additionally, adipic acid possesses a large market volume over 2.3 MM tonne per year globally[11](roughly 2.8 MM tonne/year currently), thus exceeding smaller "niche" market products that may enable high values but not at volumes that could sustain multiple biorefineries at commodity scale.

Valorization of residual carbon in the lignin rich solids requires three conceptual steps: deconstruction, conversion, and upgrading. During deconstruction, recalcitrant solids are subjected to chemical treatment to produce a more tractable material for subsequent conversion. During this step, the goal is to reduce the molecular weight of the substrate to promote solubilization and increase the amount of accessible chemical compounds entering the conversion stage. Alkaline pretreatment using a strong base increases the lignin solubilization owing to the deprotonation of the hydroxyl moieties of the macromolecule, and initiates cleavage of beta-O-4 bonds generating more accessible lignin monomers, dimers, and oligomers.

After deconstruction, the material is sent to the conversion phase, wherein the accessible carbon is fermented to a single desired product intermediate. Compared to catalytic approaches operating on complex mixtures that suffer from poor selectivity to any single product but high conversion of all substrates (e.g., pyrolysis oils and hydrothermal liquefaction), using a biological approach provides the opportunity for high selectivity to a single product and combines what otherwise requires multiple successive reaction and fractionation units. This high specificity is critical as many coproduct chemicals require high homogeneity and purity (particularly for products in technical markets such as polymers or nutritional supplements). 
To achieve these desired outcomes and channel a diverse mixture of carbon compounds to a single desired product requires a strain capable of two major behaviors. First, a robust and diverse set of catabolic pathways covering acids, sugars, and aromatics is necessary for high conversion. Second, the ability to engineer a heterologous pathway or pathway(s) connecting the consumed substrates to a single product is necessary for high selectivity to a single product. Both of these phenomena are readily accomplished in isolation (e.g., mixed cultures often can exhibit high conversion of complex mixtures; highly engineered single strains show high selectivity to products from individual substrates). However, combining the two behaviors is challenging due to the frequently competing goals of the host regulatory network in catabolic strains and the lack of catabolic diversity in standard engineered hosts that do possess selective pathways. More recent work focused on expanding the industrially relevant host strains past the familiar E. coli and $S$. cerevisiae has enabled the rapid onboarding of a more optimal funneling host, $P$. putida. This host displays both desired behaviors, and importantly has proven to be genetically tractable to metabolic engineering strategies.

The final step following conversion is recovery and upgrading to the desired coproduct. Recent work has shown the successful use of temperature and $\mathrm{pH}$ to crystallize dicarboxylic acids (i.e., muconic acid and its downstream derivative adipic acid) from aqueous solution using the differing temperature solubility curves of the protonated/deprotonated species.

\subsubsection{Design Basis}

The lignin utilization process was designed based on existing literature, ongoing NREL research, and communications with select contractors in the field. Black liquor exiting the counter-current deacetylation reactor is combined with the residual solids exiting enzymatic hydrolysis (lignin press on the whole slurry material in the BDO case, and on the residual solids stream exiting $\mathrm{CEH}$ in the acids case). The residual solids consisting of $\sim 70 \%$ lignin by weight enters at near ambient temperature as a $\sim 40 \%$ solid paste/slurry.

As noted earlier in the report, the black liquor contains acetate, extractives, sucrose, carbohydrates, and nearly half of the original lignin present in the biomass (of which $15 \%$ is already in monomer form) as a low $\mathrm{pH}$ aqueous solution. This black liquor solution is routed directly from the reactor at $90^{\circ} \mathrm{C}$ and mixed with the downstream residual solids slurry, which reduces heating requirements for the subsequent deconstruction step. Combined, this mixture contains $99 \%$ of the lignin originally present in the biomass as a complex slurry of solids, solubilized solids, and soluble components. Table 22 shows the composition of the two feed streams.

Table 22. Composition of Both Lignin Streams in Model

\begin{tabular}{lcc}
\hline Parameter & Black Liquor & Residual Solids \\
\hline Total Solids ${ }^{a}$ (wt \%) & $12.5 \%$ & $63.1 \%$ \\
Species Distribution (\% total solids) & & \\
Lignin & $52.9 \%$ & $48.7 \%$ \\
Cellulose & $5.0 \%$ & $7.4 \%$ \\
Xylan & $13.9 \%$ & $1.2 \%$ \\
Arabinan & $5.1 \%$ & $0.1 \%$ \\
Other Solids (ash, protein, etc.) & $23.1 \%$ & $42.6 \%$ \\
\hline a Total solids defined as lignin, soluble lignin, cellulose, xylan, arabinan, manan, galactan, ash, and protein.
\end{tabular}


The combined stream is routed to a BCD reactor operating at elevated temperatures to further deconstruct the lignin and solubilized oligomers. Caustic is added to achieve a minimum of $2 \mathrm{wt} \%$ loading based on the feed mixture. In the current design, due to the higher caustic loading and more concentrated black liquor accompanying the counter current configuration, the combined mixture has a caustic loading of $5.1 \%$, in excess of the $2 \%$ threshold and no additional caustic is needed. The treated mixture is pressurized to $6.3 \mathrm{~atm}$ to prevent vaporization throughout the duration of the reaction, then reacted for 30 minutes at a temperature of $120-160^{\circ} \mathrm{C}$. At present the model assumes a basis of $120^{\circ} \mathrm{C}$ based on latest experimental conditions, but research is ongoing to identify the most optimal temperature for this operation, and may be increased to as high as $160{ }^{\circ} \mathrm{C}$ if this is found to promote better solubilization/deconstruction to usable components. This step represents a key cost driver to the overall process, as evidenced in the "metabolically accessible lignin" parameter in the Sensitivity Analysis below (Section 5.2), and will require continued R\&D focus to better understand and optimize moving forward.

Based on experimental work done at NREL, the more severe temperatures promote higher solubilization of the lignin solids, although there is a balance as increased severity eventually degrades the solids to a mixture of short chain acids and alcohol products. In the current design, $36 \mathrm{wt} \%$ of the solids entering the BCD reactor are solubilized to metabolically accessible monomers/oligomers. Of the solids present, this includes $48 \mathrm{wt} \%$ solubilization of carbohydrates and $53 \mathrm{wt} \%$ of the lignin. The mixture stream from this step is fractionated with the residual solids sent to the boiler, while the solubilized product is cooled and then routed to biological funneling. Table 23 summarizes the BCD reaction conditions and conversions used in the current design.

Table 23. Reaction Conditions and Key Parameters for Lignin BCD

\begin{tabular}{lc}
\hline Parameter & Black Liquor \\
\hline Temperature, ${ }^{\circ} \mathrm{C}$ & 120 \\
Pressure, atm & 6.32 \\
NaOH loading (minimum required), wt $\%$ & $5.1 \%(2 \%)$ \\
Residence time, min & $30 \mathrm{~min}$ \\
Total solids $^{\mathrm{a}}$, wt $\%$ & $22.3 \%$ \\
Total solubilization, wt \% solids $^{\circ}$ & $36 \%$ \\
Deconstruction extents & \\
Cellulose & $48 \%$ \\
Xylan & $48 \%$ \\
Arabinan & $48 \%$ \\
Lignin & $53 \%$ \\
solids defined as lignin, soluble lignin, cellulose, xylan, arabinan, mannan, galactan, ash, and protein.
\end{tabular}

Biological funneling occurs through an engineered strain of $P$. putida KT2440, a naturally occurring gram-negative soil bacterium. This strain of Pseudomonas is a variant of strains isolated and heavily studied since the 1960s for their novel capability to catabolize aromatics via the ß-ketoadipate pathway [109-111]. In 2002, the first complete genome was published and expanded the understanding of the aromatic catabolic potential, reigniting interest in its bioremediation applications [112]. Subsequent annotation of the genome pointed to an even more expansive biological toolset including pathways for over 92 compounds (spanning carbon, nitrogen, and phosphorous sources in the natural environment) and physiological mechanisms improving growth under high $\mathrm{pH}$, osmolarity, and the ability to carry strongly oxidative reactions [113]. Work at NREL over the past 4 years focusing on multiple aspects 
of the genomic capabilities has led to significant steps forward in the required funneling behavior $[114,115]$.

In addition to funneling, $P$. putida work at NREL is focused on achieving high selectivity to an adipic acid coproduct. Public literature and patents indicate multiple proposed/known routes to bio-adipic acid from lignin compounds via muconic acid, ß-ketoadipate, or direct production using an enolase for a final metabolic reduction reaction [116-120]. For this design, aerobic production of muconic acid was selected, which avoids the difficult final reduction reaction, but also has benefits for separations as outlined in further detail below. Specifically, metabolic engineering was used to generate a recombinant strain of KT2440 that prevents lignin catabolic intermediates from entering the central carbon metabolism stopping at cis-cis-muconic acid [121-123]. Additionally, sugar metabolism was modified to divert carbon away from central carbon metabolism and into the upper ß-ketoadipate pathway, providing a mechanism to valorize multiple substrates to the desired coproduct [121]. Overall, the modeled strain funnels lignin and sugars, as well as long chain acid substrates (e.g., extractives), to muconic acid, with shorter acid species (e.g., acetate) contributing exclusively to growth [124]. The combined effect is to partially decouple growth from production leading to greater overall carbon retention to coproducts than previous attempts. The maximum theoretical metabolic yield of muconic acid as well as product diversion for each substrate were estimated using flux balance analysis on the metabolic reconstruction for the designed strain $[125,126]$. The theoretical maximum yield of muconic acid from sugars was estimated as $0.58 \mathrm{~g} / \mathrm{g}$ glucose and $0.59 \mathrm{~g} / \mathrm{g}$ xylose.

As noted in Section 2.1, the "extractives" component in the delivered biomass feedstock represents a significant fraction of the overall biomass, roughly $15 \mathrm{wt} \%$, compared to the lignin fraction at $16 \mathrm{wt} \%$. Although a portion of this component reflects compositional mass balance closures below 100\% (representing unknown or unmeasured components), the remainder of the extractives fraction is generally made up of simple components such as sugars, sugar alcohols, and sugar acids, and thus would be readily consumed by $P$. putida. However, given that this can be such a variable component both in the amount and type of extractives present in the delivered biomass feedstock (depending on parameters such as harvest location, time, biomass type, and storage/preprocessing logistics), we did not want to over-credit the TEA by converting all of it to coproduct, nor over-penalize the TEA by ignoring it through the lignin train for it to then add significant COD loading to the wastewater treatment section downstream. Thus, given that whatever level of extractives do make it to the facility will be metabolized by P. putida, the model treats "extractives" as a long chain acid species, gluconic acid. In the event that extractives could not be utilized at high levels in parallel with solubilized lignin, muconic acid yields would be reduced and residual COD loading to wastewater treatment would be higher (potentially necessitating a move back to including AD in the WWT section as well). In such a case, future plans will investigate other processing alternatives to maintain the \$2.50/GGE MFSP goals including biogas utilization, alternative lignin valorization approaches, and other strategies as outlined at the end of this report in Section 5.4.

The current bioconversion area begins by diluting a subset of the hypertonic BCD solubilized product stream approximately twofold for use in the seed reactors, as well as prefill cycles of production as needed to reduce any osmotic shock experienced by the fermentation organism. Additionally, this attenuates potential toxicity issues with an overly concentrated aromatic stream that are the topic of ongoing research [127]. After this mild conditioning, the feed is cooled to $32^{\circ} \mathrm{C}$ and a portion of the feed stream is diverted for use in the seed train, where inoculum is grown and concurrently acclimated to the harsher conditions of this fermentation. The seed train uses three stages and begins with a small 
$0.3 \mathrm{~m}^{3}$ reactor inoculated from the lab, followed by a $1: 10$ increase to $3 \mathrm{~m}^{3}$. Because an aerobic fermentation enables more rapid growth as well as dense cell cultures compared to an equivalent anaerobic system, a larger $100 \mathrm{~m}^{3}$ third stage with a constant diameter of $2.8 \mathrm{~m}$ and a height of $16.8 \mathrm{~m}$ or roughly $1 / 10^{\text {th }}$ the size of the production reactor is used for the final inoculum preparation prior to transferring into the production reactor [128].

Nutrients during the seed reactions include DAP for phosphorus supplementation and host specific nutrients required to properly acclimate the strain to the final feed stream with an assumed cell elemental composition of $\mathrm{C}_{1} \mathrm{H}_{1.8} \mathrm{O}_{0.5} \mathrm{~N}_{0.2}$ accounting for the higher intracellular amounts of protein utilized during exponential growth (and associated nitrogen content). The current model assumes only sugars (presumed preferential substrate) are consumed during the seed phases. Table 24 shows the conversions and reactions used in the seed fermentation.

Table 24. Stoichiometry and Conversions Applied for Lignin Bioconversion Seed Train

\begin{tabular}{llc}
\hline Reaction & Reactant & $\begin{array}{c}\text { \% Converted to } \\
\text { product }\end{array}$ \\
\hline Seed Train: & & \\
Glucose $+0.047 \mathrm{CSL}^{\mathrm{a}}+0.018 \mathrm{DAP} \rightarrow 6$ P. putidagrow $+2.4 \mathrm{H}_{2} \mathrm{O}$ & Glucose & $46 \%$ \\
Glucose $+1.94 \mathrm{O}_{2} \rightarrow 0.74$ Muconic $+1.57 \mathrm{CO}_{2}+3.78 \mathrm{H} 2 \mathrm{O}$ & Glucose & $54 \%$ \\
Xylose $+0.039 \mathrm{CSL}+0.015 \mathrm{DAP} \rightarrow 5 P$. putidagrow $+2 \mathrm{H}_{2} \mathrm{O}$ & Xylose & $46 \%$ \\
Xylose $+1.57 \mathrm{O}_{2} \rightarrow 0.62 \mathrm{Muconic}+1.26 \mathrm{CO}_{2}+3.13 \mathrm{H} 2 \mathrm{O}$ & Xylose & $54 \%$ \\
Arabinose $+0.039 \mathrm{CSL}+0.015 \mathrm{DAP} \rightarrow 5 \mathrm{P}$. putidagrow $+2 \mathrm{H}_{2} \mathrm{O}$ & Arabinose & $46 \%$ \\
Arabinose $+1.57 \mathrm{O}_{2} \rightarrow 0.62$ Muconic $+1.26 \mathrm{CO}_{2}+3.13 \mathrm{H} 2 \mathrm{O}$ & Arabinose & $54 \%$ \\
\hline
\end{tabular}

${ }^{\mathrm{a}} \mathrm{CSL}$ and DAP are both nitrogen sources required for growth. The stoichiometry shown above is only used to balance the compositions assumed for cell mass.

Building on extensive studies and experience with aerobic fermentation reactor modeling developed during prior work and bench-scale runs supporting aerobic lipid production (detailed previously in section A500), the remaining feed is sent to supply large production reaction bubble columns shown in Figure 14.

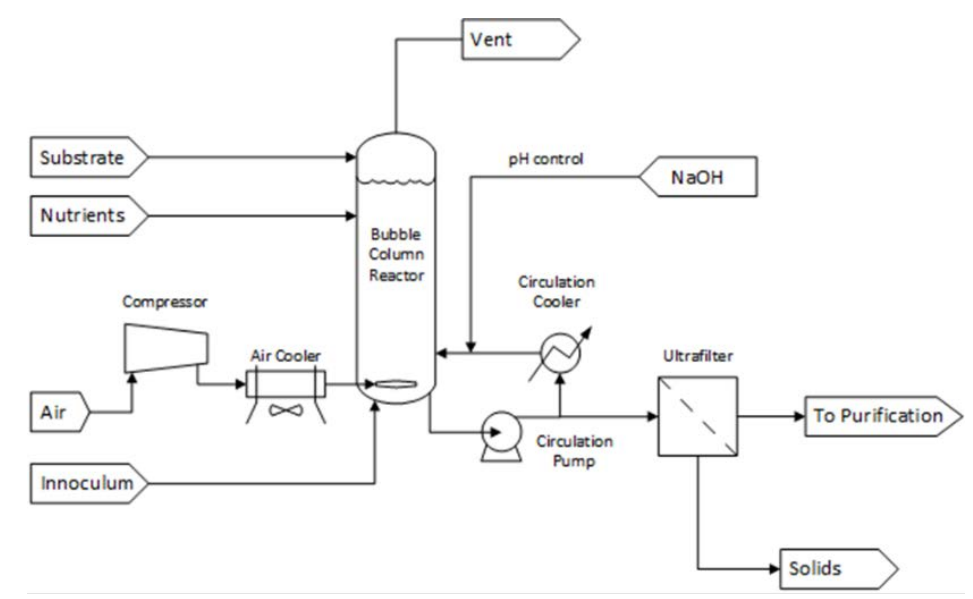

Figure 14. Aerobic bubble column bioreactor setup 
A pressurized air stream is sent to a sparger at the base of the column. As the gas travels upwards bubble expansion and hydrodynamics promote mixing and mass transfer throughout the two-phase system. Compared to stirred tank reactors that use motor speed to achieve sufficient mixing and control the sparge rate to achieve sufficient gas transfer, bubble columns only provide a single control, the gas sparge rate, to achieve both mixing and mass transfer. Despite this drawback in flexibility, at larger volumes bubble columns provide more cost-efficient oxygen delivery as well as reduced shear on the organism due to absence of mechanical components [13]. Additionally, the simpler mechanical design aids in aseptic operation. To date, the TEA models assume standard bubble column designs outfitted with air sparge rings at the bottom of the columns; however, more advanced designs may be possible to aid in improving oxygen mass transfer at lower aeration rates (such as advanced baffle designs, microfine bubble sparging, air input port placement, etc.), which may be considered in the future. Recent NREL work has begun to investigate such implications across different design configurations through computational fluid dynamics (CFD) modeling, which may be leveraged in the future to further optimize aerobic bubble column design.

To meet the higher heat demands associated with aerobic fermentation, each column is outfitted with a pump-around cooling loop running through a chilled-water heat exchanger. Previous design reports indicated the importance and outlined in greater detail the traditional equations used to estimate mass transfer in stirred tank reactors. In the current model, a correlation relating the superficial velocity, temperature, and viscosity of the fluid to the overall mass transfer coefficient in a bubble column is used in combination with Henry's law and the oxygen partial pressure of the gas at the top and bottom of the bubble column to explicitly determine the OTR [129]. The necessary amount of pressurized air delivered to the system is then determined such that the OTR is equivalent to the OUR, assuming all oxygen delivered to the liquid phase is available to the organism without transport limitations. Equation 1 shows the correlation and oxygen transport rate equations used in the model [129]:

$$
\begin{aligned}
& k_{L} a=0.32\left(u_{S}\right)^{0.7}\left(\mu_{e f f}\right)^{-0.84} \times 1.025^{(T-20)} \\
& \text { OUR }=\text { OTR }=k_{L} a\left(C^{*}-C_{L}\right)_{\text {MEAN }}
\end{aligned}
$$

Nutrients supplied during the seed reactions include DAP for phosphorus supplementation and ammonia to provide sufficient nitrogen to the strain. The production reaction occurs over a longer period (not exclusively focused on growth and acclimation) and the cell is expected to enter a stationary phase. To account for the slower specific growth rate and lower nitrogen demand accompanying stationary phase the cell elemental composition is assumed to be $\mathrm{C}_{1} \mathrm{H}_{1.85} \mathrm{O}_{0.828} \mathrm{~N}_{0.058}$ during the production reaction. The net metabolic stoichiometry and conversion of the muconic acid fermentation is a combination of theoretical maximum metabolic yields and maintenance/respiration reactions dependent on the specific substrate consumed. While it is likely regulatory effects will initially cause a substrate diauxic preference, where one carbon source may be consumed prior to others, it is assumed the target strain will be sufficiently engineered to co-consume $98 \%$ of the carbon sources over the course of a fill/draw cycle and avoid large carbon losses to the exiting broth. For the production reaction, Table 25 lists the biological reactions and conversion to product for the carbon sources in the feed stream. 
Table 25. Stoichiometry and Key Conversions for Bioconversion of Lignin and Residuals

\begin{tabular}{|c|c|c|}
\hline Reaction & Reactant & $\%$ Diverted to product \\
\hline \multicolumn{3}{|l|}{ Production Reactor: } \\
\hline Glucose $+1.18 \mathrm{O} 2+0.28 \mathrm{NH}_{3} \rightarrow 4.8$ P. putida $+1.2 \mathrm{CO}_{2}+1.98 \mathrm{H} 2 \mathrm{O}$ & Glucose & $46 \%$ \\
\hline Glucose $+1.94 \mathrm{O}_{2} \rightarrow 0.74$ Muconic $+1.57 \mathrm{CO}_{2}+3.78 \mathrm{H} 2 \mathrm{O}$ & Glucose & $54 \%$ \\
\hline Xylose $+0.98 \mathrm{O} 2+0.23 \mathrm{NH}_{3} \rightarrow 4$ P. putida $+1 \mathrm{CO}_{2}+1.64 \mathrm{H} 2 \mathrm{O}$ & Xylose & $46 \%$ \\
\hline Xylose + $1.57 \mathrm{O}_{2} \rightarrow 0.62$ Muconic + $1.26 \mathrm{CO}_{2}+3.13 \mathrm{H} 2 \mathrm{O}$ & Xylose & $54 \%$ \\
\hline Arabinose $+0.98 \mathrm{O} 2+0.23 \mathrm{NH}_{3} \rightarrow 4$ P. putida $+1 \mathrm{CO}_{2}+1.64 \mathrm{H} 2 \mathrm{O}$ & Arabinose & $46 \%$ \\
\hline Arabinose $+1.57 \mathrm{O}_{2} \rightarrow 0.62$ Muconic $+1.26 \mathrm{CO}_{2}+3.13 \mathrm{H} 2 \mathrm{O}$ & Arabinose & $54 \%$ \\
\hline Sucrose $+2.35 \mathrm{O} 2+0.56 \mathrm{NH}_{3} \rightarrow 9.6$ P. putida $+2.4 \mathrm{CO}_{2}+2.96 \mathrm{H} 2 \mathrm{O}$ & Sucrose & $46 \%$ \\
\hline Sucrose + $3.8731 \mathrm{O}_{2} \rightarrow 1.48$ Muconic + $3.13 \mathrm{CO}_{2}+6.57 \mathrm{H} 2 \mathrm{O}$ & Sucrose & $54 \%$ \\
\hline Acetate $+.39 \mathrm{O} 2+0.093 \mathrm{NH}_{3} \rightarrow 1.6$ P. putida $+0.4 \mathrm{CO}_{2}+0.66 \mathrm{H} 2 \mathrm{O}$ & Acetate & $100 \%$ \\
\hline Extractives $+0.68 \mathrm{O} 2+0.28 \mathrm{NH}_{3} \rightarrow 4.8 P$. putida $+1.2 \mathrm{CO}_{2}+1.98 \mathrm{H} 2 \mathrm{O}$ & Extractives ${ }^{\mathrm{a}, \mathrm{b}}$ & $46 \%$ \\
\hline Extractives $+1.44 \mathrm{O}_{2} \rightarrow 0.74$ Muconic $+1.57 \mathrm{CO}_{2}+3.78 \mathrm{H} 2 \mathrm{O}$ & Extractives $^{\mathrm{a}, \mathrm{b}}$ & $54 \%$ \\
\hline Lignin $+3 \mathrm{O}_{2} \rightarrow 1$ Muconic $+2 \mathrm{CO}_{2}+1 \mathrm{H}_{2} \mathrm{O}$ & Lignin monomer & $100 \%$ \\
\hline
\end{tabular}

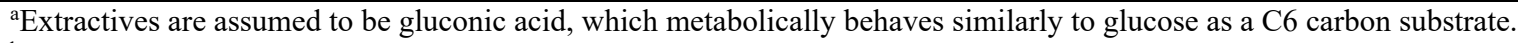

${ }^{\mathrm{b}} \mathrm{On}$ a mass basis biomass yield from extractives $=0.314 \mathrm{~g} / \mathrm{g}$ extractive, muconic yield $=0.289 \mathrm{~g} / \mathrm{g}$ extractive

The fermentation is run in a fed batch mode, with an assumed average working volume of $70 \%$. Broadly, the reactor is prefilled with diluted feed to $\sim 50 \%$, then the inoculum is transferred to the reactor. The reactor is then filled adjusting the rate of the concentrated feed to maintain a low but nonlimiting amount of carbon, which also improves the volume use over the course of the fermentation. When the maximum working volume of the column is reached, a portion of the broth is drawn, then fed batch operation proceeds. This cycle repeats for the duration of the fermentation, then the full volume of the reactor is drained.

The fermentation is controlled at a constant temperature of $32^{\circ} \mathrm{C}$ and operates at a mild positive pressure of $1.34 \mathrm{~atm}$, which assists in maintaining the axenic process. The current process assumes $1,000 \mathrm{~m}^{3}$ reactors with a length-to-diameter ratio of 6 translating to a diameter of $6 \mathrm{~m}$ and height of $35.8 \mathrm{~m}$. Overall, to completely convert the feed stream requires 17 bubble columns that are fed by 3 separate seed trains. Because the muconic acid product is an acid, it is necessary to consider the impact of $\mathrm{pH}$ during the fermentation. While evidence exists of $P$. putida tolerance in the basic environments more commonly found in soil environments (particularly if acclimated over a longer time), the inverse - tolerance to acidic conditions - is less well documented. To address this gap, the current design implements $\mathrm{pH}$ control to neutralize the acid produced during the fermentation (as needed in excess of the caustic in solution) administered in the pump-around loop after cooling the broth. The overall fermentation is targeted to occur at a net productivity of $1 \mathrm{~g}$ muconic $\mathrm{acid} / \mathrm{L} / \mathrm{hr}$ across all consumed substrates. The current model predicts net (i.e., including drawn volume/broth) cell titers at the end of the fermentation of $38 \mathrm{~g} / \mathrm{L}$, and muconic acid titers of $68.5 \mathrm{~g} / \mathrm{L}$. Table 26 lists the major reactor specifications for the seed and production system. 
Table 26. Key Operating/Design Parameters for Lignin Seed and Production Bioreactors

\begin{tabular}{lc}
\hline Parameter & Value \\
\hline Seed train & \\
Temperature, $^{\circ} \mathrm{C}$ & 32 \\
Pressure, atm & 1.34 \\
Stage 1 volume & $0.3 \mathrm{~m}^{3}$ (80 gallon) \\
Stage 2 volume & $3 \mathrm{~m}^{3}$ (800 gallon) \\
Stage 3 volume & $100 \mathrm{~m}^{3}$ \\
$\quad$ Diameter & $2.8 \mathrm{~m}$ \\
Height & $16.8 \mathrm{~m}$ \\
Number of seed trains & 3 \\
& \\
Production reactor & \\
Temperature, ${ }^{\circ} \mathrm{C}$ & 32 \\
Pressure, atm & 1.34 \\
Volume & $1,000 \mathrm{~m}^{3}$ \\
$\quad$ Diameter & $6 \mathrm{~m}$ \\
Height & $35.8 \mathrm{~m}$ \\
Net fermentation productivity, $\mathrm{g} / \mathrm{L} / \mathrm{hr}$ & 1 \\
Net cell titer, g/L & 38 \\
Net muconic acid titer, g/L & 68.5 \\
\hline
\end{tabular}

After the fermentation, the collected broth is sent through an ultrafilter to remove debris and cell mass. The remaining solids are sent to wastewater treatment and eventually burned in the high-solids boiler. The recovered liquid is carbon filtered to remove coloring compounds then proceeds to the muconic acid recovery system. The muconic acid is acidified and recovered via low temperature crystallization in the acid form [122]. Existing and predicted solubility curves for muconic acid and adipic acid shown in Figure 15 illustrate the strong temperature dependency of solubility for the fully protonated species $[130,131]$. At temperatures above the freezing point of water, fully protonated muconic acid (and adipic) will crystallize forming a high purity solid phase that can be easily removed via centrifugation from the residual liquid broth. However, compared to adipic acid, the muconic acid is on the order of $\sim 10$ times less soluble at equivalent temperatures and crystallizes to near completion with minimal loss increasing carbon efficiency. 

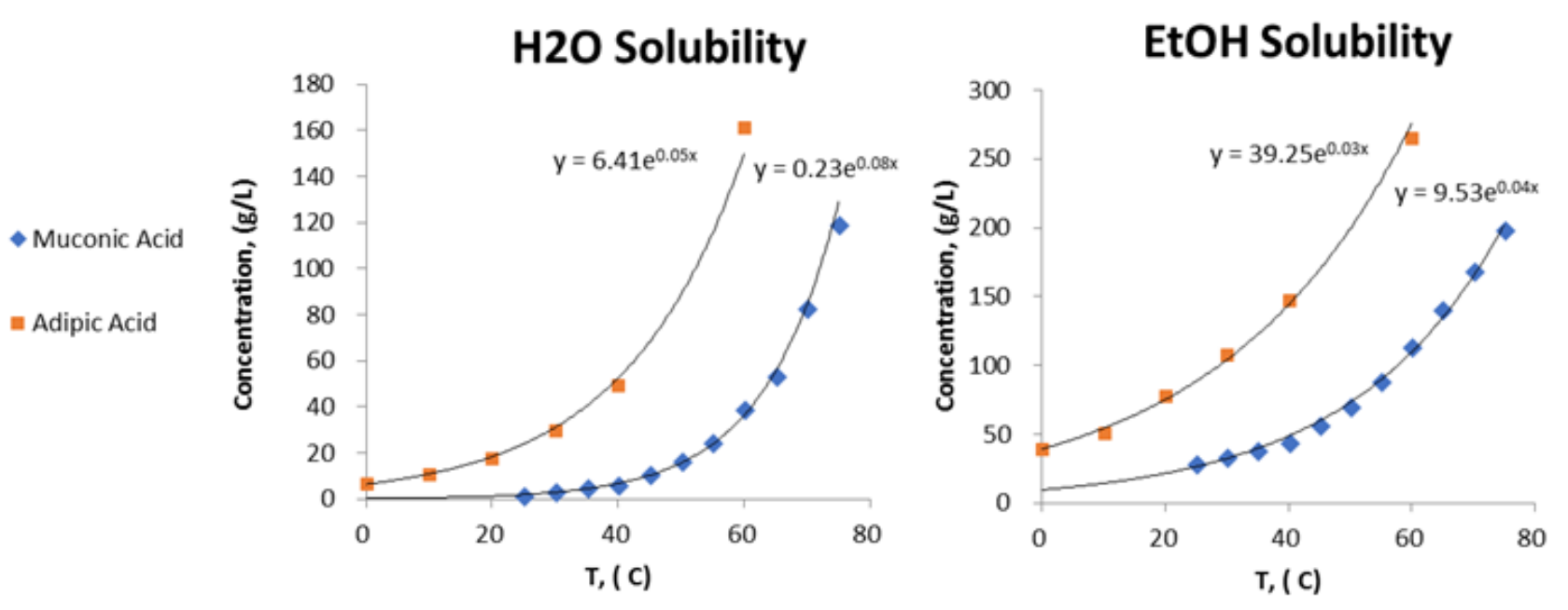

Figure 15. Predicted solubility curves for muconic and adipic acid

The current design assumes reacidification using sulfuric acid to drop the $\mathrm{pH}$ to 2 , where the muconic acid is in the protonated form. Rigorous $\mathrm{pH}$ calculations were performed at the predicted crystallization feed conditions and muconic acid $\mathrm{pKa}$ to understand the significance of $\mathrm{pH}$ variations, and it was determined that a $\mathrm{pH}$ of 2 should sufficiently ensure greater than $98 \%$ of the dissolved muconic acid will be in the protonated form. Because the second $\mathrm{pKa}$ of muconic acid is not well measured or reported, it was estimated using the second $\mathrm{pKa}$ of adipic acid ratioed by quotient of the first pKas. While this is not an accurate approach, at the low $\mathrm{pH}$ in the current design errors of $\pm 20 \%$ have minimal effect on the overall recovery. Figure 16 shows the species equilibrium as a function of $\mathrm{pH}$.

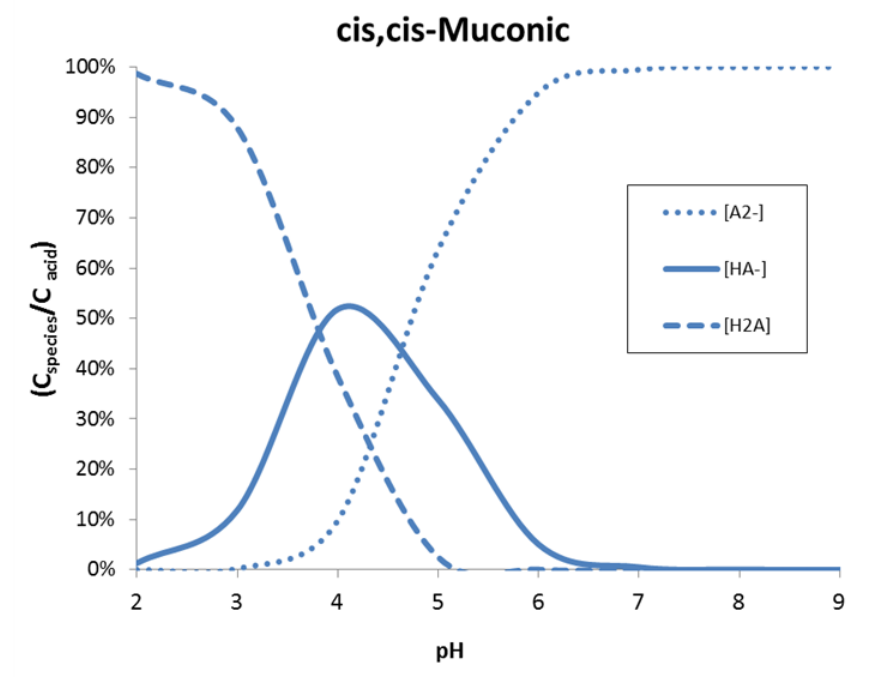

Figure 16. Acid-base equilibrium species at a given $\mathrm{pH}$

The crystallization occurs at a temperature of $15^{\circ} \mathrm{C}$, and a $\mathrm{pH}$ of 2 , recovering $98.8 \%$ of the product. The recovered crystal contains minor amounts of salt and water entrained in the crystal. The crystallizer mother liquid/broth is re-neutralized then sent to wastewater treatment where the salt is eventually separated and disposed of. The entrained liquid in the crystal is removed via a fluidized-bed 
drier, and then re-dissolved into an ethanol solvent. The ethanol:muconic acid ratio is set at 4 constrained to remain above the solubility limit of muconic acid in ethanol (Figure 14) at the mixing vessel temperature. Upon dissolution, the entrained salt ions precipitate out of the ethanol and are filtered prior to muconic acid upgrading. Table 27 lists the key crystallizer metrics.

Table 27. Muconic Acid Crystallizer Metrics

\begin{tabular}{lc}
\hline Parameter & Value \\
\hline Acid-base chemistry & \\
pKa 1 muconic acid & 3.87 \\
pKa 2 muconic acid (estimated) & $4.726\left(\sim 3.87 / 4.43^{*} 5.41\right)$ \\
pKa 1 adipic acid & 4.43 \\
pKa 2 adipic acid & 5.41 \\
$\mathrm{pKa}_{1} \mathrm{H}_{2} \mathrm{SO}_{4}$ & -3 \\
pKa2 $\mathrm{H}_{2} \mathrm{SO}_{4}$ & 1.99 \\
Muconic acid crystallizer operation & \\
Target $\mathrm{pH}$ & 2 \\
Temperature, ${ }^{\circ} \mathrm{C}$ & 15 \\
Pressure, atm & 1 \\
Percent recovery & $98.8 \%$ \\
ppm salt contamination & 13,400 \\
Configuration & Oslo continuous crystallizer \\
\hline
\end{tabular}

Adipic acid upgrading occurs in the liquid phase in a three-stage packed bed reactor operating at 40 atm. Hydrogen is fed in excess at a molar ratio of 2.6 mols $\mathrm{H}_{2}:$ mol muconic acid to ensure complete hydrogenation. The reactor is operated at a mild temperature of $78^{\circ} \mathrm{C}$ to avoid cracking of the facile double bond backbone, as well as over hydrogenation of the acid end groups critical to the final polymer properties. Inter-stage cooling is used to minimize temperature rise across each bed associated with the exothermic hydrogenation reactions. Complete hydrogenation is modeled based on experimental results over a $2 \%$ ruthenium on carbon catalyst $[122,123]$. After hydrogenation, the vapors are flashed and sent to the combustor, with the liquid proceeding to a flash evaporator which concentrates the adipic acid to a ratio of 2.5 ethanol:adipic by mass at elevated temperatures. The concentrated adipic acid product stream is crystallized by lowering the temperature to $15^{\circ} \mathrm{C}[132$, 133]. $73.4 \%$ of the adipic acid is removed per pass as crystals via centrifugation, and the mother liquor with the remaining uncrystallized adipic acid and ethanol is recycled back to the evaporation cycle until extinction. The recovered crystal product is sent to a drier to remove entrained ethanol, then stored on-site. Table 28 lists the key design parameters for the hydrogenation reactor and adipic acid crystallizer. 
Table 28. Muconic Acid Upgrading to Adipic Acid and Adipic Acid Recovery

\begin{tabular}{lc}
\hline Parameter & Value \\
\hline Hydrogenation reaction & \\
Ethanol:Muconic acid mass ratio & 4 \\
Temperature, ${ }^{\circ} \mathrm{C}$ & 78 \\
Pressure, atm & 40 \\
$\mathrm{H}_{2}:$ Muconic molar ratio & 2.6 \\
Muconic acid conversion & $100 \%$ \\
Catalyst & $2 \% \mathrm{Rh} / \mathrm{C}$ \\
WHSV (feed mass flow/catalyst mass) & $5 \mathrm{hr}^{-1}$ \\
& \\
Adipic acid crystallizer operation & \\
Configuration & Oslo continuous crystallizer \\
Ethanol:Adipic ratio feed & 5.3 \\
Ethanol:Adipic ratio concentrated & 2.5 \\
Crystallization temperature, ${ }^{\circ} \mathrm{C}$ & 15 \\
Pressure, atm & 1 \\
Single-pass adipic acid recovery & $73.40 \%$ \\
\hline
\end{tabular}

\subsubsection{Cost Estimation}

The BCD process occurs in a $127 \mathrm{~m}^{3}$ pulping reactor vertical pressure vessel with a design similar to the pre-steaming section of the dilute acid pretreatment reactor [10]. The vessel metallurgy was designed using 316 stainless steel as the material of construction with the appropriate vessel wall thickness for the elevated pressure.

All fermentation units are constructed of 304 or 316 stainless steel. Stainless steel is necessary to achieve the corrosion resistance to caustic solutions, as well as axenic fermentations where cleaning, contamination, and improved weldability are important considerations. The initial two seed fermentors maintained the same cost basis as the 2011 design report, quoted by A\&B Process Systems Corp. as skidded units with cooling jackets and agitators included. The third seed unit and production unit are bubble column reactor units with a length-to-diameter ratio of $6\left(100 \mathrm{~m}^{3}\right.$ unit: $35.8 \mathrm{~m}$ height, $6 \mathrm{~m}$ diameter) estimated through a combination of industry quotations and ACCE cost modeling. The bubble column reactors are sized to include a headspace allowance for expansion and vapor disengagement and use a pump-around loop to maintain the proper fermentation conditions. Fermentation compressors were sized based on the required air flow rate for meeting the OUR demands, with a pressure increase determined by the reactor dimensions and hydraulic pressure at the bottom of the broth ( $\sim 45 \mathrm{psig})$. Air compressors were constructed of carbon steel owing to the lack of corrosive chemical components.

Ultrafiltration and carbon filtration units were estimated based on guidance from an engineering consultancy with Nexant and designed as a counter current diafiltration package unit including feed pumps and controls. The unit is sized for a base membrane area of $53,820 \mathrm{ft}^{2}$ and includes an operating cost for membrane replacement. The carbon filtration unit has two beds and is constructed of carbon steel with stainless steel cladding to resist the corrosive acidic solution. The process stream flows through the first, while the second is regenerated using high pressure steam. The cycle time for each bed is assumed to be 1 hour. Granular active carbon was costed separately and replaced assuming a 3year lifetime. 
The crystallizer, centrifuge, and crystal drier unit capital costs were scaled from estimates provided by Nexant. The muconic acid crystallizer is designed as a continuous two-stage process occurring in Oslo type units that implement circulation cooling loops to maintain the constant temperature control required for the process. To recover high amounts of muconic acid a chilled-water heat exchanger was used, and the heat of reaction due to $\mathrm{pH}$ adjustment is accounted for. The muconic crystal recovery centrifuge operates continuously with a wash. Crystal dryers are designed as fluidized-bed units that circulate filtered hot air over the crystals (based on the information provided by Nexant). The design includes two parallel units sharing a single hot air unit.

The adipic acid hydrogenation reactor was quoted as a low-pressure fixed bed hydrodeoxygenation unit. The base reactor price quote was estimated through a separate NREL study of existing hydrotreater facility capital costs and correlations to liquid feed volume flow [134-142]. The base conditions for the hydrotreater estimate, pressures $>1,000 \mathrm{psig}$ and temperatures $>370^{\circ} \mathrm{C}$, were deescalated using the Guthrie pressure factor approach and the reactor size was scaled according to the total liquid volume (ethanol and muconic acid) entering the reactor[139]. Inter stage coolers, economizer heat exchangers, combined heat and power (CHP) systems, flash separators, and pumps were costed using ACCE[103]. In addition, the five-stage reciprocating makeup hydrogen compressor with a backup spare unit was costed using ACCE.

The adipic acid concentrator was designed as an initial mixing/feed tank, followed by a heat exchanger and flash evaporation tank constructed of SS316 for additional corrosion resistance. Adipic acid crystallizers, centrifuges, and dryers assumed similar designs as the muconic acid unit but scaled to the proper product stream.

\subsubsection{Achieving the Design Case}

Lignin conversion to value-added chemicals and materials has substantial promise to improve the overall economics of a lignocellulosic biorefinery, beyond its value as a boiler fuel. Moreover, the valorization of lignin is a critical need for ultimately enabling the lignocellulosic bioeconomy given that it can comprise up to a third of the carbon in plant biomass. This problem is incredibly challenging and has been actively researched for at least a century, with only a few options to date being economically viable, primarily including vanillin and lignosulfonates.

The approach described here, namely the use of a lignin depolymerization technology - a microbe to funnel a heterogeneous mixture of lignin-derived compounds to a single intermediate - and catalytic upgrading to a final product is a relatively new and potentially promising approach to valorize lignin $[107,114,143]$, but multiple technical challenges remain. The process modeled here uses BCD as a baseline approach for lignin depolymerization; this method has long been studied, but at low severity the monomer yields remain too low and at high severity the stream becomes quite toxic for microbial conversion. Broadly, one of the key limitations to date with a general approach of an intermediate biological step is the lack of robust catalytic technologies to depolymerize multiple bond types in lignin and provide sufficient bioavailable lignin-derived monomers. Specifically, aryl-ether bond cleavage is relatively well-known chemistry - for example, via reductive or oxidative routes [106, 144] - but lignin from most bioenergy feedstocks also exhibits a high fraction of C-C bonds.

Developing robust catalytic technologies to cleave both C-O and C-C bonds in lignin will be critical to ensuring a high yield of bioavailable compounds, one of the key limitations in reaching high yields of final products. 
In terms of the metabolic engineering aspects, substantial work remains in the development of robust microbes that are tailored to the lignin stream of interest for valorization. Significant efforts are now going into this problem worldwide, primarily via the use of non-model strains that harbor native aromatic catabolic capacity, such as $P$. putida, Rhodococci, and others. The process-relevant parameters of titer, rate, yield, and product recovery are of course key cost drivers. Similar to sugar conversion using pretreated hydrolysates, but likely more acute, aromatic compounds are typically quite toxic to microbes, and thus another important research area in this vein is in improving robustness of the chassis of choice for biological funneling. Beyond this, these types of streams may contain not only lignin, but also high content of extractives, sugar degradation products, or even sugars. Expanding the capacity of a microbe to truly convert all of these products into a single intermediate in an atom-efficient way remains a considerable challenge. While the current design implements a monoculture, the opportunities here for co-cultures or mixed cultures are considerable as well. Additionally, in the presence of solubilized lignin oligomers, some aromatic-catabolic bacteria have been shown to be able to depolymerize oligomers [143], but the mechanisms for this also remain unknown. The interface between the upstream catalysis and the microbial conversion step, overall, remain an area of active and intense investigation and likely will be the critical driver for the long-term viability, or lack thereof, of a biological lignin valorization approach.

Beyond the use of a microbial conversion step, the concept of a "lignin-first" biorefinery has emerged in the last decade $[106,144]$. One variation of this approach pursued at NREL, dubbed "reductive catalytic fractionation," or RCF, uses a polar protic solvent, such as methanol, at high temperatures to selectively solubilize lignin from whole biomass and passes it over a reducing catalyst in the presence of hydrogen or a hydrogen donor (such as a secondary alcohol or hemicellulose-derived sugars). RCF has created excitement in the lignin valorization community because it is an active stabilization method that can be tuned to yield complete aryl-ether bond cleavage and a narrow slate of aromatic monomers and easily digestible, delignified polysaccharides. RCF chemistry also holds promise for non-thermochemically pretreated biomass in which the polysaccharides have been removed, but where aryl-ether linkages are still intact. Multiple challenges remain for this approach to become viable and TEA will be critical to determine the primary cost drivers for these and any alternative approaches for lignin valorization.

Finally, the last decade has seen considerable improvements in the genetic engineering of bioenergy crops $[144,145]$. The overall outcome of these studies has been to demonstrate that lignin chemistry in planta is plastic and can be tuned in a way that maintains the efficacy of the overall plant. Even though ideally lignin valorization solutions will be developed that are feedstock agnostic, the variation in lignin chemistry between softwoods, hardwoods, and grasses is considerable, and even lignin chemistry variation within undomesticated species can be quite broad. Ideally, and in the long term, dedicated bioenergy crops will be selected and grown that not only maximize biomass and sugar yields, but also that have ideal lignin for valorization purposes while still achieving net improvements in sustainability. 


\subsection{Area 800: Combustor, Boiler, and Turbogenerator}

\subsubsection{Overview}

The purpose of the combined heat and power (CHP) subsystem is to burn various organic byproduct streams to produce steam and electricity. Combustible byproducts include unconverted lignin and carbohydrates from the feedstock, cell mass from fermentation and WWT, and off-gas streams from catalytic upgrading operations. Combustion of these byproduct streams generates steam to drive the upgrading and separation operations, and partially offsets the plant's electric power demand. It also reduces solid waste disposal costs and satisfies any regulatory issues with the handling of genetically engineered organisms.

Compared to previous biochemical design reports, the case for an integrated CHP system in the present design is less strong. In earlier designs, combustion of the unconverted lignin and extractives fractions provided (in many cases) sufficient heat and power to drive the entire process, with some excess power that could be exported as a coproduct. With the inclusion of lignin fermentation and upgrading technology in the present design, combined with the associated loss of extractives in the lignin fermentation process, the amount of residual solid fuel left for combustion is significantly reduced, and anaerobic digestion biogas is eliminated (because the anaerobic digestion unit is removed). Still, because there are residual waste solids still remaining after the lignin upgrading process as well as wastewater sludge (which would create a disposal problem if they could not be combusted on-site, including the use of engineered organisms), and because of the continued need for utility steam heating, the CHP area was retained in the present design and includes the same unit operations as before. Moreover, there is considerably higher use of sodium throughout the facility (i.e. DMR pretreatment, $\mathrm{BCD}$ lignin deconstruction, muconic acid fermentation, and ion exchange regeneration), the majority of which goes to WWT as soluble salts, but it is likely that sodium levels reaching the boiler with cake solids will also be higher than prior designs, increasing the risk for boiler slagging. Thus, if the solids boiler continues to be maintained in future designs, these issues must be further investigated with inputs from engineering subcontractors or boiler vendors. Accordingly, Area 800 remains an area for future optimization and cost reduction.

The fuel streams are fed to a combustor capable of handling the wet solids. A fan moves air into the combustion chamber. Treated water enters the heat exchanger circuit in the combustor and is boiled and superheated to high-pressure steam. A multistage turbine and generator are used to generate electricity. Steam is extracted from the turbine at two different conditions for use in the process. In the final stage of the turbine, the remaining steam is taken down to a vacuum and condensed with cooling water for maximum energy conversion. The condensate is returned to the boiler feed water system along with condensate from the various process heat exchangers. The steam turbine turns a generator that produces $\mathrm{AC}$ electricity for all users in the plant. NOx emissions are mitigated with ammonia injection in a selective non-catalytic reduction (SNCR) system, and $\mathrm{SO}_{\mathrm{x}}$ emissions are mitigated with flue-gas desulfurization.

\subsubsection{Design Basis}

The 2011 design report presented a CHP system comprising a high-solids, bubbling fluidized-bed boiler raising high-pressure (HP) steam at $900 \mathrm{psig}$, and a condensing steam turbine with two steam extractions at 175 psig and 125 psig. The boiler was based on the Babcock \& Wilcox Towerpak product, which is only able to raise steam at a single pressure. In both the acids and the BDO cases, the upgrading and distillation operations after fermentation required significantly higher temperatures than 
were specified in the previous design reports. In the acids case, some of the process temperatures were impossible to reach with steam at any pressure, so fired heaters were specified, with HP steam preheat and trim. In the BDO case, however, the oligomerization reactor requires a significant amount of heat at $250^{\circ} \mathrm{C}$. This is well served by 900 psig steam and consumes about $40 \%$ of the total steam thus produced. At this pressure, piping becomes more expensive and it is optimal to locate the process nearby the boiler to minimize piping runs; this is a consideration beyond the engineering feasibility level of analysis in the present design, but is worth noting for future work. An alternative could be a similar hot oil system or natural gas-fired heaters, which may be a more practical solution but would require consideration of LCA tradeoffs given high heat demands required for these heaters.

Apart from the limited number of HP steam users, the next level of steam required in either process is $175 \mathrm{psig}$, which is used for heating biomass feed to pretreatment temperatures by direct injection. Because the boiler is limited to a single steam pressure, and because both cases require some 900 psig steam, the steam turbine and electrical generator from earlier designs were retained to capture some energy from this pressure reduction. In future designs of the acid case, the relatively small trim duties could be absorbed in the specified fired heaters and the production of HP steam could be eliminated, reducing the boiler and feed water treatment capital costs, with the tradeoff of slightly higher natural gas costs. The BDO case is not so straightforward, given the high HP steam duty requirement of the oligomerization reactor. A fired heater or secondary HP boiler operating on natural gas could be used here as well, but the capital and operating cost tradeoffs are more difficult to determine, given the efficiencies of each. Ultimately, this is a minor sensitivity compared to other uncertainties in either process so, for simplicity and comparison, the CHP system was left the same as in previous design reports.

The details of the CHP system design can be found in the 2011 report. To briefly summarize, the design uses a bubbling fluidized-bed combustor capable of complete combustion of the wet solid fuel and gas streams, along with any supplemental natural gas. Flue gas from the combustor preheats the entering combustion air then enters a spray dryer for flue gas desulfurization (FGD). Ash is removed in a baghouse and disposed to a landfill. Finally, the boiler raises steam at $900 \mathrm{psig}$, which is superheated and then sent through a multistage steam turbine with two extraction ports and a final condenser. The steam turbine turns an electric generator to offset some of the power demand in the process. The balance of electric power is assumed to be purchased from the grid (see Area 900).

\subsubsection{Cost Estimation}

For the 2011 design report, Harris Group worked with Babcock \& Wilcox and Siemens to provide capital cost quotes for the biomass boiler and condensing extraction turbine and generator. The cost basis for the A800 equipment remains the same as described in the 2011 ethanol report. The boiler capital cost includes the boiler feed water preheater, FGD spray dryer, and baghouse. For the baghouse, bag replacement appears as a periodic charge in the cash flow worksheet. Harris Group also obtained quotes from a third vendor for support equipment including the deaerator, chemical injection system, tanks, and pumps. 


\subsection{Area 900: Utilities}

\subsubsection{Overview}

Area 900 tracks all plant utilities except steam, which is provided by Area 800, including electric power, cooling water, chilled water, plant and instrument air, process water, and the CIP system. The process water manifold in Area 900 mixes fresh water with treated wastewater and condensate from the sugar evaporation system (assumed suitable for all plant users) and provides this water at a constant pressure to the facility. Water is provided to the cellulase production unit, boiler and cooling tower makeup, the CIP system, and the wash for the lignin filter press. Fresh water is also mixed with some internally recycled water for dilution before pretreatment and enzymatic hydrolysis. The plant and instrument air systems provide compressed air for general use (pneumatic tools and clean-up) and instrument operation. Larger users of compressed air, namely the cellulase system and aerobic bioreactors, have their own compressors specified. The CIP system provides hot cleaning and sterilization chemicals to hydrolysis, bioconversion, and the enzyme production section.

\subsubsection{Design Basis}

The cooling water system is designed for a $28^{\circ} \mathrm{C}$ supply temperature with a $9^{\circ} \mathrm{C}$ temperature rise in coolers throughout the facility. This is an assumed average rise; the actual cooling water rises across each exchanger are not explicitly modeled in Aspen. The primary cooling water users option are described in Table 29, and their contributions are compared in Figure 17.

Table 29. Major Cooling Water Users

\begin{tabular}{ll}
\hline Evaporator condensate cooler & Provides cooling of the sugar evaporator condensate (acids case only). \\
\hline Lignin upgrading coolers & Trim cooling duty used in the lignin conversion/upgrading area. \\
\hline Turbine condenser & Condenses the steam turbine exhaust at a vacuum. \\
\hline Chiller condenser & $\begin{array}{l}\text { The chilled-water loop requires cooling water to condense the refrigerant. } \\
\text { The cooling water duty to M-908 is set equal to the total load on the chilled- } \\
\text { water loop. In Figure 17, the chiller contributions are divided by area. }\end{array}$ \\
\hline
\end{tabular}

As noted in Section 3.8, the heat and power balance has changed significantly since the 2013 design report, so the condensing turbine is no longer the largest user of cooling water. In fact, the steam system becomes heat limited for the more heat-intensive BDO pathway (associated with energy demands around aqueous BDO upgrading) and must import natural gas to satisfy facility heat requirements, leaving minimal steam routed through the final condensing turbine stage. The single largest user is the chiller condenser, which services the chilled-water loop used in Areas 300, 400, and 700, driven primarily by Area 700 cooling demands; at the cooling temperatures dictated in Area 700, it would be difficult to avoid chilled water utilities for this section. The compressor electricity demand for the chiller was estimated at $0.56 \mathrm{~kW} /$ ton of refrigeration and the cooling water demand for the chiller system was assumed to be equal to the heat removed in the chilled-water loop.

The cooling tower evaporation rate is based on a temperature drop from $37^{\circ} \mathrm{C}$ to $28^{\circ} \mathrm{C}$. Windage was taken to be $0.005 \%$ of the total flow to the tower and cooling tower blowdown was assumed to be $0.15 \%$ of the flow leaving the tower basin. 

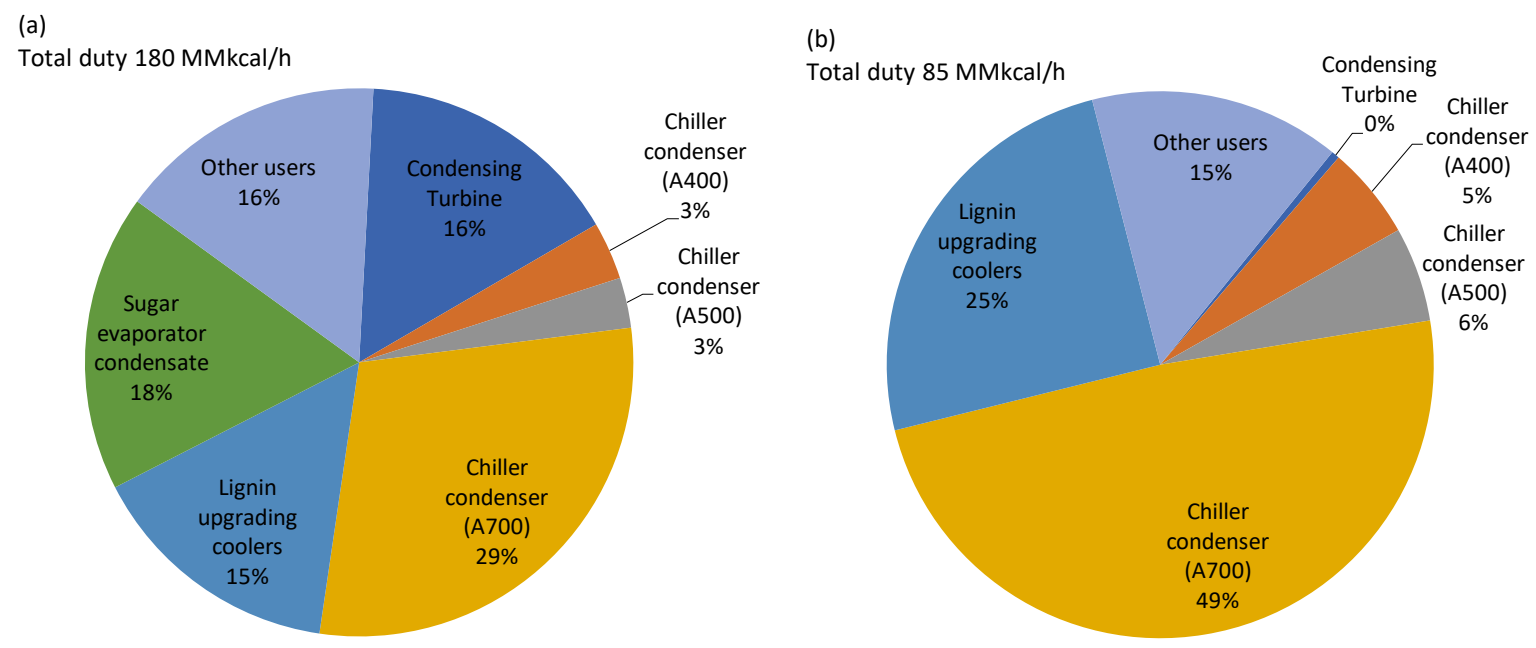

Figure 17. Cooling water heat duty distribution between major users; (a) acids case, (b) BDO case

Fresh water is assumed to enter the facility at $13^{\circ} \mathrm{C}$ and provides supplemental cooling to the plant before entering the process water tank. The fresh water is mixed with the treated wastewater effluent and the evaporation condensate in the process water tank (T-914) and then split several ways. Clean water must be provided to biomass dilution in the front end, to the cellulase production unit, to the washing filters, and to the boiler and cooling tower water makeup. The process water tank is designed for an 8-hour residence time. The process water pump (P-914) pumps water from the tank into the facility and is designed to handle 1.5 times the process water flow requirement.

The plant and instrument air systems provide compressed air for pneumatic tools and clean-up and instrument operation (not including major air demands such as enzyme and product bioreactors). The plant air compressor is sized for 400 SCFM at 125 psig. An instrument air dryer and surge tank were designed to provide clean dry air at a consistent pressure to the instrument air system. The surge tank was sized at 3,800 gallons.

The electricity generated in Area 800 is used to partially offset the plant power demand throughout the facility to power pumps, agitators, compressors, etc. (65.2 MW total power required for the acids case and 54.2 MW for the BDO case), but there is still a considerable power deficit on the order of roughly 42-44 MW that must be imported from the grid after considering the power generated on-site. The distribution of total plant power utilization among all areas is shown in Figure 18. Note that the cost of the power required by Area 100 is already assumed to be included in the feedstock cost and is subtracted from the plant's net electricity import. This is reflected in the economics by an operating cost credit equal to this amount of electricity.

In both pathways, Area 200 constitutes a sizeable power demand, primarily due to the switch to DMR pretreatment in this design, which trades heat demands that were required more substantially for DDA pretreatment with power demands to drive the mechanical refining equipment. Additionally, Area 300 constitutes a large power consumer for the acids pathway, primarily due to the addition of continuous enzymatic hydrolysis and subsequent MVR evaporation units (dictated most strongly by the latter, 
with MVR evaporators driven by power rather than heat). In the future, steam rather than MVR evaporators may be further considered for cost versus energy usage tradeoffs.

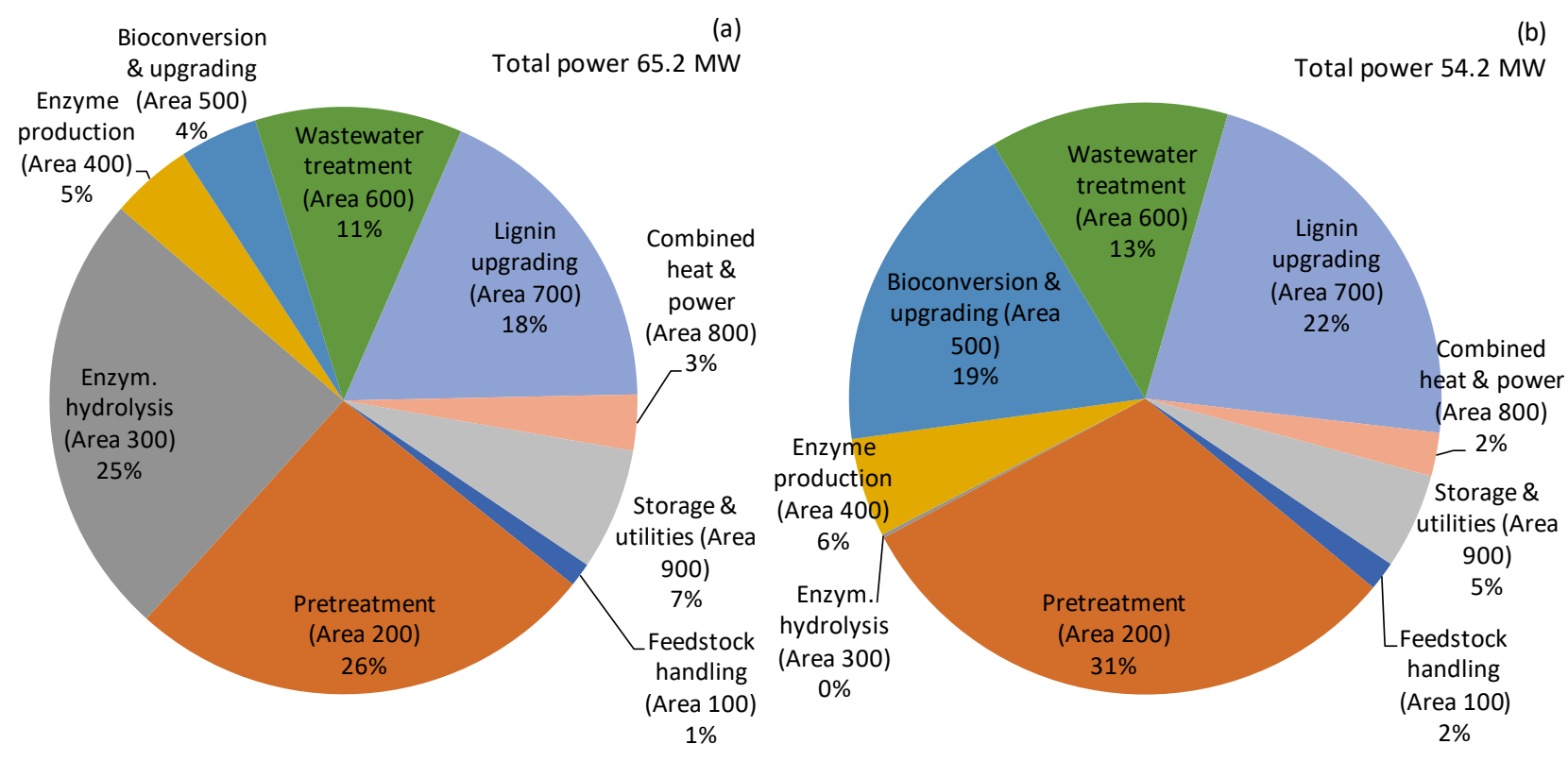

Figure 18. Distribution of plant electricity utilization by process area; (a) acids case, (b) BDO case

\subsubsection{Cost Estimation}

All cost estimates for the utility equipment in A900 were maintained consistent with the basis values used in the 2011 report. To summarize, the cooling tower was based on a cost estimate from a vendor for a fiberglass cooling tower capable of handling 44,000 gal $/ \mathrm{min}$; this cost is scaled to the respective cooling water throughputs estimated here. Harris Group had estimated costs for the cooling water circulation and makeup pumps using their internal database. The material of construction for the cooling water loop is carbon steel. The cost for the chiller came from a recent quote for a similarly sized system. Harris Group had also used their internal database to estimate costs for the remaining equipment: the process water tank and pump; the plant/instrument air compressor, dryer, and surge tank; and the CIP system. 


\section{Process Economics}

The ultimate purpose for developing such a detailed process design, simulation model, and cost estimate is to determine the economics of biofuel production in an integrated biorefinery. This information is used either as an absolute cost to assess the product's potential in the marketplace or as a relative cost that can be used to guide research by examining the change in production cost associated with a process modification or other core research activity.

The total capital investment (TCI) is first computed from the total equipment cost (estimated per above based on process flows which allow for designing/sizing all equipment and associated capital costs). Next, variable and fixed operating costs are determined. With these costs, we use a discounted cash flow analysis to determine the minimum fuel selling price required to obtain a zero net present value (NPV) with a fixed internal rate of return (IRR). This section summarizes the assumptions made in completing the discounted cash flow analysis, with more details and supporting description available in the 2011 ethanol design report [2] and 2013 biological hydrocarbon design report [10] for assumptions that are unchanged. Our analysis does not take into account any policy factors such as subsidies, mandates, or carbon credits, because these would be speculative. The purpose of this analysis is to demonstrate the process requirements needed to achieve specific DOE cost targets which are set from the top down, and to demonstrate how the technology pathway described here is able to achieve such targets on its own merits (through bottom-up TEA modeling) and, if it cannot, to give stakeholders a sense of the magnitude of incentive required to make it so.

\subsection{About Cost-Year Indices}

The cost-year of 2016 was chosen for this analysis to provide more updated and relevant cost output information relative to prior efforts in $2011 \$$ and $2014 \$[10,146]$. This new basis is being applied consistently across all DOE-BETO platforms for which similar design case target projections are being established to support upcoming Multi-Year Program Plan (MYPP) efforts, to permit comparison of future feedstocks, conversion technologies, and other alternative scenarios. However, equipment and material costs are largely based on information obtained in previous years, which must then be indexed to 2016. As presented in prior design cases and MYPP reports [2, 146], capital costs were adjusted using the Plant Cost Index from Chemical Engineering Magazine [147] to a common basis year of 2016. Similarly, for chemical/material costs we used the Producer Price Index (PPI) for chemical manufacturing published by the U.S. Bureau of Labor Statistics [148]. Employee salaries were maintained from prior basis values and were scaled to 2016 using the labor indices provided by the U.S. Bureau of Labor Statistics [148]. The general formula for cost-year dollar back-casting is:

$$
2016 \text { Cost }=(\text { Base Cost })\left(\frac{2016 \text { Cost Index }}{\text { Base Year Index }}\right)
$$

\subsection{Total Capital Investment}

Section 3 of this report describes the details of the conceptual process designs and how the purchased cost of the equipment was determined. The next step is to determine the installed cost of that equipment. The installation cost can be determined by performing a detailed study of everything required to install the necessary equipment and make it operational (e.g., foundation, piping, and wiring). This type of detail is not warranted at this level of process development, and a factored approach in which multipliers are applied to the purchased equipment cost is considered satisfactory. 
The methodology and rationale for applying unit-level installation costs remain the same as described in the 2011 and 2013 reports, and again, further detail can be found there that will not be repeated here.

In summary, each type of equipment utilizes a different installation factor to scale the given direct equipment purchased cost to a final installed cost. A complete list of the equipment is provided in Appendix A, along with equipment purchased and installed costs. The purchased cost for a given component reflects a baseline equipment size. As changes are made to the process, the equipment size required may be different than what was originally designed and costed. Instead of re-costing in detail, an exponential scaling expression was used:

$$
\text { New Cost }=(\text { Base Cost })\left(\frac{\text { New Size }}{\text { Base Size }}\right)^{n}
$$

In this equation, the scaling exponent $n$ varies depending on the type of equipment to reflect economyof-scale dependencies (more detail on reasonable scaling values for different types of equipment is provided in the 2011 ethanol report [2]). The basis for scaling is typically some characteristic of the equipment related to production capacity, such as flow or heat duty. Some equipment does not follow, such a scaling-factor approach, namely when the capacity for a given operation is exceeded and requires multiple units in parallel, thus losing economy-of-scale benefits that are captured in the exponential expression above.

Once the total equipment cost has been determined in the year of interest, several other direct and indirect costs were added to determine the TCI. Site development and warehouse costs, along with additional piping, are based on the inside-battery-limits (ISBL) equipment costs (Areas 200, 300, 400, 500 , and 700 in this design) and are considered part of the total direct cost (TDC). Beyond the ISBL operations, the other process areas are considered outside battery limits (OSBL), including Areas 100 (rolled up into feedstock costs), 600, 800, and 900. Because TDC includes escalation factors only based on ISBL areas (see Table 30), the TDC is more inflated here relative to prior designs given the addition of the lignin-to-coproduct train (now Area 700), which is considered as additional ISBL operations, while Area 800 (lignin combustion/CHP) is considered as OSBL supporting operations.

Project contingency, field expenses, home office engineering and construction activities, and other costs related to construction are computed relative to the TDC and give the fixed capital investment (FCI) when summed. The sum of FCI and the working capital for the project is the TCI. Table 30 summarizes these categories and additional factors. The values assumed for each respective factor were maintained consistently with those discussed in the 2011 ethanol report [2] as well as the 2013 biological hydrocarbon report [10]. 
Table 30. Additional Costs for Determining TCI

\begin{tabular}{|c|c|c|}
\hline Item & Description & Amount \\
\hline \multicolumn{3}{|c|}{ Additional direct costs } \\
\hline Warehouse & On-site storage of equipment and supplies. & $4 \%$ of $I S B L^{a}$ \\
\hline $\begin{array}{l}\text { Site } \\
\text { development }\end{array}$ & $\begin{array}{l}\text { Includes fencing, curbing, parking lot, roads, well drainage, rail } \\
\text { system, soil borings, and general paving. This factor allows for } \\
\text { minimum site development assuming a clear site with no unusual } \\
\text { problems such as right-of-way, difficult land clearing, or unusual } \\
\text { environmental problems. }\end{array}$ & $9 \%$ of ISBL \\
\hline $\begin{array}{l}\text { Additional } \\
\text { piping }\end{array}$ & $\begin{array}{l}\text { To connect ISBL equipment to storage and utilities outside battery } \\
\text { limits. }\end{array}$ & $4.5 \%$ of ISBL \\
\hline \multicolumn{3}{|c|}{ Indirect costs } \\
\hline $\begin{array}{l}\text { Prorateable } \\
\text { costs }\end{array}$ & $\begin{array}{l}\text { This includes fringe benefits, burdens, and insurance of the } \\
\text { construction contractor. }\end{array}$ & $10 \%$ of TDC \\
\hline Field expenses & $\begin{array}{l}\text { Consumables, small tool and equipment rental, field services, } \\
\text { temporary construction facilities, and field construction } \\
\text { supervision. }\end{array}$ & $10 \%$ of TDC \\
\hline $\begin{array}{l}\text { Home office } \\
\text { and } \\
\text { construction }\end{array}$ & Engineering plus incidentals, purchasing, and construction. & $20 \%$ of TDC \\
\hline $\begin{array}{l}\text { Project } \\
\text { contingency }\end{array}$ & Extra cash on hand for unforeseen issues during construction. & $10 \%$ of TDC \\
\hline Other costs & $\begin{array}{l}\text { Includes: start-up and commissioning costs; land, rights-of-way, } \\
\text { permits, surveys, and fees; piling, soil compaction/dewatering, and } \\
\text { unusual foundations; sales, use, and other taxes; freight, } \\
\text { insurance in transit, and import duties on items such as } \\
\text { equipment, piping, steel, and instrumentation; overtime pay during } \\
\text { construction; field insurance; project team; and parameters such } \\
\text { as transportation equipment, bulk shipping containers, and plant } \\
\text { vehicles. }\end{array}$ & $10 \%$ of TDC \\
\hline
\end{tabular}

a ISBL = installed cost of equipment inside battery limits (A200, 300, 400, 500, and 700). 
Table 31. Project Cost Worksheet Including TDC and TCI (2016\$)

\begin{tabular}{|c|c|c|c|c|}
\hline \multirow[b]{2}{*}{ Process Area } & \multicolumn{2}{|c|}{ BDO Pathway } & \multicolumn{2}{|c|}{ Acids Pathway } \\
\hline & $\begin{array}{c}\text { Purchased } \\
\text { Cost }\end{array}$ & $\begin{array}{c}\text { Installed } \\
\text { Cost }\end{array}$ & $\begin{array}{c}\text { Purchased } \\
\text { Cost }\end{array}$ & $\begin{array}{l}\text { Installed } \\
\text { Cost }\end{array}$ \\
\hline Area 100: Feedstock Storage and Handling ${ }^{a}$ & \multicolumn{2}{|c|}{ Included in feedstock cost } & \multicolumn{2}{|c|}{ Included in feedstock cost } \\
\hline Area 200: Pretreatment & $\$ 35,800,000$ & $\$ 48,700,000$ & $\$ 35,800,000$ & $\$ 48,700,000$ \\
\hline Area 300: Enzymatic Hydrolysis and Hydrolysate Conditioning & $\$ 9,100,000$ & $\$ 15,400,000$ & $\$ 31,100,000$ & $\$ 62,700,000$ \\
\hline Area 400: Enzyme Production & $\$ 6,700,000$ & $\$ 11,500,000$ & $\$ 6,700,000$ & $\$ 11,500,000$ \\
\hline Area 500: Fermentation, Catalytic Conversion, and Upgrading & $\$ 34,800,000$ & $\$ 61,100,000$ & $\$ 24,300,000$ & $\$ 43,800,000$ \\
\hline Area 600: Wastewater Treatment ${ }^{b}$ & $\$ 19,000,000$ & $\$ 35,600,000$ & $\$ 21,000,000$ & $\$ 38,800,000$ \\
\hline Area 700: Lignin Upgrading & $\$ 60,800,000$ & $\$ 128,400,000$ & $\$ 60,300,000$ & $\$ 127,500,000$ \\
\hline Area 800: Combustor, Boiler, and Turbogenerator & $\$ 28,200,000$ & $\$ 51,000,000$ & $\$ 26,900,000$ & $\$ 48,700,000$ \\
\hline Area 900: Utilities & $\$ 9,000,000$ & $\$ 15,500,000$ & $\$ 9,900,000$ & $\$ 17,200,000$ \\
\hline Totals (Excl. Area 100) & $\$ 203,500,000$ & $\$ 367,200,000$ & $\$ 217,000,000$ & $\$ 398,900,000$ \\
\hline Warehouse & of ISBL & $\$ 10,600,000$ & & $\$ 11,800,000$ \\
\hline Site Development & of ISBL & $\$ 23,900,000$ & & $\$ 26,500,000$ \\
\hline Additional Piping & of ISBL & $\$ 11,900,000$ & & $\$ 13,200,000$ \\
\hline Total Direct Costs (TDC) & & $\$ 413,600,000$ & & $\$ 450,400,000$ \\
\hline Prorateable Expenses & of TDC & $\$ 41,400,000$ & & $\$ 45,000,000$ \\
\hline Field Expenses & of TDC & $\$ 41,400,000$ & & $\$ 45,000,000$ \\
\hline Home Office and Construction Fee & of TDC & $\$ 82,700,000$ & & $\$ 90,100,000$ \\
\hline Project Contingency & of TDC & $\$ 41,400,000$ & & $\$ 45,000,000$ \\
\hline Other Costs (Start-Up, Permits, etc.) & of TDC & $\$ 41,400,000$ & & $\$ 45,000,000$ \\
\hline Total Indirect Costs & & $\$ 248,200,000$ & & $\$ 270,200,000$ \\
\hline Fixed Capital Investment (FCI) & & $\$ 661,800,000$ & & $\$ 720,600,000$ \\
\hline Land & & $\$ 1,800,000$ & & $\$ 1,800,000$ \\
\hline Working Capital & of $\mathrm{FCl}$ & $\$ 33,100,000$ & & $\$ 36,000,000$ \\
\hline Total Capital Investment (TCI) & & $\$ 696,800,000$ & & $\$ 758,400,000$ \\
\hline Lang Factor (TCl/Purchased Equip Cost) & & 3.8 & & 3.9 \\
\hline $\mathrm{TCl}$ per Annual Gallon Gasoline Equivalent & & $\$ 22.27 / G G E$ & & $\$ 23.35 / G G E$ \\
\hline
\end{tabular}

${ }^{a}$ Feedstock handling not included in this calculation.

${ }^{\mathrm{b}}$ Area 600 not included in Lang factor.

\subsection{Variable Operating Costs}

Variable operating costs, which include raw materials, waste handling charges, and byproduct credits, are incurred only when the process is operating. Quantities of raw materials used and wastes produced were determined using the Aspen material balance. Table 32 documents the costs and sources of chemicals used in the process and Table 33 summarizes the variable costs on a per-year and per-GGE basis. The cost basis for most material costs in the present model that were also used in previous 2013 or 2011 design case models were left unchanged, aside from indexing to 2016\$. However, larger-cost items were revisited based on the most recent information available, including ammonia, sulfuric acid, glucose (concentrated glucose syrup), and diammonium phosphate, all updated based on averaging the most recent five-year cost history for each item (based on industry databases for all but glucose syrup, which was based on USDA sugar price data [149]). The cost of sodium hydroxide was also updated based on guidance from a recent subcontract with Nexant, set at $\$ 0.23 / \mathrm{lb}$ in $2011 \$$, who also provided costs for sodium sulfate byproduct recovery equipment (discussed in Section 3.6) and resultant sodium 
sulfate salt selling price, set at $\$ 0.07 / \mathrm{lb}$ in $2011 \$$. As noted previously, the decision was made in this design to sell sodium sulfate salt after further purification of the WWT brine stream, given the substantial use of caustic and acid throughout the facility (i.e. during DMR pretreatment, lignin BCD, and muconic acid fermentation and recovery), which incurs significant costs equating to over \$1.30/GGE MFSP penalties for either fuel pathway, and would add another \$0.30/GGE for disposal (landfilling) of the waste brine. As this is untenable for ultimately achieving \$2.50/GGE MFSP targets, a portion of those costs are offset by selling the sodium sulfate byproduct.

Both pathways require a net power import after considering the amount of power generated through the CHP system, costed consistently with prior design cases with grid imports at $6.8 ф / \mathrm{KWh}$ (still reflective of current retail prices for the industrial sector as published by the Energy Information Administration [150]). Given a considerably larger usage of acids and bases in the present design, the cost for solids disposal (boiler ash and salt from WWT) is increased, with both ash and salt disposed to landfilling. Where needed, hydrogen was assumed here to be purchased as a product from standard natural gas-derived steam methane reforming (SMR) consistent with the 2013 report which utilized hydrogen for hydrotreating fatty acids. Net makeup hydrogen demands were calculated after including hydrogen co-produced and purified from both pathways' anaerobic fermentation steps; in the acids case, this translated to more hydrogen produced than required with the excess sent to the boiler, while the $\mathrm{BDO}$ case required additional net hydrogen import. The purchased hydrogen price was set based on a recent DOE Hydrogen and Fuel Cells Program report, which presented a current price for natural gas-based SMR hydrogen of $\$ 1.57 / \mathrm{kg}$ (assumed in 2012\$) associated with a natural gas price of \$4/MM BTU [151], which was applied consistently following the 2013 biological hydrocarbon report. As this represents an SMR plant-gate price, this implies that the hydrogen plant is assumed to be colocated nearby with minimal costs for transportation "over the fence" to the biorefinery.

Adipic acid is co-produced in this design at a product purity over $99.7 \mathrm{wt} \%$, with a sale price set at $\$ 1,710 /$ short ton $(\$ 0.86 / \mathrm{lb}$ ) in $2016 \$$. This aligns with purity requirements of $99.8 \%$ for production of nylon-6,6 via adipic acid as outlined in Vardon et al. [122]. Estimating prices for commodity chemicals is challenging as these prices vary on a quarterly, if not daily, basis. The cause for price variability is product specific and the reasons for these fluctuations range from impacts of feedstock costs to shifts in supply/demand, to unplanned outages at production facilities, to name a few common examples. Given the large impact that this coproduct price has on the underlying MFSP, this analysis strives to avoid any bias in the underlying assumption on commodity price cycles by a) utilizing a price reflective of the average value over the largest amount of data we have available, with the $\$ 1,710 /$ ton value attributed to a 15 -year price average, and b) employing sensitivity analysis to evaluate the impact of the assumed price on the underlying MFSP. At the time of developing this write-up, the latest reported price of adipic acid in 2017 (US Gulf Coast) was roughly $\$ 1,300 /$ ton. Shifting the chemical price from $\$ 1,710$ to $\$ 1,300 /$ ton would increase the MFSP by over $\$ 1 /$ GGE. However, between the period of 2011 to 2015 , the price of adipic acid fluctuated between $\$ 2,300 /$ ton and $\$ 960 /$ ton, in part reflecting trends in petroleum prices. Given this typical correlation between commodity chemicals and petroleum prices, it bears noting that relative to that same 2017 reference point at $\$ 50 /$ barrel oil, crude prices are projected by the U.S. Energy Information Administration to rise by $80 \%$ by 2030 (the target year tied to the $n^{\text {th }}$-plant $\$ 2.50$ /GGE MFSP goal for the facility to begin construction), or more than double by 2050 (year 20 of the subsequent 30-year facility lifetime), thus tempering possible concerns about the $\$ 1,710 /$ ton adipic acid price basis being optimistic over the plant lifetime relative to current market prices today. 
Table 32. Chemical Costs and Sources

\begin{tabular}{|c|c|c|}
\hline Component & Cost $(2016 \$)$ & Source \\
\hline Biomass delivered to reactor throat & $\$ 0.0285 / \mathrm{lb}$ & INL inputs, $\$ 71.26 /$ dry ton @ 20\% moisture \\
\hline Sulfuric acid, $93 \%$ & $\$ 0.0430 / \mathrm{lb}$ & Industry database, 5-year average \\
\hline Ammonia & $\$ 0.1900 / \mathrm{lb}$ & Industry database, 5-year average \\
\hline Sodium hydroxide & $\$ 0.2384 / \mathrm{lb}$ & Nexant (indexed from $2011 \$$ basis) \\
\hline Ultrafilter replacement & $0.0297 \$ / \$$ cost & Nexant (annual cost per $\$$ membrane capex) \\
\hline Corn steep liquor & $\$ 0.0339 / \mathrm{lb}$ & Corn Products via Harris Group \\
\hline Diammonium phosphate & $\$ 0.1645 / \mathrm{lb}$ & Industry database, 5-year average \\
\hline Corn oil (antifoam) & $\$ 0.6439 / \mathrm{lb}$ & Industry database \\
\hline Glucose & $\$ 0.3670 / \mathrm{lb}$ & USDA ERS, 5-year average [149] \\
\hline $\mathrm{SO}_{2}$ & $\$ 0.1811 / \mathrm{lb}$ & Industry database \\
\hline Enzyme nutrients & $\$ 0.4896 / \mathrm{lb}$ & $\begin{array}{l}\text { Industry database (See } 2011 \text { design report for } \\
\text { details) }\end{array}$ \\
\hline Hydrogen & $\$ 0.7306 / \mathrm{lb}$ & DOE report, SMR H2 @ \$4/MM BTU NG [151] \\
\hline Hot oil system (utility heating) & \$5/MM BTU & Driven by NG prices \\
\hline Toluene solvent (acids case) & $\$ 0.3303 / \mathrm{lb}$ & Industry database \\
\hline Ketonization catalyst (acids case) & $\$ 2.95 / \mathrm{lb}$ & NREL internal database \\
\hline Condensation catalyst (acids case) & $\$ 9.07 / \mathrm{lb}$ & NREL internal database \\
\hline HDO catalyst (acids case) & $\$ 651 / \mathrm{lb}$ & NREL internal database \\
\hline BDO upgrading catalyst (BDO case) & $\$ 70 / \mathrm{lb}$ & NREL internal database \\
\hline Oligomerization catalyst (BDO case) & $\$ 70 / \mathrm{lb}$ & NREL internal database \\
\hline HDO catalyst (BDO case) & $\$ 528 / \mathrm{lb}$ & NREL internal database \\
\hline Polymer for WWT & $\$ 2.6282 / \mathrm{lb}$ & Brown and Caldwell 2012 WWT design [152] \\
\hline Ethanol & $\$ 0.3370 / \mathrm{lb}$ & Prior NREL analysis \\
\hline Natural gas & \$5/MM BTU & Basis approximating historical NG prices \\
\hline Lime & $\$ 0.1189 / \mathrm{lb}$ & Harris Group \\
\hline Boiler chemicals & $\$ 2.9772 / \mathrm{lb}$ & 2002 Design Report [3] \\
\hline Cooling tower chemicals & $\$ 1.7842 / \mathrm{lb}$ & 2002 Design Report [3] \\
\hline Fresh water & $\$ 0.0002 / \mathrm{lb}$ & Peters and Timmerhaus [153] \\
\hline Sodium sulfate salt coproduct value & $\$ 0.0706 / \mathrm{lb}$ & Nexant (indexed from $2011 \$$ basis) \\
\hline Adipic acid coproduct value & $\$ 0.8554 / \mathrm{lb}$ & Average price over a 15 -year cycle \\
\hline
\end{tabular}


Table 33. Variable Operating Costs

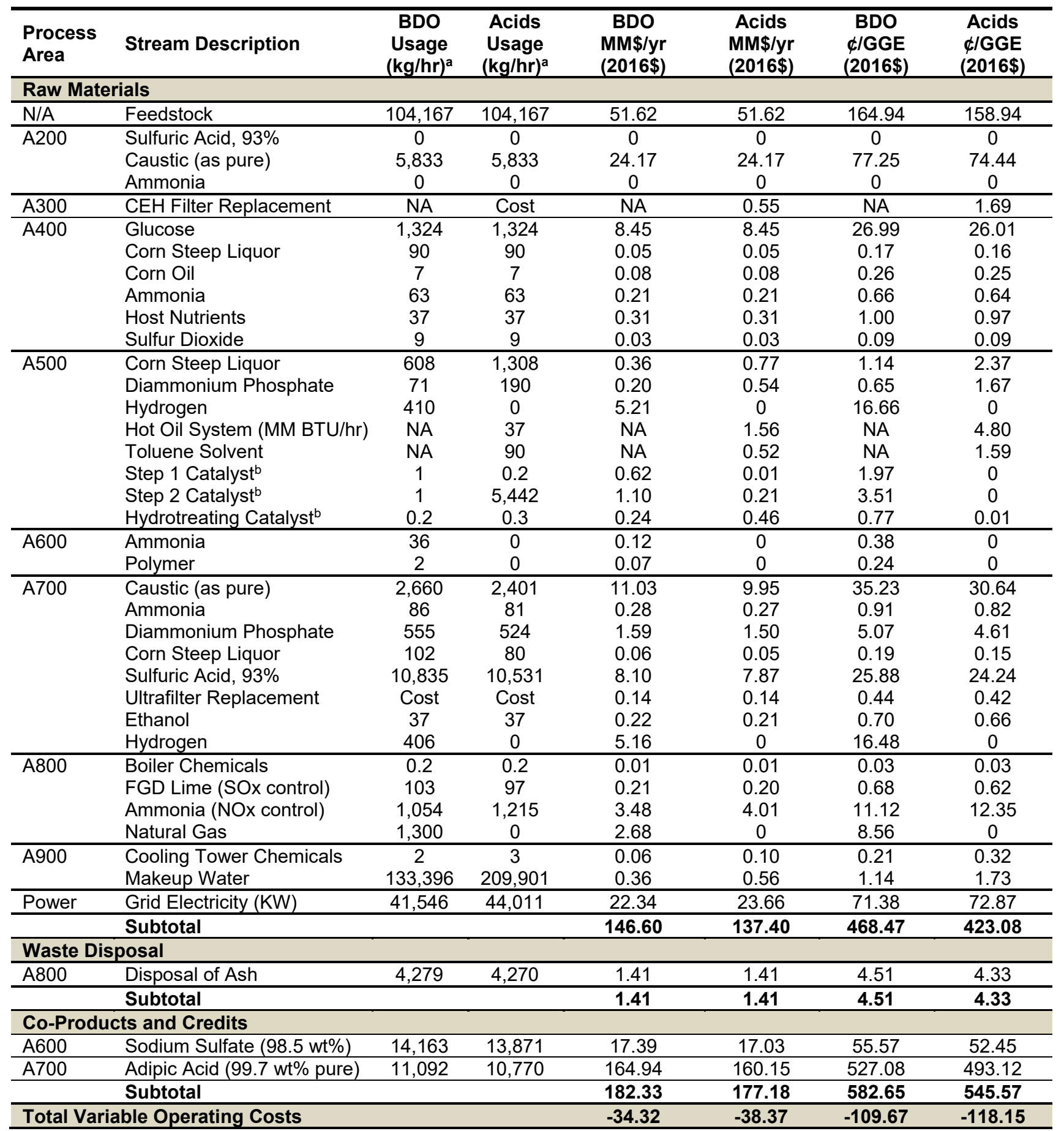

a For reference to convert to kg/GGE basis, fuel outputs are 3,969 and 4,119 GGE/hr for BDO and acids cases respectively.

${ }^{b}$ Catalyst usage amortized to $\mathrm{kg} / \mathrm{hr}$-basis for consistency with rest of table. Step 1 = BDO upgrading (BDO), ketonization

(acids); Step 2 = oligomerization (BDO), condensation (acids); Hydrotreating = hydrogenation (BDO), HDO (acids). 


\subsection{Fixed Operating Costs}

Fixed operating costs are generally incurred in full whether or not the plant is producing at full capacity. These costs include labor and various overhead items. The assumptions on fixed operating costs were generally maintained consistently with the 2011 design basis (after updating to $2016 \$$ as described in Section 4.1), which in turn were based in large part on NREL's 2002 ethanol design report [3] and/or Peters, Timmerhaus, and West [153].

Table 34 shows the recommended number of employees and associated salaries. The number of employees was estimated by considering the likely degree of automation for each area and adding a reasonable number of management and support employees. Details behind the originally assumed number of employees and associated salaries are provided in the 2011 ethanol report. Relative to that basis, some positions were expanded in light of the increased complexity and capital equipment expenditures reflected in the present design; namely plant engineers (4 rather than 2 ) and shift operators (24 rather than 20). Because the model feedstock is predominately corn stover, salaries were estimated for rural regions of the U.S. Midwest. These estimates may vary depending on location. While beyond the scope of this analysis, some economy-of-scale advantages could be gained with respect to labor costs by considering multiple units at the same site rather than a single stand-alone unit, such that some overlapping positions could be shared (e.g., one shared plant manager). However, such savings would likely be marginal in comparison to overall facility costs.

Table 34. Fixed Operating Costs

\begin{tabular}{|c|c|c|c|c|c|c|}
\hline Position & 2016 Salary & \# Required & 2016 Cost & $\begin{array}{l}M M \$ / y r \\
(2016 \$)\end{array}$ & $\begin{array}{c}\text { BDO } \\
\phi / G G E \\
(2016 \$)\end{array}$ & $\begin{array}{l}\text { Acids } \\
\text { \$/GGE } \\
(2016 \$)\end{array}$ \\
\hline \multicolumn{7}{|c|}{ Labor and supervision } \\
\hline Plant Manager & 164,452 & 1 & 164,452 & & & \\
\hline Plant Engineer & 78,310 & 4 & 313,241 & & & \\
\hline Maintenance Supervisor & 63,767 & 1 & 63,767 & & & \\
\hline Maintenance Technician & 44,749 & 12 & 536,985 & & & \\
\hline Lab Manager & 62,648 & 1 & 62,648 & & & \\
\hline Lab Tech & 44,749 & 2 & 89,498 & & & \\
\hline Lab Tech-Enzyme & 44,749 & 2 & 89,498 & & & \\
\hline Shift Supervisor & 53,699 & 4 & 214,794 & & & \\
\hline Shift Operators & 44,749 & 24 & $1,073,970$ & & & \\
\hline Shift Operators-Enzyme & 44,749 & 8 & 357,990 & & & \\
\hline Yard Employees & 31,324 & 4 & 125,297 & & & \\
\hline Clerks and Secretaries & 40,274 & 3 & 120,822 & & & \\
\hline Total Salaries & & & $\$ 3,212,962$ & 3.21 & 10.27 & 9.89 \\
\hline Labor Burden (90\%) & & & $\$ 2,891,655$ & 2.89 & 9.24 & 8.90 \\
\hline Other Overhead & & & $\begin{array}{c}\text { BDO } \\
\mathrm{MM} \$ / \mathrm{yr}\end{array}$ & $\begin{array}{c}\text { Acids } \\
\text { MM } \$ / y r\end{array}$ & $\begin{array}{c}\text { BDO } \\
\text { Cent/GGE }\end{array}$ & $\begin{array}{c}\text { Acids } \\
\text { Cent/GGE }\end{array}$ \\
\hline Maintenance & $3.0 \%$ & of ISBL & 7.95 & 8.82 & 25.42 & 27.17 \\
\hline Property Insurance & $0.7 \%$ & of $\mathrm{FCl}$ & 4.63 & 5.04 & 14.80 & 15.53 \\
\hline \multicolumn{3}{|c|}{ Total Fixed Operating Costs } & 18.69 & 19.97 & 59.73 & 61.50 \\
\hline
\end{tabular}


A $90 \%$ labor burden is applied to the salary total and covers items such as safety, general engineering, general plant maintenance, payroll overhead (including benefits), plant security, janitorial and similar services, phone, light, heat, and plant communications. The $90 \%$ estimate is the median of the general overhead range suggested in the 2008 Process Economics Program Yearbook produced by SRI Consulting [154]. Annual maintenance materials were estimated as 3\% of the installed ISBL capital cost and property insurance, and local property tax was estimated as $0.7 \%$ of the fixed capital investment, based on the 1994 Chem Systems report described in NREL's 2011 ethanol report. These factors are all consistent with those used in the 2011 and 2013 design reports.

\subsection{Discounted Cash Flow Analysis and the Minimum Fuel Selling Price}

\subsubsection{Discount Rate}

For this analysis, the discount rate (which is also the IRR in this analysis) was set to $10 \%$ and the plant lifetime was set to 30 years. The discount rate was also used in previous design reports and was based on the recommendation in Short et al. [155] on how to perform economic evaluations of renewable energy technologies for DOE. His view was that, "In the absence of statistical data on discount rates used by industrial, transportation and commercial investors for investments with risks similar to those of conservation and renewable energy investments, it is recommended that an after tax discount rate of $10 \% \ldots$ be used." The $10 \%$ rate is consistent with all platforms across the BETO portfolio.

\subsubsection{Equity Financing}

Consistent with other recent design reports, it was assumed that the plant would be $40 \%$ equity financed. The terms of the loan were established at $8 \%$ interest for 10 years. The principal is taken out in stages over the 3-year construction period. Interest on the loan is paid during this period, but principal is not paid back (this is another $n^{\text {th }}$-plant assumption, which says that this cash flow comes from the parent company until the plant starts up). This is all consistent with the assumptions used in the 2013 biological hydrocarbon and the 2011 ethanol reports. Figure 19 illustrates the sensitivity of MFSP to the percentage of equity financing and the after-tax discount rate (the IRR). 


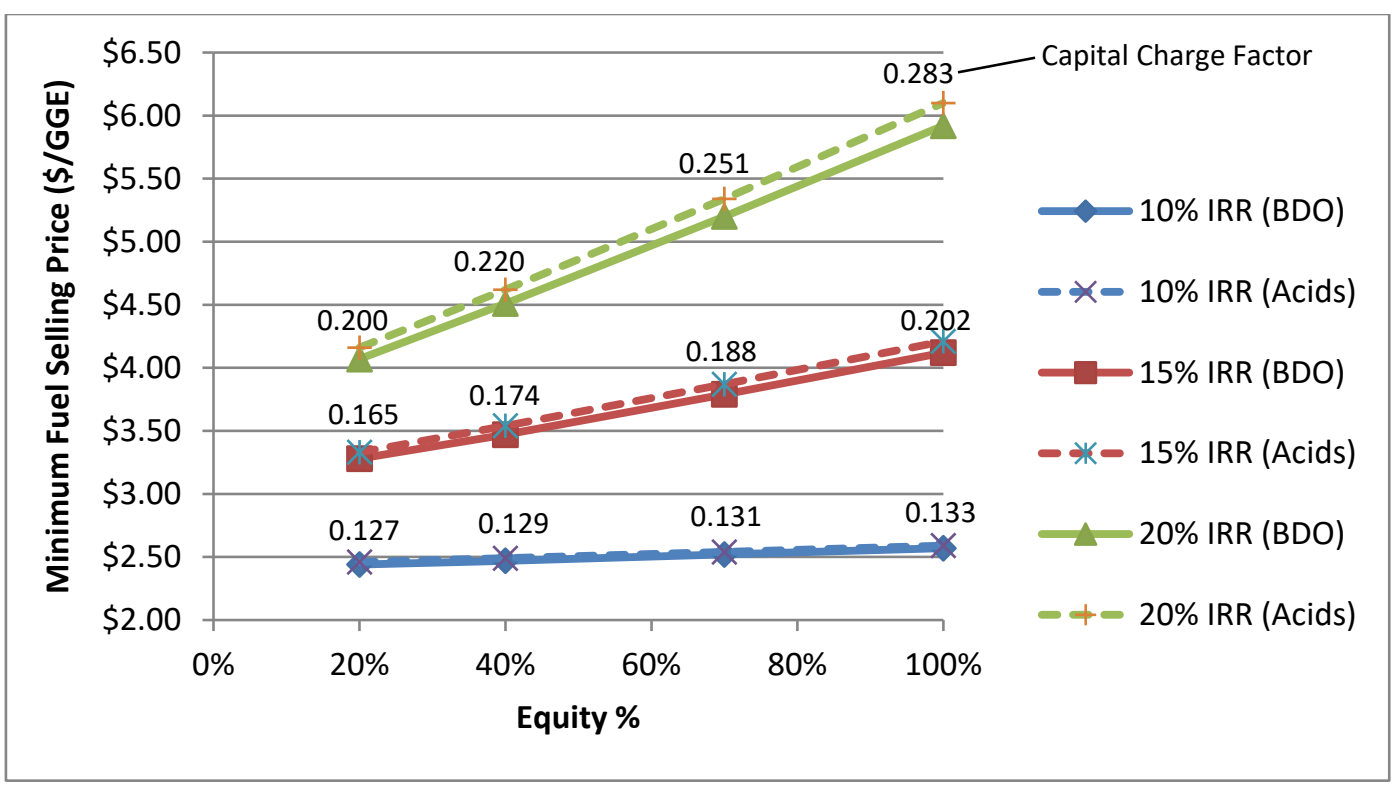

Figure 19. Sensitivity of MFSP to IRR and \% equity for both fuel pathways. ( $8 \%$ interest on a 10-year loan.) Text labels over curves indicate corresponding capital charge factor (similar for BDO and acids cases).

\subsubsection{Other Financial Metrics}

Consistent with prior design cases, the IRS Modified Accelerated Cost Recovery System (MACRS) basis for depreciation schedules is maintained in the present design, which uses a 7-year recovery period for the majority of the plant except for the steam plant equipment (Area 800), which uses a 20year recovery period. More details on depreciation considerations may be found in the 2011 design report. Next, the federal corporate tax rate used in the present analysis has been reduced from $35 \%$ (used in prior design cases) to $21 \%$, reflective of recently passed tax legislation in December 2017 for permanent corporate tax rates. The amount of income tax to be paid by a potential fuel producer varies annually due to changes in the volume of product produced and the allowable depreciation deduction. In fact, no income tax is paid in the first 9 years of operation because the depreciation and loan interest deductions are greater than the net income. State taxes are not considered, primarily because the location of the plant has not been determined and tax rates vary from state to state (from $0 \%$ to $12 \%$ ).

The construction time is important to the cash flow analysis because no income is earned during construction, but large sums of money are being expended. Construction time assumptions were left unchanged from the 2011 and 2013 design basis assumptions. Perry and Green [45] indicate that small projects (less than $\$ 10$ million investment) can be constructed in fewer than 18 months and that larger projects can take up to 42 months. An overview of petroleum refining economics indicates that large refineries (on the order of $\$ 1.5$ billion investment) can be constructed in 24 months [156]. Certainly, this facility is much smaller than a petroleum refinery, so using a construction time of 24 months fits within these references, although an important difference between this type of facility and a refinery is the large number of field-erected vessels. These are constructed on-site and have a longer construction time than if the tanks were delivered finished. Table 35 summarizes the schedule for construction and the cash flow during that time. Twelve months are added before construction for planning and engineering. 
Table 35. Construction Activities and Cash Flow

\begin{tabular}{|c|c|c|c|}
\hline $\begin{array}{l}\text { Project } \\
\text { Start } \\
\text { Month }\end{array}$ & $\begin{array}{l}\text { Project } \\
\text { End } \\
\text { Month }\end{array}$ & Activity Description & $\begin{array}{l}\% \text { of } \\
\text { Project } \\
\text { Cost }\end{array}$ \\
\hline 0 & 12 & $\begin{array}{l}\text { Project plan and schedule established; conceptual and basic design } \\
\text { engineering and permitting completed; major equipment bid packages } \\
\text { issued; engineering started on selected sub-packages; P\&IDs } \\
\text { complete; and preliminary plant and equipment arrangements } \\
\text { complete. }\end{array}$ & $8 \%$ \\
\hline 12 & 24 & $\begin{array}{l}\text { All detailed engineering-including foundations, structure, piping, } \\
\text { electrical, and site-complete; all equipment and instrument } \\
\text { components purchased and delivered; all site grading, drainage, } \\
\text { sewers, rail, fire pond, foundation, and major structural installation } \\
\text { complete; } 80 \% \text { of all major process equipment set (all except longest- } \\
\text { lead items), all field fabricated tanks built; and the majority of piping } \\
\text { and electrical materials procured. }\end{array}$ & $60 \%$ \\
\hline \multirow[t]{2}{*}{24} & 36 & $\begin{array}{l}\text { Complete process equipment setting, piping, and instrumentation } \\
\text { installation complete; all electrical wiring complete; all building finishing } \\
\text { and plumbing complete; all landscaping complete; pre-commissioning } \\
\text { complete; and commissioning, start-up, and initial performance test } \\
\text { complete. }\end{array}$ & $32 \%$ \\
\hline & & TOTAL & $100 \%$ \\
\hline
\end{tabular}

Similar to 2013 design basis, a start-up time of 6 months under an $n^{\text {th }}$-plant assumption was assumed in this analysis. Perry and Green [45] indicate that for a moderately complex plant, startup should be about $25 \%$ of the construction time, or 6 months in this case. The start-up period is not completely wasted, however. We expect that an average of $50 \%$ production could be achieved during that period while incurring $75 \%$ of variable expenses and $100 \%$ of fixed expenses. Finally, the present analysis applies the same basis for working capital as was used in the 2011 ethanol report, namely $5 \%$ of FCI. Peters, Timmerhaus, and West [153] define working capital as money available to cover (a) raw materials and supplies in inventory, (b) finished product in storage, (c) accounts receivable, (d) cash on hand for monthly payments such as wages and maintenance supplies, (e) accounts payable, and (f) taxes payable.

\subsubsection{Base Case TEA Results}

Table 36 summarizes the parameters used in the discounted cash flow analysis. Using these parameters, plus the cost information in Table 31, Table 33, and Table 34, the resulting MFSP of total fuel products is \$2.47/GGE for the BDO case and \$2.49/GGE for the acids case (2016\$), including all fuel-range product cuts from distillation adjusted by heating values (calculated in the Aspen model) to gasoline equivalents.

Table 37 summarizes the yields and conversion costs for the present designs. According to the methodology of Cran [17], the expected accuracy of the overall TCI analysis is $\pm 25 \%$ (although some specific pieces of equipment carry a higher degree of uncertainty in underlying cost estimates as identified above). If we apply this uncertainty to the TCI, the impact on the cost of total fuel is $\pm \$ 0.73 / \mathrm{GGE}$ and $\$ 0.77 / \mathrm{GGE}$ for the BDO and acids cases, respectively. The complete discounted cash flow summary worksheets are shown in Appendix B. The MFSP can be further broken down into the 
cost of each process area. Figure 20 and Figure 21 illustrate the contribution to the overall cost by process area and capital, operations, and fixed costs (the bar for feedstock plus handling reflects the single feedstock cost of $\$ 71.26$ /dry U.S. tons delivered to pretreatment and has not been broken down).

Table 36. Discounted Cash Flow Analysis Parameters

\begin{tabular}{ll}
\hline Plant life & 30 years \\
Discount rate & $10 \%$ \\
General plant depreciation & $200 \%$ declining balance (DB) \\
General plant recovery period & 7 years \\
Steam plant depreciation & $150 \%$ DB \\
Steam plant recovery period & 20 years \\
Federal tax rate & $21 \%$ \\
Financing & $40 \%$ equity \\
Loan terms & 10 -year loan at $8 \%$ APR \\
Construction period & 3 years \\
First 12 months' expenditures & $8 \%$ \\
Next 12 months' expenditures & $60 \%$ \\
Last 12 months' expenditures & $32 \%$ \\
Working capital & $5 \%$ of fixed capital investment \\
Start-up time & 6 months \\
Revenues during startup & $50 \%$ \\
Variable costs incurred during startup & $75 \%$ \\
Fixed costs incurred during startup & $100 \%$ \\
\hline
\end{tabular}

Table 37. Summary of Yields, Rates, and Conversion Costs for Both Base Cases

\begin{tabular}{|c|c|c|}
\hline & BDO Pathway & Acids Pathway \\
\hline Feedstock rate & \multicolumn{2}{|c|}{2,205 dry U.S. tons/day } \\
\hline Online time & \multicolumn{2}{|c|}{$7,884 \mathrm{~h} / \mathrm{yr}$ (90\% online factor) } \\
\hline Total fuel yield & $\begin{array}{l}\text { 43.2 GGE/dry U.S. ton } \\
\text { feedstock }\end{array}$ & $\begin{array}{c}\text { 44.8 GGE/dry U.S. ton } \\
\text { feedstock }\end{array}$ \\
\hline Total fuel production rate & 31.3 MM GGE/yr & 32.5 MM GGE/yr \\
\hline Diesel-range production rate & 16.4 MM GGE/yr & 32.5 MM GGE/yr \\
\hline Naphtha-range production rate & 14.9 MM GGE/yr & - \\
\hline Adipic acid coproduct yield & $266 \mathrm{lb} / \mathrm{dry}$ U.S. ton feedstock & $259 \mathrm{lb} / \mathrm{dry}$ U.S. ton feedstock \\
\hline Adipic acid production rate & $193 \mathrm{MM} \mathrm{lb/yr}$ & $187 \mathrm{MM}$ Ib/yr \\
\hline Total variable opex excluding coproduct ${ }^{a}$ & $\$ 148 \mathrm{MM} / \mathrm{yr}$ & $\$ 139 \mathrm{MM} / \mathrm{yr}$ \\
\hline Coproduct revenue & $\$ 182 \mathrm{MM} / \mathrm{yr}$ & $\$ 177 \mathrm{MM} / \mathrm{yr}$ \\
\hline Total fixed opex & $\$ 19 \mathrm{MM} / \mathrm{yr}$ & $\$ 20 \mathrm{MM} / \mathrm{yr}$ \\
\hline Total equipment cost & $\$ 367 \mathrm{MM}$ & $\$ 399$ MM \\
\hline Total capital investment (TCl) & $\$ 697 \mathrm{MM}$ & $\$ 758 \mathrm{MM}$ \\
\hline $\mathrm{TCl}$ per annual gallon & $\$ 22.27 / G G E$ & $\$ 23.35 / G G E$ \\
\hline Minimum Fuel Selling Price & $\$ 2.47 / G G E$ & $\$ 2.49 / G G E$ \\
\hline Feedstock contribution & $\$ 1.65 / G G E$ & $\$ 1.59 / G G E$ \\
\hline Fuel conversion contribution & $\$ 3.83 / \mathrm{GGE}$ & $\$ 3.87 / G G E$ \\
\hline Coproduct conversion contribution & $-\$ 3.00 / G G E$ & $-\$ 2.97 /$ GGE \\
\hline
\end{tabular}

${ }^{a}$ Excludes coproduct revenue from sale of adipic acid and sodium sulfate (next row). Not including catalyst replacement schedules. 

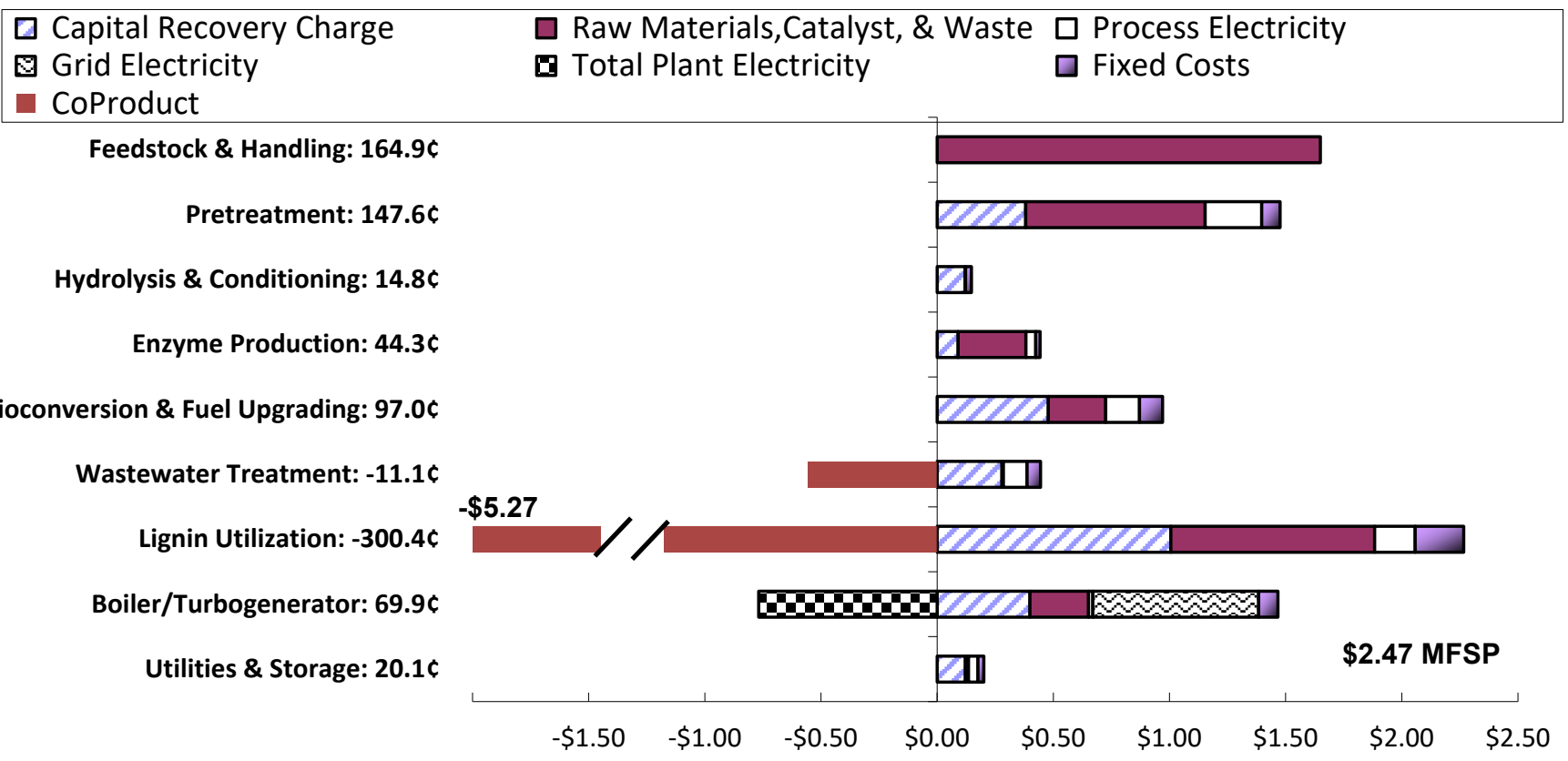

Figure 20. BDO pathway cost contribution details from each process area (per GGE total fuel products)

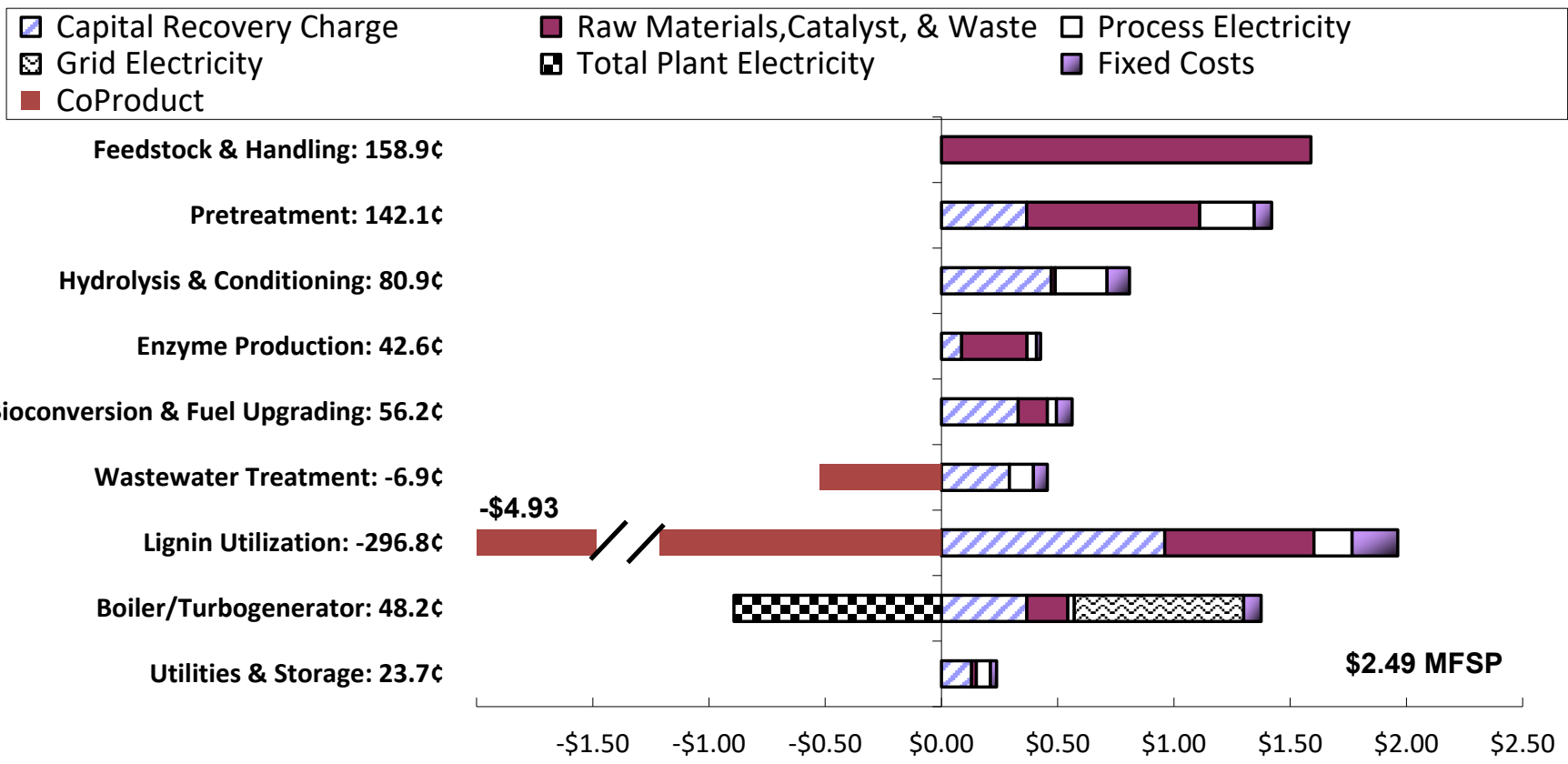

Figure 21. Acids pathway cost contribution details from each process area (per GGE total fuel products)

As shown in Table 37, Figure 20, and Figure 21, the MFSP estimates for both the BDO and acids pathways are seen to be quite comparable, with the BDO pathway exhibiting a roughly $3 \%$ lower yield (GGE/ton) but offset by a roughly $8 \%$ lower TCI. Variable operating costs for the BDO pathway are marginally higher than the acids pathway, primarily driven by a larger hydrogen deficit, due to catalytic upgrading demands versus hydrogen co-produced from fermentation. However, they are 
counterbalanced by higher adipic acid coproduct yield/revenue, due to more unconverted sugars and other convertible components reaching the lignin train with the placement of solid separation downstream of BDO fermentation, and slightly lower fixed operating costs (partially tied to capital costs).

As is typical for TEA models, feedstock constitutes the largest MFSP contribution at roughly $\$ 1.60$ $\$ 1.65 / \mathrm{GGE}$, followed by pretreatment (DMR operations, including substantial costs for caustic demands) at nearly $\$ 1.50 / \mathrm{GGE}$ and then either bioconversion and upgrading (BDO case) or hydrolysis and conditioning (acids case) at \$0.97/GGE and \$0.81/GGE for BDO and acids respectively. Also consistent with recent TEA models since 2013, enzyme contributions constitute one of the smaller cost drivers at the targeted $10 \mathrm{mg} / \mathrm{g}$ enzyme loading (this can be substantially higher at increased enzyme loading demands).

Comparing cost breakdowns in individual process areas, the acids pathway incurs a considerably larger cost for Area 300 (enzymatic hydrolysis and conditioning) on the order of $\$ 0.66 / \mathrm{GGE}$, due to more complex CEH processing than batch EH (although this includes the integrated solids removal steps which are required regardless for the acids pathway), as well as the inclusion of the hydrolysate evaporator. This is partially offset by lower costs for Area 500 (bioconversion and fuel upgrading) and 800 (CHP system). Area 500 costs contribute roughly $\$ 0.41 /$ GGE less for acids than BDO, primarily due to less costly catalytic upgrading steps designed to process only the acid intermediate (albeit at the added expense of pertraction membranes to first isolate the acid), versus the BDO pathway, which must process a much larger aqueous stream with roughly $90 \%$ water through the first BDO upgrading reactor. Indeed, the catalytic reaction steps taken in isolation are estimated to cost roughly \$36 MM for the $\mathrm{BDO}$ case versus $\$ 21 \mathrm{MM}$ for the acids case (installed equipment costs). Area 800 contributes roughly $\$ 0.22 /$ GGE less for acids than BDO, primarily due to lower biorefinery heat demands, which is driven in turn by catalytic upgrading that requires more heat for the BDO case given the much larger aqueous throughputs through the initial $\mathrm{BDO}$ upgrading step. Therefore, it is necessary to send more available steam through the turbine to generate more power. The differences between the other process area contributions generally reflect the fuel (GGE) and coproduct yield differences respectively. It is also worth noting that the wastewater treatment area now translates to a small net negative MFSP contribution after removing anaerobic digestion (typically the largest WWT cost driver) and including the sale of sodium sulfate coproduct. 


\section{Analysis and Discussion}

\subsection{Carbon, Water, and Energy Balances}

Table 38 shows the overall flow of carbon inputs and outputs, with a carbon balance closure very near unity. As shown in the table, $>96 \%$ of all carbon in the process enters in the biomass feed, with a small amount additional carbon primarily coming from glucose (for enzyme production) and natural gas (combusted in the boiler for supplemental heat in the BDO case). Bioconversion nutrients like CSL are a very minor contributor to the carbon balance. For the most part, fermentor vents are routed to the boiler in Area 800 and are reflected as flue gas. In both cases, roughly $40 \%$ of the carbon input leaves as fuels and coproducts, allocated roughly $65 \%$ to fuels and $35 \%$ to coproducts. The carbon yield to fuels at $24 \%-26 \%$ is similar (slightly lower) compared to prior biological pathway models i.e. the 2011 ethanol and 2013 hydrocarbon design reports at $29 \%$ and $26 \%$, respectively, but with the addition of coproducts derived from lignin, extractives, and other residual carbon components adding another $14 \%$, the resulting overall biorefinery carbon yield to "useful" products is now substantially improved. Major exit points for the balance of carbon are the combustor stack and the aerobic digestion lagoons.

Table 38. Biorefinery Overall Carbon Balance

\begin{tabular}{|c|c|c|c|c|}
\hline \multirow[b]{2}{*}{ Stream } & \multicolumn{2}{|c|}{ Acids Case } & \multicolumn{2}{|c|}{ BDO Case } \\
\hline & $\begin{array}{c}\text { Carbon Flow } \\
(\mathrm{kmol} / \mathrm{h})\end{array}$ & $\begin{array}{c}\% \text { of Carbon } \\
\text { Flow }\end{array}$ & $\begin{array}{c}\text { Carbon Flow } \\
(\mathrm{kmol} / \mathrm{h})\end{array}$ & $\begin{array}{c}\text { \% of Carbon } \\
\text { Flow }\end{array}$ \\
\hline \multicolumn{5}{|l|}{ Carbon inlets } \\
\hline Biomass feedstock & 3,084 & $97 \%$ & 3,084 & $96 \%$ \\
\hline Natural gas & - & - & 81 & $2 \%$ \\
\hline Glucose & 44 & $1 \%$ & 44 & $1 \%$ \\
\hline Chemical inputs & 40 & $1 \%$ & 20 & $1 \%$ \\
\hline Total & 3,168 & $100 \%$ & 3,230 & $100 \%$ \\
\hline \multicolumn{5}{|l|}{ Carbon outlets } \\
\hline Area 500 fuel output & 808 & $26 \%$ & 770 & $24 \%$ \\
\hline Area 700 coproduct & 443 & $14 \%$ & 457 & $14 \%$ \\
\hline Combustor flue gas & 1,770 & $56 \%$ & 1,796 & $56 \%$ \\
\hline Aerobic lagoons & 144 & $5 \%$ & 214 & $7 \%$ \\
\hline Total & 3,164 & $100 \%$ & 3,238 & $100 \%$ \\
\hline
\end{tabular}

The overall flow of water throughout the model is presented in Table 39 and Table 40. Cooling tower evaporation accounts for the majority of the total process makeup water demand. Between the two pathways, the BDO case translates to a lower process makeup water demand at 8.9 versus 13.5 gal/GGE (and lower overall water "input" of 13.6 versus $17.4 \mathrm{gal} / \mathrm{GGE}$ ). This is due primarily to higher TS concentrations through enzymatic hydrolysis and fermentation (i.e., batch EH requires less dilution water than $\mathrm{CEH}$ ). As discussed above, lower A800 excess steam availability and thus condensing steam turbine cooling demands in the BDO case also contribute to the differences in makeup water demand. 
Table 39. Biorefinery Water Balance, Acids Case

\begin{tabular}{lcc|lcc}
\hline Water Balance & & & & \\
\hline Inputs & $\mathbf{k g} / \mathbf{h r}$ & $\mathbf{g a l} / \mathbf{G G E}$ & Outputs & $\mathbf{k g} / \mathbf{h r}$ & $\mathbf{g a l} / \mathbf{G G E}$ \\
Moisture in feedstock & 20,833 & 1.3 & Cooling tower losses & 195,823 & 12.6 \\
Water in glucose syrup & 234 & 0.0 & Fermentor vents & 424 & 0.0 \\
Water in raw chemicals & 1,627 & 0.1 & WWT evaporation & 21,968 & 1.4 \\
Net reaction water & 28,483 & 1.8 & WWT discharge & 158 & 0.0 \\
Air intake moisture & 9,611 & 0.6 & Boiler blowdown vent & 1,558 & 0.1 \\
Makeup water & 209,901 & 13.5 & Flue gas & 50,737 & 3.3 \\
& & & Water in product & 21 & 0.0 \\
Sum of Inputs & $\mathbf{2 7 0 , 6 8 9}$ & $\mathbf{1 7 . 4}$ & Sum of Outputs & $\mathbf{2 7 0 , 6 8 8}$ & $\mathbf{1 7 . 4}$ \\
\hline
\end{tabular}

Table 40. Biorefinery Water Balance, BDO Case

\begin{tabular}{lcc|lcc}
\hline Water Balance & & & & \\
\hline Inputs & $\mathbf{k g} / \mathbf{h r}$ & $\mathbf{g a l} / \mathbf{G G E}$ & Outputs & $\mathbf{k g} \mathbf{h r}$ & $\mathbf{g a l} / \mathbf{G G E}$ \\
Moisture in feedstock & 20,833 & 1.4 & Cooling tower losses & 121,177 & 8.1 \\
Water in glucose syrup & 234 & 0.0 & Fermentor vents & 424 & 0.0 \\
Water in raw chemicals & 1,308 & 0.1 & WWT evaporation & 22.096 & 1.5 \\
Net reaction water & 37,894 & 2.5 & WWT brine & 87 & 0.0 \\
Air intake moisture & 10,457 & 0.7 & Boiler blowdown vent & 1,950 & 0.1 \\
Makeup water & 133,396 & 8.9 & Flue gas & 58,324 & 3.9 \\
Sum of Inputs & $\mathbf{2 0 4 , 1 2 1}$ & $\mathbf{1 3 . 6}$ & Sum of Outputs & $\mathbf{2 4 3 , 0 0 0}$ & $\mathbf{1 3 . 6}$ \\
\hline
\end{tabular}

Finally, Figure 22 presents energy balance allocations for all energy outputs embodied in each major exit point from the biorefinery. Energy in the fuel product, adipic acid coproduct, heat rejection to the cooling tower, and out the flue gas stack constitute the primary energy output flows. Energy outputs in all "useful" products (i.e. the fuel and coproduct combined) represent between $39-43 \%$ of total biorefinery outputs, while "waste" heat rejection from cooling water and air cooler utilities constitutes $30-33 \%$ across the two pathways. It is worth noting here that relative to prior biochemical design cases, the present design makes significantly more use of both biological (low-temperature) and catalytic (high-temperature) processing steps, given the catalytic upgrading train required for both pathways after sugar fermentation to fuel precursors, as well as elevated temperatures for lignin deconstruction and coproduct upgrading. While efforts were made to optimize process heat integration, further room for better optimization may still exist to reduce waste heat losses through more rigorous pinch analysis, as an area for further refinement moving forward. Even so, energy losses to "waste" outputs other than the primary products are always inevitable in any integrated process such as this.

It should also be noted that the overall energy flows are derived from inputs beyond just the biomass feed. Table 41 shows a high-level energy balance of all energy input and output flows. Biomass feedstock constitutes $349 \mathrm{MMkcal} / \mathrm{hr}$, which represents $84 \%$ of all energy inputs for the acids case (416 MMkcal $/ \mathrm{hr}$ total) or $78 \%$ of all inputs for the BDO case (449 MMkcal $/ \mathrm{hr}$ total). Imported power, natural gas, and raw chemicals also carry considerable embodied energy input flows for both pathways. Total energy inputs are higher for the BDO pathway given more natural gas heating demands as well as a hydrogen deficit which requires importing additional hydrogen. 
(a)

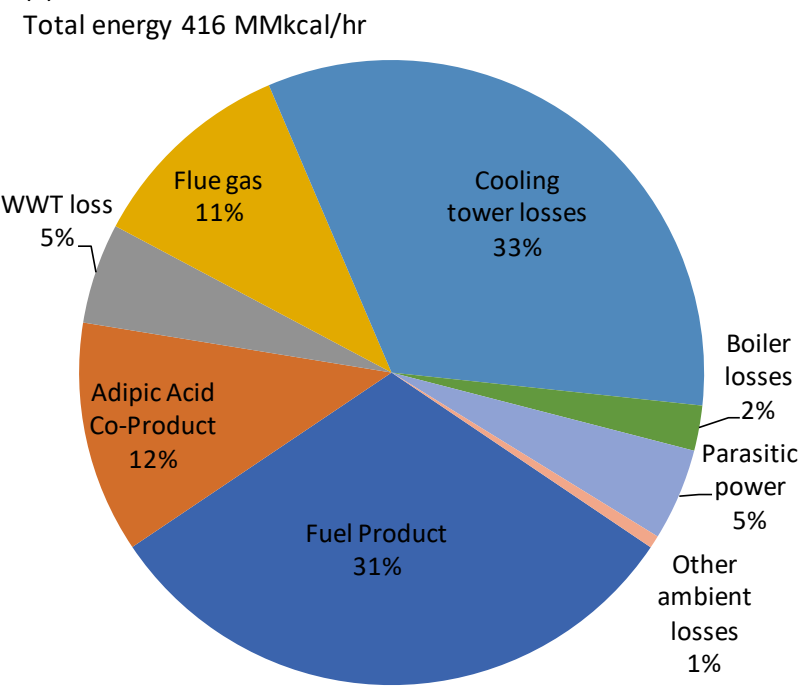

(b)

Total energy $449 \mathrm{MMkcal} / \mathrm{hr}$

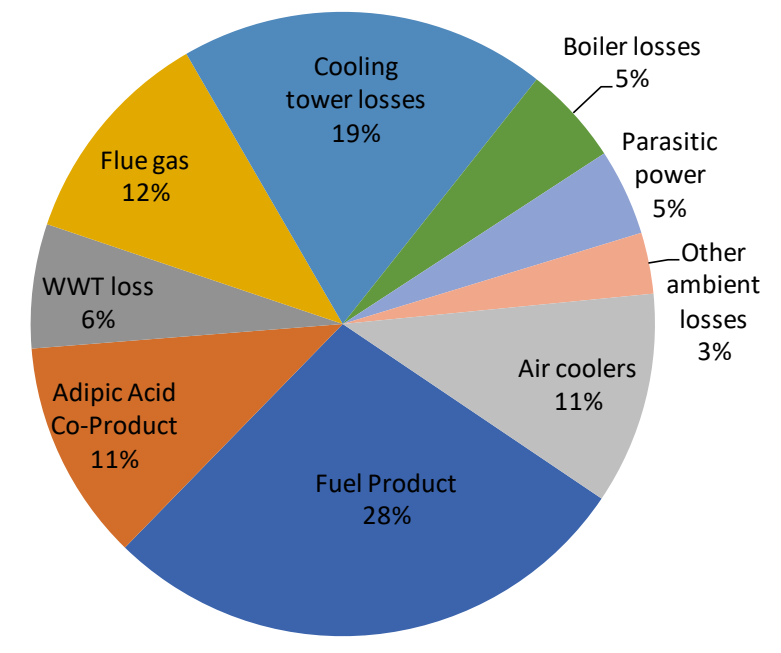

Figure 22. Distribution of energy outputs embodied in major exit points; (a) acids case, (b) BDO case

Table 41. Overall Input/Output Energy Balances

\begin{tabular}{|lcc|lcc|}
\hline Inputs (MMkcal/hr) & Acids & BDO & Outputs (MMkcal/hr) & Acids & BDO \\
\hline Feedstock & 349 & 349 & Fuel Product & 130 & 125 \\
Glucose Syrup & 5 & 5 & Adipic Acid Co-Product & 50 & 51 \\
Raw chemicals & 12 & 8 & WWT losses b & 22 & 28 \\
Fresh water ${ }^{\text {a }}$ & -2 & -1 & Flue gas & 45 & 51 \\
Air & 6 & 6 & Cooling tower losses & 138 & 85 \\
Imported power & 39 & 36 & Boiler losses & 10 & 23 \\
Natural gas & 8 & 17 & Air Cooler & - & 49 \\
Hydrogen & 0 & 28 & Parasitic power & 20 & 20 \\
& & & Other ambient losses & 3 & 14 \\
\hline Sum of Inputs & $\mathbf{4 1 6}$ & $\mathbf{4 4 9}$ & Sum of Outputs & $\mathbf{4 1 6}$ & $\mathbf{4 4 9}$ \\
\hline
\end{tabular}

a Value appears negative due to selected reference states for calculating enthalpy flows.

b Includes energy outputs in WWT brine plus aerobic lagoon evaporation

\subsection{Cost Sensitivity Analysis}

For each of the pathways described previously in the report, the techno-economic models were used to perform sensitivity analysis on key model variables. Beginning with the baseline for each variable as described in this report, minima and maxima were chosen based on reasonable limits surrounding the specific process, conversations with researchers, and expected error margins on capital costs. Each variable was changed to its maxima/minima with all other factors held constant. The resulting model outputs are used to help understand and quantify cost impacts on the overall MFSP. 


\subsubsection{Single-Point Sensitivity Analysis: Acids to Fuels Pathway}

For the acids pathway, key parameters for pretreatment, hydrolysis, fermentation, and upgrading, as well as lignin utilization, were explored. Table 42 lists the studied variables, their baseline values, and the associated minima/maxima. The sensitivities of (a) MFSP and (b) fuel product yield are displayed as tornado charts in Figure 23. For the yield studies, not all variables showed a significant effect and only a subset of the metrics used in Table 42 are included in the tornado chart.

The uncertainty in capital cost associated with the factored approach used in this study $( \pm 25 \% \mathrm{TCI}$ ) shows the largest impact on MFSP and is more pronounced compared to previous designs due to the increase in overall capital associated with an additional lignin processing train. Beyond capital costs, the amount of lignin accessible to the muconate fermentation (or viewed alternatively, the net overall conversion potential to muconic acid from original lignin in the delivered biomass inclusive of BCD deconstruction and bioconversion) and the muconic acid fermentation productivity have the next largest impacts on MFSP, i.e. lignin train conversion performance reflects the two largest single process-related sensitivity parameters. As a simplistic estimate for what might be required to achieve more near-term $\$ 3 /$ GGE MFSP goals by 2022 as an interim benchmark on the path to $<\$ 2.50 /$ GGE by 2030 , if all other target conversion parameters remained unchanged, overall conversion efficiency of biomass lignin to adipic acid could be relaxed by roughly $12 \%$ from 259 to $229 \mathrm{lb} /$ dry ton, inclusive of lignin deconstruction and conversion yields. This is assuming that many other parameters could also be achieved by 2022 , for example in the fuel train and downstream lignin upgrading steps. This is also inclusive of a higher interim 2022 biomass feedstock cost projection at $\$ 79.07 /$ dry ton (versus the final 2030 target cost of $\$ 71.26 /$ dry ton) based on inputs from INL, translating to an interim MFSP of $\$ 3.02 / \mathrm{GGE}$.

For the fuel train parameters, the butyric acid separation efficiency has the largest effect on fuel yield and a correspondingly large impact on MFSP. Following acid product recovery, the CEH solids loading also reflects a major MFSP impact, particularly if solids loading dropped from $7.5 \%$ to $5 \%$ given substantial costs for re-concentrating the clarified sugars. Net conversion of sugars to products during fermentation has a large effect on overall fuel yield which includes glucose, xylose, and arabinose contributions, but is also affected by the amount of contamination to non-acid products that occurs. We note here that operating parameters varied in the underlying process model have impacts on multiple economic contributors including product yield, capital costs, and operating costs, and more properly represent risks or alternatively represent a range of results that are possible depending on the operation and success of the bench- and pilot-scale campaigns. In comparison, varying a parameter like the TCI $\pm 25 \%$ represents a true uncertainty surrounding the modeling methodology or price estimates. 
Table 42. Assumptions Varied in the Acids Sensitivity Analysis

\begin{tabular}{|c|c|c|c|c|}
\hline & Assumption & Min MFSP & Baseline & Max MFSP \\
\hline \multirow[t]{3}{*}{ Pretreatment } & DMR NaOH loading $(\mathrm{mg} / \mathrm{g})$ & 50 & 70 & 100 \\
\hline & DMR xylan to liquor (wt\%) & $25.0 \%$ & $10.0 \%$ & $5.0 \%$ \\
\hline & DMR lignin to liquor (wt\%) & $60.0 \%$ & $47.0 \%$ & $25.0 \%$ \\
\hline \multirow{6}{*}{$\begin{array}{l}\text { Continuous } \\
\text { Enzymatic } \\
\text { Hydrolysis }\end{array}$} & CEH cellulose to glucose (wt\%) & $99.0 \%$ & $96.3 \%$ & $90.0 \%$ \\
\hline & CEH xylan to xylose (wt\%) & $99.0 \%$ & $98.9 \%$ & $90.0 \%$ \\
\hline & CEH Solids loading (wt\%) & $10.0 \%$ & $7.5 \%$ & $5.0 \%$ \\
\hline & CEH ultrafilter permeance & 80 & 66.66 & 50 \\
\hline & CEH microfiltration permeance & 115 & 99.99 & 85 \\
\hline & Flocculant Requirement (g/kg Solid) & - & 0 & 20 \\
\hline \multirow{17}{*}{$\begin{array}{l}\text { Enzyme } \\
\text { Production } \\
\text { Acid Fermentation } \\
\text { and Catalysis }\end{array}$} & Enzyme production capital cost & $-50 \%$ & - & $+50 \%$ \\
\hline & Enzyme Licensing & - & 0 & 0.1 \\
\hline & Fermentation contamination loss (wt\%) & $0 \%$ & $3 \%$ & $6 \%$ \\
\hline & Fermentation glucose to product (wt\%) & $100 \%$ & $95 \%$ & $85 \%$ \\
\hline & Fermentation xylose to product (wt\%) & $90 \%$ & $85 \%$ & $75 \%$ \\
\hline & Fermentation arabinose to product (wt $\%)$ & $90 \%$ & $85 \%$ & $75 \%$ \\
\hline & Fermentation Productivity (g/L/hr) & 2.5 & 2 & 1.5 \\
\hline & Fermentation bioreactor volume (gal) & - & $1 \mathrm{MM}$ gal & $0.2 \mathrm{MM}$ gal \\
\hline & Pertraction butyric acid recovery (wt\%) & - & 1 & 0.85 \\
\hline & Condensation solvent:ketone ratio (w/w) & 5 & 3.64 & 1 \\
\hline & Condensation single pass conversion & $90 \%$ & $60 \%$ & $40 \%$ \\
\hline & Fermentation pertraction membrane cost & $-50 \%$ & - & $150 \%$ \\
\hline & Acid ketonization WHSV (hr-1) & 10 & 6 & 2 \\
\hline & Condensation residence time (hr) & 5 & 15 & 24 \\
\hline & Enone hydrogenation WHSV (hr-1) & 10 & 3 & 1 \\
\hline & Enone hydrogenation Platinum loading (wt \%) & $1 \%$ & $3 \%$ & - \\
\hline & Acid catalysis train capital cost & $-50 \%$ & - & $150 \%$ \\
\hline \multirow[t]{7}{*}{ Lignin Utilization } & Muconic metabolic yield (g/g sugar) & 0.408 & 0.3076 & - \\
\hline & Muconic productivity (g/L/hr) & 2 & 1 & 0.5 \\
\hline & Muconic acid fermentation $\mathrm{pH}$ control & none & controlled & - \\
\hline & Muconic acid hydrogenation Temperature $\left({ }^{\circ} \mathrm{C}\right)$ & 100 & 78 & - \\
\hline & Sugar diversion to coproduct (wt $\%$ clean sugar) & $10 \%$ & $0 \%$ & - \\
\hline & Metabolically accessible lignin (wt\%) & 0.675 & 0.5333 & 0.405 \\
\hline & Oslo Crystallizer capital cost & $-20.0 \%$ & - & $20.0 \%$ \\
\hline \multirow[t]{2}{*}{ Economics } & Total capital investment $(\mathrm{TCl})$ & $-25.0 \%$ & - & $25.0 \%$ \\
\hline & Feedstock Cost (\$/dry ton) & 60 & 71.26 & 80 \\
\hline
\end{tabular}


Total capital investment ( $\mathrm{TCl}$ )

Metabolically accessible lignin (wt\%)

Muconic productivity ( $\mathrm{g} / \mathrm{L} / \mathrm{hr}$ )

Pertraction butyric acid recovery (wt\%)

CEH Solids loading (wt\%)

DMR NaOH loading $(\mathrm{mg} / \mathrm{g})$

Feedstock Cost (\$/dry ton)

DMR xylan to liquor (wt\%)

Sugar diversion to coproduct (wt\% clean sugar)

Fermentation glucose to product (wt\%)

Flocculant Requirement (g/kg Solid)

Muconic metabolic yield ( $\mathrm{g} / \mathrm{g}$ sugar)

Muconic acid hydrogenation Temperature $\left({ }^{\circ} \mathrm{C}\right)$

Condensation solvent:ketone ratio $(\mathrm{w} / \mathrm{w})$

Fermentation xylose to product (wt\%)

Acid catalysis train capital cost

DMR lignin to liquor ( $w t \%$ )

Condensation single pass conversion

Muconic acid fermentation $\mathrm{pH}$ control

Enone hydrogenation WHSV (hr-1)

Enzyme production capital cost

Fermentation bioreactor volume (gal)

Fermentation pertraction membrane cost

CEH ultrafilter permeance

Enzyme Licensing

Condensation residence time (hr)

Oslo Crystallizer capital cost

Fermentation Productivity (g/L/hr)

CEH microfiltration permeance

CEH cellulose to glucose (wt\%)

Fermentation arabinose to product (wt\%)

CEH xylan to xylose (wt\%)

Acid ketonization WHSV (hr-1)
Fermentation contamination loss (wt\%)

Enone hydrogenation Platinum loading (wt\%)

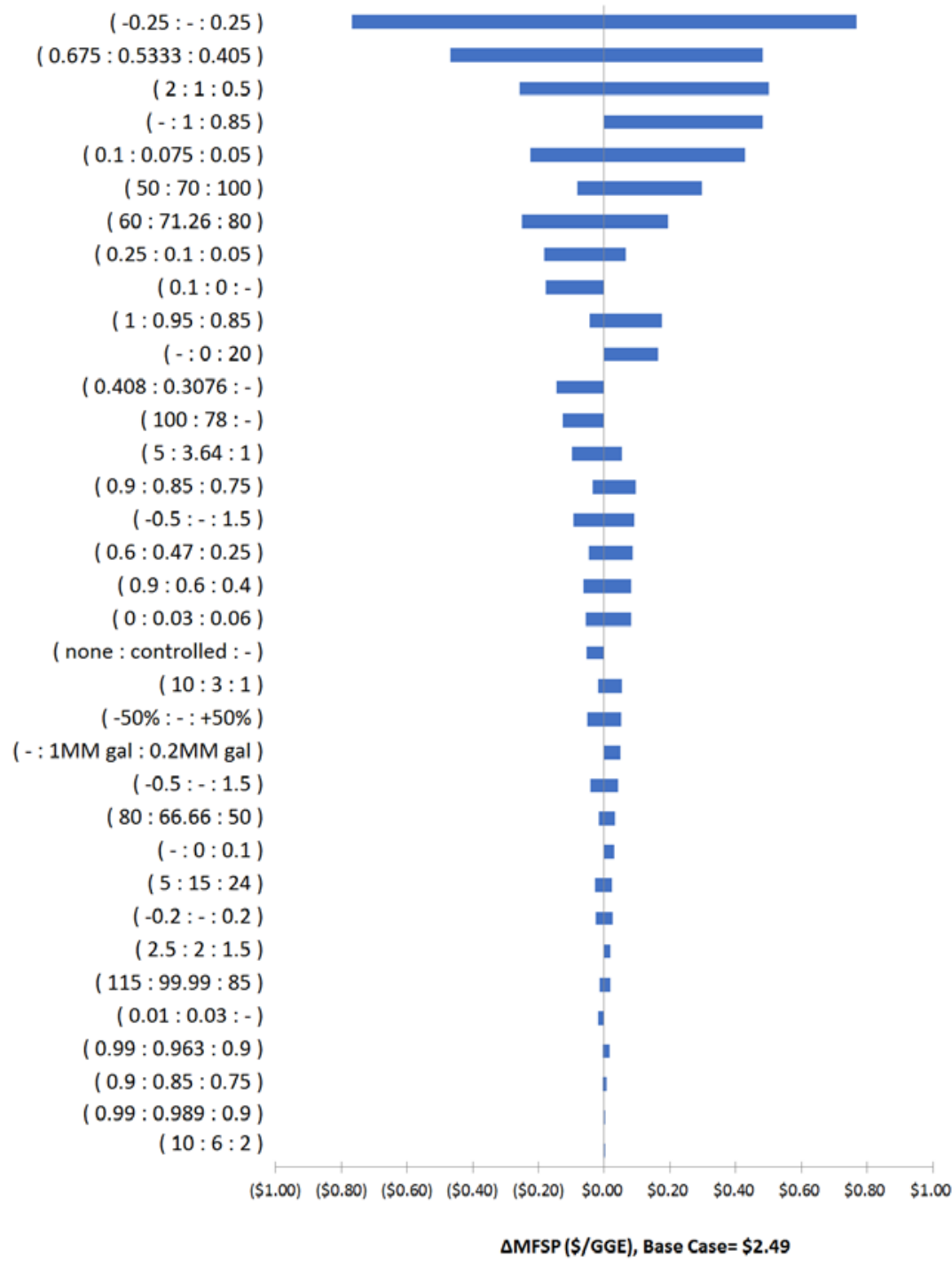

$\triangle \mathrm{MFSP}(\mathbf{\$ G G E})$, Base Case $=\mathbf{\$ 2 . 4 9}$

Pertraction butyric acid recovery (wt\%)

Sugar diversion to coproduct ( $w t \%$ clean sugar)

Fermentation glucose to product ( $w t \%)$

DMR xylan to liquor (wt\%)

CEH cellulose to glucose ( $w t \%)$

Fermentation xylose to product ( $w t \%$ )

CEH xylan to xylose (wt\%)

Fermentation contamination loss (wt\%)

CEH Solids loading ( $w t \%)$

Fermentation arabinose to product (wt\%)

DMR lignin to liquor (wt\%)

Condensation single pass conversion

$$
\begin{array}{r}
(0.85: 1:-) \\
(-: 0: 0.1) \\
(0.85: 0.95: 1) \\
(0.05: 0.1: 0.25) \\
(0.9: 0.963: 0.99) \\
(0.75: 0.85: 0.9) \\
(0.9: 0.989: 0.99) \\
(0.06: 0.03: 0) \\
(0.05: 0.075: 0.1) \\
(0.75: 0.85: 0.9) \\
(0.25: 0.47: 0.6) \\
(0.4: 0.6: 0.9)
\end{array}
$$

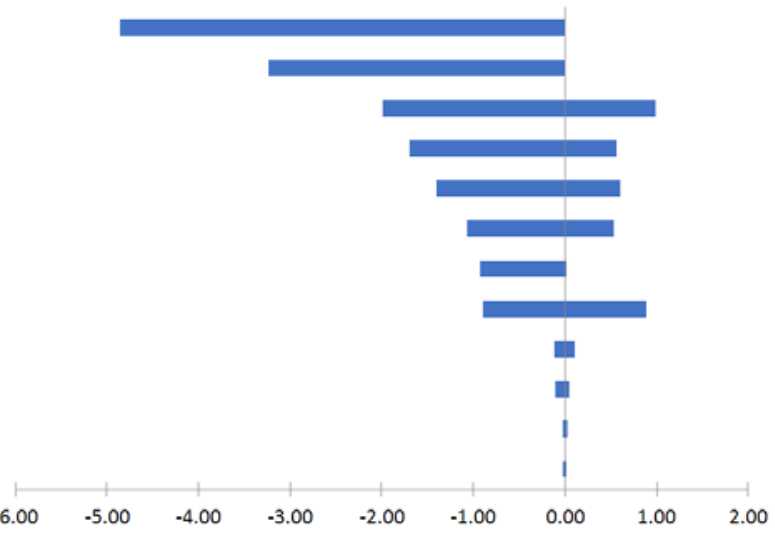

$\Delta$ Yield (MMGGE/yr), Base Case $=\mathbf{3 2 . 5}$

Figure 23. Acids single-point sensitivity tornado charts for MFSP and production yield 


\subsubsection{Single-Point Sensitivity Analysis: BDO to Fuels Pathway}

As in the acids pathway, similar parameters for pretreatment, hydrolysis, fermentation, and upgrading, as well as lignin utilization, were explored for the BDO pathway. Table 43 lists the studied variables, their baseline values, and the associated minima/maxima. The sensitivities of (a) MFSP and (b) fuel product yield are displayed as tornado charts in Figure 24.

Similar to the acids pathway, the uncertainty in capital cost has the largest impact on MFSP and is more pronounced compared to previous designs due to the increase in overall capital associated with an additional lignin processing train, as well as a more complicated catalytic upgrading train. Beyond capital costs, the amount of lignin accessible to the muconate fermentation and the muconate fermentation productivity are again the next largest MFSP drivers. Also similar to the above acids case, for a more near-term interim goal of $\$ 3 /$ GGE by 2022, overall biomass lignin conversion to adipic acid could be relaxed by roughly 12\%, from 266 to $235 \mathrm{lb} /$ dry ton biomass (after also increasing the biomass feedstock cost to the 2022 interim target of $\$ 79.07 /$ dry ton as noted above) at a resulting MFSP of $\$ 2.99 /$ GGE.

For the fuel train via BDO, the largest cost drivers are enzyme loading, DMR caustic loading, and enzymatic hydrolysis solids content (primarily impacting the downstream BDO concentration and thus catalytic upgrading costs). The amount of cellulose hydrolyzed to glucose reflects the largest effect on fuel yield followed by the fermentation conversion of hydrolyzed glucose to product. Similarly but with a smaller magnitude, the net conversion of the minor sugars to products during hydrolysis and fermentation also has a substantial effect on overall fuel yield, which includes xylose and arabinose contributions, but is also affected by the amount of contamination to side product species. As above, the distinction between uncertainty in capital cost estimates and economic parameters compared to process/technical risks should be noted. 
Table 43. Assumptions Varied in the BDO Sensitivity Analysis

\begin{tabular}{|c|c|c|c|c|}
\hline & Assumption & Min MFSP & Baseline & Max MFSP \\
\hline \multirow[t]{3}{*}{ Pretreatment } & DMR $\mathrm{NaOH}$ loading $(\mathrm{mg} / \mathrm{g})$ & 50 & 70 & 100 \\
\hline & DMR xylan to liquor (wt\%) & $25 \%$ & $10 \%$ & $5 \%$ \\
\hline & DMR lignin to liquor (wt\%) & $60 \%$ & $47 \%$ & $25 \%$ \\
\hline \multirow{6}{*}{$\begin{array}{l}\text { Continuous } \\
\text { Enzymatic } \\
\text { Hydrolysis }\end{array}$} & EH Solids loading (wt\%) & $30 \%$ & $25 \%$ & $20 \%$ \\
\hline & EH cellulose to glucose ( $w t \%)$ & $95 \%$ & $90 \%$ & $75 \%$ \\
\hline & EH xylan to xylose (wt\%) & $95 \%$ & $90 \%$ & $75 \%$ \\
\hline & $\mathrm{EH}$ enzyme loading $\mathrm{mg} / \mathrm{g}$ & 5 & 10 & 20 \\
\hline & EH time $(d)$ & 3.5 & 5 & 6 \\
\hline & Flocculant Requirement (g/kg solid) & - & 0 & 20 \\
\hline \multirow{15}{*}{$\begin{array}{l}\text { Enzyme } \\
\text { Production } \\
\text { BDO Fermentation } \\
\text { and Catalysis }\end{array}$} & Enzyme production capital cost & $-50 \%$ & - & $+50 \%$ \\
\hline & Enzyme Licensing & - & 0 & 0.1 \\
\hline & Fermentation contamination loss (wt\%) & $0 \%$ & $3 \%$ & $6 \%$ \\
\hline & Fermentation glucose to product (wt \%) & $100 \%$ & $95 \%$ & $85 \%$ \\
\hline & Fermentation xylose to product (wt\%) & $95 \%$ & $90 \%$ & $80 \%$ \\
\hline & Fermentation arabinose to product (wt \%) & $95 \%$ & $85 \%$ & $75 \%$ \\
\hline & Lignin press S/L capital cost & $-50 \%$ & - & $50 \%$ \\
\hline & Microfilter retentate loss & 0 & 0.01 & 0.03 \\
\hline & BDO upgrading WHSV & 3 & 2 & 1 \\
\hline & Oligomerization reactor WHSV & 3 & 1 & 0.5 \\
\hline & BDO catalysis capital cost & $-50 \%$ & - & $100 \%$ \\
\hline & $\mathrm{H} 2$ price $(\$ / \mathrm{kg})$ & 1 & 1.57 & 2 \\
\hline & BDO upgrading reaction temperature $\left({ }^{\circ} \mathrm{C}\right)$ & 150 & 250 & - \\
\hline & BDO upgrading catalyst cost & $-50 \%$ & - & $50 \%$ \\
\hline & Oligomerization catalyst cost & $-50 \%$ & - & $50 \%$ \\
\hline \multirow[t]{7}{*}{ Lignin Utilization } & Muconic metabolic yield (g/g glu) & 0.408 & 0.3076 & - \\
\hline & Muconic productivity (g/L/hr) & 2 & 1 & 0.5 \\
\hline & Muconic acid fermentation $\mathrm{pH}$ control & none & controlled & - \\
\hline & Muconic acid hydrogenation Temperature $\left({ }^{\circ} \mathrm{C}\right)$ & 100 & 78 & - \\
\hline & Sugar diversion to coproduct ( $w t \%$ clean sugar) & 0.1 & 0 & - \\
\hline & Metabolically accessible lignin (wt\%) & $68 \%$ & $53 \%$ & $41 \%$ \\
\hline & Oslo Crystallizer capital cost & $-20 \%$ & - & $20 \%$ \\
\hline \multirow[t]{2}{*}{ Economics } & Total capital investment $(\mathrm{TCl})$ & $25 \%$ & - & $-25 \%$ \\
\hline & Feedstock Costs (\$/dry ton) & 80 & 71.26 & 60 \\
\hline
\end{tabular}


Total capital investment (TCI)

Metabolically accessible lignin (wt\%)

Muconic productivity $(\mathrm{g} / \mathrm{L} / \mathrm{hr})$

EH enzyme loading $\mathrm{mg} / \mathrm{g}$

DMR NaOH loading $(\mathrm{mg} / \mathrm{g})$

EH Solids loading (wt\%)

Feedstock Costs (\$/dry ton)

BDO upgrading reaction temperature $\left({ }^{\circ} \mathrm{C}\right)$

Flocculant Requirement ( $\mathrm{g} / \mathrm{kg}$ solid)

Fermentation glucose to product (wt\%)

Muconic acid hydrogenation Temperature $\left({ }^{\circ} \mathrm{C}\right)$

DMR xylan to liquor (wt\%)

Muconic metabolic yield (g/g glu)

$\mathrm{H} 2$ price $(\$ / \mathrm{kg})$

Fermentation xylose to product (wt\%)

DMR lignin to liquor (wt\%)

Fermentation contamination loss (wt\%)

BDO catalysis capital cost

Muconic acid fermentation $\mathrm{pH}$ control

Sugar diversion to coproduct (wt\% clean sugar)

EH cellulose to glucose (wt\%)

Microfilter retentate loss

Enzyme production capital cost

EH xylan to xylose (wt\%)

Oligomerization reactor WHSV

Enzyme Licensing

EH time (d)

Oslo Crystallizer capital cost

BDO upgrading WHSV

Lignin press $\mathrm{S} / \mathrm{L}$ capital cost

Oligomerization catalyst cost

Fermentation arabinose to product (wt\%)

$\mathrm{BDO}$ upgrading catalyst cost

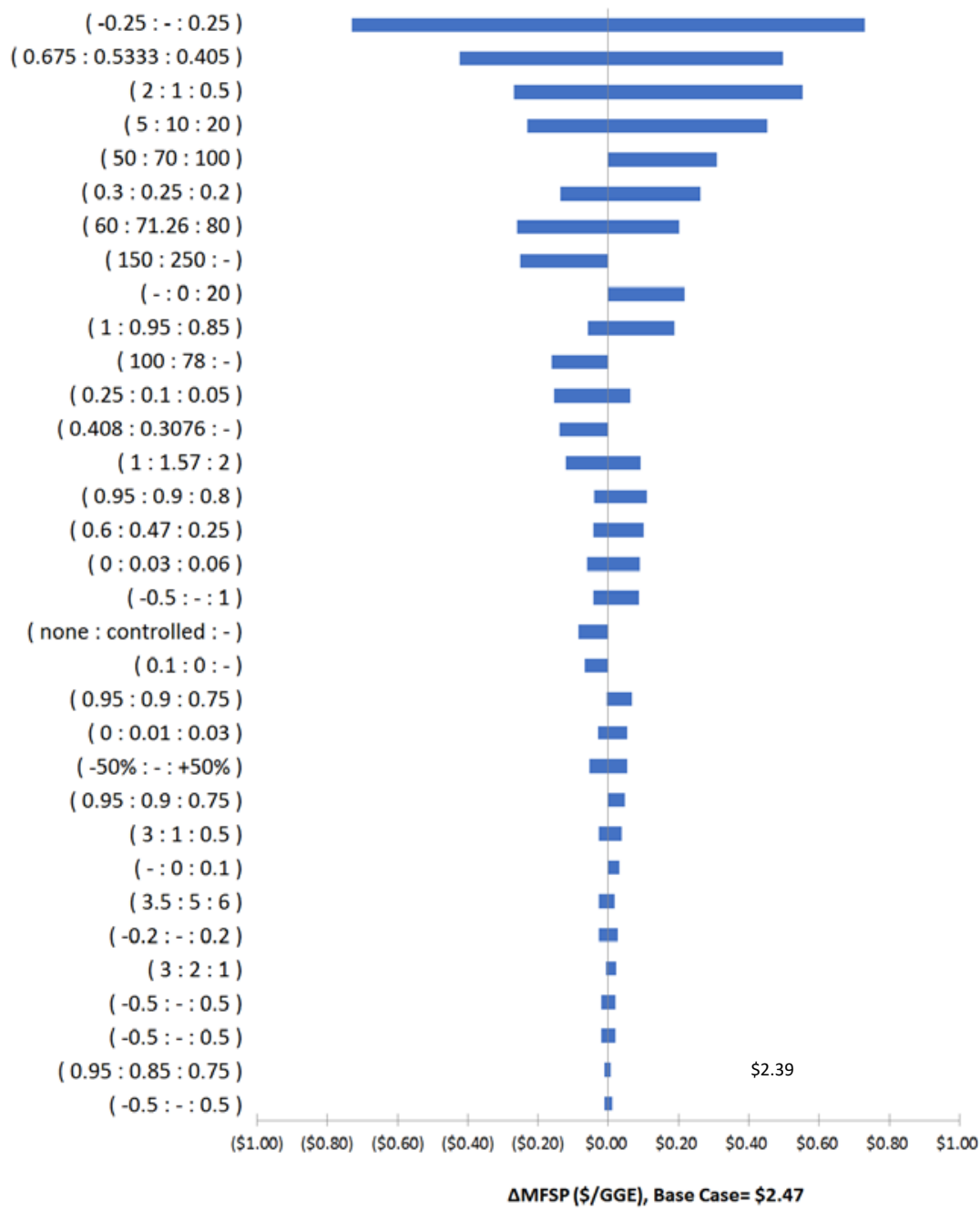

$(0.75: 0.9: 0.95)$

$(-: 0: 0.1)$

( $0.85: 0.95: 1$ )

( $0.75: 0.9: 0.95$ )

( $0.05: 0.1: 0.25$ )

$(0.8: 0.9: 0.95)$

( $0.06: 0.03: 0)$

( $0.03: 0.01: 0$ )

( $0.25: 0.47: 0.6$ )

( $0.75: 0.85: 0.95$ )

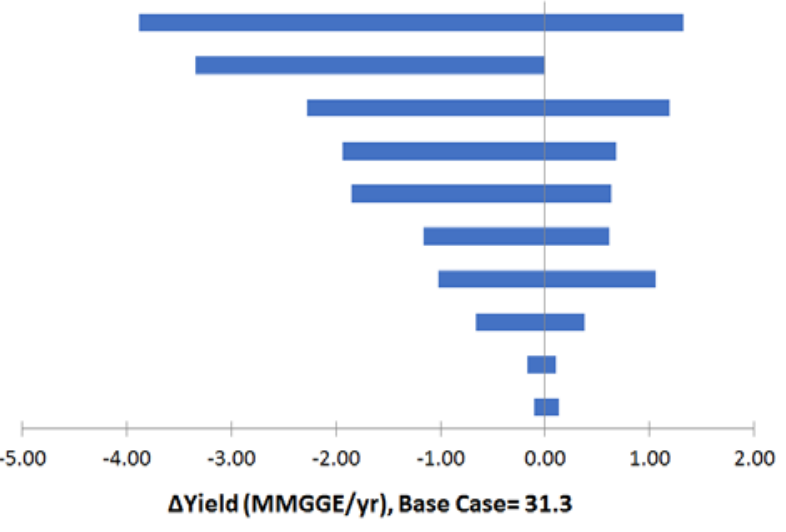

Figure 24. BDO single-point sensitivity tornado charts for MFSP and production yield 


\subsubsection{MFSP Sensitivity to Adipic Acid Co-Product Value}

As noted above, the market value of adipic acid has fluctuated significantly over recent years, as is typical for many chemical products as a function of petroleum prices and market conditions. Given this dynamic, a 15-year average price was selected as the base case here, at $\$ 0.86 / 1 \mathrm{~b}$ (each year's nominal US Gulf Coast price was first inflated to $2016 \$$ and then averaged). To highlight the strong sensitivity this value exhibits on overall biorefinery economics, Figure 25 shows the MFSP implications for adipic acid market value ranging from $\$ 0.5 / \mathrm{lb}$ (representing the lowest price point over that timeframe) up to $\$ 1.25 / \mathrm{lb}$ (representing the point when MFSP would approach $\$ 0 / \mathrm{GGE}$, although the value has exceeded this range). Over that price range, the resulting MFSP for both the acids and BDO pathways varies substantially over a span of $\$ 4.5 / \mathrm{GGE}$, demonstrating that this parameter exhibits a very strong influence on overall biorefinery economics at the base case lignin conversion targets projected here. While this is not a new finding and is in line with prior TEA modeling for similar biorefinery concepts employing a high-value coproduct [157], it highlights the importance of understanding and anticipating market dynamics when planning to construct and operate a biorefinery of this nature, ideally exhibiting the capacity to respond to changing market conditions by producing different products or maximizing fuel versus product outputs. At the base case adipic acid yields projected in this design, the current global market for adipic acid (2.8 MM tonne/year) would be met with approximately 33 biorefineries of this size. However, as noted throughout this report, adipic acid is intended to represent one example of many other potential products from lignin (or sugars), and in reality different biorefineries would be expected to produce different products as prevailing market conditions dictate, just as petrochemical facilities do not all produce the same individual products.

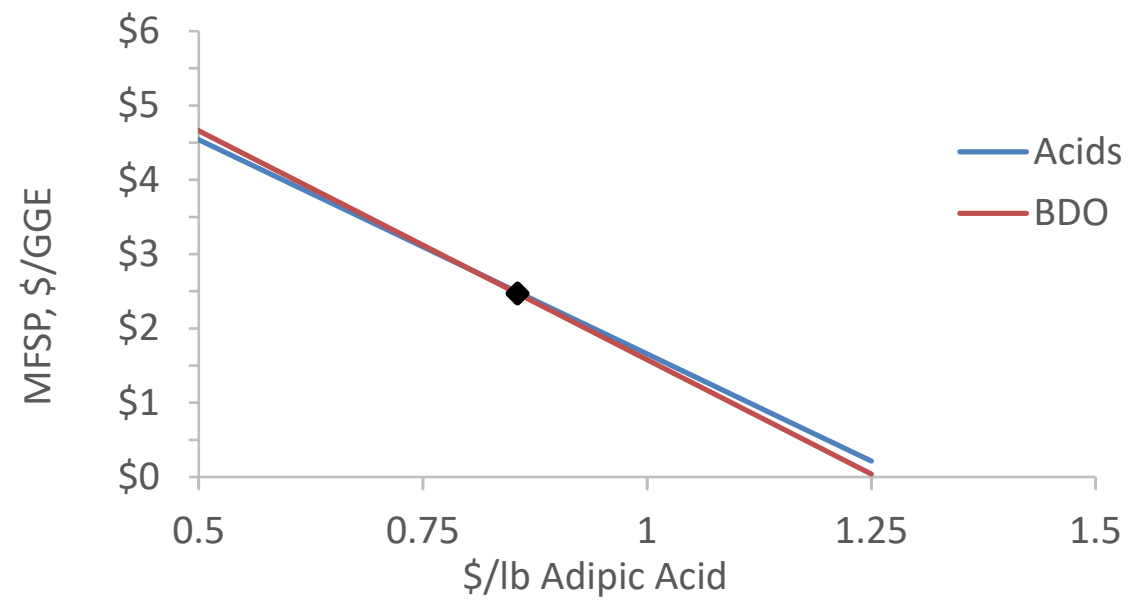

Figure 25. MFSP sensitivity to adipic acid selling price for both fuel train pathways. Marker indicates base case price assumed in this design ( $\$ 0.86 / \mathrm{lb})$ based on 15 -year adipic acid price history. 


\subsection{Sustainability Metric Indicators}

This section presents primary sustainability metric indicators of the current conceptual process at the conversion stage. Direct $\mathrm{CO}_{2}, \mathrm{NO}_{2}$, and $\mathrm{SO}_{2}$ emissions from the biorefinery, water consumption, and other process-related metrics were taken directly from the Aspen conversion process models described above. The material and energy flows of the conversion step capture the impacts of input raw materials, and outputs such as fuel yields, wastes, and coproducts as predicted by the process model, and are shown in Table 44.

The input/output inventories in Table 44 also provide the necessary information required for performing life cycle analysis (LCA) modeling to quantify greenhouse gas (GHG) emissions and fossil energy consumption. The biorefinery GHGs and fossil energy consumption will be calculated separately under supply chain sustainability analysis efforts coordinated by partners at Argonne National Laboratory in the future and are not reported here. A complete well-to-wheel or supply chain LCA evaluation is required to more fully understand the sustainability implications for the full supply chain based on this technology pathway, such as how the overall integrated biorefinery GHG emissions profiles compare to BETO goals relative to petroleum diesel. One key driver in the system LCA is the inclusion of a non-fuel coproduct (e.g., adipic acid and sodium sulfate). When produced in significant quantities, as is the case here, it can strongly influence the overall GHG emissions (with the potential for substantial GHG credits relative to energy-intensive synthesis of a material such as fossilderived adipic acid), depending on the coproduct handling method selected for the LCA [158].

Table 45 summarizes the key sustainability metric indicators for the two conversion processes evaluated here. The fuel yield, carbon efficiency to fuels and chemical coproduct (e.g., adipic acid), as well as the electricity import are comparable for the two cases. The overall combined carbon efficiency (to fuel and adipic acid) for the acids pathway and BDO pathway are roughly $41 \%$ and $40 \%$, respectively. The BDO pathway is more energy-intensive, particularly with respect to aqueous catalytic upgrading, and thus requires natural gas supplementation in the boiler (15.4 MJ/GGE). The acids pathway does not require natural gas supplementation in the boiler, but does use a separate natural gas-fired heater system for hot oil circulation in servicing high-temperature heating needs discussed previously (9.6 MJ/GGE). The acids pathway exhibits higher water consumption at the conversion stage, as discussed previously. On an energy basis, the water consumption for the acid and BDO pathways are $13.5 \mathrm{gal} / \mathrm{GGE}\left(5,041 \mathrm{~m}^{3} /\right.$ day $)$ and $8.9 \mathrm{gal} / \mathrm{GGE}\left(3,203 \mathrm{~m}^{3} /\right.$ day $)$, respectively. Biorefinery net water consumption includes, but is not limited to, water that is incorporated into the product and evaporation directly from process operations or indirectly from cooling and heating processes (e.g., cooling tower evaporative losses). The relatively high water demands for these processes may impose constraints on facility siting locations, requiring sufficient access to local water resources while also being located in areas suitable for meeting delivered feedstock compositional/cost targets discussed in Section 3.1. 
Table 44. Input and Output Inventory Data Associated with the Modeled Conversion Facility

\begin{tabular}{|c|c|c|c|c|}
\hline \multirow{3}{*}{$\begin{array}{l}\text { Pathways (via intermediates) } \\
\text { Products }\end{array}$} & \multicolumn{2}{|l|}{ Acids } & \multicolumn{2}{|l|}{ BDO } \\
\hline & \multicolumn{2}{|c|}{ Production Rate } & \multicolumn{2}{|c|}{ Production Rate } \\
\hline & 11,465 & $\mathrm{~kg} / \mathrm{hr}$ & 11,032 & $\mathrm{~kg} / \mathrm{hr}$ \\
\hline Hydrocarbon Fuel & 504,671 & MJ/hr (LHV) & 486,293 & $\mathrm{MJ} / \mathrm{hr}(\mathrm{LHV})$ \\
\hline \multicolumn{5}{|l|}{ Co-products } \\
\hline Adipic Acid (Polymer Grade) & 10,770 & $\mathrm{~kg} / \mathrm{hr}$ & 11,092 & $\mathrm{~kg} / \mathrm{hr}$ \\
\hline Recovered sodium sulfate salt from WWT & 13,871 & $\mathrm{~kg} / \mathrm{hr}$ & 14,163 & $\mathrm{~kg} / \mathrm{hr}$ \\
\hline Export Electricity & 0 & $\mathrm{~kW}$ & 0 & $\mathrm{~kW}$ \\
\hline Resource Consumption & \multicolumn{2}{|c|}{ Flow Rate (kg/hr) } & \multicolumn{2}{|c|}{ Flow Rate (kg/hr) } \\
\hline Biomass Feedstock (20\% moisture) & \multicolumn{2}{|c|}{104,167} & \multicolumn{2}{|c|}{104,167} \\
\hline Sulfuric Acid, 93\% & \multicolumn{2}{|l|}{10,531} & \multicolumn{2}{|l|}{10,835} \\
\hline Caustic (as pure) & \multicolumn{2}{|l|}{8,235} & \multicolumn{2}{|l|}{8,494} \\
\hline Ammonia & \multicolumn{2}{|l|}{1,359} & \multicolumn{2}{|l|}{1,238} \\
\hline Glucose & \multicolumn{2}{|l|}{1,324} & \multicolumn{2}{|l|}{1,324} \\
\hline Corn Steep Liquor & \multicolumn{2}{|l|}{1,478} & \multicolumn{2}{|l|}{800} \\
\hline Corn oil & \multicolumn{2}{|l|}{7.3} & \multicolumn{2}{|l|}{7.3} \\
\hline Host nutrients & \multicolumn{2}{|l|}{37} & \multicolumn{2}{|l|}{37} \\
\hline Sulfur Dioxide & \multicolumn{2}{|l|}{9.0} & 9.0 & \\
\hline Diammonium Phosphate & 714 & & 627 & \\
\hline Toluene Solvent Makeup & 90 & & 0 & \\
\hline Hydrogen & 0 & & 816 & \\
\hline Ethanol & 37 & & 37 & \\
\hline Boiler Chemicals & 0.2 & & 0.2 & \\
\hline FGD Lime & 97 & & 103 & \\
\hline Cooling Tower Chemicals & 3.4 & & 2.1 & \\
\hline Makeup Water & 209,901 & & 133,396 & \\
\hline Natural Gas for Boiler & 0 & & 1,300 & \\
\hline Natural Gas for Hot Oil System & 37.3 & MMBtu/hr & 0 & \\
\hline Grid Electricity (Net Import) & 44,011 & $\mathrm{~kW}$ & 41,546 & $\mathrm{~kW}$ \\
\hline Waste Streams & Flow Rat & e (kg/hr) & Flow Rate & (kg/hr) \\
\hline Disposal of Ash & 4,270 & & 4,279 & \\
\hline Air Emissions & Flow Rat & e (kg/hr) & Flow Rate & (kg/hr) \\
\hline $\mathrm{H} 2 \mathrm{O}$ & 55,465 & & 62,998 & \\
\hline N2 & 362108 & & 394980 & \\
\hline CO2 (biogenic) & 82,513 & & 83,456 & \\
\hline $\mathrm{CO} 2$ (fossil) & 2,225 & & 3,575 & \\
\hline $\mathrm{O} 2$ & 59,291 & & 57,766 & \\
\hline NO2 & 8.4 & & 19.6 & \\
\hline $\mathrm{SO} 2$ & 5.8 & & 6.7 & \\
\hline $\mathrm{CO}$ & 42.2 & & 53.9 & \\
\hline $\mathrm{CH} 4$ & 0 & & 0 & \\
\hline
\end{tabular}


Table 45. Summary of Sustainability Metric Indicators for the Modeled Biorefinery Process

\begin{tabular}{|l|l|c|c|}
\cline { 3 - 4 } \multicolumn{2}{l|}{} & \multicolumn{2}{c|}{ Pathways (via intermediates) } \\
\hline Sustainability Metrics & Acids & BDO \\
\hline Fuel Yield by Weight of Biomass & GGE per dry ton biomass & 44.8 & 43.2 \\
\hline Carbon Efficiency to Fuels & $\%$ C in feedstock & 26.2 & 25.0 \\
\hline Carbon Efficiency to Adipic Acid & $\%$ C in feedstock & 14.4 & 14.8 \\
\hline Electricity Import & KWh/GGE & 10.7 & 10.5 \\
\hline Natural Gas Import & MJ/GGE & 9.6 & 15.4 \\
\hline Water Consumption & gal/GGE & 13.5 & 8.9 \\
\hline Water Consumption & $\mathrm{m}^{3} / \mathrm{day}$ & 5,041 & 3,203 \\
\hline
\end{tabular}

\subsection{Additional Opportunities for Cost Reduction}

Beyond the process configurations considered here through rigorous TEA modeling, a number of additional opportunities exist to further reduce fuel costs and/or achieve similar cost targets through different approaches. While outside the scope of the present modeling work, these opportunities are briefly discussed on a high level below, and may be evaluated in more detail in the future.

\section{Waste $\mathrm{CO}_{2}$ /Biogas Utilization}

In this design, vented gas from sugar fermentation, lignin conversion, and boiler flue gas represent a substantial amount of wasted carbon as $\mathrm{CO}_{2}$ (roughly $26,000 \mathrm{~kg} / \mathrm{hr}, 10,000 \mathrm{~kg} / \mathrm{hr}$, and $78,000 \mathrm{~kg} / \mathrm{hr}$, respectively). The flue gas from the boiler is the main $\mathrm{CO}_{2}$ stream taking up $\sim 60 \%$ of the total $\mathrm{CO}_{2}$ produced in the process. Conversion of waste $\mathrm{CO}_{2}$ into transportation fuels and chemicals represents a significant opportunity for transforming abundant gaseous resources into a variety of materials and chemicals. However, even with this large potential, commercial expansion in this area has been limited due to the lack of information and understanding around the economic viability of the emerging $\mathrm{CO}_{2}$ conversion technologies.

Highlighting only reductive pathways, four pathways stand out for $\mathrm{CO}_{2}$ upgrading to chemicals and fuels: biochemical, thermochemical, electrochemical, and bioelectrochemical. A microbial-assisted $\mathrm{CO}_{2}$ electrolyer is a promising option to integrate wastewater treatment and waste $\mathrm{CO}_{2}$ utilization. Bioelectrochemical systems are shown to be more effective at treating wastewater that contains high concentrations of volatile fatty acids, such as wastewater produced by hydrolysis and fermentation. In the present design, the bioelectrolysis process has the potential to replace the aerobic digestion step, which followed anaerobic digestion in previous biochemical design models for furture wastewater treatment. Depending on the configuration and routing of DMR liquor, acetate salts may reach wastewater treatment, which are very suitable for bioelectrochemcial systems.

$\mathrm{CO}_{2}$ can be used as a substrate to generate methane $\left(\mathrm{CH}_{4}\right)$ by using enriched methanogens or can be converted to metabolites with $\mathrm{CH}_{4}$. Recently, there has been increasing interest in co-utilization of one-carbon compounds such as $\mathrm{CH}_{4}$ or $\mathrm{CO}_{2}$ as substrates, not only because of their abundance, but also because both are produced from conventional anaerobic digestion of waste materials. Moving forward, opportunities to perform comparative analyses would allow studying a broad range of technologies converting $\mathrm{CO}_{2}$ and $\mathrm{CH}_{4}$ to fuels and chemicals to understand their potential economic and environmental benefits on previous or ongoing biomass conversion pathways funded by BETO. Specifically, process feasibility and TEA modeling for waste gaseous carbon utilization within integrated biorefinery systems such as this would allow for understanding technical performance 
levels that would be required to offset the added costs for such operations in order to realize net economic benefits to the biorefinery. Gaining better understanding of new reaction mechanisms and opportunities to optimize carbon flux/energy efficiency may allow for highlighting paths to improved economics and relaxing targets for other challenging parameters elsewhere in the integrated process.

\section{Methane to Products}

Similar to the above concept, microbial conversion of biogas using natural $\mathrm{CH}_{4}$-consuming bacteria (methanotrophs) offers broad and highly selective valorization potential. Methanotrophic bacteria are characterized by their ability to utilize a variety of different $\mathrm{C} 1$ substrates, including biogas generated from wastewater treatment facilities, as sole carbon and energy sources. Previous research using methanotrophic bacteria has demonstrated production of lactic acid, muconic acid, succinic acid, acetic acid, formic acid, and lipids [159-161]. NREL researchers have explored numerous methane to chemical pathways through process and market analyses and recommended several chemical intermediates for future R\&D, including microbial biomass, sucrose, butanediol, succinic acid, and additional hydrocarbon fuel intermediates (NREL unpublished results). A series of process configurations are planned to be investigated for insertion of biogas fermentation into current process designs, with gas fermentation products serving as inputs at different locations, including (a) biomass pretreatment, (b) biological conversion, (c) product upgrading, or (d) coproduct production to increase overall biorefinery carbon efficiency.

Although the present design configuration has eliminated the anaerobic digestion unit from the WWT system (and thus the primary source of biogas), this is primarily a reflection of the aggressive assertions made around the lignin/residuals upgrading train (Area 700) and the high targeted conversions of all components that were previously relegated to WWT. In the event such high conversions could not be achieved or a different configuration strategy was pursued (as outlined below), the COD loading to the WWT system would increase again, which could require reintroducing the anaerobic digestion (AD) unit. Thus, as a "risk mitigation strategy" it will be useful moving forward to evaluate AD biogas utilization opportunities for increased carbon retention and improved economics. One such option is sucrose production from biogas, utilizing bubble columns to capture and convert a portion of the biogas methane. The sucrose could subsequently be converted to fuel precursors at high utilization efficiencies through either the BDO or acid pathways or could alternatively be used in the lignin upgrading train to produce additional muconic acid coproduct. Alternatively, a more economical option may be to produce either the fuel or coproduct molecules directly in the biogas upgrading bioreactor without the sucrose intermediate, if sufficiently high yields and productivities could be achieved. Another option may also be directly selling the produced sucrose as a coproduct (although this would be relatively low-value being tied to sucrose market prices) or producing single-cell protein for animal feed markets.

\section{Alternative Uses for Lignin/Carbohydrates}

A key driver for meeting the out-year $\$ 2.50 / \mathrm{GGE}$ cost goal is maximizing the utilization of all biomass constituents in a highly efficient and integrated conversion strategy. Given the importance of maximizing the value of biomass and valorizing all components, particularly lignin, further improvements to conversion yield and efficiency may be realized by utilizing alternative strategies for biomass deconstruction with one out-year option focused on a "lignin first" deconstruction method. While this concept is in an early stage of development, the ultimate objective of such an option is to maximize the removal and conversion of lignin while minimizing losses of carbohydrates. Removing 
the recalcitrant portion of the biomass, such as lignin, has been shown to reduce pretreatment severity and reduce enzyme loading for sugar production. The deconstruction strategy chosen, however, must be tailored for downstream conversion. Chemical catalytic processes that integrate deconstruction of residual solids with catalytic upgrading, i.e. the RCF concepts discussed above, are targeting highvalue chemicals and fuels from lignin [162-164]. Recent efforts have focused on improving both carbon selectivity and atom efficient conversion for these upgrading options, as well as driving down catalyst costs.

Alternatively, more traditional thermochemical routes such as pyrolysis also offer an additional opportunity to upgrade residual lignin to fuel blendstocks. These strategies for lignin conversion to fuels could also open up the opportunity to instead utilize sugars for the production of high-value chemical coproducts and further integrate with pathways being pursued under the DOE BETOsupported Agile BioFoundry project, which aims to advance early-stage metabolic science to accelerate the rate of strain improvements and to develop robust, scalable organisms for fuel and product manufacturing [165]. By focusing on a diverse spectrum of product options from both sugars and lignin across a range of upgrading strategies, these efforts have aimed to reduce the risk of saturating chemicals markets and depressing chemical prices while boosting the value of traditionally underutilized fractions of the biomass. Given the projected growth of the bio-industry and the number of biorefineries needed to meet out-year fuel production needs, a range of coproduct strategies will be required to help enable low-cost biofuels.

Finally, a more simplistic route to reduce MFSPs while maintaining the overall structure of the process configurations would be to divert a fraction of the sugar fermentation intermediates away to coproducts. To minimize costs and avoid introducing further process complexity through a third processing train to a completely different product, the most practical approach here would be to isolate products already being produced or which could easily be produced from the existing upgrading steps as value-added coproducts. In the case of BDO, logical choices could be 1,3-butadiene or MEK, both produced through dehydration reactions from 2,3-BDO (there is not a large market for the 2,3-BDO component itself, although there is a considerable market for 1,4-BDO). In the case of the acids pathway, isolating ketone intermediates (i.e., 4-heptanone) may present opportunities for sale into solvent markets or related products. 


\section{Concluding Remarks}

\subsection{Summary}

This report establishes a plausible case for achieving an ultimate cost goal below $\$ 2.50 / \mathrm{GGE}$ of upgraded renewable hydrocarbon fuels by 2030 via biological production, based on two conceptual integrated processing pathways to hydrocarbon fuels coupled with bio-derived coproducts. Namely, the models discussed here assume continued improvements are made in the biomass deconstruction areas, including more novel approaches to alkaline extraction and mechanical refining pretreatment, as well as (in one case) continuous enzymatic hydrolysis, beyond performance demonstrated in prior state of technology benchmarking efforts. This includes demonstrating high solubilization of lignin, extractives, acetate, ash, and other non-fermentable components while minimizing losses of fermentable components and maximizing concentration of DMR black liquor through the use of a counter-current alkaline extraction operation. For the case of continuous enzymatic hydrolysis, this also includes demonstrating nearly $100 \%$ conversions of carbohydrates to monomeric sugars at a combined enzyme loading of $10 \mathrm{mg} / \mathrm{g}$ cellulose. These front-end unit operations leverage NREL expertise and prior work in these areas and will remain important areas of future research for continued improvement.

In addition to biomass deconstruction to sugars, the design also sets targets for sugar upgrading to fuels and importantly, lignin and other residual component upgrading to coproducts. On the former, two pathways to bioconversion intermediates are considered - carboxylic acids and 2,3-BDO, both under high sugar conversion efficiencies (95\% glucose, $85 \%-90 \%$ xylose, and $85 \%$ arabinose to products) and intermediate product yields ( $>88 \%$ of theoretical). These intermediates are subsequently routed through catalytic upgrading reactions to expand chain lengths to fuel-range components as well as deoxygenate/hydrogenate the catalytic intermediates to finished hydrocarbon products, with high targeted yield and selectivity to desired fuel products. In light of the high demands and resultant costs for caustic and acid inputs throughout the process (contributing roughly $\$ 1.30 / \mathrm{GGE}$ to overall MFSPs), it will be critical to either recover and recycle the majority of these chemicals through advanced separation technologies or to offset a fraction of those costs through the sale of resultant sodium sulfate salt isolated downstream, with this model reflecting the latter approach (at an average coproduct savings of roughly $\$ 0.54 / \mathrm{GGE}$, offsetting approximately $40 \%$ of the raw chemical costs in either pathway).

The majority of all biological and catalytic upgrading steps for both fuel train scenarios have largely been demonstrated at performance levels that support meeting final targets by 2030 and in many cases sooner, reflective of paths to first achieve $\$ 3 / \mathrm{GGE}$ interim goals by 2022 prior to final goals below $\$ 2.50 /$ GGE by 2030 . However, the targets set forward for the lignin-to-coproduct train represent newer research and may be reflective of a longer timescale closer to the final 2030 out-years, particularly with respect to lignin deconstruction to convertible monomers, targeted at $53 \%$ of the original biomass lignin (inclusive of both deconstruction across DMR pretreatment as well as subsequently across $\mathrm{BCD}$ in the lignin train). After that point, lignin train bioconversion and product recovery/upgrading are generally more straightforward based on performance observed to date, with targets set at $1 \mathrm{~g} / \mathrm{L}-\mathrm{hr}$ muconic acid productivity, $0.315 \mathrm{~g} / \mathrm{g}$ muconic acid yield from lignin monomers, $0.93 \mathrm{~g} / \mathrm{g}$ muconic acid yield from other residual components (extractives and carbohydrates), and $95 \%$ net recovery and conversion of muconic to adipic acid (inclusive of muconic and adipic acid purification as well as muconic acid hydrogenation). 
The end result of the techno-economic analysis was a predicted MFSP of $\$ 2.47 / \mathrm{GGE}$ for the BDO pathway and $\$ 2.49 /$ GGE for the acids pathway (2016\$) at a final upgraded fuel product yield of 43.2 and $44.8 \mathrm{GGE} /$ dry ton of biomass for the two respective pathways. This reflects a $\$ 1.65 / \mathrm{GGE}$ and $\$ 1.59 /$ GGE contribution from feedstock at $\$ 71.26 /$ dry ton, and a $\$ 3.83 /$ GGE and $\$ 3.87 /$ GGE contribution from the fuel train conversion process for the BDO and acids pathways, respectively. Critical to either pathway's ability to ultimately meet MFSPs below $\$ 2.50 / \mathrm{GGE}$ is the inclusion of coproducts derived from lignin (and other residual components); in this work, adipic acid is reflected as a representative example coproduct with a targeted yield of 266 and $259 \mathrm{lb} /$ dry ton, which translates to substantial coproduct offsets to the MFSP at negative \$3.00/GGE and \$2.97/GGE for the BDO and acids pathways, respectively. At those yields, the current global market for adipic acid (2.8 MM tonne/year) would be met with approximately 33 biorefineries of this size. However, we stress that adipic acid is intended to represent one example of many other potential products from lignin (or sugars), and in reality different biorefineries would be expected to produce different products as prevailing market conditions dictate, just as petrochemical facilities do not all produce the same products. In the more near term, in order to achieve interim MFSP goals of $\$ 3 /$ GGE by 2022 , the majority of the fuel train targets are anticipated to likely still be achievable within that timeframe, which would relax the lignin coproduct assumptions to only require 235 and $229 \mathrm{lb} / \mathrm{dry}$ ton net adipic acid yields for the BDO and acids pathways, respectively (after adjusting feedstock costs to interim 2022 goals of $\$ 79.07 /$ dry ton). This translates primarily to reduced lignin deconstruction to monomers at roughly $43 \%$, if simplistically focused on adjusting the single most challenging metric in isolation.

In addition to providing an economic analysis, the present report also considers key sustainability metric indicators that may be important factors in an overall LCA of the system. For the BDO pathway, these metrics were estimated at $25 \%$ carbon yield from biomass to fuels, $10.5 \mathrm{KWh} / \mathrm{GGE}$ net power import, $15.4 \mathrm{MJ} / \mathrm{GGE}$ natural gas consumption, and $8.9 \mathrm{gal} / \mathrm{GGE}$ net water demands for the biorefinery. For the acids pathway, these were estimated at $26 \%$ carbon yield, $10.7 \mathrm{KWh} / \mathrm{GGE}$ power import, 9.6 MJ/GGE natural gas consumption, and $13.5 \mathrm{gal} / \mathrm{GGE}$ net water demand. The coproduction of bio-derived adipic acid may provide substantial credits to overall biorefinery greenhouse gas emission profiles when compared to more energy-intensive fossil-derived synthesis for this product (dependent on LCA coproduct handling methods), but this is outside the scope of the present analysis.

The modeled selling prices are strictly representative of $n^{\text {th }}$-plant assumptions regarding biorefinery design, operation, and financing, and are not intended to reflect first-of-a-kind or early-entry commercial facilities. While the fuel yields presented here are lower and the costs to produce those fuels are higher than what may be possible for cellulosic ethanol, the technology pathways described in this report are more representative of a true "biorefinery" that converts biomass to fungible hydrocarbon fuel products (which may be directly utilized by the existing fuel infrastructure), as well as advantaged bioproducts with relatively straightforward synthesis routes that leverage the compositional characteristics of the biomass feedstock. Within the context of such biorefinery concepts, additional alternative process integration strategies are also possible, including conversion of waste gaseous carbon for increased biorefinery carbon yields, or a number of different options for alternate processing approaches to convert lignin and/or carbohydrates to fuels or coproducts. These were not considered through rigorous TEA in the present scope but may be evaluated moving forward as additional risk mitigation strategies in meeting the $\$ 2.50 / \mathrm{GGE}$ targets in the future. Given the relatively high capital expenses in this design to produce both hydrocarbon fuels and coproducts as required to achieve the $\$ 2.50 /$ GGE goal (roughly $\$ 700 \mathrm{MM}$ or more TCI for both pathways), in the near-term it may be expected for early commercial pioneer facilities to focus on products alone to 
reduce complexity and cost and improve overall process economics, in transitioning to products and fuels under $n^{\text {th }}$-plant scenarios.

It is worthwhile to reiterate that cellulosic ethanol production via fermentative pathways is supported by decades of research and process understanding, both at NREL and elsewhere, thus carrying a level of certainty and credibility when making target projections for biochemical ethanol TEA modeling, as in the case of the 2011 and even 2002 ethanol design reports. In contrast, biological hydrocarbon production is a much newer and more novel approach to biological conversion of sugars, with doors that have only recently been opened to a number of product pathways given recent advances in metabolic engineering, which continues to be a rapidly evolving field in this context. This point is even more relevant for lignin upgrading to coproducts, which is a challenge that has been studied for many years but has only recently demonstrated more substantial progress. Thus, the absolute cost values established here, as well as the timeframe required to achieve these outcomes, inherently carries a somewhat higher degree of uncertainty given the nascent stage of research as presented in the public domain.

\subsection{Future Work}

Moving forward, to ultimately achieve cost goals as well as reduce uncertainty in key areas for the modeled processes evaluated here, a number of important bottlenecks, uncertainties, and areas for further development are summarized below. A number of these points are similar to those raised in NREL's 2013 biological design case and remain equally pertinent here:

- Investigate synergistic opportunities for biomass deconstruction/sugar production and process integration: Tailoring the hydrolysate stream to the microorganism tolerance and bioreactor operation will be essential to improving yield and lowering production cost, and there continue to be further opportunities for synergistic improvement in combining unit operations or otherwise simplifying the fully integrated process. Moving to continuous enzymatic hydrolysis is one such example being better tailored to the acids pathway, which requires separating solids prior to bioconversion (otherwise incurring high costs for vacuum belt filtration with a flocculant), versus the BDO pathway which may process whole slurry hydrolysate through bioconversion and thus is better suited for standard batch hydrolysis. Additionally, more room exists for optimization and improved understanding of the proposed counter-current alkaline extraction unit currently envisioned in the DMR step.

- Improve understanding of advanced bioreactor designs: Both fuel train pathways have the potential to require more novel bioreactor designs and operating strategies, i.e., pertractive fermentation for the acids pathway and the potential for microaerophilic fermentation for the BDO pathway (in the event the organism cannot be engineered to eliminate oxygen redox balancing needs). Current design and cost estimates for such equipment are not as well established as more standard bioreactors for fully anaerobic or fully aerobic systems that NREL has considered in the past, and may benefit from further refinements moving forward.

- Maximize sugar (and/or carbon) utilization and microbe metabolic performance: Further work remains on strain engineering opportunities for the Z. mobilis BDO organism with respect to improving xylose/arabinose uptake, minimizing side-product formation, and eliminating oxygen demands to be replaced with alternative options for cell redox balancing (such as coproduction of hydrogen or succinic acid). Similarly, the Clostridium acids organism 
also may have further room for improvement on the former two metrics, and more importantly on demonstrating cost-effective fermentation approaches avoiding costly $\mathrm{pH}$ control (e.g., pertractive acids recovery, low-pH tolerant organisms, or $\mathrm{pH}$ control with subsequent salt recovery strategies). Better understanding is needed as to optimal sugar concentrations in both fermentation pathways, and potential effects of hydrolysate inhibitors. Likewise, more work remains with the $P$. putida organism for lignin monomer (and other available components) fermentation with respect to integrating the multiple metabolic strategies into one single strain and reducing the impact of native regulatory responses preventing desired co-consumption behavior. Additionally, further metabolic modeling and fermentation optimization assessing the tradeoffs in yield versus productivity provides a process-oriented opportunity for improvement (e.g., fed-batch, reactor design, nutrient demands, substrate concentrations, etc.).

- Improve catalyst performance for intermediate upgrading: The present pathway models include a number of catalysis steps for upgrading both the fuel and coproduct intermediates. Current research is ongoing, but generally still in a relatively early state of development, around the majority of the catalytic upgrading steps considered here with respect to reactor operating conditions, space velocities, yields, selectivities, and catalyst material costs (efforts that are primarily coordinated under the ChemCatBio Consortium). As further details on such performance metrics and opportunities for future optimization emerge, the models will be refined accordingly, and improvements may be reflected in future state of technology benchmarks. Furthermore, the potential for refinery integration has not been extensively investigated for these biochemically derived intermediate components, and there may be opportunities for further cost reduction in pursuing such integration strategies, i.e., for final hydrotreating steps of the fuel precursor components to be handled centrally in a refinery rather than at a much smaller scale for these modeled facilities.

- Improve lignin deconstruction and upgrading metrics: The primary challenge of the lignin conversion targets set forth here will be effective lignin deconstruction to convertible monomers, thus this metric in particular deserves further attention and research on further optimization possibilities. Additionally, the present model is challenged by the granularity in characterization of the lignin monomer/oligomer properties and composition. While generally, lignin will proceed through the current valorization scheme regardless of specific chemical monomer structure (e.g., vanillin, ferulic acid, p-coumarate, etc.), both the deconstruction to monomers and the subsequent biological upgrading to muconic acid will display variations based on the specific makeup of soluble lignin. For example, during the aerobic upgrading, variations in oxidation state and the number of oxygen atoms on the monomer will play a role in the amount of oxygen demands, which can be a major cost driver.

- Evaluate alternative integration strategies: Alternate integration strategies may also provide new routes to the MFSP targets presented here, which were not evaluated in the present work but may be considered moving forward. These may include alternative approaches for lignin fractionation and/or thermochemical conversion to fuels or products, thus unlocking other options for sugars to instead be routed to products; or alternatively, pursuing utilization of waste gaseous streams for conversion to additional fuel/product opportunities. 


\section{References}

NREL milestone reports cited below cannot be accessed outside of NREL and DOE. Readers may contact the authors of the specific reference to determine if this information has been made public since publication of this design report.

1. Wooley, R.J., M. Ruth, J. Sheehan, K. Ibsen, and H. Majdeski, A. Galvez. 1999.

Lignocellulosic Biomass to Ethanol Process Design and Economics Utilizing Co-Current Dilute Acid Prehydrolysis and Enzymatic Hydrolysis Current and Futuristic Scenarios. NREL/TP-580-26157. Golden, CO: National Renewable Energy Laboratory.

2. Humbird, D., R. Davis, L. Tao, C. Kinchin, D. Hsu, A. Aden, P. Schoen, J. Lukas, B. Olthof, M. Worley, D. Sexton, and D. Dudgeon. 2011. Process Design and Economics for Biochemical Conversion of Lignocellulosic Biomass to Ethanol: Dilute-Acid Pretreatment and Enzymatic Hydrolysis of Corn Stover. NREL/TP-5100-47764. Golden, CO: National Renewable Energy Laboratory.

3. Aden, A., M. Ruth, K. Ibsen, J. Jechura, K. Neeves, J. Sheehan, B. Wallace, L. Montague, A. Slayton, and J. Lukas. 2002. Lignocellulosic Biomass to Ethanol Process Design and Economics Utilizing Co-Current Dilute Acid Prehydrolysis and Enzymatic Hydrolysis for Corn Stover. NREL/TP510-32438. Golden, CO: National Renewable Energy Laboratory.

4. Schell, D. 2013. "Biochemical Processing Integration." Presented at the DOE Bioenergy Technologies Office Project Peer Review, Alexandria, Virginia, May 20-24, 2013.

5. $\quad$ Davis, R. 2013. "Biochemical Platform Analysis." Presented at the DOE Bioenergy Technologies Office Project Peer Review, Alexandria, Virginia, May 20-24, 2013.

6. Tao, L., et al. 2012. "FY 2012 Biochemical Platform State of Technology Report." NREL milestone report.

7. Tao, L., D. Schell, R. Davis, E. Tan, R. Elander, and A. Bratis. 2014. NREL 2012 Achievement of Ethanol Cost Targets: Biochemical Ethanol Fermentation via Dilute-Acid Pretreatment and Enzymatic Hydrolysis of Corn Stover. NREL/TP-5100-61563. Golden, CO: National Renewable Energy Laboratory.

8. U.S. Department of Energy. 2013. Bioenergy Technologies Office Multi-Year Program Plan. Washington, DC: U.S. Department of Energy.

9. Davis, R., L. Tao, C. Scarlata, E.C.D. Tan, J. Ross, J. Lukas, and D. Sexton. 2015. Process Design and Economics for the Conversion of Lignocellulosic Biomass to Hydrocarbons: Dilute-Acid and Enzymatic Deconstruction of Biomass to Sugars and Catalytic Conversion of Sugars to Hydrocarbons. NREL/TP-5100-62498. Golden, CO: National Renewable Energy Laboratory.

10. Davis, R., L. Tao, E.C.D. Tan, M J. Biddy, G.T. Beckham, C. Scarlata, and J. Jacobson, et al. 2013. Process Design and Economics for the Conversion of Lignocellulosic Biomass to Hydrocarbons-Dilute-Acid and Enzymatic Deconstruction of Biomass to Sugars and Biological 
Conversion of Sugars to Hydrocarbons. NREL/TP-5100-60223. Golden, CO: National Renewable Energy Laboratory.

11. Biddy, M.J., C. Scarlata, and C. Kinchin. 2016. Chemicals from Biomass: A Market Assessment of Bioproducts with Near-Term Potential. NREL/TP-5100-65509. Golden, CO: National Renewable Energy Laboratory.

12. Davis, R. 2017. "Biochemical Platform Analysis." Presented at the DOE Bioenergy Technologies Office Project Peer Review, Denver, Colorado, March 7, 2017.

13. Humbird, D., R. Davis, and J. McMillan. 2017. "Aeration costs in stirred-tank and bubble column bioreactors." Biochemical Engineering Journal 127: 161-166. https://doi.org/10.1016/j.bej.2017.08.006.

14. Yang, Shihui, Ali Mohagheghi, Mary Ann Franden, Yat-Chen Chou, Xiaowen Chen, Nancy Dowe, Michael E. Himmel, and Min Zhang. 2016. "Metabolic engineering of Zymomonas mobilis for 2, 3-butanediol production from lignocellulosic biomass sugars." Biotechnology for Biofuels 9, no. 1: 189. https://dx.doi.org/10.1186\%2Fs13068-016-0606-y.

15. AspenPlus (Release 7.2). 2007. Cambridge, MA: Aspen Technology Inc.

16. Christensen, Peter, and Larry R. Dysert. 2016. Cost Estimate Classification System - As Applied in Engineering, Procurement, and Construction for the Process Industries, 18R-97. Morgantown, WV: AACE International.

17. Cran, J. 1981. "Improved factored method gives better preliminary cost estimates." Chemical Engineer 88, no. 7: 65-79.

18. Wooley, R.J., V. Putsche, and K. Ibsen. 1999. Development of an ASPEN PLUS Physical Property Database for Biofuels Components. NREL Technical Memo, Document \#4141. Golden, CO: National Renewable Energy Laboratory: Golden, CO.

19. Wooley, R.J. and V. Putsche. 1996. Development of an ASPEN PLUS Physical Property Database for Biofuels Components. NREL/TP-425-20685. Golden, CO: National Renewable Energy Laboratory: Golden, CO.

20. Hess, J.R. 2010. "Uniform Format Design and Depot Preprocessing." Presented at the Pacific NorthWest Economic Region's 2010 Annual Summit, Calgary, Alberta, July 16-20, 2010.

21. Chen, Shou-Feng, Richard A. Mowery, Christopher J. Scarlata, and C. Kevin Chambliss. 2007. "Compositional analysis of water-soluble materials in corn stover." Journal of Agricultural and Food Chemistry 55, no. 15: 5912-5918. https://dx.doi.org/10.1021/jf0700327

22. U.S. Department of Energy Office of Energy Efficiency and Renewable Energy Fuel Cell Technologies Office. 2012. "Lower and higher heating values of fuels." https://h2tools.org/hyarc/calculator-tools/lower-and-higher-heating-values-fuels. 
23. U.S. Department of Energy. 2016. 2016 Billion-Ton Report: Advancing Domestic Resources for a Thriving Bioeconomy. Langholtz, M., B. Stokes, and L. Eaton, leads. ORNL/TM-2016/160. Oak Ridge, TN: Oak Ridge National Laboratory.

24. Bonner, Ian J., Kara G. Cafferty, David J. Muth, Jr., Mark D. Tomer, David E. James, Sarah A. Porter, and Douglas L. Karlen. 2014. "Opportunities for energy crop production based on subfield scale distribution of profitability." Energies 7, no. 10: 6509-6526.

https://dx.doi.org/10.3390/en7106509.

25. Ssegane, Herbert, M., Cristina Negri, John Quinn, and Meltem Urgun-Demirtas. 2015. "Multifunctional landscapes: Site characterization and field-scale design to incorporate biomass production into an agricultural system." Biomass and Bioenergy 80: 179-190. https://doi.org/10.1016/j.biombioe.2015.04.012.

26. Stoof, Cathelijne R., Brian K. Richards, Peter B. Woodbury, Eric S. Fabio, Alice R. Brumbach, Jerry Cherney, and Srabani Das, et al. 2015. "Untapped potential: opportunities and challenges for sustainable bioenergy production from marginal lands in the Northeast USA." BioEnergy Research 8 , no. 2: 482-501. https://doi.org/10.1007/s12155-014-9515-8.

27. Werling, Ben P., Timothy L. Dickson, Rufus Isaacs, Hannah Gaines, Claudio Gratton, Katherine L. Gross, and Heidi Liere, et al. 2014. "Perennial grasslands enhance biodiversity and multiple ecosystem services in bioenergy landscapes." Proceedings of the National Academy of Sciences 111, no. 4: 1652-1657. https://doi.org/10.1073/pnas.1309492111.

28. Dauber, J., C. Brown, A. L. Fernando, J. Finnan, E. Krasuska, J. Ponitka, and D. Styles, et al. 2012. "Bioenergy from 'surplus' land: environmental and socio-economic implications." BioRiskBiodiversity and Ecosystem Risk Assessment 7: 5-50. https://doi.org/10.3897/biorisk.7.3036.

29. Valentine, John, John Clifton-Brown, Astley Hastings, Paul Robson, Gordon Allison, and Pete Smith. 2012. "Food vs. fuel: the use of land for lignocellulosic 'next generation' energy crops that minimize competition with primary food production." GCB Bioenergy 4, no. 1: 1-19. https://doi.org/10.1111/j.1757-1707.2011.01111.x.

30. Lacey, Jeffrey A., John E. Aston, Tyler L. Westover, Robert S. Cherry, and David N. Thompson. 2015. "Removal of introduced inorganic content from chipped forest residues via air classification." Fuel 160: p. 265-273. https://doi.org/10.1016/j.fuel.2015.07.100.

31. Thompson, Vicki S., Jeffrey A. Lacey, Damon Hartley, Michael A. Jindra, John E. Aston, and David N. Thompson. 2016. "Application of air classification and formulation to manage feedstock cost, quality and availability for bioenergy." Fuel 180: 497-505. https://doi.org/10.1016/j.fuel.2016.04.040.

32. Lacey, Jeffrey A., Rachel M. Emerson, David N. Thompson, and Tyler L. Westover. 2016. "Ash reduction strategies in corn stover facilitated by anatomical and size fractionation." Biomass and Bioenergy 90: 173-180. https://doi.org/10.1016/j.biombioe.2016.04.006. 
33. Yancey, N., C.T. Wright, and T.L. Westover. 2013. "Optimizing hammer mill performance through screen selection and hammer design." Biofuels 4, no. 1: 85-94.

https://doi.org/10.4155/bfs.12.77.

34. Aston, John E., David N. Thompson, and Tyler L. Westover. 2016. "Performance assessment of dilute-acid leaching to improve corn stover quality for thermochemical conversion." Fuel 186: 311319. https://doi.org/10.1016/j.fuel.2016.08.0 56.

35. Tumuluru, J.S. 2014. "Effect of process variables on the density and durability of the pellets made from high moisture corn stover." Biosystems Engineering 119: 44-57. https://doi.org/10.1016/j.biosystemseng.2013.11.012.

36. Tumuluru, J.S. 2015. "High moisture corn stover pelleting in a flat die pellet mill fitted with a $6 \mathrm{~mm}$ die: physical properties and specific energy consumption." Energy Science \& Engineering 3, no. 4: 327-341. https://doi.org/10.1002/ese3.74.

37. Tumuluru, J.S. 2016. "Specific energy consumption and quality of wood pellets produced using high-moisture lodgepole pine grind in a flat die pellet mill." Chemical Engineering Research and Design 110: 82-97. https://doi.org/10.1016/j.cherd.2016.04.007.

38. Tumuluru, J.S., C.C. Conner, and A.N. Hoover. 2016. "Method to produce durable pellets at lower energy consumption using high moisture corn stover and a corn starch binder in a flat die pellet mill." Journal of Visualized Experiments 112. https://doi.org/10.3791/54092.

39. Tumuluru, J.S. 2017. "Biomass Engineering, Size Reduction, Drying and Densification of High-Moisture Biomass." Presented at the DOE Bioenergy Technologies Office Project Peer Review, Denver, Colorado, March 7, 2017.

40. Tao, L., D. Schell, R. Davis, E. Tan, R. Elander, and A. Bratis. 2014. NREL 2012 Achievement of Ethanol Cost Targets: Biochemical Ethanol Fermentation via Dilute-Acid Pretreatment and Enzymatic Hydrolysis of Corn Stover. NREL/TP-5100-61563. Golden, CO: National Renewable Energy Laboratory.

41. Chen, Xiaowen, Erik Kuhn, Edward W. Jennings, Robert Nelson, Ling Tao, Min Zhang, and Melvin P. Tucker. 2016. "DMR (deacetylation and mechanical refining) processing of corn stover achieves high monomeric sugar concentrations $(230 \mathrm{~g} \mathrm{~L}-1)$ during enzymatic hydrolysis and high ethanol concentrations $(>10 \% \mathrm{v} / \mathrm{v})$ during fermentation without hydrolysate purification or concentration." Energy \& Environmental Science 9, no. 4: 1237-1245. https://doi.org/10.1039/C5EE03718B.

42. Chen, Xiaowen, Joseph Shekiro, Thomas Pschorn, Marc Sabourin, Melvin P. Tucker, and Ling Tao. 2015. "Techno-economic analysis of the deacetylation and disk refining process: characterizing the effect of refining energy and enzyme usage on minimum sugar selling price and minimum ethanol selling price." Biotechnology for Biofuels 8, no. 1: 173. https://dx.doi.org/10.1186\%2Fs 13068-0150358-0. 
43. Chen, X., et al. 2014. "Produce $2 \mathrm{~kg}$ each of 3 different deacetylated/mechanically refined/enzyme digested lignin rich corn stover residues for a joint milestone with the Lignin Utilization task for analysis and depolymerization." NREL Milestone Report.

44. Chen, Xiaowen, Erik Kuhn, Nick Nagle, Rob Nelson, Ling Tao, Nathan Crawford, and Melvin Tucker. 2018. "Recycling of Dilute Deacetylation Black Liquor to Enable Efficient Recovery and Reuse of Spent Chemicals and Biomass Pretreatment Waste." Frontiers in Energy Research 6: 51. https://dx.doi.org/10.3389/fenrg.2018.00051

45. Perry, R.H., D.W. Green, and J.O. Maloney. 1997. Perry's Chemical Engineers' Handbook, 7th Edition. New York: McGraw-Hill.

46. Bowen, E., S.C. Kennedy, and K. Miranda. 2010. "Ethanol from Sugar Beets: A Process and Economic Analysis." For the Degree of Bachelor of Science, Worcester Polytechnic Institute.

47. Stickel, Jonathan J., Birendra Adhikari, David A. Sievers, and John Pellegrino. 2017. "Continuous enzymatic hydrolysis of lignocellulosic biomass in a membrane-reactor system." Journal of Chemical Technology and Biotechnology 93, no. 8: 2181-2190. https://doi.org/10.1002/jctb.5559.

48. Fagerson, I. S. 1969. "Thermal degradation of carbohydrates; a review." Journal of Agricultural and Food Chemistry 17, no. 4: 747-750. https://doi.org/10.1021/jf60164a019.

49. Meerman, H.J., A.S. Kelley, and M. Ward. 2004. "Advances in Protein Expression in Filamentous Fungi." In Protein Expression Technologies: Current Status and Future Trends, edited by F. Baneyx. Norfolk, UK: Horizon Bioscience.

50. Warzywoda, M., et al., U.S. Patent No. 4,762,788, 28 November 1984.

51. Emme, B., Personal communication with D. Humbird. 2009.

52. Atkinson, B. and F. Mavituna. 1991. Biochemical Engineering and Biotechnology Handbook. New York: Stockton Press.

53. Schell, D., C. Riley, P. Bergeron, and P. Walter. 1991. Technical and Economic Analysis of an Enzymatic Hydrolysis Based Ethanol Plant. SERI/TP-232-4295. Golden, CO: Solar Energy Research Institute.

54. Humbird, D., et al. 2015. "Aeration cost and performance in STR and BC." NREL milestone report.

55. McMillan, J., et al. 2014. "Assessment of large-scale aeration economics." NREL milestone report.

56. Crater, J., C. Galleher, and J. Lievense. 2017. Consultancy on Large-Scale Submerged Aerobic Cultivation Process Design - Final Technical Report. NREL/SR-5100-67963. Golden, CO: National Renewable Energy Laboratory. 
57. Ledesma-Amaro, Rodrigo, Remi Dulermo, Xochitl Niehus, and Jean-Marc Nicauda. 2016. "Combining metabolic engineering and process optimization to improve production and secretion of fatty acids." Metabolic Engineering 38: 38-46. https://doi.org/10.1016/j.ymben.2016.06.004.

58. Shumaker, Andrew M., Bernardo M. da Costa, Kevin Holden, Louis G. Hom, Tarah S. Baron, and Noah Helman. 2018. "Enzyme variants with improved ester synthase properties." U.S. Patent No. 9879239B2.

59. Qiao, Kangjian, Thomas M. Wasylenko, Kang Zhou, Peng Xu, and Gregory Stephanopoulos. 2017. "Lipid production in Yarrowia lipolytica is maximized by engineering cytosolic redox metabolism." Nature Biotechnology 35, no.2: 173. https://doi.org/10.1038/nbt.3763.

60. Meadows, Adam L., Kristy M. Hawkins, Yoseph Tsegaye, and Annie E. Tsong. 2016. "Rewriting yeast central carbon metabolism for industrial isoprenoid production." Nature 537, no. 7622: 694. http://dx.doi.org/10.1038/nature19769.

61. Zhang, M., et al. 2015. "Down select best gene combination to demonstrate 2,3-BDO production at $10 \mathrm{~g} / \mathrm{L}$ in Zymomonas mobilis from glucose and xylose." NREL Milestone Report.

62. Zhang, M., et al. 2016. "Demonstrate production of 2,3 butanediol at $20 \mathrm{~g} / \mathrm{L}$ using engineered Zymomonas mobilis from glucose and xylose derived from DMR-EH biomass hydrolysate." NREL Milestone Report.

63. Zhang, M., Y.-C. Chou, and A. Mohagheghi. 2017. "Demonstrate production of 2,3-butanediol from glucose \& xylose at $35 \mathrm{~g} / \mathrm{L}$ using engineered Zymomonas mobilis using batch and/or fed-batch fermentations." NREL Milestone Report.

64. Davis, R., M. Biddy, and L. Tao. 2017. "Catalytic Pathway Alternatives: Conduct TEA modeling on at least two additional pathways for catalytic upgrading of hydrolysate intermediates, beyond the 'catalytic conversion of sugars' design case focused on APR." NREL Milestone Report.

65. Davis, R., et al. 2017. "Options to support \$2/GGE." NERL Milestone Report.

66. Davis, R., L. Tao, C. Scarlata, E.C.D. Tan, J. Ross, J. Lukas, and D. Sexton. 2015. Process Design and Economics for the Conversion of Lignocellulosic Biomass to Hydrocarbons Dilute-Acid and Enzymatic Deconstruction of Biomass to Sugars and Catalytic Conversion of Sugars to Hydrocarbons. NREL/TP-5100-62498. Golden, CO: National Renewable Energy Laboratory.

67. Ji, X.-J., H. Huang, and P.-K. Ouyang. 2011. "Microbial 2,3-butanediol production: A state-ofthe-art review." Biotechnology Advances 29, no. 3: 351-364.

https://doi.org/10.1016/j.biotechadv.2011.01.007.

68. Xiu, Z.-L. and A.-P. Zeng. 2008. "Present state and perspective of downstream processing of biologically produced 1,3-propanediol and 2,3-butanediol." Applied Microbiology and Biotechnology 78, no. 6: 917-926. https://doi.org/10.1007/s00253-008-1387-4.

69. $\quad$ Davis, R., et al. 2016. "TEA Identification of Most Promising Options for 2022 Targets." NREL milestone report. 
70. Narula, Chaitanya, Zhenglong Li, Erik Casbeer, Robert Geiger, Melanie Moses-Debusk, Martin Keller, Michelle Buchanan, and Brian Davison. 2015. "Heterobimetallic zeolite, InV-ZSM-5, enables efficient conversion of biomass derived ethanol to renewable hydrocarbons." Scientific Reports 5: 16039. http://dx.doi.org/10.1038/srep16039.

71. Li, Zhenglong, Oak Ridge National Laboratory, personal communication, 2018.

72. Zheng, Quanxing, Michael D. Wales, Michael G. Heidlage, Mary Rezac, Hongwang Wang, Stefan H. Bossmann, and Keith L. Hohn. 2015. "Conversion of 2, 3-butanediol to butenes over bifunctional catalysts in a single reactor." Journal of Catalysis 330: 222-237. https://doi.org/10.1016/j.jcat.2015.07.004.

73. Twigg, M.V. 1989. Catalyst Handbook, edited by M.V. Twigg. London: Wolfe.

74. Zhang, Xin, Jin Zhong, Jianwei Wang, Linhui Zhang, Junkui Gao, and Aisong Liub. 2009. "Catalytic performance and characterization of Ni-doped HZSM-5 catalysts for selective trimerization of n-butene." Fuel Processing Technology 90, no. 7-8: 863-870. https://doi.org/10.1016/j.fuproc.2009.04.011.

75. Golombok, M., and J. De Bruijn. 2000. "Density Variations in a Reactor During Liquid Full Dimerization." Chemical Engineering Research and Design 78, no. 1: 145-147. https://doi.org/10.1205/026387600526988.

76. Golombok, M., and J. de Bruijn. 2001. "Catalysts for producing high octane-blending value olefins for gasoline." Applied Catalysis A: General 208, no. 1-2: 47-53. https://doi.org/10.1016/S0926-860X(00)00684-0.

77. Wright, M.E., B.G. Harvey, and R.L. Quintana. 2015. "Diesel and Jet Fuels Based on the Oligomerization of Butene." U.S. Patent No. 9,181,144.

78. Blommel, Paul, Brice Dally, Warren Lyman, and Randy Cortright. "Method and systems for making distillate fuels from biomass." International Patent Application No. WO2012109241.

79. Choi, Kieun, Byoung Seung Jeon, Byung-Chun Kim, Min-Kyu Oh, Youngsoon Um, and Byoung-In Sang. 2013. "In situ biphasic extractive fermentation for hexanoic acid production from sucrose by Megasphaera elsdenii NCIMB 702410." Applied Biochemistry and Biotechnology 171, no. 5: 1094-1107. https://doi.org/10.1007/s12010-013-0310-3.

80. Jiang, X.-1., S. Yong, and W.-y. Zhu. 2016. "Fermentation characteristics of Megasphaera elsdenii J6 derived from pig feces on different lactate isomers." Journal of Integrative Agriculture 15, no. 7: 1575-1583. https://doi.org/10.1016/S2095-3119(15)61236-9.

81. Hashizume, Kenta, Takamitsu Tsukahara, Kouji Yamada, Hironari Koyama, and Kazunari Ushida. 2003. "Megasphaera elsdenii JCM1772T normalizes hyperlactate production in the large intestine of fructooligosaccharide-fed rats by stimulating butyrate production." The Journal of Nutrition 133, no. 10: 3187-3190. https://doi.org/10.1093/jn/133.10.3187. 
82. Roddick, F.A. and M.L. Britz. 1997. "Production of hexanoic acid by free and immobilised cells of Megasphaera elsdenii: influence of in-situ product removal using ion exchange resin. Journal of Chemical Technology \& Biotechnology 69, no. 3: 383-391. https://doi.org/10.1002/(SICI)10974660(199707)69:3<383::AID-JCTB723>3.0.CO;2-H.

83. Nelson, Robert S, Darren J. Peterson, Eric M. Karp, Gregg T. Beckham, and Davinia Salvachúa. 2017. "Mixed carboxylic acid production by Megasphaera elsdenii from glucose and lignocellulosic hydrolysate." Fermentation 3, no. 1: 10. https://doi.org/10.3390/fermentation3010010.

84. Lee, Joungmin, Yu-Sin Jang, Mee-Jung Han, Jin Young Kim, and Sang Yup Lee. 2016. "Deciphering Clostridium tyrobutyricum metabolism based on the whole-genome sequence and proteome analyses." MBio 7, no. 3: e00743-16. https://doi.org/10.1128/mBio.00743-16.

85. Liu, X., Y. Zhu, and S.-T. Yang. 2006. "Butyric acid and hydrogen production by Clostridium tyrobutyricum ATCC 25755 and mutants." Enzyme and Microbial Technology 38 no. 3-4: 521-528. https://doi.org/10.1016/j.enzmictec.2005.07.008.

86. Zhu, Y., and S.-T. Yang. 2004. "Effect of $\mathrm{pH}$ on metabolic pathway shift in fermentation of xylose by Clostridium tyrobutyricum." Journal of Biotechnology 110, no. 2: 143-157. https://doi.org/10.1016/j.jbiotec.2004.02.006.

87. Russell, J. 1992. "Another explanation for the toxicity of fermentation acids at low pH: anion accumulation versus uncoupling." Journal of Applied Microbiology 73, no. 5: 363-370. https://doi.org/10.1111/j.1365-2672.1992.tb04990.x.

88. Zigová, Jana, Ernest Šturdík, Dušan Vandák, and Štefan Schlosser. 1999. "Butyric acid production by Clostridium butyricum with integrated extraction and pertraction." Process Biochemistry 34, no. 8: 835-843. https://doi.org/10.1016/S0032-9592(99)00007-2.

89. Kawabata, N., J.-i. Yoshida, and Y. Tanigawa. 1981. "Removal and recovery of organic pollutants from aquatic environment. 4. Separation of carboxylic acids from aqueous solution using crosslinked poly (4-vinylpyridine)." Industrial \& Engineering Chemistry Product Research and Development 20, no. 2: 386-390. https://doi.org/10.1021/i300002a030.

90. Kertes, A., and C.J. King. 1986. "Extraction chemistry of fermentation product carboxylic acids." Biotechnology and Bioengineering 28, no. 2: 269-282. https://doi.org/10.1002/bit.260280217.

91. Schlosser, Š., R. Kertész, and J. Martak. 2005. "Recovery and separation of organic acids by membrane-based solvent extraction and pertraction: An overview with a case study on recovery of MPCA." Separation and Purification Technology 41, no. 3: 237-266. https://doi.org/10.1016/j.seppur.2004.07.019.

92. Um, B.-H., B. Friedman, and G.P. van Walsum. 2011. "Conditioning hardwood-derived prepulping extracts for use in fermentation through removal and recovery of acetic acid using trioctylphosphine oxide (TOPO)." Holzforschung 65, no. 1: 51-58. https://doi.org/10.1515/hf.2010.115. 
93. Wasewar, Kailas L., Archis A. Yawalkar, Jacob A. Moulijn, and Vishwas G. Pangarkar. 2004. "Fermentation of glucose to lactic acid coupled with reactive extraction: a review." Industrial \& Engineering Chemistry Research 43, no. 19: 5969-5982. https://doi.org/10.1021/ie049963n.

94. Zhang, Chunhui, Hua Yang, Fangxiao Yang, and Yujiu Ma. 2009. "Current progress on butyric acid production by fermentation." Current Microbiology 59, no. 6: 656-663. https://doi.org/10.1007/s00284-009-9491-y.

95. Papoutsakis, E.T. 1984. "Equations and calculations for fermentations of butyric acid bacteria." Biotechnology and Bioengineering 26, no. 2: 174-187. https://doi.org/10.1002/bit.260260210.

96. Hano, Tadashi, Michiaki Matsumoto, Takaaki Ohtake, Kiyotaka Sasaki, Fumiaki Hori, and Yoshinobu Kawano. 1990. "Extraction equilibria of organic acids with tri- $n$-octylphosphineoxide." Journal of Chemical Engineering of Japan. 23, no. 6: 734-738. https://doi.org/10.1252/jcej.23.734.

97. Matsumoto, Michiaki, Shinji Uenoyama, Tadashi Hano, Makato Hirata, and Shigenobu Miura. 1996. "Extraction Kinetics of Organic Acids with Tri- $n$-octylphosphine Oxide." Journal of Chemical Technology \& Biotechnology 67, no. 3: 260-264. https://doi.org/10.1002/(SICI)10974660(199611)67:3<260::AID-JCTB559>3.0.CO;2-Y.

98. Saboe, Patrick O., Lorenz P. Manker, William E. Michener, Darren J. Peterson, David G. Brandner, Stephen P. Deutch, Manish Kumar, Robin M. Cywar, Gregg T. Beckham, and Eric M. Karp. 2018. "In situ recovery of bio-based carboxylic acids." Green Chemistry 20, no. 8: 1791-1804. https://doi.org/10.1039/C7GC03747C.

99. Shylesh, Sankaranarayanapillai, Amit A. Gokhale, Keyang Sun, Adam M. Grippo, Deepak Jadhav, Alice Yeh, Christopher R. Hob, and Alexis T. Bell. 2017. "Integrated catalytic sequences for catalytic upgrading of bio-derived carboxylic acids to fuels, lubricants and chemical feedstocks." Sustainable Energy \& Fuels 1, no. 8: 1805-1809. https://doi.org/10.1039/C7SE00359E.

100. Pham, Tu N., Tawan Sooknoi, Steven P. Crossley, and Daniel E. Resasco. 2013. "Ketonization of carboxylic acids: mechanisms, catalysts, and implications for biomass conversion." ACS Catalysis 3, no. 11: 2456-2473. https://doi.org/10.1021/cs400501h.

101. Moore, Cameron M., Rhodri W. Jenkins, Michael T. Janicke, William L. Kubic Jr., Evgueni Polikarpov, Troy A. Semelsberger, and Andrew D. Sutton. 2016. "Synthesis of Acetone-Derived C6, C9, and C12 Carbon Scaffolds for Chemical and Fuel Applications." ChemSusChem 9, no. 24: 33823386. https://doi.org/10.1002/cssc.201600936.

102. Sacia, Eric R., Madhesan Balakrishnan, Matthew H. Deaner, Konstantinos A. Goulas, F. Dean Toste, and Alexis T. Bell. 2015. "Highly Selective Condensation of Biomass-Derived Methyl Ketones as a Source of Aviation Fuel." ChemSusChem 8, no. 10: 1726-1736. https://doi.org/10.1002/cssc.201500002.

103. Aspen Technology. 2018. "Aspen Capital Cost Estimator." https://www.aspentech.com/en/products/engineering/aspen-capital-cost-estimator. 
104. Datta, Saurav, Yupo J. Lin, Daniel J. Schell, C. S. Millard, Sabeen F. Ahmad, Michael P. Henry, P. Gillenwater, Anthony T. Fracaro, A. Moradia, Zofia P. Gwarnicki, and Seth W. Snyder. 2013. "Removal of Acidic Impurities from Corn Stover Hydrolysate Liquor by Resin Wafer Based Electrodeionization." Industrial \& Engineering Chemistry Research 52, no. 38: 13777-13784. https://doi.org/10.1021/ie4017754.

105. Arora, M. B., J. A. Hestekin, S. W. Snyder, E. J. St. Martin, Y. J. Lin, M. I. Donnelly, and C. Sanville Millard. 2007. "The Separative Bioreactor: A Continuous Separation Process for the Simultaneous Production and Direct Capture of Organic Acids." Sep Sci Technol 42, no. 11: 25192538. https://doi.org/10.1080/01496390701477238.

106. Schutyser, W., T. Renders, S. Van den Bosch, S.-F. Koelewijn, G. T. Beckham, and B. F. Sels. 2018. "Chemicals from lignin: an interplay of lignocellulose fractionation, depolymerisation, and upgrading." Chemical Society Reviews 47, no. 3: 852-908. https://doi.org/10.1039/C7CS00566K.

107. Beckham, Gregg T., Christopher W. Johnson, Eric M. Karp, Davinia Salvachúa, and Derek R. Vardon. 2016. "Opportunities and challenges in biological lignin valorization." Current Opinion in Biotechnology 42: 40-53. https://doi.org/10.1016/j.copbio.2016.02.030.

108. Pavone, A. 2012. Bio-Based Adipic Acid. Santa Clara, CA: IHS Chemical.

109. Kojima, Yutaka, Hiroshi Fujisawa, Atsushi Nakazawa, Teruko Nakazawa, Fuminori Kanetsuna, Hiroshi Taniuchi, Mitsuhiro Nozaki, and Osamu Hayaishi. 1967 "Studies on Pyrocatechase I. Purification and Spectral Properties." Journal of Biological Chemistry 242, no. 14: 3270-3278.

110. Nakazawa, T. 2002. "Travels of a Pseudomonas from Japan around the world." Environmental Microbiology 4, no. 12: 782-786. http://dx.doi.org/10.1046/j.1462-2920.2002.00310.x.

111. Williams, P.A., and K. Murray. 1974. "Metabolism of benzoate and the methylbenzoates by Pseudomonas putida (arvilla) mt-2: evidence for the existence of a TOL plasmid." Journal of Bacteriology 120, no. 1: 416-423.

112. Nelson, K.E., C. Weinel, I.T. Paulsen, R.J. Dodson, H. Hilbert, V.A. Martins dos Santos, and D.E. Fouts, et al. 2002. "Complete genome sequence and comparative analysis of the metabolically versatile Pseudomonas putida KT2440." Environmental Microbiology 4, no. 12: 799-808. https://doi.org/10.1046/j.1462-2920.2002.00366.x.

113. Belda, Eugeni, Ruben G. A. van Heck, Maria José Lopez-Sanchez, Stéphane Cruveiller, Valérie Barbe, Claire Fraser, and Hans-Peter Klenk, et al. 2016. "The revisited genome of Pseudomonas putida KT2440 enlightens its value as a robust metabolic chassis." Environmental Microbiology 18 no. 10: 3403-3424. https://doi.org/10.1111/1462-2920.13230.

114. Linger, Jeffrey G., Derek R. Vardon, Michael T. Guarnieri, Eric M. Karp, Glendon B. Hunsinger, Mary Ann Franden, and Christopher W. Johnson, et al. 2014. "Lignin valorization through integrated biological funneling and chemical catalysis." Proceedings of the National Academy of Sciences 111, no. 33: 12013-12018. https://doi.org/10.1073/pnas.1410657111. 
115. Thompson, B., M. Machas, and D.R. Nielsen. 2015. "Creating pathways towards aromatic building blocks and fine chemicals." Current Opinion in Biotechnology 36: 1-7.

https://doi.org/10.1016/j.copbio.2015.07.004.

116. Burgard, A.P., P. Pharkya, and R.E. Osterhout. 2012. "Microorganisms for the production of adipic acid and other compounds." U.S. Patent No. 7799545B2.

117. Frost, J.W., and K.M. Draths. 1996. "Synthesis of adipic acid from biomass-derived carbon sources." U.S Patent No. 5487987A.

118. Frost, J.W., and K.M. Draths. 1997. "Bacterial cell tranformants for production of cis, cismuconic acid and catechol." U.S. Patent No. 5616496A.

119. Niu, W., K. Draths, and J. Frost. 2002. "Benzene-Free Synthesis of Adipic Acid."

Biotechnology Progress 18, no. 2: 201-211. https://doi.org/10.1021/bp010179x.

120. Yu, J.L., X.X. Xia, J.J. Zhong, and Z.G. Qian. 2014. "Direct biosynthesis of adipic acid from a synthetic pathway in recombinant Escherichia coli." Biotechnology and Bioengineering 111. no. 12: 2580-2586. https://doi.org/10.1002/bit.25293.

121. Johnson, Christopher W., Davinia Salvachúa, Payal Khanna, Holly Smith, Darren J. Peterson, and Gregg T. Beckham. 2016. "Enhancing muconic acid production from glucose and lignin-derived aromatic compounds via increased protocatechuate decarboxylase activity." Metabolic Engineering Communications 3: 111-119. https://doi.org/10.1016/j.meteno.2016.04.002.

122. Vardon, Derek R., Nicholas A. Rorrer, Davinia Salvachúa, Amy E. Settle, Christopher W. Johnson, Martin J. Menart, and Nicholas S. Cleveland, et al. 2016. "cis, cis-Muconic acid: separation and catalysis to bio-adipic acid for nylon-6, 6 polymerization." Green Chemistry 18, no. 11: $3397-$ 3413. https://doi.org/10.1039/C5GC02844B.

123. Vardon, Derek R., Mary Ann Franden, Christopher W. Johnson, Eric M. Karp, Michael T. Guarnieri, Jeffrey G. Linger, Michael J. Salm, Timothy J. Strathmann, and Gregg T. Beckham. 2015. "Adipic acid production from lignin." Energy \& Environmental Science 8, no. 2: 617-628. https://doi.org/10.1039/C4EE03230F.

124. Löwe, Hannes, Lukas Schmauder, Karina Hobmeier, Andreas Kremling, and Katharina Pflüger-Grau. 2017. "Metabolic engineering to expand the substrate spectrum of Pseudomonas putida toward sucrose." MicrobiologyOpen 6, no. 4. https://doi.org/10.1002/mbo3.473.

125. Orth, J.D., I. Thiele, and B.Ø. Palsson. 2010. "What is flux balance analysis?" Nature Biotechnology 28, no. 3: 245-248. https://doi.org/10.1038/nbt.1614.

126. Puchałka, J., et al. 2008. "Genome-scale reconstruction and analysis of the Pseudomonas putida KT2440 metabolic network facilitates applications in biotechnology." PLoS computational biology 4, no. 10: p. e1000210. https://doi.org/10.1371/journal.pcbi.1000210.

127. Jayakody, Lahiru N., Christopher W. Johnson, Jason M. Whitham, Richard J. Giannone, Brenna A. Black, Nicholas S. Cleveland, and Dawn M. Klingeman, et al. 2018. "Thermochemical 
wastewater valorization via enhanced microbial toxicity tolerance." Energy \& Environmental Science 11, no. 6: 1625-1638. https://doi.org/10.1039/C8EE00460A.

128. Shiloach, J., and R. Fass. 2005. "Growing E. coli to high cell density-a historical perspective on method development." Biotechnology Advances 23, no. 5: 345-357.

https://doi.org/10.1016/j.biotechadv.2005.04.004.

129. Van't Riet, K., and J. Tramper. 1991. Basic Bioreactor Design. Boca Raton, FL: CRC Press.

130. Gaivoronskii, A., and V. Granzhan. 2005. "Solubility of adipic acid in organic solvents and water." Russian Journal of Applied Chemistry 78, no. 3: https://doi.org/404-408. 10.1007/s11167-0050305-0.

131. Scelfo, S., R. Pirone, and N. Russo. 2016. "Thermodynamics of cis, cis-muconic acid solubility in various polar solvents at low temperature range." Journal of Molecular Liquids 222: 823-827. https://doi.org/10.1016/j.molliq.2016.07.129.

132. Li, Qiang, DanWang, YongWu, Wangliang Li, Yunjian Zhang, Jianmin, Xing, and Zhiguo Su. 2010. "One step recovery of succinic acid from fermentation broths by crystallization." Separation and Purification Technology 72, no. 3: 294-300. https://doi.org/10.1016/j.seppur.2010.02.021.

133. Urbanus, J., C.P.M. Roelands, D. Verdoes, J.H. ter Horst. 2012. "Intensified crystallization in complex media: Heuristics for crystallization of platform chemicals." Chemical Engineering Science 77: 18-25. https://doi.org/10.1016/j.ces.2012.02.019.

134. Gary, J.H., G.E. Handwerk, and M.J. Kaiser. 2007. Petroleum Refining: Technology and Economics. Boca Raton, FL: CRC Press.

135. Nielsen, R.H. 2007. Process Economics Program Report 214A: Heavy Oil Hydrotreating. Menlo Park, CA: SRI Consulting.

136. Kaiser, M.J., and J.H. Gary. 2007. "Study updates refinery investment cost curves." Oil \& Gas Journal, April 23, 2007. https://www.ogj.com/articles/print/volume-105/issue-16/processing/studyupdates-refinery-investment-cost-curves.html.

137. Refining Process Services Inc. 2008. "Hydrotreating \& Hydrocracking Process Technology Seminar." Presented in Houston, Texas, in 2008.

138. Palmer, E., S. Polcar, and A. Wong. 2009. "Clean Diesel Hydrotreating." Petroleum Technology Quarterly January 2009.

139. Guthrie, K.M. 1974. Process Plant Estimating, Evaluation, and Control. Carlsbad, CA: Craftsman Book Company of America.

140. Seider, W.D., J. Seader, and D.R. Lewin. 2004. Product and Process Design Principles. New York: Wiley. 
141. Bray, R.G. 2007. Process Economics Program Report 251A: Advances in Biodeisel and Renweable Diesel Production. London: IHS Consulting.

142. Cesar, M. 2006. Process Economics Program: Advances in Paraxylene Technology. London: IHS Consulting.

143. Salvachúa, Davinia, Eric M. Karp, Claire T. Nimlos, Derek R. Vardon, and Gregg T.

Beckham. 2015. "Towards lignin consolidated bioprocessing: simultaneous lignin depolymerization and product generation by bacteria." Green Chemistry 17, no. 11: 4951-4967.

https://doi.org/10.1039/C5GC01165E

144. Rinaldi, Robert, Robin Jastrzebski, Matthew T. Clough, John Ralph, Marco Kennema, Pieter C. A. Bruijnincx, and Bert M. Weckhuysen. 2016. "Paving the way for lignin valorisation: recent advances in bioengineering, biorefining and catalysis." Angewandte Chemie International Edition 55, no. 29: 8164-8215. https://doi.org/10.1002/anie.201510351.

145. Ragauskas, Arthur J., Gregg T. Beckham, Mary J. Biddy, Richard Chandra, Fang Chen, Mark F. Davis, and Brian H. Davison, et al. 2014. "Lignin valorization: improving lignin processing in the biorefinery." Science 344, no. 6185: 1246843. https://doi.org/10.1126/science.1246843

146. U.S. Department of Energy. 2016. Bioenergy Technologies Office Multi-Year Program Plan. Washington, DC: U.S. Department of Energy.

147. Chemical Engineering Magazine. 2011. "Chemical Engineering Magazine Plant Cost Index." http://folk.ntnu.no/magnehi/cepci_2011_py.pdf.

148. U.S. Bureau of Labor Statistics. 2009. "National Employment, Hours, and Earnings Catalog, Industry: Chemicals and Allied Products, 1980-2009." http://data.bls.gov/cgi-bin/srgate.

149. U.S. Department of Agriculture Economic Research Service. 2018. "Table 7-U.S. wholesale list price for glucose syrup, Midwest markets, monthly, quarterly, and by calendar and fiscal year." 2018. https://www.ers.usda.gov/data-products/sugar-and-sweeteners-yearbook-tables.aspx.

150. U.S. Energy Information Administration. 2015. "Electricity Data Browser-Average Retail Price of Electricity." https://www.eia.gov/electricity/data/browser/.

151. Dillich, S., T. Ramsden, and M. Melina. 2012. "Hydrogen Production Cost Using Low-Cost Natural Gas." DOE Hydrogen and Fuel Cells Program Record \#12024. http://www.hydrogen.energy.gov/pdfs/12024_h2_production_cost_natural_gas.pdf

152. Steinwinder, Thomas, Everett Gill, and Matthew Gerhardt. 2012. Process Design of Wastewater Treatment for the NREL Cellulosic Ethanol Model. NREL/SR-5100-51838. Golden, CO: National Renewable Energy Laboratory.

153. Peters, Max, Klaus Timmerhaus, and Ronald West. 2003. Plant Design and Economics for Chemical Engineers. New York: McGraw-Hill. 
154. SRI Consulting. 2008. "U.S. Producer Price Indexes - Chemicals and Allied Products/Industrial Inorganic Chemicals Index." In Chemical Economics Handbook. Menlo Park, CA: SRI Consulting.

155. Short, W., D.J. Packey, and T. Holt. 1995. A Manual for the Economic Evaluation and Energy Efficiency and Renewable Energy Technologies. NREL/TP-462-5173. Golden, CO: National Renewable Energy Laboratory.

156. Gary, J.H., and G.E. Handwerk. 1994. Petroleum Refining: Technology and Economics, 3rd Edition. New York: Marcel Dekker.

157. Biddy, Mary J., Ryan Davis, David Humbird, Ling Tao, Nancy Dowe, Michael T. Guarnieri, and Jeffrey G. Linger, et al. 2016. "The techno-economic basis for coproduct manufacturing to enable hydrocarbon fuel production from lignocellulosic biomass." ACS Sustainable Chemistry \& Engineering 4, no. 6: 3196-3211. https://doi.org/10.1021/acssuschemeng.6b00243

158. Cai, Hao, Jeongwoo Han, Michael Wang, Ryan Davis, Mary Biddy, and Eric Tan. 2018. "Lifecycle analysis of integrated biorefineries with co-production of biofuels and bio-based chemicals: coproduct handling methods and implications." Biofuels, Bioproducts and Biorefining 12, no. 5. https://doi.org/10.1002/bbb.1893.

159. Munasinghe, P.C., and S.K. Khanal. 2010. "Biomass-derived syngas fermentation into biofuels: Opportunities and challenges." Bioresource Technology 101, no. 13: 5013-5022. https://doi.org/10.1016/j.biortech.2009.12.098.

160. Fei, Qiang, Aaron W. Puri, Holly Smith, Nancy Dowe, and Philip T. Pienkos. 2018. "Enhanced biological fixation of methane for microbial lipid production by recombinant Methylomicrobium buryatense." Biotechnology for Biofuels 11, no. 129: 1-11. https://doi.org/10.1186/s13068-018-1128-6.

161. Henard, C.A., and M.T. Guarnieri. 2016. "Metabolic and techno-economic assessment of highpotential hydrocarbon targets from methane, down-selecting two targets for FY17 efforts." NREL Milestone Report.

162. Anderson, Eric M., Rui Katahira, Michelle Reed, Michael G. Resch, Eric M. Karp, Gregg T. Beckham, and Yuriy Román-Leshkov. 2016. "Reductive catalytic fractionation of corn stover lignin." ACS Sustainable Chemistry \& Engineering 4, no. 12: 6940-6950. https://doi.org/10.1021/acssuschemeng.6b01858.

163. Anderson, Eric M., Michael L. Stone, Rui Katahira, Michelle Reed, Gregg T. Beckham, and Yuriy Román-Leshkov. 2017. "Flowthrough Reductive Catalytic Fractionation of Biomass." Joule 1, no. 3: 613-622. https://doi.org/10.1016/j.joule.2017.10.004.

164. Laskar, Dhrubojyoti D., Melvin P. Tucker, Xiaowen Chen, Gregory L. Helms, and Bin Yang. 2014. "Noble-metal catalyzed hydrodeoxygenation of biomass-derived lignin to aromatic hydrocarbons." Green Chemistry 16, no. 2: 897-910. https://doi.org/10.1039/C3GC42041H.

165. Agile BioFoundry. 2018. "Agile BioFoundry Home Page." https://agilebiofoundry.org/. 


\section{Appendix A. Individual Equipment Costs Summary}

The following table shows abbreviated specifications, purchased cost, and installed cost for each piece of equipment in this process design. Although each piece of equipment has its own line, many were quoted as part of a package, so their scaling calculations are not shown. NREL would like to acknowledge the subcontractors and equipment vendors who assisted us with cost estimates over recent years as were utilized for this design report. 
Acids Pathway

\begin{tabular}{|c|c|c|c|c|c|c|c|c|c|c|c|c|c|c|c|c|c|}
\hline \multirow{2}{*}{\begin{tabular}{|c|} 
A200: Pretreatment \\
EQUIPMENT TITLE \\
\end{tabular}} & \multicolumn{5}{|c|}{ Mechanical Equipment List } & \multicolumn{12}{|c|}{ Scaled Installed Costs } \\
\hline & DESCRIPTION & HP & MATERIAL & 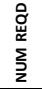 & $\$$ & 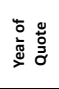 & $\begin{array}{c}\text { Purch Cost in } \\
\text { Base Yr }\end{array}$ & Scaling Variable & 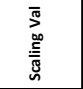 & $\frac{\mathscr{n}}{5}$ & 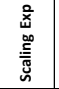 & 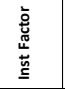 & 㧝 & 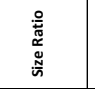 & $\begin{array}{c}\text { Scaled Purch } \\
\text { cost }\end{array}$ & $\begin{array}{l}\text { Purch Cost in } \\
\text { Proj year }\end{array}$ & $\begin{array}{l}\text { Inst Cost in } \\
\text { Proj year }\end{array}$ \\
\hline Flash Tank Agitator & Side-mounted, $3 \times 75$ hp. $(170 \mathrm{~kW})$ & $170 \mathrm{~kW}$ & 316 LSS & 3 & $\$ 90,000$ & 2009 & \multicolumn{2}{|c|}{$\begin{array}{l}\$ 90,000 \text { strm.a200.254 } \\
\$ 2.900 \text { strm } .200 .228\end{array}$} & 252891 & $\mathrm{~kg} / \mathrm{hr}$ & 0.50 & & 192,146 & 0.76 & $\$ 78,450$ & $\$ 81,426$ & $\$ 122,139$ \\
\hline $\begin{array}{l}\text { Ammonia Addition Tank Agitator } \\
\text { Ammoni Static Mixer }\end{array}$ & & $10 \mathrm{hp}$ & ss & 1 & $\begin{array}{ll}\$ 21,900 \\
\$ 5500\end{array}$ & 2009 & \multirow{3}{*}{\multicolumn{2}{|c|}{ 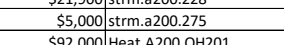 }} & $\begin{array}{ll}410369 \\
157788\end{array}$ & $\mathrm{~kg} / \mathrm{hr}$ & 0.50 & & $\begin{array}{l}230,647 \\
38500\end{array}$ & 0.56 & 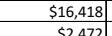 & \begin{tabular}{|l|l|l}
$\$ 17,041$ \\
5566 \\
\end{tabular} & $\begin{array}{ll}\$ \$ 25,562 \\
\$ 566\end{array}$ \\
\hline Ammonia Static Mixer & 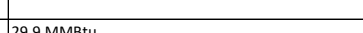 & & $\frac{5 S}{30455}$ & 1 & $\begin{array}{l}\$ 5,000 \\
\$ 200\end{array}$ & 2009 & & & 157478 & $\mathrm{~kg} / \mathrm{hr}$ & 0.50 & & 38,500 & 0.2445 & $\begin{array}{ll}52,472 \\
5053\end{array}$ & $\begin{array}{ll}52,566 \\
5927\end{array}$ & $\begin{array}{ll}\$ 2,566 \\
\$ 2053\end{array}$ \\
\hline Pretreatment Water Heater & $29.9 \mathrm{MmBtu}$ & & 30455 & 1 & $\$ 92,000$ & 2010 & & & & Gcal/hr & 0.70 & & $(0.29)$ & 0.04 & $\$ 9,453$ & $\$ 9,297$ & $\$ 20,453$ \\
\hline $\begin{array}{l}\text { Pretreatment Sugar Beet extruder } \\
\text { Milling gavipment }\end{array}$ & & & & $\frac{1}{8}$ & $\begin{array}{l}\$ 5,424,000 \\
\$ 266700\end{array}$ & 2010 & \multicolumn{2}{|c|}{ 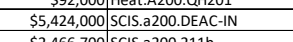 } & 38600 & $\mathrm{~kg} / \mathrm{hr}$ & 1.00 & 1.0 & $\begin{array}{l}70,483 \\
57206 \\
\end{array}$ & 1.83 & $\begin{array}{r}\$ 9,904,187 \\
\$ 1,72,601\end{array}$ & $\$ 9,740,555$ & $\begin{array}{r}\$ 9,740,555 \\
\$ 98,651\end{array}$ \\
\hline & $200 \mathrm{kw} / \mathrm{dry}$ ton & & & $\frac{8}{11}$ & $\$ \frac{\$ 2,466,700}{\$ 578,000}$ & $\frac{2013}{2013}$ & \multirow{2}{*}{\multicolumn{2}{|c|}{ 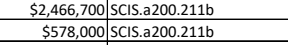 }} & $\frac{62942}{62942}$ & $\mathrm{~kg} / \mathrm{hr}$ & $0.60 \mid$ & & $\begin{array}{r}57,306 \\
57306 \\
\end{array}$ & 0.91 & $\begin{array}{l}\$ 19,733,600 \\
\$ 6338000\end{array}$ & 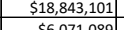 & $\frac{\$ 28,264,651}{\$ 8,90924}$ \\
\hline $\begin{array}{l}\text { |lilling Equipment-SSego Mill } \\
\text { Blowdown Tank Discharge Pump }\end{array}$ & 1900 GPM, 150 FT TDH & 125 & 31655 & $\frac{11}{1}$ & $\frac{\$ 587,000}{\$ 25,635}$ & $\frac{2013}{2010}$ & & & 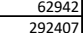 & $\mathrm{kg} / \mathrm{hr}$ & $\frac{0.60 \mid}{0.80}$ & $\begin{array}{r}1.4 \\
2.3 \\
\end{array}$ & $\frac{57,306}{193,44}$ & $\frac{0.91}{0.66}$ & $\frac{\$ 6,358,000}{\$ 18,39}$ & $\begin{array}{r}\$ 6,071,089 \\
\$ \$ 18,09 \\
\end{array}$ & $\begin{array}{r}\$ 8,499,524 \\
\$ \$ 1,614 \\
\end{array}$ \\
\hline $\begin{array}{l}\text { Blowdown Iank Discharge Pump } \\
\text { Flash Tank Discharge Pump }\end{array}$ & 900 GPM, 150 FT TDH & 75 & $\begin{array}{l}31565 s \\
3165 s \\
\end{array}$ & $\frac{1}{1}$ & $\begin{array}{l}\$ \$ 3,6,63 \\
\$ 30,000 \\
\end{array}$ & $\frac{2010}{2009}$ & \multicolumn{2}{|c|}{ 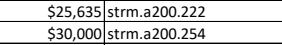 } & 204390 & $\begin{array}{ll}\mathrm{k} / \mathrm{g} / \mathrm{hr} \\
\mathrm{k} / \mathrm{hr}\end{array}$ & 0.80 & $\begin{array}{r}2.3 \\
2.3 \\
\end{array}$ & $\begin{array}{l}19,3,144 \\
192,146\end{array}$ & 0.06 & $\begin{aligned} & \$ 18,399 \\
& \$ 28,554 \\
&\end{aligned}$ & $\begin{array}{r}\$ 1,89,633 \\
\$ 237 \\
\end{array}$ & $\begin{array}{l}\$ 41,614 \\
\$ 68,165 \\
\end{array}$ \\
\hline Deacetylation Tank Discharge Pump & 1771 GPM, 150 FT TDH & 100 & $3165 \mathrm{~s}$ & 1 & $\$ 22,500$ & 2009 & \multirow{2}{*}{\multicolumn{2}{|c|}{$\begin{array}{l}22,500 \text { strm.a200.211a } \\
\$ 22,500 \text { strm.a200.228 }\end{array}$}} & 402194 & $\mathrm{~kg} / \mathrm{hr}$ & 0.80 & 2.3 & 283,665 & 0.71 & & & 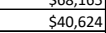 \\
\hline Hydrolyzate Pump & 1771 GPM, 150 FT TDH & 100 & 31655 & 1 & & 2009 & & & 402194 & & 0.80 & 2.3 & 230,647 & 0.57 & \$14,421 & \$14,968 & 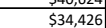 \\
\hline Deacetylation reactor conveyors & Feed and discharge drag conveyors & $40 \mathrm{hp}$ & s5316 & 3 & $\$ 110,000$ & 2013 & \multicolumn{2}{|c|}{$\begin{array}{l}\$ 2,5,500 \text { Strtm.a.200.228 } \\
\$ 110,000 \text { strm.a200.211a }\end{array}$} & & & 0.80 & 1.7 & 283,665 & 1.02 & $\$ 336,175$ & $\$ 321,004$ & $\$ 545,707$ \\
\hline S/L Split Discharge Pump to WWT & 900 GPM, 150 FT TDH & 75 & 31655 & 1 & $\$ 30,000$ & 2009 & \multirow{2}{*}{\multicolumn{2}{|c|}{$\begin{array}{l}\$ 30,000 \text { strm. } .2200 .4 \\
\$ 511,000 \\
\text { strm. } .200 .223 \\
\end{array}$}} & 204390 & $\mathrm{~kg} / \mathrm{hr}$ & 0.80 & $2.3 .3>3$ & 193,144 & 0.94 & $\$ 28,672$ & $\$ 29,760$ & $\$ 68,447$ \\
\hline Flash Tank & $23^{\prime} \times 48^{\prime}-110,000$ gal. & & SS3316 & 1 & $\$ 511,000$ & 2009 & & & 264116 & $\mathrm{~kg} / \mathrm{hr}$ & 0.70 & 2.0 & $\mid 193,144$ & 0.73 & $\$ 410,470$ & $\$ 426,042$ & $\$ 852,084$ \\
\hline \multirow{2}{*}{ Ammonia Addition Tank } & $118,000 \mathrm{gal}, \mathrm{hhr}$ residence time & & $5 S 304$ & 1 & $\$ 236,000$ & 2009 & $\$ 236,000$ & strm.a200.228 & 410369 & $\mathrm{~kg} / \mathrm{hr}$ & 0.70 & 2.0 & 230,647 & 0.56 & $\$ 157,671 \mid$ & $\$ 163,653$ & $\$ 327,306$ \\
\hline & & & & & & & & & & & & & \multicolumn{2}{|c|}{ Area 200 Totals } & $\$ 37,113,956$ & $\$ 35,785,895$ & $\$ 48,653,825$ \\
\hline A300: Hydrolysis and Fermentation & \multicolumn{5}{|c|}{ Mechanical Equipment List } & \multicolumn{12}{|c|}{ Scaled Installed costs } \\
\hline EQUIPMENT TITLE & DESCRIPTION & HP & MATERIAL & 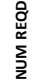 & $\$$ & 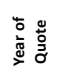 & $\begin{array}{l}\text { Purch cost in } \\
\text { Base } \mathrm{rr}\end{array}$ & Scaling Variable & 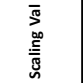 & $\frac{2}{5}$ & 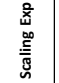 & 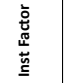 & $\begin{array}{l}\bar{\pi} \\
3_{3}^{3} \\
\frac{3}{2}\end{array}$ & $\begin{array}{l}\stackrel{\circ}{\bar{\pi}} \\
\stackrel{0}{\pi} \\
\tilde{n}\end{array}$ & $\begin{array}{c}\text { Scaled Purch } \\
\text { cost }\end{array} \mid$ & $\begin{array}{c}\text { Purch cost in } \\
\text { Proj year }\end{array}$ & $\begin{array}{l}\text { Inst cost in } \\
\text { Proj year }\end{array}$ \\
\hline Continuous Enzymatic Hydrolysis Bulk & & & & & & & & & & & & & & & & & \\
\hline \begin{tabular}{|l} 
Hydrolyzate Heater \\
Recror
\end{tabular} & Plate \& Frame $32.5 \mathrm{MmBtu} / \mathrm{hr}$ & & 55304 & 1 & $\$ 85,000$ & 2010 & $\begin{array}{r}585,000 \\
50,00\end{array}$ & 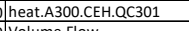 & & Gcal/hr & 0.70 & & (8) & 0.9910 & $\begin{array}{r}84,461 \\
\end{array}$ & $\begin{array}{r}\$ 83,066 \\
50,70\end{array}$ & $\begin{array}{r}\$ 182,745 \\
28,25\end{array}$ \\
\hline Reactor 1 & $\begin{array}{l}\text { Basis }=1,000,000 \text { gallon ea } \\
\text { Basis }=100000\end{array}$ & & 30455 & 1 & $\frac{\$ 844,000}{\$ 84000}$ & 2009 & $\frac{\$ 844,000}{58,000}$ & Volume Flow & 1000000 & gal & 1.00 & & $2,350,067$ & 2.35 & \begin{tabular}{|l}
$\$ 1,983,457$ \\
$\$ 1,05,23$ \\
\end{tabular} & $\begin{array}{l}\$ 2,058,706 \\
\$ 1,07 \\
\end{array}$ & $\begin{array}{l}\$ 3,088,059 \\
2,06199\end{array}$ \\
\hline $\begin{array}{l}\text { Reactor } 2 \\
\text { Reactor } 3 \\
\end{array}$ & \begin{tabular}{|l} 
Basis $1,1,00,0000$ gallon ea \\
Basis $=1,00,000$ gallon ea
\end{tabular} & & $\begin{array}{l}30445 \\
3045 \\
\end{array}$ & $\frac{1}{1}$ & $\begin{array}{l}\$ 844,000 \\
\$ 844,000\end{array}$ & $\frac{2009}{2009}$ & $\begin{array}{l}\$ \$ 84,0,00 \\
\$ 84,4000\end{array}$ & $\begin{array}{l}\text { Volume flow } \\
\text { Volume flow }\end{array}$ & $\begin{array}{l}10000000 \\
100000\end{array}$ & gal & $\frac{1.00}{1.00}$ & $\begin{array}{r}1.5 \\
1.5 \\
\end{array}$ & $\frac{2,257,326}{2,087,882}$ & $\frac{2.26}{2.09}$ & $\begin{array}{l}\$ 1,905,1833 \\
\$ \$, 162,172\end{array}$ & $\begin{array}{l}\$ 1,977,463 \\
\$ 1,289,026 \\
\end{array}$ & $\begin{array}{l}\$ 2,966,194 \\
\$ 2,73,539 \\
\end{array}$ \\
\hline $\begin{array}{l}\text { Keactors } \\
\text { CEHCooler }\end{array}$ & $\begin{array}{l}\text { Pasis } 1,000,000 \text { gallon ea } \\
\text { Plate \& frame }\end{array}$ & & $\frac{3045 s}{3045 s}$ & 1 & 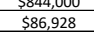 & 2009 & $\begin{array}{r}5844,000 \\
586,928 \\
\end{array}$ & 3 one per reactor & 100000 & ea & $\begin{array}{l}1.00 \\
1.00 \\
\end{array}$ & $\begin{array}{r}1.5 \\
2.2 \\
\end{array}$ & $2,087,882,3$ & 2.25 & $\begin{array}{r}\$, 1,62,1 / 732 \\
\$ 21,732 \\
\end{array}$ & $\$ 1,282,026$ & $\begin{aligned} 5,44,539 \\
49,624 \\
\end{aligned}$ \\
\hline CEH recirculation pump & 340 GPM, 150 FT & 20.0 & 31655 & 1 & $\$ 47,200$ & 2009 & $\$ 47,200$ & one per reactor & & ea & 0.80 & & 3 & 0.25 & $\frac{\$ 2,1504}{\$ 15,570}$ & $\$ 16,161$ & $\$ 37770$ \\
\hline CEH MF membranes & Ultrafiltration- scaled from Nexant value & & & 1 & $\$ 2,048,000$ & 2011 & $\$ 2,048,000$ & area required & 53820 & $f \mathrm{ft}^{2} / \mathrm{ur} \mathrm{r}$ & 1.00 & & 95,783 & 1.78 & $\$ 3,644,804 \mid$ & $\$ 3,370,993$ & $\$ 8,427,482$ \\
\hline CEH UF membranes & Ultrafiltration-scaled from Nexant value & & & 1 & $\$ 2,048,000$ & 2011 & $\$ 2,048,000$ & area required & 53820 & ftr $2 /$ uni & 1.00 & & 114,939 & 2.14 & $\$ 4,373,765$ & $\$ 4,045,191$ & $\$ 10,112,978$ \\
\hline CEH membrane spare & Ultrafiltration-scaled from Nexant value & & & 1 & $\$ 2,048,000$ & 2011 & $\$ 2,048,000$ & units & 53820 & $\sqrt{f t t^{2} / \text { uni }}$ & 1.00 & 2.5 & 31,928 & 0.59 & $\$ 1,214,935$ & $\$ 1,123,664$ & $\$ 2,809,161$ \\
\hline Lignin Press & From EtOH model lignii press + supporting equip & & 31655 & 2 & $\$ 3,294,700$ & 2010 & $\$ 3,294,700$ & strm.A300.CEH.LPRESS.571 & & $\mathrm{kg} / \mathrm{hr}$ & 0.80 & & 22,221 & 0.70 & $\$ 2,472,386$ & $\$ 2,431,538$ & $\$ 4,133,615$ \\
\hline Sugar Concentration & & $3600 \mathrm{~kW}$ & 30455 & 1 & $\$ 6,370,000$ & 2013 & $\$ 6,370,000$ & strm.A300.EVAP.3015UG & 244084 & $\mathrm{~kg} / \mathrm{hr}$ & 0.70 & 2.0 & 788,352 & 3.23 & $\$ 14,473,253$ & $\$ 13,820,133$ & $\$ 27,640,265$ \\
\hline Concentrated Sugar Storage Tank & 5,500 gallons -20 min residence time & $20 \mathrm{hp}$ & ss & 1 & $\$ 168,000$ & 2011 & $\$ 168,000$ & strm.A300.SUG-EVAP & 76712 & $\mathrm{~kg} / \mathrm{hr}$ & 0.70 & 1.8 & 179,172 & \begin{tabular}{l|l}
2.34 & \\
\end{tabular} & $\$ 304,225$ & $\$ 281,370$ & $\$ 506,467$ \\
\hline & & & & & & & & & & & & & & a 300 Totals & $\$ 32,255,943$ & $\$ 31,059,867$ & $\$ 62,697,299$ \\
\hline A400:Enzyme Production & Mechanic & ment List & & & & & & & & Scals, & dinstall & d Costs & & & & & \\
\hline EQUIPMENT TITLE & DESCRIPTION & HP & MATERIAL & 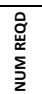 & $\$$ & 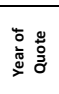 & $\begin{array}{c}\text { Purch cost in } \\
\text { Base } \mathrm{Yr}\end{array}$ & Scaling Variable & 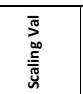 & $\frac{\mathscr{m}}{5}$ & 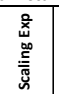 & 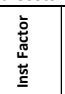 & $\begin{array}{l}\frac{0}{3} \\
\frac{3}{2} \\
\frac{3}{2}\end{array}$ & 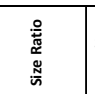 & $\begin{array}{l}\text { Scaled Purch } \\
\text { Cost }\end{array}$ & $\begin{array}{c}\text { Purch Cost in } \\
\text { Proj year }\end{array}$ & $\begin{array}{l}\text { Inst Cost in } \\
\text { Proj year }\end{array}$ \\
\hline Cellulase Fermentor $A$ & & 800.0 & 55316 & & $\$ 580,000$ & 2009 & $\$ 580,000$ & 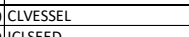 & & ea & 1.00 & 1.5 & & 5.00 & $\$ 2,900,000$ & $\$ 3,010,021$ & $\$ 4,515,032$ \\
\hline $\begin{array}{l}\text { Cellulase Fermentoto Agitators } \\
\text { Cellulase Fermentor Agitators }\end{array}$ & & $\frac{0.75 \mathrm{hp}}{8 \mathrm{hp}}$ & $\frac{55316}{5 \$ 3316}$ & & $\frac{\$ 3,420}{\$ 11,000}$ & 2009 & $\begin{array}{r}\$ 3,420 \\
\$ 11000 \\
\end{array}$ & |ICLSEED & & $\begin{array}{l}\text { ea } \\
\text { ea }\end{array}$ & $\frac{1.00}{100}$ & $\frac{1.5}{1.5}$ & & $\frac{4.00}{4.00}$ & $\begin{array}{l}\$ 13,680 \\
44000\end{array}$ & $\begin{array}{r}\$ 11,499 \\
545,669 \\
\end{array}$ & $\begin{array}{l}\$ 21,298 \\
\$ 68,504 \\
\end{array}$ \\
\hline 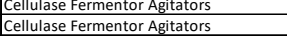 & & $80 \mathrm{hp}$ & $\frac{55310}{55316}$ & & $\begin{array}{l}311,000 \\
\$ 63,000\end{array}$ & 2009 & $\frac{31,0,000}{\$ 63,000}$ & ICLSEED & & ea & $\frac{1.00}{1.00}$ & 1.5 & & $\frac{4.00}{4.00}$ & $\begin{array}{l}\$ \$ 4,000 \\
\$ 252,000 \\
\end{array}$ & $\frac{345,0090}{\$ 261,560}$ & $\begin{array}{l}\$ 30,004 \\
\$ 392,341\end{array}$ \\
\hline 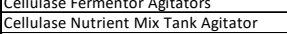 & & $3 \mathrm{hp}$ & $\frac{c s}{c s}$ & 1 & $\$ 4,800$ & 2009 & & strm. 4400.416 & & $\mathrm{~kg} / \mathrm{hr}$ & $\frac{2.50}{0.50}$ & 1.6 & & 70 & $\begin{aligned} 252,000 \\
\$ 4,030\end{aligned}$ & $\frac{321,500}{54,182}$ & $\frac{03.5692}{56,692}$ \\
\hline Cellulase Hold TTank Agitator & & $10 \mathrm{hp}$ & 55316 & 1 & $\$ 26,900$ & 2009 & $\$ 26$ & & 10930 & $\mathrm{~kg} / \mathrm{hr}$ & 0.50 & $\frac{1.5}{1.5}$ & 7,575 & 69 & $\$ 22,395$ & $\$ 23,244$ & $\$ 34,866$ \\
\hline Cellulase Fermentor & $80,000 \mathrm{gal}, 1 \mathrm{~atm}, 28^{\circ} \mathrm{C}$, Internal coil & & sS316 & & $\$ 400,500$ & 2009 & $\$ 400,500$ & CLVESSEL & & ea & 1.00 & 2.0 & & 5.00 & $\$ 2,002,500$ & $\$ 2,078,471$ & $\$ 4,156,943$ \\
\hline 1 1st Cellulase Seed Fermentor & 80 gallon skid complete - $\$ 46,000$ ea & & 30455 & & $\$ 46,000$ & 2009 & & 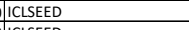 & & ea & 1.00 & 1.8 & & 4.00 & $\begin{array}{l}\$ 184,000 \\
1300\end{array}$ & \begin{tabular}{|l|l|}
$\$ 190,981$ \\
\end{tabular} & $\$ 343,765$ \\
\hline Znd Cellulase Seed Fermentor & complete $\$ 57,500$ ea & & 30455 & & $\$ 57,500$ & 2009 & $\frac{557}{595}$ & IILLEE & & ea & 1.00 & 1.8 & & 4. & $\$ 230,000$ & $\begin{array}{l}\$ 238,726 \\
630,07\end{array}$ & $\$ 429,706$ \\
\hline $\begin{array}{l}\text { 3rd Cellulase Seed Fermentor } \\
\text { Fermentor Air Compressor Package }\end{array}$ & 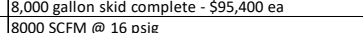 & & $\frac{3045 s}{c 5}$ & 2 & $\begin{array}{l}\$ 95,400 \\
\$ 350000\end{array}$ & 2009 & $\begin{aligned} \$ 95,400 \\
\$ 3500000\end{aligned}-100$ & $\begin{array}{l}\text { ISLSEED } \\
\text { |strm.400.450 }\end{array}$ & & & $\frac{1.00}{0.06}$ & $\frac{1.8}{1.6}$ & 17.8399 & 4.00 & $\$ 381,600 \mid$ & $\begin{array}{l}\mid \$ 396,077 \\
\$ 250395 \\
\end{array}$ & $\begin{array}{l}\$ 712,939 \\
\$ 40,632\end{array}$ \\
\hline $\begin{array}{l}\text { Fermentor Arr compressor package } \\
\text { Cellulase Transfer Pump }\end{array}$ & 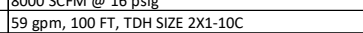 & 3 & 31655 & $\frac{2}{1}$ & $\begin{array}{l}\$ 350,000 \\
\$ 7,357\end{array}$ & 2010 & $\frac{\$ 350,000}{\$ 7,357}$ & strm. .4400 .420 & 133689 & $\mathrm{~kg} / \mathrm{gr} r$ & 0.60 & $\frac{1.6}{2.3}$ & $\mid \begin{array}{cc}\mid 7,859 \\
7,575 \\
\end{array}$ & 0.54 & $\frac{524,242}{54,662}$ & $\begin{array}{r}\$ \$ 4,395 \\
\$ 4,585\end{array}$ & $\begin{array}{l}\$ 40,632 \\
\$ 10,545\end{array}$ \\
\hline Cellulase Seed & TTDH SIZE 2X1-10 & 2 & $3165 \mathrm{~s}$ & 4 & $\$ 29,972$ & 2010 & $\$ 29$, & strm. & & $\mathrm{kg} / \mathrm{hr}$ & 0.80 & 2.3 & 421 & & $\$ 20,408$ & $\$ 20,071$ & 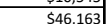 \\
\hline Cellulase Nutrient Transfer Pump & 2 GPM, $100 \mathrm{FT}$ & 1 & 31655 & 1 & 51,500 & 2009 & & & & $\mathrm{~kg} / \mathrm{hr}$ & 0.80 & $\frac{2.3 .}{2.3}$ & 123 & & $\frac{526}{5526}$ & 5546 & $\frac{1.257}{51,257}$ \\
\hline $\begin{array}{l}\text { Cellulase Feed Pump } \\
\end{array}$ & & 1 & 31655 & 1 & $\$ 5,700$ & 2009 & $\$ 5,700$ & & 18168 & $\mathrm{~kg} / \mathrm{hr}$ & 0.80 & 2.3 & 7,575 & & $\$ 2,831$ & $\$ 2,938$ & 56,759 \\
\hline am Pump & 2 GPM, $100 \mathrm{FT}$ & 1 & 31655 & 1 & $\$ 1,500$ & 2009 & $\$ 1,500$ & & & $\mathrm{~kg} / \mathrm{hr}$ & 0.80 & 2.3 & 7 & 0.69 & $\$ 1,115$ & $\$ 1,157$ & $\$ 2,661$ \\
\hline Cellulase Nutrient Mix Tank & HDPE, 8,000 gal & & HDPE & 1 & 59,000 & 2010 & $\$ 9,000$ & strm.a 400.416 & & $\mathrm{~kg} / \mathrm{hr}$ & 0.70 & 3.0 & 123 & 0.55 & $\$ 5,903$ & $\$ 5,806$ & $\begin{array}{ll}\$ 17,417 \\
\end{array}$ \\
\hline Cellulase Hold Tank & $80,000 \mathrm{gal}$ & & $3045 s$ & 1 & $\$ 248,070$ & 2009 & $\$ 248,070$ & strm.a400.422 & 10930 & $\mathrm{~kg} / \mathrm{hr}$ & 0.70 & 1.8 & 7,575 & 0.69 & $\$ 191,921$ & $\$ 199,202$ & $\$ 358,564$ \\
\hline & & & & & & & & & & & & & & a 400 Totals & $\$ 6,502,813$ & $\$ 6,747,832$ & $\$ 11,526,084$ \\
\hline
\end{tabular}




\begin{tabular}{|c|c|c|c|c|c|c|c|c|c|c|c|c|c|c|c|c|c|}
\hline \multirow{2}{*}{\begin{tabular}{|c|} 
A500: Bioconversion \& Upgrading \\
EQUIPMENT TITLE
\end{tabular}} & \multicolumn{5}{|c|}{ Mechanical Equipment List } & \multicolumn{12}{|c|}{ Scaled Installed Costs } \\
\hline & DESCRPPTION & HP & MATERIAL & 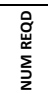 & $\$$ & 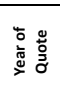 & $\begin{array}{c}\text { Purch Cost in } \\
\text { Base Yr }\end{array}$ & Scaling Variable & 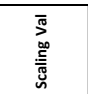 & 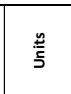 & 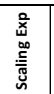 & 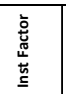 & $\begin{array}{l}\frac{\bar{m}}{3} \\
\text { ב्र } \\
\frac{3}{2}\end{array}$ & 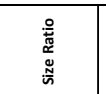 & $\begin{array}{c}\text { Scaled Purch } \\
\text { Cost }\end{array}$ & $\begin{array}{c}\text { Purch cost in } \\
\text { Proj vear }\end{array}$ & $\begin{array}{l}\text { Inst cost in } \\
\text { Proj year }\end{array}$ \\
\hline C4 Acids Anaerobic Fermentation & & & & & & & & & & & & & & & & & \\
\hline \begin{tabular}{|l|} 
Fermentor Feed Cooler \\
Seed Hold Tank Agitator \\
\end{tabular} & \begin{tabular}{|l} 
Plate \& frame \\
\end{tabular} & & $\begin{array}{lll}55304 \\
5304\end{array}$ & $\frac{1}{1}$ & $\frac{\$ 23,900}{\$ \$ 11800}$ & $\frac{2009}{2009}$ & $\frac{\$ 23,900}{\$ 33800}$ & 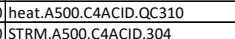 & $\frac{5}{40414}$ & $\frac{6 c a l / / h r}{\mathrm{~kg} g \mathrm{hr}}$ & 0.70 & & & 0.50 & $\begin{array}{ll}\$ \$ 14,638 \\
\end{array}$ & $\$ 15,194$ & $\frac{527,349}{551701}$ \\
\hline $\begin{array}{l}\text { Seed Hold Tank Agitator } \\
\text { 4hth Seed Vessel Aigatotor }\end{array}$ & & $\frac{15 \mathrm{hp}}{7.5 \mathrm{hp}}$ & $\frac{55304}{55}$ & $\frac{1}{2}$ & $\frac{531,800}{\$ 26,000}$ & $\frac{2009}{2009}$ & $\begin{aligned} \\
\$ \$ 13,8000 \\
\$ 26,000\end{aligned}$ & 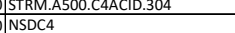 & & $\frac{\mathrm{llg} / \mathrm{hr}}{\text { ea }}$ & 0.50 & $\frac{1.5}{1.5}$ & $\frac{15,918}{2}$ & $\frac{0.39}{1.00}$ & $\frac{\$ 19,957}{\$ 26,000}$ & $\frac{\$ 20,714}{\$ 26986}$ & $\frac{531,071}{54080}$ \\
\hline 5th Seed Vessel Agitator & & $\frac{1.5 \mathrm{mp}}{10 \mathrm{hp}}$ & $\frac{35}{5 s}$ & $\frac{2}{2}$ & $\begin{array}{l}\$ 22,000 \\
\$ 43,000 \\
\end{array}$ & 2009 & $\begin{array}{l}\$ \$ 20,000 \\
\$ 43,000\end{array}$ & NSDC4 & & ea & 0.50 & 1.5. & & $\frac{1.00}{1.00}$ & $\begin{array}{ll}S 2,0,000 \\
\$ 43,000\end{array}$ & $\begin{array}{c}32,9604 \\
\$ 44,631 \\
\end{array}$ & $\begin{array}{ll}\$ 34,480 \\
\$ 66,947\end{array}$ \\
\hline Beer Surge Tank Agitator & & $20 \mathrm{hp}$ & SS304 & 2 & $\$ 68,300$ & 2009 & $\$ 68,300$ & strm.PRD-500 & 425878 & $\mathrm{~kg} / \mathrm{hr}$ & 0.50 & 1.5 & \begin{tabular}{c|c|c|}
11,465 \\
\end{tabular} & 0.03 & $\$ 11,207$ & $\$ 11,632$ & $\$ 17,448$ \\
\hline 1st Seed Fermentor & 20 gallon skid complete - $\$ 37,700$ ea & & $3045 s$ & 2 & $\$ 75,400$ & 2009 & $\$ 75,400$ & & & & 0.70 & & & 1.00 & $\$ 75,400$ & $\$ 78,261$ & $\$ 140,869$ \\
\hline $\begin{array}{l}2 \text { 2nd Seed Fermentor } \\
\text { 2dd Sepd Frmentor }\end{array}$ & 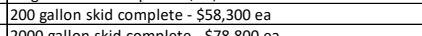 & & 30455 & 2 & $\$ 116,600$ & 2009 & $\begin{array}{l}\$ 116,600 \\
\$ 16760\end{array}$ & NSDC4 & & ea & 0.70 & 1.8 & & 1.00 & $\$ 116,600$ & $\frac{5121,024}{51,570}$ & $\$ 217,842$ \\
\hline $\begin{array}{l}\text { 3rd Seed Fermentor } \\
\text { 4th Seed Fermentor }\end{array}$ & 2000 gallon skid complete $-578,800$ ea & & $\begin{array}{l}30455 \\
30455 \\
\end{array}$ & $\frac{2}{2}$ & $\begin{array}{l}\$ 157,600 \\
\$ 352000\end{array}$ & 2009 & $\begin{array}{r}\$ 157,600 \\
\$ 352000\end{array}$ & NSDC4 & & ea & 0.70 & 1.8 & & 1.00 & $\begin{array}{l}\$ 157,600 \mid \\
352000\end{array}$ & $\begin{array}{c}\$ 163,579 \\
535251\end{array}$ & $\$ 294,442$ \\
\hline 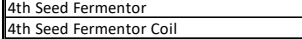 & $\begin{array}{l}2,0,000 \text { agllon, incl. coil - } \$ 176,000 \text { ea } \\
\text { incl. w/tank }\end{array}$ & & $\frac{30455}{30455}$ & $\frac{2}{1}$ & $\frac{\$ 332,000}{\text { INCLUDED }}$ & 2009 & $\$ 352,000$ & INSDC4 & & ea & 0.70 & 2.0 & & 1.00 & & & $\$ 730,709$ \\
\hline 5 5th Seed Fermentor & 200,000 gallon, incl. coil - $\$ 590,000$ ea & & 30455 & 2 & $\$ 1,180,000$ & 2009 & $\$ 1,180,000$ & NSDC4 & & ea & 0.70 & 2.0 & & 1.00 & $\$ 1,180,000$ & $\$ 1,224,767$ & $\$ 2,449,534$ \\
\hline & & & 30455 & 1 & & & & & & & & & & & & & \\
\hline Seed Hold Transfer Pump & $190 \mathrm{GPM}, 150 \mathrm{FT} \mathrm{TDH}$ & 10 & 31655 & & $\$ 8,200$ & 2009 & $\$ 8,200$ & STRM.A500.C4ACID.304 & 43149 & $\mathrm{~kg} / \mathrm{hr}$ & 0.80 & & 15,918 & 0.37 & 53.693 & 53.833 & \\
\hline Seed Hold Tank & 300,000 gallon & & 31655 & $\frac{1}{1}$ & $\frac{0.1000}{\$ 439,000}$ & 2009 & $\$ 439,000$ & STRM.A500.C4ACID.304 & 40414 & $\mathrm{~kg} / \mathrm{hr}$ & 0.70 & 1.8 & $\begin{array}{ll}15,910 \\
15,918\end{array}$ & 0.39 & $\$ 228,668$ & $\$ 237,343$ & $\frac{3,0,010}{\$ 427,217}$ \\
\hline Seed Transfer Pump & 190 GPM, $615 \mathrm{FT} \mathrm{TDH}$ & 40 & $3165 s$ & 2 & $\$ 24,300$ & 2009 & $\$ 24,300$ & STRM.A500.C4ACID.304 & 43149 & $\mathrm{~kg} / \mathrm{hr}$ & 0.80 & 2.3 & 15,918 & 0.37 & $\$ 10,943$ & $\$ 11,358$ & $\$ 26,123$ \\
\hline Fermentor Tank (Anaerobic) & & & 30455 & 12 & $\frac{\$ 10,128,000}{\$ 52,00}$ & 2009 & 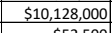 & NVESC4 & & & 1.00 & 1.5 & & 0.25 & $\$ 2,532,000$ & $\$ 2,628,060$ & $\$ 3,942,090$ \\
\hline Fermentor Agitator & & $30 \mathrm{hp}$ & $\frac{55304}{3045}$ & $\frac{1}{12}$ & $\begin{array}{l}\$ 52,500 \\
\$ 86928 \\
\end{array}$ & 2009 & $\begin{array}{l}\$ 52,500 \\
\$ \$ 6928 \\
\end{array}$ & \begin{tabular}{|l|l|} 
NVESCA \\
SNNESC4
\end{tabular} & & ea & $\begin{array}{c}1.00 \\
1.00 \\
\end{array}$ & $\begin{array}{r}1.5 \\
2.2 \\
\end{array}$ & & 3.00 & $\begin{array}{l}\$ 157,500 \\
\$ 21732\end{array}$ & $\begin{array}{l}\$ 163,475 \\
\$ 2256\end{array}$ & $\begin{array}{ll}\$ 245,213 \\
\$ 49,624\end{array}$ \\
\hline $\begin{array}{l}\text { Fermentation Coooler } \\
\text { Fermentation Recirc/Transfer Pump }\end{array}$ & 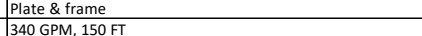 & 20 & $\begin{array}{l}30455 \\
31555 \\
\end{array}$ & $\frac{12}{5}$ & $\begin{array}{r}\$ 86,928 \\
\$ 477200\end{array}$ & $\frac{2009}{2009}$ & $\begin{aligned} \$ 86,928 \\
54,7200\end{aligned}$ & SNEES4 & & ea & $\frac{1.00}{0.80}$ & $\frac{2.2}{2.3}$ & & 0.25 & $\begin{array}{l}\$ 21,732 \\
\$ 15,570\end{array}$ & $\frac{\$ 22,556}{\$ 16,161}$ & $\begin{array}{r}\$ 49,624 \\
\$ 37770 \\
\end{array}$ \\
\hline & $340 \mathrm{GPM}, 150 \mathrm{HI}$ & 20 & $3165 \mathrm{~s}$ & & & $\frac{2009}{2013}$ & $\begin{array}{r}\$ 47,200 \\
9975,000\end{array}$ & stresC4.A500.CAACID.FERM-VNT & $\begin{array}{r}12 \\
13528 \\
\end{array}$ & $\mathrm{sea} / \mathrm{kg}$ & $\frac{0.80}{0.60}$ & & & $\frac{0.25}{1.70}$ & $\begin{array}{l}\$ 1,342,504 \\
\$ 1,304\end{array}$ & $\begin{array}{r}\$ 1,28,01618 \\
\$ 1\end{array}$ & $\frac{\$ 37,1,70}{\$ 2,435,834}$ \\
\hline $\begin{array}{l}\text { BAf Fermentation vent } \\
\text { Bioreactor Transfer Pump }\end{array}$ & 2152 GPM, 171 FT TDH & 125 & 31655 & 1 & $\$ 26,800$ & $\frac{2013}{2009}$ & $\begin{array}{r}\$ 975,000 \\
\$ 26,800\end{array}$ & 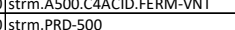 & $\frac{13528}{488719}$ & $\mathrm{~kg} / \mathrm{ghr}$ & $\frac{0.60}{0.80}$ & & $\frac{23,057}{11645}$ & $\frac{1.70}{0.02}$ & $\frac{\$ 1,342,604}{\$ 132}$ & $\frac{\$ 1,282,018}{\$ 1382}$ & $\frac{\$ 2,435,834}{\$ 3379}$ \\
\hline Bioreactor Storage Tank & $1,200,000$ gallon & & 31655 & $\frac{1}{1}$ & $\$ 1,317,325$ & 2011 & $\$ 1,317,325$ & Strm.PRD-500 & 156789 & $\mathrm{~kg} / \mathrm{hr}$ & 0.70 & 2.8 & $\begin{array}{ll}11,405 \\
11,465 \\
\end{array}$ & 0.07 & $\$ 211,128$ & $\begin{array}{l}\$ 1,1382 \\
\$ 195,268 \\
\end{array}$ & $\begin{aligned} \$ \$, 1199 \\
351,482 \\
\end{aligned}$ \\
\hline C4 Recovery and Upgrading & & & & & $31,31,1,25$ & 2011 & $31,217,225$ & & & (I) & 0.10 & & & & 321,120 & S190,200 & \\
\hline Feed Pump & & & & 2 & $\$ 9,200$ & 2009 & $\$ 9,200$ & STRM.A500.C4ACID.AQIN & 22681 & $\mathrm{~kg} / \mathrm{hr}$ & 0.80 & 3.1 & 157,613 & 6.95 & $\$ 43,384$ & $\$ 45,030$ & $\$ 139,593$ \\
\hline Separations (LPME) & & & & 1 & $\$ 8,000,000$ & 2010 & $\$ 8,000,000$ & STRM.A500.CAACID.6 & 362200 & $\mathrm{~kg} / \mathrm{hr}$ & 0.70 & & 173,644 & 0.48 & $\$ 4,781,768$ & $\$ 4,702,766$ & $\$ 9,405,532$ \\
\hline Extraction Economizer & IF calculated in HP column & 654,100 & & & $\$ 376,500$ & 2015 & $\$ 376,500$ & HEAT.A500.CAACID.QX-ORG1 & 2.48 & Gcal/hr & 1.00 & $\begin{array}{ll}1.7 \\
\end{array}$ & & 1.00 & $\begin{array}{l}376,492 \\
\end{array}$ & $\$ 366,282$ & $\$ 6636,348$ \\
\hline \begin{tabular}{|l|} 
Extraction Vacuum Tower \\
Condenso con
\end{tabular} & 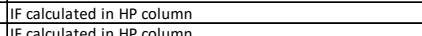 & 924,000 & & & $\frac{\$ 353,700}{\$ 1,000}$ & 2015 & $\begin{array}{l}5353,700 \\
\$ 1100 \\
\end{array}$ & STRM.A500.CAACID.FD-FORG & 38594.00 & kg/hr & $\begin{array}{c}1.00 \\
.01\end{array}$ & 2.6 & 37,107 & 0.96 & $\frac{5340,071}{\$ 10,151}$ & $\begin{array}{ll}330,849 \\
510167\end{array}$ & $\frac{\$ 864,304}{55726}$ \\
\hline \begin{tabular}{|l|} 
Condensor \\
Condensor accumulator
\end{tabular} & 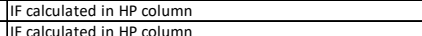 & 62,000 & & & $\frac{\$ 11,000}{\$ 1700}$ & 2015 & $\begin{array}{l}511,000 \\
\$ 1700 \\
17\end{array}$ & HEAT.A500.CAACID.QC.FFRG & $\begin{array}{l}1.31 \\
.31 \\
\end{array}$ & 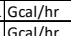 & 1.00 & & & 0.95 & \begin{tabular}{|l|l|l|l}
$\$ 10,451$ \\
$\$ 16246$
\end{tabular} & $\begin{array}{l}\$ 10,167 \\
\$ 158055 \\
\end{array}$ & $\begin{aligned} \$ 57,306 \\
\$ 10092 \\
\end{aligned}$ \\
\hline \begin{tabular}{|l} 
Condensor accumulator \\
Reboiler
\end{tabular} & 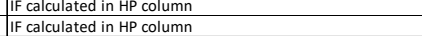 & $\frac{109,2,00}{32,900}$ & - & & $\frac{\$ 17,100}{\$ 217,000}$ & $\frac{2015}{2015}$ & $\begin{aligned} \$ 17,700 \\
\$ 217,000\end{aligned}$ & 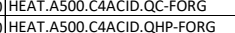 & $\frac{1.31}{-1.51}$ & $\frac{\mid c \mathrm{cal} / / \mathrm{hr}}{\mathrm{Gcal} / \mathrm{hr}}$ & $\frac{1.00}{1.00}$ & 6.4 & & $\frac{0.95}{100}$ & $\begin{aligned} \$ \$ 16,246 \\
\$ 216,43\end{aligned}$ & $\frac{\$ 15,805}{\$ 205}$ & $\frac{\$ 100,932}{\$ 320086}$ \\
\hline $\begin{array}{l}\text { Nefollux pump } \\
\text { Ref }\end{array}$ & IF calculated in $H P$ column & $\begin{array}{ll}322,900 \\
39,100\end{array}$ & & & 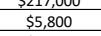 & 2015 & $\frac{\$ 21,000}{\$ 5,800}$ & HEAT.A500.CAACID.QCC-FORG & $\frac{1.51}{1.31}$ & $\frac{G a l / h r}{G c a l / h r}$ & 1.00 & $\frac{1.5}{6.7}$ & $\frac{121}{1}$ & $\begin{array}{l}1.00 \\
0.95 \\
\end{array}$ & $\frac{\$ 216,413}{\$ 5,510}$ & $\frac{5210,544}{55,361}$ & $\begin{array}{l}\$ 320,080 \\
\$ 36,140 \\
\end{array}$ \\
\hline \begin{tabular}{|l|} 
RXR1 Feed Pump \\
DX1 Rec but
\end{tabular} & & & & 2 & $\$ 9,200$ & 2009 & $\begin{array}{l}53,2000 \\
59,200\end{array}$ & STRM.A500.CAACID.AQIN & 22681 & $\mathrm{~kg} / \mathrm{hr}$ & 0.80 & & 157,613 & 6.95 & $\$ 43,384$ & $\$ 45,030$ & $\$ 139,593$ \\
\hline RXR1 preheat & & & SS304 & 1 & $\$ 41,000$ & 2009 & $\$ 41,000$ & HEAT.A500.C4ACID.32 & & Gcal/hr & 0.70 & 2.2 .250 & & 0.27 & $\mid \$ 16,598$ & $|\$ 17,228|$ & $\frac{\$ 15,, 595}{\$ 37,901}$ \\
\hline RXR 1 (Ketonization) & & & & 1 & $\$ 2,044,000$ & 2014 & $\$ 2,044,000$ & STRM.A500.CAACID.16 & 53204 & $\mathrm{~kg} / \mathrm{hr}$ & 0.37 & 2.2 & 21,075 & 0.40 & $\$ 1,451,036$ & $\$ 1,364,392$ & $\$ 3,001,662$ \\
\hline RXR1 Economizer & IF calculated in HP column & 447,300 & & & $\$ 251,000$ & 2015 & $\$ 251,000$ & HEAT.A500.CAACID.QX-KETO & 1.75 & $\mathrm{Gcal} / \mathrm{hr}$ & & 1.8 & & 0.76 & $\$ 251,000$ & $\$ 244,193$ & 5435,170 \\
\hline \begin{tabular}{|l|} 
RXR condensor \\
RXy Conderor
\end{tabular} & & & & 1 & $\frac{\$ 487,000}{50,020}$ & 2010 & $\begin{array}{l}5487,000 \\
56000 \\
\end{array}$ & HEAT.A500.CAACID.31 & 23 & $\frac{6 \mathrm{cal} / / \mathrm{hr}}{\mathrm{col} / \mathrm{s}}$ & 0.60 & 2.8 & & 0.08 & $\frac{5108,327}{\$ 5,287}$ & $\begin{array}{l}106,537 \\
5,381 \\
\end{array}$ & $\frac{\$ 298,303}{661950}$ \\
\hline $\begin{array}{l}\text { RXR1 Condensor } \\
\text { RX12 Phase Flash }\end{array}$ & 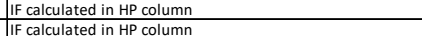 & $\frac{112,700}{12700}$ & 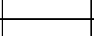 & & $\frac{\$ 26,200}{\$ 22500}$ & $\frac{2015}{2015}$ & $\begin{array}{l}\$ 26,200 \\
\$ 22500\end{array}$ & JEAT.A500.CAACDD.31 & $\begin{array}{r}3.36 \\
22563 \\
\end{array}$ & $\frac{G c a l / h r}{3 k g h r}$ & $\frac{1.00}{100}$ & $\frac{4.3}{5.7}$ & $\frac{2}{21,075}$ & $\frac{0.56}{0.93}$ & $\frac{\$ 14,782}{\$ \$ 2,017}$ & $\frac{\$ 14,381}{\$ \$ 20,471}$ & $\frac{\$ \$ 1,859}{\$ 116046}$ \\
\hline $\begin{array}{l}\text { RXX12 Phase Flash } \\
\text { RXR1 Vent Scrubber }\end{array}$ & 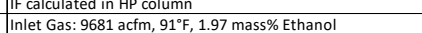 & 127,700 & SS304:pP & & $\$ 22,500$ & $\frac{2015}{2009}$ & \begin{tabular}{r|}
$\$ 22,500$ \\
$\$ 215,000$
\end{tabular} & 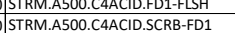 & $\begin{aligned} 222563.00 \\
2208\end{aligned}$ & $\mathrm{kgg/hr}$ & $\frac{1.00}{0.00}$ & & $21,075 \mid$ & 0.93 & $\frac{\$ 21,017}{\$ 129431}$ & $\frac{\$ 20,447}{\$ 134,327}$ & $\frac{\$ 116,046}{\$ 322420}$ \\
\hline 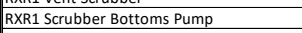 & 108 GPM, 104 FT TDH & & 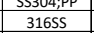 & 1 & $\frac{343,000}{56,300}$ & 2009 & $\begin{aligned} \$ \$ 1,5000 \\
\$ 6,300 \\
\end{aligned}$ & STRM.ASO0.CAAACID.SCRB-BTM & 226587.00 & $\mathrm{~kg} / \mathrm{hr} / \mathrm{hr}$ & 0.80 & 2.3 & & $\frac{0.43}{5.95}$ & $\frac{5199,434}{\$ 26,239}$ & $\frac{\$ 144,344}{\$ 277,234}$ & $\begin{array}{l}\frac{\$ 322,240}{\$ 62,638} \\
\end{array}$ \\
\hline RXR1 Decanter & (4) 9,841 gallon vessels & & 30455 & 4 & $\$ 588,000$ & 2013 & & STRM.ASO0.CAAACID.FDD-S1 & 156789 & $\mathrm{~kg} / \mathrm{hr}$ & 0.50 & & 145,930 & 0.95 & $\begin{array}{ccc}\$ 56,239 \\
\$ 567272\end{array}$ & $\$ 541,673$ & $\$ 1,083,346$ \\
\hline $\begin{array}{l}\text { RXR2 Feed Pump } \\
\end{array}$ & IF calculated in HP column & 124,400 & & & $\$ 52,200$ & & $\frac{153,200}{552,200}$ & STRM.A500.CAACID.FD-RMIX & 237365 & 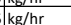 & 1.00 & & 101,236 & 0.43 & $\frac{522,263}{5236}$ & $\frac{1521,660}{5120}$ & $\frac{512031,618}{551,618}$ \\
\hline RXR2 preheat & IF calculated in HP column & 301,500 & & & $\$ 142,600$ & 2015 & $\$ 142,600$ & HEAT.A500.CAACID.OH-RCOND & -5.86 & Gcal/hr & 1.00 & 2.1 & $\frac{(1)}{(1)}$ & 0.14 & $\$ 19,544$ & $\$ 19,014$ & $\$ 40,200$ \\
\hline CSTR ketone condensation (5hr residence & UIF calculated in HP column & $3,601,400$ & & & $\$ 1,993,000$ & 2015 & $\$ 1,993,000$ & STRM.A500.CAACID.22 & 237365.42 & $\mathrm{~kg} / \mathrm{hr}$ & 1.00 & & 303,709 & 1.28 & $\$ 2,550,041$ & $\$ 2,480,885$ & $\$ 4,483,021$ \\
\hline Residence time scaling (redo in ACCE even & titually) & & & & SIZING & & & Residence time & & & & & 3 & 0.20 & & & \\
\hline \begin{tabular}{|l|l|l|} 
CSTR Agitator \\
RXP2HHS
\end{tabular} & from anaerobic fermentor quote & $30 \mathrm{hp}$ & 55304 & 1 & $\begin{array}{lll}\$ 52,500 \\
5500\end{array}$ & 2009 & $\begin{array}{lll}\$ 52,500 \\
58500\end{array}$ & \begin{tabular}{|l} 
STRM.A500.CAACID.22 \\
STPMA50.AACD.11
\end{tabular} & 237365.42 & & 1.00 & $\frac{1.5}{3.5}$ & 101,236 & 0.43 & $\frac{\$ 22,391}{52,591}$ & \begin{tabular}{|lll}
$\$ 23,241$ \\
52601
\end{tabular} & $\frac{\$ 34,861}{\$ 123190}$ \\
\hline $\begin{array}{l}\text { RXX2 HHPS } \\
\text { RR Catalyst Filter(scroll discharge centrifu }\end{array}$ & $\begin{array}{l}\text { II calculated in } \mathrm{HP} \text { column } \\
\text { IF calculated in } \mathrm{HP} \text { column }\end{array}$ & $\frac{296,900}{1000}$ & & & 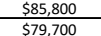 & $\frac{2015}{2015}$ & $\frac{\$ 85,800}{\$ \$ 97,700}$ & 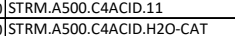 & $\begin{aligned} 237365.42 \\
4464\end{aligned}$ & $\mathrm{~kg} / \mathrm{k} / \mathrm{hr}$ & $\frac{1.00 \mid}{1.00}$ & 3.5.5. & $\frac{101,236}{1,055}$ & $\frac{0.43}{0.24}$ & $\frac{536,594 \mid}{\$ 18,831}$ & $\frac{\$ 35,6011}{\$ 18,320}$ & $\frac{\$ 123,194}{523,148}$ \\
\hline $\begin{array}{ll}\text { Ketone Recycle Tower } \\
\text { Ker d }\end{array}$ & IF calculated in HP column & 943,600 & & & $\$ 286,000$ & 2015 & $\$ 286,000$ & & 232901.00 & & 1.00 & & $\frac{1,053}{100,181}$ & 0.43 & $\begin{array}{lll}\$ 123,0311 \\
\$ 1231\end{array}$ & $\begin{array}{c}\$ 10,3264 \\
\$ 119,684 \\
\end{array}$ & 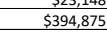 \\
\hline Condensor & If calculated in HP column & 78,200 & & & $\$ 16,400$ & 2015 & $\$ 16,400$ & CID.QC-FRAC & 5.83 & Gcal/hr & 1.00 & 4.8 & 5 & 0.86 & $\$ 14,138$ & $\$ 13,755$ & $\$ 65,588$ \\
\hline Condensor accumulator & in $\mathrm{HP}$ column & 165,200 & & & $\$ 32,600$ & 2015 & $\$ 32,600$ & HEAT.A500.CAACID.QC-FRAC & 5.83 & Gcal/hr & 1.00 & 5.1 & 5 & 0.86 & $\$ 28,104$ & $\$ 27,342$ & $\$ 138,556$ \\
\hline \begin{tabular}{|l|l|} 
Reboiler \\
Reflux pump
\end{tabular} & $\begin{array}{l}\text { IF calculated in } \mathrm{HP} \text { column } \\
\text { III c calculated in } \mathrm{PP} \text { clumn }\end{array}$ & 721,600 & & & $\$ 318,800$ & 2015 & $\begin{array}{r}\$ 318,800 \\
\$ 3100 \\
\end{array}$ & HEAT.A500.CAACD.OHP-FRAC & 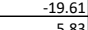 & Gcal/hr & 1.00 & 2.3 .5 & (5) & 0.25 & $\begin{array}{r}\$ 79,525 \\
512203 \\
\end{array}$ & $\$ 77,368 \mid$ & $\frac{\$ 175,122}{\$ \$(1236}$ \\
\hline $\begin{array}{l}\text { Reflux pump } \\
\text { Furnace }\end{array}$ & IF calculated in HP column & 71,700 & & 1 & $\frac{\$ 13,100}{\$ 241,400}$ & $\frac{2015}{2011}$ & $\begin{aligned} \$ 13,100 \\
\$ \$ 21400\end{aligned}$ & 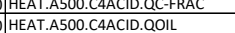 & $\begin{array}{r}5.83 \\
-2.4187 \\
\mathrm{r}\end{array} \mathrm{l} \mathrm{l}$ & $\frac{3 \mathrm{cal} / \mathrm{kr}}{\mathrm{MMcal} / \mathrm{k}}$ & $\frac{1.00}{0.00}$ & $\begin{array}{c}5.5 \\
1.5 \\
\end{array}$ & & $\frac{0.86}{3.30}$ & $\begin{aligned} \$ 11,293 \\
557190\end{aligned}$ & $\frac{\$ 10,987}{\$ 5515,32]}$ & $\frac{\$ 60,136}{\$ 783305}$ \\
\hline $\begin{array}{l}\text { Furnace } \\
\text { C4 DDO }\end{array}$ & & & & 1 & & & & & & & & & & & & & \\
\hline HDO Feed Pump & If calculated in $\mathrm{HP}$ column & 420,200 & & & $\$ 201,600$ & 2015 & $\$ 201,6$ & STTRM & 221115 & $\mathrm{~kg} / \mathrm{hr}$ & 1.00 & 2.1 & 12,137 & & $\$ 11,066$ & $\$ 10,766$ & \\
\hline HDO feed tank & insulated, $6460 \mathrm{gal}$ & & & & 545,966 & 2011 & 545,9 & STRM.A500.CAACID.FAC-BTM & 290932 & $\mathrm{~kg} / \mathrm{hr}$ & 0.60 & $\frac{2.50}{2.50}$ & $\frac{12,137}{12,137}$ & 0.04 & $\$ 1,0,833$ & $\frac{51,100}{\$ 6,320}$ & $\frac{32,440}{515,800}$ \\
\hline HDO reactor pump & & & & & $\$ 802,861$ & 2014 & $\$ 802,861$ & STRM.A500.CAACID.FAC-BTM & 208720 & $\mathrm{~kg} / \mathrm{hr}$ & 0.80 & 1.40 & 12,137 & 0.06 & $\$ 82,469$ & $\$ 77,544$ & $\$ 108,562$ \\
\hline HDO trim preheater & & & 30455 & & $\$ 41,000$ & 2009 & $\$ 41,000$ & HEAT.A500.CAACID.QX-BTM & -2 & MMkcal/ & 0.70 & 2.20 & 1 & 0.34 & $\$ 19,117$ & $\$ 19,842$ & $\$ 43,653$ \\
\hline HDO Fixed Bed Reactor & (Q3 FY17 milestone), base PF=2.5,208 BBL/hr & & & & $\$ 4,168,568$ & 2011 & $\$ 4,168,568$ & Volume Flow (liquid) & 32895 & L/hr & 0.70 & 2.00 & 13,258 & 0.40 & $\$ 2,206,593$ & $\$ 2,040,825$ & $\$ 4,081,651$ \\
\hline \begin{tabular}{|l} 
Pressure Factor (via Guthrie) \\
Internals
\end{tabular} & $(>1000 \mathrm{PSIG}=2.5,900=2.3,800=1.9,700=1.8,600=1.6,500=1.45$ & $100=1.35)$ & 1.6 & & & & & strm.A550.CAACID.HDO-LQ & $\frac{29274}{1}$ & gkg/hr & & & 12,137 & & & & \\
\hline $\begin{array}{l}\text { Hinternals } \\
\text { HDO Effluent econimizer } \\
\end{array}$ & 2-4 TEMA shell and tube $\mathrm{HX}$ & & 31655 & & $\$ 353,600$ & 2011 & $\$ 353,600$ & 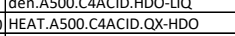 & $\frac{1}{14}$ & 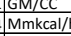 & 0.70 & 2.66 & 3 & 0.19 & $\$ 110,628$ & $\$ 102,317$ & $\$ 271,737$ \\
\hline H2 Makeup Compressor & recipricating compressor(5 stages) & & & & $\$ 1,621,200$ & 2011 & $\$ 1,621,200$ & strm.A50 & & $\mathrm{kg} / \mathrm{hr}$ & 0.60 & 1.09 & 412 & 1.06 & $\$ 1,675,703$ & $\$ 1,549,818$ & $\$ 1,689,103$ \\
\hline H2 Makeup Compressor spare & mpressor(5 stages) & & & & $\$ 1,621,200$ & 2011 & $\$ 1,621,200$ & strm.A50 & & & 0.60 & & & 1.06 & $\$ 1,675,703$ & $\$ 1,549,818$ & $\$ 1,675,719$ \\
\hline H2 Reycle Compressor & ompressor & & & & $\$ 1,103,700$ & 2011 & $\$ 1,103,700$ & $\sqrt{\text { strm.A500.C4AC }}$ & 14665 & $\mathrm{~kg} / \mathrm{kr}$ & 0.60 & 1.13 & 2,434 & 0.17 & 5375,688 & 5347 & \$392,704 \\
\hline H2 Recycle Compressor spare & centrifugal compressor & & & & $\$ 1,103,700$ & 2011 & $\$ 1,103,700$ & strm.A500.CAACID.H2-REC & 14665 & $\frac{\mathrm{kg} / \mathrm{hr}}{\mathrm{h}} \mathrm{s}$ & 0.60 & 1.10 & 2,434 & 0.17 & $\overline{\$ 375}$ & & $\$ 383,543$ \\
\hline HHPS & Via Adipic model(via MB) & & & & $\$ 436,000$ & 2013 & $\$ 436,000$ & strm.A500.CAACID.HHPS-FD & 119841 & $\mathrm{~kg} / \mathrm{hr}$ & 1.00 & 1.50 & $14,983 \mid$ & 0.13 & $\$ 54,509$ & $\$ 52,050$ & $\$ 78,074$ \\
\hline & & & & & & & & & & & & & & rea 500 Totals & $\$ 25,017,926$ & $\$ 24,303,278$ & $\$ 43,799,229$ \\
\hline
\end{tabular}




\begin{tabular}{|c|c|c|c|c|c|c|c|c|c|c|c|c|c|c|c|c|c|}
\hline A600: WWT & Mechanical Equipment List & & & & & & & & & & Install & d Costs & & & & & \\
\hline EQUIPMENT TITLE & DESCRIPTION & нР & MATERIAL & $\begin{array}{l}\text { :̊ } \\
\stackrel{u}{\alpha} \\
\sum_{z}\end{array}$ & $\$$ & 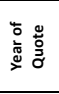 & $\begin{array}{c}\text { Purch cost in } \\
\text { Base } \mathrm{Yr}\end{array}$ & Scaling Variable & 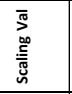 & $\frac{2}{5}$ & 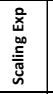 & 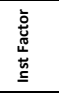 & $\begin{array}{l}\frac{\bar{m}}{3} \\
\frac{3}{2}\end{array}$ & 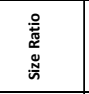 & $\begin{array}{c}\text { Scaled Purch } \\
\text { cost }\end{array} \mid$ & $\begin{array}{c}\text { Purch Cost in } \\
\text { Proj year }\end{array}$ & $\begin{array}{l}\text { Inst cost in } \\
\text { Proj year }\end{array}$ \\
\hline Aeration Basin & Concrete and steel, not installed cost & & Concrete & 3 & $\$ 4,804,854$ & 2012 & $\$ 4,804,854$ & Hydraulic flow & & $M G D$ & 0.60 & 2.1 & & 1.05 & $\$ 4,933,464$ & $\$ 4,571,429$ & $\$ 9,462,858$ \\
\hline Pump - Centrifugal, Aeration Basin Feed & $852 \mathrm{gpm}$ ea & $45 \mathrm{hp}$ & cs & 4 & $\frac{13,30,800}{564,800}$ & 2012 & & & & & & & & & & & \\
\hline Aeration Grid & Full floor aeration grid & & cs & 1 & $\$ 2,500,000$ & 2012 & & & & & & & & & & & \\
\hline $\begin{array}{l}\text { Caustic Feed System } \\
\text { Blowers }\end{array}$ & & $1.5 \mathrm{hp}$ & cs & $\frac{4}{9}$ & $\$ 20,000$ & $\frac{2012}{2012}$ & $\begin{array}{r}\$ 20,000 \\
\$ 207000\end{array}$ & 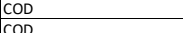 & $\frac{5600}{5600}$ & $\mathrm{~kg} / \mathrm{hr}$ & 0.60 & 3.0 & $\frac{4,149}{4149}$ & 0.74 & $\begin{array}{r}\$ 16,706 \\
1970,05\end{array}$ & $\begin{array}{r}\$ 15,480 \\
10,16\end{array}$ & $\$ 46,440$ \\
\hline $\begin{array}{l}\text { Bowers } \\
\text { Membrane Bioreactor }\end{array}$ & $\begin{array}{l}15000 \text { SCFM @ } 10.3 p s i g \text { ea } \\
\text { Includes membrane, ClP, Scour system }\end{array}$ & $\begin{array}{l}1000 \mathrm{hp} \mathrm{ea} \\
85 \mathrm{hp} \mathrm{ea}\end{array}$ & $\begin{array}{l}\text { cs } \\
c s \\
\end{array}$ & $\begin{array}{l}9 \\
1\end{array}$ & $\$ 2,070,000$ & $\frac{2012}{2012}$ & $\begin{array}{l}\$ 2,070,000 \\
\$ 4,898,500 \\
\end{array}$ & \begin{tabular}{|l|} 
Hydraulic flow \\
\end{tabular} & $\begin{array}{r}5600 \\
2.7 \\
\end{array}$ & $\frac{\mathrm{kg} / \mathrm{h}}{\mathrm{MGD}}$ & 0.00 & 2.0 & $4, \frac{149}{3}$ & $\frac{0.74}{1.05}$ & $\begin{array}{l}\$ 1,7,29,025 \\
\$ 5,118,971 \\
\end{array}$ & $\begin{array}{l}\$ 1,62,1,186 \\
\$ \$, 773,323 \\
\end{array}$ & $\begin{array}{l}\$ 3,204,336 \\
\$ 7,779,050 \\
\end{array}$ \\
\hline Pump, Centrifugal, MBR, RAS & & $160 \mathrm{hp}$ & CS & 6 & $\begin{array}{l}\text { INCLUDED } \\
\text { S75020 } \\
\end{array}$ & 2012 & & & & & & & & & & & \\
\hline $\begin{array}{l}\text { Gravity Belt Thickeners } \\
\text { Contrffoe }\end{array}$ & $2 \mathrm{~m}$ presses & $\begin{array}{ll}48 \mathrm{hp} \\
165 \mathrm{pos}\end{array}$ & cs & 3 & $\begin{array}{l}\$ 750,000 \\
\$ 68800\end{array}$ & 2012 & $\begin{array}{ll}5750,000 \\
566800\end{array}$ & $\frac{C O D}{C O D}$ & 5600 & $\mathrm{~kg} / \mathrm{hr}$ & 0.60 & $\frac{1.6}{2.7}$ & 4,149 & 0.74 & $\begin{array}{ll}5626,468 \\
557368\end{array}$ & $\begin{array}{l}5580,496 \\
\$ 53579\end{array}$ & $\begin{array}{ll}\$ 922,988 \\
\$ 102909\end{array}$ \\
\hline $\begin{array}{l}\text { Centrifuge } \\
\text { Pump, Centrifugal, Centrifuge Feed }\end{array}$ & $105 \mathrm{gpm}$ & $\frac{165 \mathrm{hp} \mathrm{ea}}{15 \mathrm{ph}}$ & $\frac{c s}{c s}$ & $\frac{1}{2}$ & $\frac{\$ 668,800}{\text { INCIUDED }}$ & $\frac{2012}{2012}$ & $\$ 686,800$ & $C O D$ & 5600 & $\mathrm{~kg} / \mathrm{hr}$ & 0.60 & 2.7 & 4,149 & 0.74 & $\$ 573,678$ & $\$ 531,579$ & $\$ 1,429,948$ \\
\hline $\begin{array}{l}\text { Pump, enntrifuga, Centrfigue reed } \\
\text { Pump, Subrsible, Centrate }\end{array}$ & $\frac{105 \mathrm{gpm}}{100 \mathrm{gpm}}$ & $\frac{15 \mathrm{hp}}{10 \mathrm{hp} \text { ea }}$ & $\frac{c s}{c s}$ & $\frac{2}{2}$ & $\frac{\text { IICLCLDED }}{\text { INCLUDED }}$ & $\frac{2012}{2012}$ & & & & & $\rightarrow$ & & & & & & \\
\hline Dewatering Polymer Addition & $9.8 \mathrm{gph}$ neat polymer & 1 hp ea & cs & 2 & INCLUDED & $\frac{2012}{2012}$ & & & & & & & & & & & \\
\hline Conveyor & & $10 \mathrm{hp} \mathrm{ea}$ & $c s$ & 1 & $\$ 7,000$ & $\frac{2012}{2012}$ & $\$ 7,000$ & $C O D$ & 5600 & $\mathrm{~kg} / \mathrm{hr}$ & 0.60 & 2.9 & 4,149 & 0.74 & $\$ 5,847$ & & $\$ 15,495$ \\
\hline Reverse Osmosis & & & & 7 & $\$ 2,450,000$ & $\frac{2012}{2012}$ & $\$ 2,450,000$ & Hydraulic flow & & MGD & $\frac{1.00}{1.00}$ & $\frac{1.8}{1.8}>>3$ & 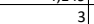 & 1.05 & $\$ 2,560,269$ & $\$ 2,372,388$ & $\begin{array}{l}54,151,679 \\
\end{array}$ \\
\hline Evaporator & $368 \mathrm{gpm}$ & $1480 \mathrm{hp} \mathrm{ea}$ & Titanium & 1 & $\$ 5,000,000$ & 2012 & $\$ 5,000,000$ & Hydraulic flow & & MGD & 0.60 & 1.6 & & 1.05 & $\$ 5,133,833$ & $\$ 4,757,094$ & $\$ 7,658,922$ \\
\hline Ammonia Addition System & $0.63 \mathrm{ggm}$ & $4.5 \mathrm{hp}$ & cs & 4 & $\$ 195,200$ & 2012 & $\$ 195,200$ & $\operatorname{COD}$ & & $\mathrm{kg} / \mathrm{hr}$ & 0.60 & 1.5 & 4,149 & 0.74 & $\$ 163,049$ & $\$ 151,084$ & $\$ 232,669$ \\
\hline Sodium Sulfate Purification & & & & & & & & & & & & & & & & & \\
\hline $\begin{array}{l}\text { Evaporatoto feed tank } \\
\text { Evyanotartor feed heater }\end{array}$ & $\begin{array}{l}\text { linsulated, } 6460 \text { gal } \\
\text { shell and tube } 1 / 1 \text { pass }\end{array}$ & & & & 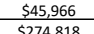 & 2011 & $\frac{\$ 45,966}{\$ 277818}$ & Strm.A600.23 & 290932 & $\mathrm{~kg} / \mathrm{hr}$ & 0.60 & 2.50 & 278,983 & 0.96 & $\begin{aligned} \$ 4,824 \\
\$ 1,5509\end{aligned}$ & 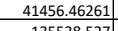 & $\begin{array}{llll}\$ 103,641 \\
\$ 506,616\end{array}$ \\
\hline $\begin{array}{l}\text { Evaporatot reed heater } \\
\text { Evaporator flash drum }\end{array}$ & $\frac{\mid \text { shell and tube } 1 / 2 \text { pass }}{23^{\prime} \times 48^{\prime}-110,000 \text { gal. }}$ & & sS316 & 1 & $\$ 2511,000$ & $\frac{2011}{2009}$ & $\frac{\$ 274,818}{\$ 511,000}$ & 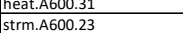 & $\frac{-13}{264116}$ & & $\frac{0.60}{0.70}$ & $\frac{3.00}{200}$ & $\frac{(5)}{278,983}$ & $\frac{0.35}{1.06}$ & $\frac{5146,548}{\$ 530969}$ & $\frac{135538.527}{551113.2787}$ & $\frac{\$ 406,626}{\$ 10226}$ \\
\hline & Nexant quote sodium sulfate, $25410 \mathrm{lb} / \mathrm{hr}$ sc & & 30010 & & $\begin{array}{l}\$ 531,000 \\
\$ 327,680\end{array}$ & $\frac{2099}{2011}$ & $\$ \frac{\$ 511,000}{\$ 327,680}$ & $\begin{array}{l}\text { Strm.A6000.23 } \\
\text { strm.A62SO4 }\end{array}$ & $\frac{204154}{11524}$ & kglim & 0.060 & $\frac{2.00}{23}$ & $\frac{210,305}{1.465}$ & $\frac{1.00}{126}$ & 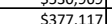 & 551113.12186 & $\frac{\$ 1,102,226}{\$(02209}$ \\
\hline Pryer & Nexant quote Sodium sulfate, $25410 \mathrm{ll} / \mathrm{b} / \mathrm{hr}$ solids basis & & & 1 & $\begin{array}{l}\$ 321,080 \\
\$ 555,008 \\
\end{array}$ & $\frac{2011}{2011}$ & & Strm.A600.PRDD-SALT & & $\mathrm{kg} / \mathrm{hr}$ & 0.60 & $\frac{2.3}{2.6}$ & & 1.20 & $\$ 620,314$ & $\begin{array}{l}\$ 34,8,180 \\
\$ 573,714 \\
\end{array}$ & 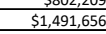 \\
\hline & & & & & & & & & & & & & & 600 Tota & $\$ 22,581,108$ & $\$ 20,981,066$ & $\$ 38,810,731$ \\
\hline
\end{tabular}




\begin{tabular}{|c|c|c|c|c|c|c|c|c|c|c|c|c|c|c|c|c|c|}
\hline \multirow{2}{*}{\begin{tabular}{|c|} 
A700: Lignin Utilization \\
EQUIPMENT TIILE
\end{tabular}} & \multicolumn{5}{|c|}{ Mechanical Equipment List } & \multicolumn{12}{|c|}{ Scaled Installed Costs } \\
\hline & DESCRIPTION & HP & MATERIAL & $\begin{array}{l}\text { ఫ్ } \\
\text { w్ } \\
\sum_{z}^{z} \\
z\end{array}$ & $\$$ & 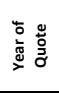 & $\begin{array}{c}\text { Purch Cost in } \\
\text { Base } \mathrm{Yr}\end{array}$ & Scaling Variable & 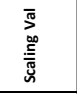 & $\frac{2}{5}$ & 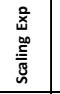 & 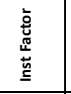 & 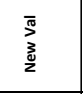 & 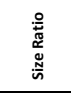 & $\begin{array}{l}\text { Scaled Purch } \\
\text { Cost }\end{array}$ & $\begin{array}{l}\text { Purch cost in } \\
\text { Proj year }\end{array}$ & $\begin{array}{l}\text { Inst cost in } \\
\text { Proj year }\end{array}$ \\
\hline \begin{tabular}{|l|} 
A701: Lignin Conditioning \\
Neutralization Tank
\end{tabular} & & & & & & & & & & & & & & & & & \\
\hline Pulping Reactor Tank & 2.6 atm, $130 \mathrm{C}$ operating (up to $160 \mathrm{c}, 30 \mathrm{~min}$ ) $30 \mathrm{~min}$. hold = & 30,000 gal & $\frac{553316}{55316}$ & 1 & $\$ 16,300,000$ & 2013 & $\$ 16,300,000$ & Strm.A700.A701.PULP-OUT & 323295 & & 0.60 & 2.7 & $\frac{60,0034}{115,134}$ & $\frac{0.36}{0.36}$ & $\$ 8,773,033$ & $\$ \$ \$ 8,377,141$ & $\$ 13,822,282$ \\
\hline \begin{tabular}{|l|l|l|l|l} 
Flash/drain tank \\
\end{tabular} & te & 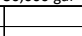 & 55317 & 1 & $\frac{5262,000}{\$ 52600}$ & 2013 & $\frac{51262,000}{\$ 5262,00}$ & strm.A700.A701.PULP-OUT & 323296 & kg/hr & 0.70 & 2.0 & 115,134 & 0.36 & $\frac{5127,181}{\$ 127}$ & $\frac{1311,442}{\$ 121,442}$ & $\$ 242,884$ \\
\hline $\begin{array}{l}\text { Black Liquor Storage Tank } \\
\text { A7720 Muconate Fermentation }\end{array}$ & $1,200,000$ gallon & & 31655 & 1 & $\$ 1,317,325$ & 2011 & $\$ 1,317,325$ & strm.A700.A701.5 & 328984 & $\mathrm{~kg} / \mathrm{hr}$ & 0.70 & 1.8 & 92,912 & 0.28 & $\$ 543,652$ & $\$ 502,811$ & $\$ 905,059$ \\
\hline $\begin{array}{l}\text { A702: Muconate Fermentation } \\
\text { 1st AArobic Seed }\end{array}$ & 80 gallon skid complete - $\$ 46,000$ ea & & & & $\$ 46,000$ & & & & & & & & & & & & \\
\hline 1st Seed Vessel Agitator & 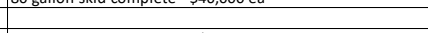 & $0.75 \mathrm{hp}$ & $\frac{30435}{55316}$ & & $\frac{\frac{340,000}{53,420}}{5}$ & 20099 & 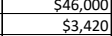 & NSDSD1000 & & ea & $\frac{1.000}{1.00}$ & $\frac{1.80}{1.50}$ & & $\frac{3.00}{3.00}$ & $\frac{\$ 188,000}{\$ 10,260}$ & $\frac{\$ 143,2355}{\$ 10,649}$ & $\frac{\$ 257,824}{\$ 15,974}$ \\
\hline 2nd Aerobic Seed & 800 gallon skid complete $-\$ 57,500$ ea & & 30455 & & $\$ 57,500$ & 2009 & $\$ 57,500$ & NSDD1000 & & ea & 1.00 & 1.80 & & 3.00 & $\$ 172,500$ & $\$ 179,044$ & $\$ 322,280$ \\
\hline 2nd Seed Vessel Agitator & & $8 \mathrm{hp}$ & 55316 & & $\$ 11,000$ & 2009 & $\$ 11,000$ & NSDD1000 & & ea & 1.00 & 1.50 & & 3.00 & $\$ 33,000$ & $\$ 34,252$ & $\$ 51,378$ \\
\hline $\begin{array}{l}\text { Bubble column seed fermentor } \\
\text { Seed cicrulation cooler }\end{array}$ & $\frac{100 \mathrm{~m} 3}{650 \mathrm{sgft}}$ & & 31655 & & $\$ 274,100$ & 2014 & $\begin{array}{rlll}\$ 274,100 \\
5800\end{array}$ & 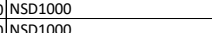 & & $\frac{e a}{\operatorname{san}}$ & 1.00 & 2.30 & & 3.00 & $\frac{\$ 822,300}{\$ 2,200}$ & $\begin{array}{l}\$ 773,199 \\
\$ 2,65\end{array}$ & $\frac{\$ 1,778,358}{\$ 5,323}$ \\
\hline $\begin{array}{l}\text { Seed circulation cooler } \\
\text { Bubble column production fermentor }\end{array}$ & $\frac{6500 \mathrm{sqt}}{1000 \mathrm{~m} 3}$ & - & 31655 & - & $\begin{array}{c}\$ 8,400 \\
\$ \$, 691400\end{array}$ & $\frac{2014}{2014}$ & $\begin{aligned} \$ \$ 8,400 \\
\$ 1691400\end{aligned}$ & $\frac{\text { NSD1000 }}{\text { NEES1000 }}$ & & $\frac{e a}{\text { ea }}$ & 1.00 & $\frac{2.20}{230}$ & & $\frac{3.00}{1700}$ & $\begin{aligned} \$ 25,200 \\
28733,800\end{aligned}$ & 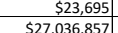 & $\begin{aligned} \$ 52,130 \\
\$ 62184,772 \\
\end{aligned}$ \\
\hline $\begin{array}{l}\text { Dudo } \\
\text { Production circulation cooler }\end{array}$ & $\frac{1000 \mathrm{~m} 3}{4500 \mathrm{saft}}$ & & 31655 & & $\frac{\$ 1,1691,100}{\$ 48,100}$ & $\frac{2014}{2014}$ & $\begin{array}{l}\$ 1,6,61,400 \\
\$ 48,100 \\
\end{array}$ & $\frac{D \text { DVES1000 }}{\text { NVES1000 }}$ & & ea & $\frac{1.000}{1.00}$ & 2.300 & & $\frac{17.00}{17.00}$ & $\begin{aligned} \frac{S 28,773,800}{\$ 817,700} \\
\end{aligned}$ & $\frac{\$ 22,736,8574}{\$ 768,874}$ & 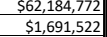 \\
\hline Production circulation pump & $400 \mathrm{gpm}$ & & 31655 & & $\$ 11,500$ & 2014 & $\$ 11,500$ & NVES1000 & & ea & 1.00 & 2.30 & 17 & 17.00 & $\$ 195,500$ & $\$ 183,826$ & $\frac{\frac{\$ 1,099,524}{\$ 422,801}}{5}$ \\
\hline $\begin{array}{l}\text { Fermentation air compressor } \\
\text { Fermentation air receiver }\end{array}$ & 25,000 ACFM @ 45psig; max size in ACCE & & cs & & $\frac{\$ 1,318,600}{\$ 10,600}$ & 2014 & $\frac{\$ 1,318,600}{\$ 104}$ & AlRV1000 & & $m^{\wedge} 3 / s$ & 1.00 & 1.60 & 15 & 1.11 & $\$ 1,464,541$ & $\$ 1,377,090$ & $\$ 2,203,345$ \\
\hline $\begin{array}{l}\text { Fermentation air receiver } \\
\text { Fermentation Surge tank }\end{array}$ & $\mid \frac{25,000 \mathrm{gal}}{\text { insulated cone bottom }, 6460 \mathrm{gal}}$ & & cs & & $\$ 104,600$ & 2014 & $\begin{array}{r}\$ 104,600 \\
\$ \$ 5,966 \\
\end{array}$ & $\begin{array}{l}\mid \text { A AR1 } 1000 \\
\text { |strm.A700.A702.UF-FD }\end{array}$ & 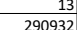 & $\frac{m}{m} 3 / s$ & $\frac{1.00}{0.00}$ & 2.000 & & $\frac{1.11}{0.57}$ & $\begin{aligned} \$ 116,177 \\
\$ \$ 2,656\end{aligned}$ & $\begin{array}{r}\$ 109,240 \\
30203\end{array}$ & $\begin{aligned} \$ 218,480 \\
\$ 75,507\end{aligned}$ \\
\hline $\begin{array}{l}\text { Fermentaction nugge tank } \\
\text { Ultrafiltration membrane separator }\end{array}$ & insulated cone bottom, $6460 \mathrm{gal}$ & & & & $\begin{array}{l}\$ 45,966 \\
\$ 2,048,000\end{array}$ & 2011 & $\begin{aligned} \$ 455,96 \\
\$ 2,048,000 \\
\end{aligned}$ & $\begin{array}{l}\text { Iftsm.A700.A7202.0F-FD } \\
\text { ofolume Flow }\end{array}$ & $\frac{290932}{1303}$ & $\frac{\mathrm{gg} g / \mathrm{hr}}{\mathrm{GPM}}$ & 0.600 & 2.50 & 164,5641 & $\frac{0.57}{1.00}$ & $\begin{array}{r}\$ 32,656 \\
\$ 2,04800\end{array}$ & $\begin{array}{r}\$ 30,203 \\
\$ 1,89,446\end{array}$ & $\begin{aligned} \$ 7,55,57 \\
\$ 4,73,366\end{aligned}$ \\
\hline membrane broth feed pump & & & & & INCLUDED & & $2,0=0,00$ & strm.A700.A702.UF-FD & & $\mathrm{kg} / \mathrm{hr}$ & & & 164,564 & & $\$ 2,048,000$ & $31,094,440$ & $34,130,000$ \\
\hline membrane solvent feed pump & & & & & INCLUDED & & & den.A700.A702.UF-FD & & $\mathrm{gm} / \mathrm{cc}$ & & & & & & & \\
\hline A7003: Recovery and Upgrading & & & & & & & & & & & & & & & & & \\
\hline Carbon Filter & 2 Vessels, for color removal & & & & $\$ 345,234$ & 2011 & $\$ 345,234$ & a Volume flow & & GPM & 0.600 & 2.50 & & 1.00 & $\begin{array}{l}345,234] \\
\end{array}$ & 319298.7157 & $\$ 798,247$ \\
\hline Initial carbon loading & & & & & INCLUDED & 2011 & ( & strm.A700.A703.CFIL-FD & & $\mathrm{kg} / \mathrm{hr}$ & & & 151,994 & & & & \\
\hline & & & & & & & & den.A700.A703.CFIL-FD & & $\mathrm{gm} / \mathrm{cc}$ & & & & & & & \\
\hline CCM Crystallizer & Oslo Type. 2 In series & & 31655 & 2 series & $\$ 7,104,192$ & 2011 & $\$ 7,104,192$ & Volume Flow & 190 & GPM & 0.60 & 2.50 & $\begin{array}{r}39 \\
1164\end{array}$ & 0.21 & $\$ 2,757,128$ & 2550001.932 & $\$ 6,375,005$ \\
\hline & & & & & $\begin{array}{l}\text { INCUUDED } \\
\text { INCLUDED }\end{array}$ & & & $\frac{\mid \text { strm.A700..7703.CRY1-PRD }}{\text { den.A700.A703.CRY1-PRD }}$ & & $\mathrm{kg} / \mathrm{hr}$ & 0.60 & 2.50 & 11,164 & & & & \\
\hline CCM Centrifuge & Centrifuge Separator & & & & $\$ 327,680$ & 2011 & $\$ 327,680$ & strm.A700.A703.CRY1-PRD & 13403 & $\mathrm{~kg} / \mathrm{gcc}$ & 0.60 & 2.30 & 11,164 & 0.83 & 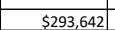 & 271582.9452 & $\$ 624,641$ \\
\hline CCM Drier & Fluidized bed drier parallel & & & 2 parallel & $\$ 555,008$ & 2011 & $\$ 555,008$ & strm.A700.A703.DRY1-PRD & 11526 & $\mathrm{~kg} / \mathrm{hr}$ & 0.60 & 2.60 & 10,613 & 0.92 & $\$ 528,188$ & 488508.7424 & $\$ 1,270,123$ \\
\hline Dissolution Tank & mixing tank to redissolve crystals in solvent (EtOH) & & & & $\frac{\$ 1,317,325}{563000}$ & 2011 & $\$ 1,317,325$ & Strm.A700.A703.FIL2-FD & 328984 & $\mathrm{~kg} / \mathrm{hr}$ & 0.70 & 1.80 & 52,507 & 0.16 & $\$ 364,606$ & 337215.6969 & $\$ 606,988$ \\
\hline $\begin{array}{l}\text { Dissolution Tank agitator } \\
\text { Filtration Centrifuge(salt removal) }\end{array}$ & $\begin{array}{l}\text { pump to retain crystal sulpension } \\
\text { pumoves precipitated soliss after dissolution }\end{array}$ & 80 & $3165 \mathrm{~s}$ & 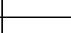 & $\frac{\$ 63,000}{\$ 327,680}$ & $\frac{2009}{2011}$ & $\begin{array}{r}\$ 63,000 \\
\$ 337680 \\
\end{array}$ & Work.A700.A703.W-E-ETOHMX & & $\mathrm{kW}$ & 1.00 & 1.50 & $\frac{60}{105}$ & 1.00 & $\frac{\$ 63,000}{\$ 2061}$ & 65390.10165 & $\$ 98,085$ \\
\hline $\begin{array}{l}\text { Hritration Centrifuge(salt removal) } \\
\text { HDO feed tank }\end{array}$ & $\begin{array}{l}\text { removes precipitated solids atter cilissolution } \\
\text { insulated, } 6460 \text { gal }\end{array}$ & & & & $\$ 347,680$ & 2011 & $\begin{array}{rlll}\$ 322,680 \\
\$ 45,966\end{array}$ & 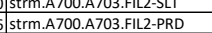 & $\frac{13403}{290932}$ & $\mathrm{~kg} / \mathrm{k} / \mathrm{hr}$ & 0.60 & 2.30 & $\begin{array}{r}14362 \\
52,362\end{array}$ & 0.01 & $\begin{array}{l}5 \$ 1,686 \\
\$ 16,428\end{array}$ & $\begin{array}{ccc}20055.7257 \\
15193.43013\end{array}$ & $\begin{array}{llll}\$ 46,131 \\
\$ 37,984\end{array}$ \\
\hline HDO reactor pump & & & & & $\$ 802,861$ & 2014 & & 1 strm.A700.A703.FIL2-PRD & 208720 & $\mathrm{~kg} / \mathrm{hr}$ & 0.80 & 1.40 & 52,362 & 0.25 & $\begin{array}{cccc}\$ 20,4285 \\
\$ 265.585\end{array}$ & 249726.104 & $\$ 349,617$ \\
\hline HDO Feed Effluent economizer & 2-4 TEMA shell and tube $\mathrm{HX}$ & & 31655 & & $\$ 353,600$ & 2011 & $\$ 353,600$ & heat.A700.A703.QX-HDO & 14 & Mmkall/ & o. 0.0 & 2.66 & (2) & & 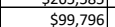 & 92298.9292956 & $\$ 245,130$ \\
\hline HDO trim preheater & & & $3045 s$ & & $\$ \$ 41,000$ & 2009 & $\$ 441,000$ & heat.A700.A703.QH-TRIM & & MMkcal/ $/$ & 0.70 & $\frac{2.20}{2.20}$ & & 0.00 & 50 & 年 & $\begin{array}{llll}50 \\
524,150\end{array}$ \\
\hline HDO Fixed Bed Reactor & (Q3, FF17 milestone), base PF=2.5,208 BBL/hr & & & & $\$ 4,168,568$ & 2011 & $\$ 4,168,568$ & Volume flow (liquid) & 32895 & L/hr & 0.70 & 2.00 & 64,618 & 1.96 & $\$ 6,687,190$ & 6184823.24 & $\$ 12,369,646$ \\
\hline Pressure Factor (via Guthrie) & $(>1000 \mathrm{PSI} G=2.5,900=2.3,800=1.9,700=1.8,600=1.6,500=1.45$, & $400=1.35)$ & 1.6 & & INCLUDED & & & strm.A700.A703.RXR-FD & 29274 & $\mathrm{~kg} / \mathrm{hr}$ & & & 52,362 & & & & \\
\hline Internals & & & & & INCLUDED & & & den.A700.A703.RXR-FD & & $1 \mathrm{GM} / \mathrm{CC}$ & & & & & & & \\
\hline $\begin{array}{l}\text { Hydrogenation Intercoooler (bed1) } \\
\text { Hydrogenation }\end{array}$ & & & & & $\frac{\$ 2,353,181}{\$ 25311}$ & 2007 & $\begin{aligned} \$ 2,353,181 \\
5,35381 \\
\end{aligned}$ & 1 heat.A700.A703.QC-BED1 & $\frac{32}{32}$ & MMkcal/ & 0.65 & 2.21 & & 0.05 & $\$ 349,789$ & 360640.6048 & $\begin{aligned} \$ 797,016 \\
\$ 102678\end{aligned}$ \\
\hline $\begin{array}{l}\text { Hydrogenation Intercooler (bed2) } \\
\text { H2 Makeup Compressor }\end{array}$ & reciprocating compressor(5 stages) & & & & $\frac{\$ 2,353,181}{\$ \$, 122,1200}$ & $\frac{2007}{2011}$ & $\frac{\$ 2,353,181}{\$ 1,621200}$ & 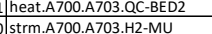 & $\frac{32}{390}$ & MMkcal// & $\frac{0.65}{0.60}$ & $\frac{2.21}{1.09}$ & 33 & $\frac{0.09}{1.02}$ & $\begin{array}{r}\$ 475,158 \\
51,639741\end{array}$ & 489899.38277 151657048 & $\frac{\$ 1,082,678}{\$ 1,1522833}$ \\
\hline $\begin{array}{l}\text { H2 W2 Makeup Comp Compressor } \\
\text { H2 spare }\end{array}$ & 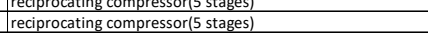 & & & & $\frac{\$ 1,62,1200}{\$ 1,621,200}$ & $\frac{2011}{2011}$ & $\frac{\$ 1,621,200}{\$ 1,621,200}$ & 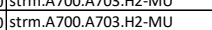 & & 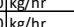 & 0.660 & $\frac{1.099}{1.08}$ & & $\frac{1.02}{102}$ & 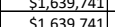 & $\frac{1516577.048}{1516557048}$ & $\begin{array}{ll}\$ 1,652,835 \\
\$ 1,639,756\end{array}$ \\
\hline HHPS & Via Adipic model(via MB) & & & & $\frac{\$ 1,02,1200}{\$ 436,000}$ & 2013 & & strm.A700.A703.HHPS-FD & 119841 & $1 \mathrm{~kg} / \mathrm{hr}$ & 1.00 & 1.50 & 52,759 & 0.44 & $\frac{\$ 1,150,4,41}{\$ \$ 191,946}$ & 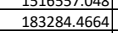 & $\frac{\$ 1,059,150}{\$ 274,927}$ \\
\hline HDO hot gas cooler & & & & & & 2011 & & Dheat.A7 & & IMkcall & $\frac{1.070}{0.70}$ & & & 0.01 & 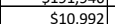 & 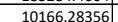 & 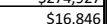 \\
\hline & 3-Phase horizontal sep., demister, $3 / 16$ SS316 cladding & & & & $\$ 328,500$ & $\begin{array}{ll}2011 \\
2011\end{array}$ & $\$ 328,500$ & tive & & 1 L/hr & 0.70 & 2.59 & & 0.00 & 54,857 & 44492.299583 & $\$ 11,623$ \\
\hline PSA - Hydrogenation & & & & & 5975,000 & 2013 & 50 & |strm.A700.A703.CHPS-VAP & 13528 & $3 \mathrm{~kg} / \mathrm{hr}$ & 0.60 & 1.90 & 104 & 0.01 & 50 & 0 & 50 \\
\hline AA evaporator feed tank & linsulated, $6460 \mathrm{gal}$ & & & & 545,9 & 2011 & $\$ 45,966$ & Strm.A700.A703.EVAP-FD & 290932 & $\mathrm{~kg} / \mathrm{hr}$ & 0.60 & 2.50 & 92,707 & 0.32 & $\$ 23,144$ & 21404.92784 & $\$ 53,512$ \\
\hline AA evaporator feed heater & shell and tube $1 / 2$ pass & & & & $\$ 274,818$ & 2011 & $\$ 274,818$ & heat.A700.A703.OH-EVAP & -13 & BMMkcal/ & 0.60 & 3.00 & (2) & 0.12 & $\$ 76,378$ & 70639.95608 & $\$ 211,920$ \\
\hline $\begin{array}{l}\text { AA evaporator flash drum } \\
\text { AA condenser drum }\end{array}$ & $23^{2} \times 48^{\prime}-110,000 \mathrm{gal}$. & & 55316 & 1 & $\frac{\$ 511,000}{\$ 48000}$ & 2009 & $\frac{\$ 511,000}{\$ \$ 877000}$ & Dstrm.A700.A703.EVAP-FD & 264116 & $5 \mathrm{~kg} / \mathrm{hr}$ & 0.70 & 2.00 & 92,707 & 0.35 & $\frac{\$ 245,552}{\$ 26795}$ & 254868.3281 & $\begin{aligned} \$ 509,737 \\
5377010\end{aligned}$ \\
\hline $\begin{array}{l}\text { AA condenser r drum } \\
\text { AA Crystallizer }\end{array}$ & Oslo Type. 2 In series & & $3165 \mathrm{~s}$ & 2 series & $\begin{array}{l}\$ 487,000 \\
\$ 7,104,192\end{array}$ & $\frac{2010}{2011}$ & $\begin{aligned} & \$ 487,000 \\
\$ 7,104,192 & \end{aligned}$ & & & MMkcal/ & $\frac{0.60}{0.600}$ & 2.800 & $\begin{array}{r}9 \\
42 \\
\end{array}$ & $\frac{0.37}{0.22}$ & $\begin{aligned} \$ 267,785 \\
\$ 2857233\end{aligned}$ & $\begin{array}{l}263360.65944 \\
2642595.854\end{array}$ & $\frac{\$ 737,410}{\$ 6,606,490}$ \\
\hline $\begin{array}{l}\text { AA C Crystallizer } \\
\text { AA Centrifuge separator }\end{array}$ & $\begin{array}{l}\text { Usiol Yye.e. In sertes } \\
\text { Centrifuge Separator }\end{array}$ & & 3165s & 2 series & $\frac{\$ \$ l, 104,192}{\$ 327,680}$ & $\frac{2011}{2011}$ & $\frac{\$, \$ 104,192}{\$ 327,680}$ & strm.A700.A703.CRY2-PRD & & $\frac{G G M}{\mathrm{~kg} / \mathrm{hr}}$ & $\frac{0.60}{0.60}$ & $\frac{2.50}{2.30}$ & $\begin{array}{r}42 \\
11,336\end{array}$ & $\frac{0.22}{0.85}$ & $\frac{\$ 52,85,1,43}{\$ 29,355}$ & $\frac{26452599.8544}{274091.7995}$ & $\frac{\$ \$, 606,400}{\$ 630,411}$ \\
\hline AA Drier & Fluidized bed drier parallel & & & 2 parallel & $\$ 555,008$ & 2011 & $\begin{array}{l}\$ 5535,008 \\
\$ 555\end{array}$ & strm.A700.A703.DRY2-PRD & 11526 & $5 \mathrm{~kg} / \mathrm{hr}$ & 0.60 & 2.60 & 10,798 & 0.94 & $\$ 533,697$ & 493603.6734 & $\$ 1,283,370$ \\
\hline & & & & & & & & & & & & & & Totals: & $\$ 64,207,402$ & $\$ 60,344,005$ & $\$ 127,474,183$ \\
\hline
\end{tabular}




\begin{tabular}{|c|c|c|c|c|c|c|c|c|c|c|c|c|c|c|c|c|c|}
\hline A800: CHP & Mechanical Equipment List & & & & & & & & & & Insta & ed Costs & & & & & \\
\hline EQUIPMENT TITLE & DESCRIPTION & HP & MATERIAL & 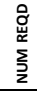 & $\$$ & 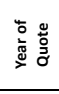 & \begin{tabular}{|} 
Purch Cost in in \\
Base Yr
\end{tabular} & Scaling Variable & 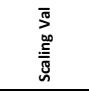 & $\frac{n}{5}$ & 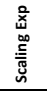 & 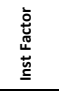 & 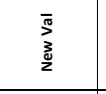 & 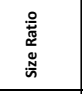 & $\begin{array}{l}\text { Scaled Purch } \\
\text { Cost }\end{array}$ & $\begin{array}{l}\text { Purch cost in } \\
\text { Proj year }\end{array}$ & $\begin{array}{l}\text { Inst Cost in } \\
\text { Proj year }\end{array}$ \\
\hline \begin{tabular}{|l} 
High Solids Burner and Turbine \\
Burner Combustion Air Prenheater
\end{tabular} & & & & & & & & & & & & & & & & & \\
\hline $\begin{array}{l}\text { Burner Combustion Air Preheater } \\
\text { BFW Preheater }\end{array}$ & $\begin{array}{l}\text { INALLUDED } \\
\text { INCLUDED }\end{array}$ & & & $\frac{1}{1}$ & $\begin{array}{l}\frac{\text { INCLUDED }}{\text { INCLUDED }} \\
\end{array}$ & & & & & & & & & & & & \\
\hline Pretreatment/BFW heat recovery & $9.4 \mathrm{MM} \mathrm{Btu} / \mathrm{hr}$ & & 55304 & 1 & $\$ 41,000$ & 2009 & $\$ 41,000$ & oheat.A800.A810.QH812 & & Gcal/hr & 0.70 & $2.2 .250 \mathrm{lal}$ & (2) & 0.63 & $\$ 29,613$ & $\$ 30,737$ & $\$ 67,621$ \\
\hline Air Intake Fan & INCLUDED & & & & INCLUDED & & & & & & & & & & & & \\
\hline $\begin{array}{l}\text { Boiler } \\
\text { Combustion Gas Baghouse }\end{array}$ & 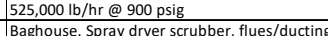 & & cs & $\frac{1}{1}$ & $\frac{\$ 28,550,000}{\$ 1000,000}$ & 2010 & $\$ 28,550,000$ & 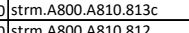 & 238203 & $\frac{k g / h r}{k g}$ & $\frac{0.60}{0.60}$ & 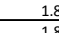 & \begin{tabular}{|c|c|c|}
141,781 \\
126523
\end{tabular} & 0.60 & $\$ 20,912,586$ & $\$ 20,567,081$ & $\$ 37,020,745$ \\
\hline $\begin{array}{l}\text { Combustion Gas aghouse } \\
\text { Turbine//Generator }\end{array}$ & $\begin{array}{l}\text { Baghouse, Spray dryer scrubber, flues/ducting } \\
23.6 \mathrm{~kW}, 2 \text { extractions }\end{array}$ & & & $\frac{1}{1}$ & $\frac{\$ 11,000,000}{\$ 9,500,000}$ & $\frac{2013}{2010}$ & $\begin{array}{rl}5 \mathrm{c} & 59,500,00 \mathrm{c} \\
\end{array}$ & |f tstrm.A800.A810.812 & $\frac{2382203}{-42200}$ & $\mathrm{~kg} / \mathrm{gr}$ & 0.60 & 1.8 & $\frac{126,623]}{(20,318)}$ & 0.53 & $\begin{aligned} 50 \\
56,127,228 \\
\end{aligned}$ & $\begin{aligned} 50 \\
56025.997 \\
\end{aligned}$ & \begin{tabular}{r|r|}
$\$ 0$ \\
51080795 \\
\end{tabular} \\
\hline Hot Process Water Softener System & & & & 1 & 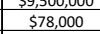 & 2010 & $\begin{aligned} 59,500,000 \\
\$ 78,000 \\
\end{aligned}$ & $\begin{array}{l}\text { ofwork.A900.wtotal } \\
\text { ostrm.A800.A810.812 }\end{array}$ & 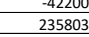 & $\mathrm{kW} / \mathrm{kg} / \mathrm{hr}$ & 0.60 & $\frac{1.8}{1.8}$ & $\frac{(22,318)}{126,623}$ & 0.54 & $\begin{array}{r}\$ 6,127,228 \\
\$ 53,712 \\
\end{array}$ & $\begin{aligned} \$ 6,025,997 \\
\$ \$ 52,825 \\
\end{aligned}$ & $\$ 10,849,795$ \\
\hline Amine Addition Pkg. & & & & 1 & $\$ 40,000$ & 2010 & $\$ 40,000$ & oftrm.A800.A810.812 & 235803 & $\mathrm{~kg} / \mathrm{hr}$ & 0.00 & 1.8 & 126,623 & 0.54 & $\$ 40,000$ & $\$ 39,339$ & $\begin{array}{l}\$ \$ 50,084 \\
70,810 \\
\end{array}$ \\
\hline $\begin{array}{l}\text { Ammonia Addition Pkg } \\
\text { Phoshphate Addition Pg.g. }\end{array}$ & & & & 1 & $\begin{array}{l}\text { INCLLUED } \\
\text { INCLDDED }\end{array}$ & & & & & & & & & & & & \\
\hline $\begin{array}{l}\text { Thosphate Adadtion nKg. } \\
\text { Condensate Pump }\end{array}$ & & & 55316 & $\frac{1}{2}$ & $\begin{array}{l}\text { INCLLDED } \\
\text { INCLUDED }\end{array}$ & & & & & & & & & & & & \\
\hline Turbine Condensate Pump & & & 55304 & 2 & INCLUDED & & & & & & & & & & & & \\
\hline Deaerator Feed Pump & & & 55304 & 2 & INCLUDED & & & & & & & & & & & & \\
\hline BFW Pump & & & 55316 & 5 & INCLUDED & & & & & & & & & & & & \\
\hline Blowdown Pump & & & & & INCLUDED & & & & & & & & & & & & \\
\hline Amine Transfer Pump & & & cs & 1 & INCLUDED & & & & & & & & & & & & \\
\hline Condensate Collection Tank & & & A285C & 1 & INCLUDED & & & & & & & & & & & & \\
\hline $\begin{array}{l}\text { Condensate Surge Drum } \\
\text { Denartor }\end{array}$ & & & 55304 & 1 & INCLUDED & & & & & & & & & & & & \\
\hline Deaerator & Tray type & & Cs;sS316 & 1 & $\$ 305,000$ & 2010 & $\$ 305,000$ & |0|strm.A800.A810.812 & 235803 & $\mathrm{~kg} / \mathrm{hr}$ & 0.60 & 3.0 & $\frac{126,623]}{\operatorname{Arg}}$ & 0.54 & 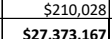 & $\begin{aligned} \$ 206,558 \\
\$ \$ 26.9253\end{aligned}$ & $\begin{aligned} \$ 6619,674 \\
\$ \$ 48,70730\end{aligned}$ \\
\hline A900: Utilities \& Storage & Mecl & nent List & & & & & & & & & $d$ Insta & ed Costs & & & & & \\
\hline EQUIPMENT TITLE & DESCRIPTION & HP & MATERIAL & 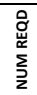 & $\$$ & 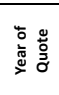 & $\begin{array}{l}\text { Purch cost in } \\
\text { Base } \mathrm{Yr}\end{array}$ & Scaling Variable & 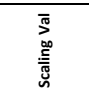 & $\frac{n}{5}$ & 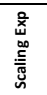 & 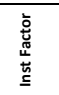 & 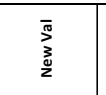 & 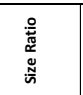 & $\begin{array}{c}\text { Scaled Purch } \\
\text { cost }\end{array}$ & $\begin{array}{l}\text { Purch cost in } \\
\text { Proj year }\end{array}$ & $\begin{array}{l}\text { Inst Cost in } \\
\text { Proj year }\end{array}$ \\
\hline $\begin{array}{l}\text { Utilities System } \\
\text { Cooling Tower System }\end{array}$ & & & & & & & & & & & & & & & & & \\
\hline $\begin{array}{l}\text { Cooling Tower System } \\
\text { Plant Air Compressor }\end{array}$ & $\begin{array}{ll}44,200 \text { gpm } \\
400 \text { sFM@125 psig }\end{array}$ & $\frac{750 \mathrm{hp}}{150 \mathrm{hp}}$ & FiBERGLASS & $\frac{1}{1}$ & $\frac{\$ 1,375,000}{\$ 28,000}$ & $\frac{2010}{2010}$ & $\frac{\$ 1,375,000}{\$ 28,000}$ & $\frac{\text { ostrm.9.900.945 }}{\text { D DRY101 }}$ & 100378300 & & 0.60 & & $\begin{array}{r}16,869,770 \\
33,333\end{array}$ & $\frac{1.68}{1.00}$ & $\begin{array}{l}\$ 1,877,520 \\
\$ 28,000\end{array}$ & $\$ 1,846,501$ & $\$ 2,769,751$ \\
\hline Chilled Water Package & $2 \times 2350$ tons $(14.2 \mathrm{MM} \mathrm{kcal} / \mathrm{hr})$ & $3400 \mathrm{hp}$ & & 1 & $\$ 1,275,750$ & 2010 & $\$ 1,275,750$ & ofheat.a900.qchwop & $\frac{14}{14}$ & Gcal/hr & 0.60 & $\frac{1.6}{1.6}$ & $\begin{array}{r}\frac{30,750}{49} \\
\end{array}$ & 3.47 & $\$ 2,689,031$ & $\frac{52,64,604}{\$ 204}$ & $\begin{array}{l}\$ 44,060 \\
\$ 4,231,366 \\
\end{array}$ \\
\hline CIP System & $100,000 \mathrm{GAL}$ & & SS304/SS31 & 1 & $\$ 421,000$ & 2009 & $\$ 421,000$ & strm.a900.914 & & $\mathrm{kg} / \mathrm{hr}$ & 0.60 & 1.8 & 145 & 2.30 & $\$ 694,222$ & 5720,560 & $\$ 1,297,008$ \\
\hline Cooling Water Pump & 16,120 GPM, 100 FT TDH SIZE $20 \times 20-28$ & 500.0 & cs & 3 & $\$ 283,671$ & 2010 & $\$ 283,671$ & 1 strm.a900.945 & 10982556 & $\mathrm{~kg} / \mathrm{hr}$ & 0.80 & 3.1 & $16,869,770$ & 1.54 & $\$ 399,889$ & $\$ 393,282$ & $\$ 1,219,175$ \\
\hline Make-up Water Pump & 685 GPM, 75 FT TDH SIZE EX4-13 & 20.0 & cs & 1 & $\begin{array}{ll}\$ 6,864 \\
\$ 1,207\end{array}$ & 2010 & $\begin{array}{c}56,864 \\
51,20\end{array}$ & 4 strm.a900.904 & 155564 & $\mathrm{~kg} / \mathrm{hr}$ & 0.80 & 3.1 & $\begin{array}{r}632,929 \\
\end{array}$ & 4.07 & $\begin{array}{l}\$ 21,093 \\
52551 \\
5251\end{array}$ & $\$ 20,744$ & $\begin{array}{l}\$ 64,308 \\
50,201\end{array}$ \\
\hline \begin{tabular}{|l} 
Process Water Circulating Pump \\
Instrument Air Dryer
\end{tabular} & $\frac{2285 \text { GPM, } 75 \text { F T TDS SIIE } 8 X 6-13}{\text { 6670 SCFM - CYCLING TYPE }}$ & 75.0 & cs & $\frac{1}{1}$ & $\frac{\$ 15,292}{\$ \$ 15,000}$ & $\frac{2010}{2009}$ & $\frac{\$ 15,292}{\$ \$ 5,000}$ & $\begin{array}{l}2 \text { strm.a900.905 } \\
\text { DDPY101. }\end{array}$ & $\frac{518924}{83333}$ & $\mathrm{~kg} / \mathrm{hr}$ & 0.80 & $\frac{3.1}{18}$ & $\begin{array}{r}1,334,219 \\
83,33\end{array}$ & $\frac{2.57}{100}$ & $\begin{array}{l}\$ 32,551 \\
\$ \$ 15,000\end{array}$ & $\begin{array}{l}\$ 32,013 \\
\$ 5.569\end{array}$ & $\begin{aligned} \$ 99,241 \\
\$ 28024 \\
\end{aligned}$ \\
\hline $\begin{array}{l}\text { Instrument Air Dryer } \\
\text { Plant Air Receiver }\end{array}$ & 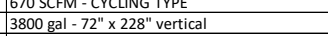 & & cs & $\frac{1}{1}$ & $\begin{array}{l}\$ \$ 15,000 \\
\$ 16,000\end{array}$ & $\begin{array}{l}2009 \\
2009\end{array}$ & $\begin{array}{l}\$ 515,000 \\
\$ 16,000\end{array}$ & DRY101 & 83333 & $\mathrm{~kg} / \mathrm{hr} / \mathrm{hr}$ & 0.60 & $\frac{1.8}{3.1}$ & $\begin{array}{r}8,3333 \\
83,333 \\
\end{array}$ & $\frac{1.00}{1.00}$ & $\begin{array}{l}\$ \$ 15,000 \\
\$ 16,000 \\
\end{array}$ & $\begin{aligned} \frac{515,569}{\$ 16,607} & -1\end{aligned}$ & $\begin{aligned} \$ 25,824 \\
\$ 51,482 \\
\end{aligned}$ \\
\hline Process Water Tank No. 1 & $250,000 \mathrm{gal}$ & & cs & 1 & $\$ 250,000$ & 2009 & $\$ 250,000$ & ostrm.9900.905 & & $\mathrm{kg} / \mathrm{hr}$ & 0.70 & 1.7 & $1,334,219$ & 2.95 & & $\$ 553,953$ & $\begin{aligned} 5941,702 \\
\$ 9420\end{aligned}$ \\
\hline Storage & & & & & & & & & & & & & & & & & \\
\hline Ammonia Storage Tank & $28,000 \mathrm{gal}$ & & SA-516-70 & 2 & & 2010 & $\$ 196,000$ & ostrm.A900.NH3-NET & & $\mathrm{kg} / \mathrm{hr}$ & 0.70 & & 1,359 & 1.16 & $\$ 217,491$ & $\overline{5213,898}$ & $\$ 427,796$ \\
\hline $\begin{array}{l}\text { CSL Storage Tank } \\
\end{array}$ & $70,000 \mathrm{gal}$ & & $\begin{array}{cl}\text { Glass lined } \\
\end{array}$ & 1 & 570,000 & 2009 & $\$ 70,000$ & |strm.A900.CSL-NET & & $\mathrm{kg} / \mathrm{hr}$ & 0.70 & 2.6 & 1,478 & 1.06 & $\$ 72,979$ & 575,748 & $\$ 196,945$ \\
\hline CSL Storage Tank Agitator & & $10 \mathrm{hp}$ & 55304 & 1 & $\$ 21,200$ & 2009 & $\$ 21,200$ & ostrm.A900.CSL-NET & & $\mathrm{kg} / \mathrm{hr}$ & 0.50 & 1.5 & 1,478 & 1.06 & $\$ 21,841$ & $\$ 22,669$ & $\$ 34,004$ \\
\hline $\begin{array}{ll}\text { CSL Pump } \\
\end{array}$ & 8 GPM, $80 \mathrm{FT} \mathrm{TDH}$ & 0.5 & cs & 1 & $\$ 3,000$ & 2009 & $\$ 3,000$ & Dstrm.A900.CSL-NET & 1393 & $\mathrm{~kg} / \mathrm{hr}$ & 0.80 & $3.1 .1-3$ & 1,478 & 1.06 & $\$ 3,146$ & $\$ 3,266$ & $\$ 10,124$ \\
\hline $\begin{array}{l}\text { DAP Bulk Bag Unloader } \\
\text { DPA BUK Kag tolder }\end{array}$ & $\begin{array}{l}\text { Super sack unloader } \\
\text { Sumprackgholder }\end{array}$ & & & 1 & $\begin{array}{l}\$ 30,000 \\
\text { ICILDED }\end{array}$ & 2009 & $\$ 30,000$ & Dstrm.A900.DAP-NET & & $\mathrm{kg} / \mathrm{hr}$ & 0.60 & 1.7 & 714 & 4.38 & $\$ 72,754$ & $\$ 75,515$ & $\$ 128,375$ \\
\hline $\begin{array}{l}\text { DDA Bulk ka g Holder } \\
\text { DAP Make-up Tank }\end{array}$ & $\begin{array}{l}\text { Super rack holder } \\
12,800 \text { gal }\end{array}$ & & 55304 & $\frac{1}{1}$ & $\begin{array}{l}\text { INCLUDED } \\
\$ 102,000\end{array}$ & 2009 & $\$ 102,000 \mathrm{C}$ & 0 strm.A900.DAP-NET & & $\mathrm{kg} / \mathrm{hr}$ & 0.70 & 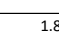 & 714 & 0.44 & 557,580 & $\$ 59,765$ & $\$ 107,576$ \\
\hline DAP Make-up Tank Agitator & & $5.5 \mathrm{hp}$ & 55304 & 1 & $\$ 9,800$ & 2009 & 59,800 & ostrm.A900.DAP-NET & & $\mathrm{kg} / \mathrm{hr}$ & 0.50 & 1.5 & & 4.38 & $\$ 20,504$ & $\$ 21,282$ & $\frac{\$ \$ 10,7516}{\$ 3,1923}$ \\
\hline DAP Pump & 2 GPM, 100 FT TDH & 0.5 & cs & 1 & $\$ 3,000$ & 2009 & $\$ 3,000$ & ostrm.A900 & & $\mathrm{kg} / \mathrm{hr}$ & 0.80 & 3.1 & 714 & 4.38 & 59,775 & 510.146 & $\$ 331.451$ \\
\hline Sulfuric Acid Pump & 5 GPM, 150 FT TDH SIZE $2 X 1-10$ & 0.5 & 55316 & 1 & 57,493 & 2010 & $\$ 7,499$ & & & kg/hr & 0.80 & 2.3 & 10,531 & 5.32 & $\$ 28,518$ & $\$ 28,047$ & $\frac{35.458}{56,508}$ \\
\hline Sulfuric Acid Storage Tank & 2' dia $\times 15^{\prime} \mathrm{H}$ & & ss & 1 & $\$ 96,000$ & $\frac{2010}{2010}$ & $\$ 96,000$ & |strm.A900.ACID-NET & 1981 & $\frac{\mathrm{kg} / \mathrm{hr}}{\mathrm{s}}$ & 0.70 & $1.5 \mathrm{~T}, \mathrm{C}, \mathrm{s}$ & 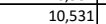 & 5.32 & $\$ 309,155$ & $\$ 304,047$ & 54560071 \\
\hline Caustic Storage Tank & $12,600 \mathrm{gal}, 12^{\prime}$ dia $\times 15^{\prime}$ H & & ss & 1 & $\$ 96,000$ & 2011 & 596,000 & ostrm.A900.BASE-NET & 1981 & $\mathrm{~kg} / \mathrm{hr}$ & 0.70 & 1.5 & 8,235 & 4.16 & $\$ 260,261$ & $\$ 240,709$ & $\$ 361,064$ \\
\hline Firewater Storage Tank & 600,000 gal - 4 hrs @ 2500 gpm & & Glass lined & 1 & $\$ 803,000$ & 2009 & $\$ 803,000$ & ostrm.A900.H2O-FIRE & 8343 & $\mathrm{~kg} / \mathrm{hr}$ & 0.70 & 1.7 & 8,343 & 1.00 & $\$ 803,000$ & $\$ 833,464$ & $\$ 1,416,890$ \\
\hline Firewater Pump & 2500 GPM, 150 FT TDH & 125.0 & cs & 1 & $\$ 15,000$ & 2009 & $\frac{\$ 15,000}{56700}$ & oftrm.A900.H2O-FIRE & 8343 & $\mathrm{~kg} / \mathrm{hr}$ & 0.80 & 3.1 & 8,343 & 1.00 & $\begin{array}{ll}\$ 15,000 \\
<7,0 \leqslant 0\end{array}$ & $\$ 15,569$ & $\$ 48,264$ \\
\hline $\begin{array}{l}\text { Diesel storage tank } \\
\text { Co-Product tstorage Tank(Adipic) }\end{array}$ & 750,000 gal., 7 day storage, Floating roof & & A285C & 1 & $\begin{array}{l}\$ 670,000 \\
690900\end{array}$ & 2009 & $\$ 6770,000$ & $\frac{0 \text { strm.PRD-500 }}{0 \text { tstrm.PRD } 700}$ & $\begin{array}{r}11341 \\
23320025\end{array}$ & kg/hr & 0.70 & $\frac{1.7}{1.80}$ & 11,465 & 1.01 & $\$ 675,160$ & $\$ 5700,774$ & $\begin{aligned} \$ 1,191,317 \\
\$ 798252 \\
\end{aligned}$ \\
\hline $\begin{array}{l}\text { Co-Product tstorage TankkAdidic) } \\
\text { Co-Product Storage Tank (Sodium Sulfate) }\end{array}$ & & & & $\frac{1}{1}$ & $\begin{array}{l}\frac{\$ 690,900}{\$ 600,900} \\
\end{array}$ & 2007 & $\begin{array}{l}5690,900 \\
5690,900\end{array}$ & $\begin{array}{l}0 \text { ostrm.PRD-700 } \\
\text { ostrm.PRD- } 600\end{array}$ & 23322.9925 & $\mathrm{~kg} / \mathrm{hr} / \mathrm{hr}$ & 0.65 & $\frac{1.1850}{1.850}$ & $\frac{10,798}{13,871}$ & 0.46 & $\$ 4492,819$ & 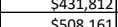 & $\begin{array}{r}\$ 798,852 \\
\$ 900 \\
\end{array}$ \\
\hline $\begin{array}{l}\text { Glucose Storage Tank } \\
\text { Glo }\end{array}$ & $70,000 \mathrm{gal}$ & & Glass lined & 1 & $\begin{array}{l}\$ 500,900 \\
570,000 \\
\end{array}$ & 2009 & $\begin{array}{ll}5600,900 \\
570,000\end{array}$ & of strm. 4400.401 & & $\mathrm{~kg} / \mathrm{hr}$ & 0.70 & $\frac{1.850}{2.6}$ & 1,557 & $\frac{0.59}{1.12}$ & $\frac{\$ 425,8106}{\$ 75,686}$ & $\begin{array}{ll}\$ 58,161 \\
\$ 78,557 \\
\end{array}$ & $\begin{aligned} \$ \$ 40,0097 \\
204,249 \\
\end{aligned}$ \\
\hline & & & & & & & & & & & & & & 900 Totals & $\$ 9,861,551$ & $\$ 9,894,800$ & $\$ 17,195,641$ \\
\hline
\end{tabular}


BDO Pathway

\begin{tabular}{|c|c|c|c|c|c|c|c|c|c|c|c|c|c|c|c|c|c|}
\hline \multirow[b]{2}{*}{$\begin{array}{c}\text { A200: Pretreatment } \\
\text { EQUIPMENT TITLE }\end{array}$} & \multicolumn{5}{|c|}{ Mechanical Equipment List } & \multicolumn{12}{|c|}{ Scaled Installed Costs } \\
\hline & DESCRIPTION & HP & MATERIAL & 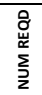 & $\$$ & 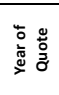 & $\begin{array}{c}\text { Purch Cost in } \\
\text { Base } \mathrm{Yr}\end{array}$ & Scaling Variable & 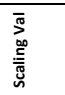 & $\frac{\underline{n}}{5}$ & 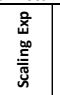 & 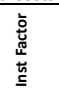 & 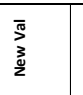 & 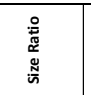 & $\begin{array}{c}\text { Scaled Purch } \\
\text { cost }\end{array}$ & $\begin{array}{l}\text { Purch Cost in } \\
\text { Proj year }\end{array}$ & $\begin{array}{l}\text { Inst Cost in } \\
\text { Proj year }\end{array}$ \\
\hline Flash Tank Agitator & Side-mounted, $3 \times 75$ hp. $(170 \mathrm{~kW})$ & $170 \mathrm{~kW}$ & $31615 S$ & 3 & $\$ 90,000$ & 2009 & $\$ 90,000$ & Strm.a200.254 & 252891 & $\mathrm{~kg} / \mathrm{hr}$ & 0.50 & 1.5 & 192,146 & 0.76 & $\$ 78,450$ & $\$ 81,426$ & $\$ 122,139$ \\
\hline Ammonia Addition Tank Agitator & & $10 \mathrm{hp}$ & ss & 1 & $\$ 21,900$ & 2009 & $\$ 21,900$ & Strm.a200.228 & 410369 & $\mathrm{~kg} / \mathrm{hr}$ & 0.50 & 1.5 & \begin{tabular}{|c|c|}
230,647 \\
2,60
\end{tabular} & 0.56 & $\$ 16,418$ & $\$ 17,041$ & $\begin{array}{ll}\$ 25,562 \\
, 566 \\
\end{array}$ \\
\hline Ammonia Static Mixer & & & ss & 1 & $\begin{array}{ll}\$ 5,000 \\
\$ \$ 2000\end{array}$ & 2009 & \begin{tabular}{|l|l|}
55,000 \\
59001
\end{tabular} & strm.a200.275 & 157478 & $\mathrm{~kg} / \mathrm{hr}$ & 0.50 & 1.0 & \begin{tabular}{|l|l|}
38,500 \\
829
\end{tabular} & 0.2445 & 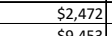 & \begin{tabular}{l|l}
$\$ 2,566$ & \\
50207 & \\
5
\end{tabular} & \begin{tabular}{|l}
$\$ 2,566$ \\
$\$ 2,53$
\end{tabular} \\
\hline $\begin{array}{l}\text { Pretreatment Water Heater } \\
\text { Pretreatment }\end{array}$ & 29.9 MMBtu & & 30455 & 1 & $\frac{\$ 92,000}{\$ 5,42,400}$ & $\frac{2010}{2010}$ & $\begin{aligned} 592,000 \\
552000\end{aligned}$ & $\begin{array}{l}\text { Heat.A200.QH201 } \\
\text { SCISO200 DAC.19 }\end{array}$ & $\begin{array}{r}-8 \\
3860 \\
\end{array}$ & Gcal/hr & 0.70 & 2.2 & \begin{tabular}{|c|c|}
$(0.29)$ \\
7083
\end{tabular} & 0.04 & $\begin{aligned} \$ 9,453 \\
\$ 904107 \\
\end{aligned}$ & $\begin{aligned} \$ 9,297 \\
\$ 97055 \\
\end{aligned}$ & $\begin{aligned} \$ 20,453 \\
5970555 \\
\end{aligned}$ \\
\hline $\begin{array}{l}\text { Pretreatment sugar Beet extruder } \\
\text { Milling Equipment }\end{array}$ & $200 \mathrm{kw} / \mathrm{dry}$ ton & & & $\frac{1}{8}$ & $\$ \$ \$ \$, 424,4000$ & 2010 & $\begin{array}{l}\$ 5,424,000 \\
\$ 2,466,700\end{array}$ & $\begin{array}{l}S \text { SII.Sa200.DACAC-IN } \\
\text { SIS.a200.211b }\end{array}$ & 38600 & $\mathrm{~kg} / \mathrm{kg} / \mathrm{hr}$ & $\frac{1.000}{0.60}$ & $\frac{1.0}{1.5}$ & $\begin{array}{l}0,0783 \\
57,306\end{array}$ & $\frac{1.83}{0.91}$ & $\begin{aligned} \$ 19,794,18,600 \\
\$ 19,733,60\end{aligned}$ & $\begin{array}{r}\$ 9,740,555 \\
\$ 18,843,101\end{array}$ & $\begin{aligned} \$ 28,400,535 \\
\$ 28,264,651\end{aligned}$ \\
\hline Milling Equipment-Szego Mill & & & & 11 & $\$ 578,000$ & 2013 & $\frac{\$ 2,400,100}{\$ 578,000}$ & ScIs.a200.211b & 62942 & $\mathrm{~kg} / \mathrm{hr}$ & 0.60 & 1.4 & 57,306 & 0.91 & \$1 & \begin{tabular}{|l|l|}
$56,041,089$ \\
5671
\end{tabular} & $\$ 8,499,524$ \\
\hline Blowdown Tank Discharge Pump & 1900 GPM, 150 FT TDH & 125 & 31655 & 1 & $\$ 25,635$ & 2010 & $\$ 25,635$ & strm.a200.222 & 292407 & $\mathrm{~kg} / \mathrm{hr}$ & 0.80 & 2.3 & 193,144 & 0.66 & $\$ 18,397$ & $\$ 18,093$ & $\frac{541,614}{5414}$ \\
\hline Flash Tank Discharge Pump & $900 \mathrm{GPM}, 150 \mathrm{FT} \mathrm{TDH}$ & 75 & $3165 s$ & 1 & $\$ 30,000$ & 2009 & $\$ 30,000$ & strm.a200.254 & 204390 & $\mathrm{~kg} / \mathrm{hr}$ & 0.80 & 2.3 & 192,146 & 0.94 & $\$ 28,554$ & $\$ 29,637$ & $\$ 68,165$ \\
\hline Deacetylation Tank Discharge Pump & 1771 GPM, 150 FT TDH & 100 & 31655 & 1 & $\$ 22,500$ & 2009 & $\$ 22,500$ & strm.a200.211a & 402194 & $\mathrm{~kg} / \mathrm{hr}$ & 0.80 & 2.3 & 283,665 & 0.71 & $\$ 17,017$ & $\$ 17,662$ & $\$ 40,624$ \\
\hline Hydrolyzate Pump & 1771 GPM, 150 FT TDH & 100 & 31655 & 1 & $\$ 22,500$ & 2009 & $\$ 22,500$ & strm.a200.228 & 402194 & $\mathrm{~kg} / \mathrm{hr}$ & 0.80 & 2.3 & 230,647 & 0.57 & $\$ 14,421$ & \$14,968 & $\$ 34,426$ \\
\hline Deacetylation reactor conveyors & Feed and discharge drag conveyors & $40 \mathrm{hp}$ & 55316 & 3 & $\$ 110,000$ & 2013 & $\$ 110,000$ & strm.a200.211a & 277167 & $\mathrm{~kg} / \mathrm{hr}$ & 0.80 & 1.7 & 283,665 & 1.02 & $\$ 336,175$ & $\$ 321,004$ & $\$ 545,707$ \\
\hline S/L Split Discharge Pump to WWT & 900 GPM, 150 FT TDH & 75 & 31655 & 1 & $\$ 30,000$ & 2009 & $\$ 30,000$ & strm.a200.4 & 204390 & $\mathrm{~kg} / \mathrm{hr}$ & 0.80 & 2.3 & 193,144 & 0.94 & $\$ 28,672$ & $\$ 29,760$ & $\begin{array}{l}\$ 68,447 \\
08,201\end{array}$ \\
\hline Flash Tank & 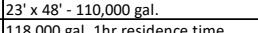 & & $\frac{5 S 316}{5 \$ 334}$ & 1 & $\frac{\$ 511,000}{\$ 236000}$ & $\frac{2009}{2009}$ & $\begin{array}{l}\$ 511,000 \\
\$ 230000\end{array}$ & strm.a200.2233 & 264116 & gkg/hr & 0.70 & 2.0 & $\mid 193,144$ & 0.73 & $\$ 410,470$ & $\frac{\$ 426,042}{516567}$ & $\begin{aligned} \$ 852,084 \\
527706\end{aligned}$ \\
\hline Ammonia Addition Tank & $118,000 \mathrm{gal}, 1 \mathrm{hr}$ residence time & & 55304 & 1 & $\$ 236,000$ & 2009 & $\$ 236,000$ & strm.a200.228 & 410369 & $\mathrm{~kg} / \mathrm{hr}$ & 0.70 & 2.0 & $230,647]$ & 0.56 & $\$ 157,671$ & $\$ 163,653$ & $\$ 327,306$ \\
\hline & & & & & & & & & & & & & & 200 Totals & $\$ 37,113,956$ & $\$ 35,785,895$ & $\$ 48,653,825$ \\
\hline
\end{tabular}

\begin{tabular}{|c|c|c|c|c|c|c|c|c|c|c|c|c|c|c|c|c|c|}
\hline \multirow{2}{*}{$\begin{array}{c}\text { A300: Hydrolysis and Fermentation } \\
\text { EQUIPMENT TIILE }\end{array}$} & \multicolumn{5}{|c|}{ Mechanical Equipment List } & \multicolumn{12}{|c|}{ Scaled Installed Costs } \\
\hline & DESCRIPTION & HP & MATERIAL & 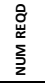 & $\$$ & 要 & 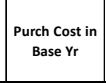 & Scaling Variable & 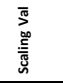 & $\frac{u^{2}}{5}$ & 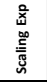 & 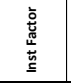 & 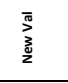 & 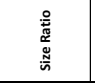 & Scaled Purch cost & $\begin{array}{c}\text { Purch Cost in Proj } \\
\text { year }\end{array}$ & Inst cost in Proj year \\
\hline $\begin{array}{l}\text { Batch Ennymatic Hodrolysis } \\
\text { Hydrolyzate cooler }\end{array}$ & Plate \& Frame $32.5 \mathrm{MMBt} / \mathrm{hr}$ & & SS 304 & 1 & 585,000 & & $\$ 85,000$ & heat.A300.E. F.CC301 & & Gcal/hr & 0.70 & & & & & $\begin{array}{l}569,941 \\
\end{array}$ & $\$ 153,871$ \\
\hline $\begin{array}{l}\text { Enzyme-Hydrolysate Mixer } \\
\text { Sacharififation Tank }\end{array}$ & 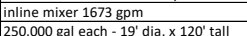 & $100 \mathrm{hp}$ & 55316 & $\frac{1}{8}$ & $\begin{array}{l}\$ 109,000 \\
\$ 8,000\end{array}$ & 2009 & & 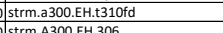 & 37993 & kg/hr & 0.50 & 1.7 & & 0.00 & & & 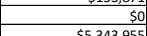 \\
\hline $\begin{array}{l}\text { SScchar rification Tank } \\
\text { Saccharification Transfer Pump }\end{array}$ & 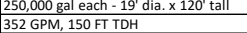 & 20 & $\begin{array}{l}30455 \\
31655 \\
\end{array}$ & $\frac{8}{5}$ & & $\frac{2009}{2009}$ & $\begin{array}{r}\$ 3,840,000 \mid \\
\$ \$ 7,200\end{array}$ & stsm.A300.EH.306 & $\begin{array}{l}421776 \\
422776\end{array}$ & $\mathrm{~kg} / \mathrm{hr} / \mathrm{hr}$ & 0.70 & $\begin{array}{r}2.0 \\
2.3 \\
\end{array}$ & $\frac{238,222}{238,22}$ & 0.56 & 2574312.5936 & $\frac{\$ 2,671,978}{\$ 31019}$ & $\begin{array}{l}\$ 5,343,955 \\
\$ 711345\end{array}$ \\
\hline 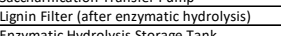 & 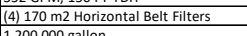 & $660 \mathrm{hp} \mathrm{ea}$ & 30455 & 0 & $\frac{\$ 2,152,500}{22,506}$ & 2013 & & 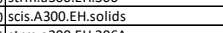 & 328984 & $\frac{d g h t h r}{k g / h r}$ & 1.00 & 1.7 & & $\frac{1.00}{1.00}$ & $\begin{aligned} 50 \\
50\end{aligned}$ & 50 & 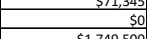 \\
\hline $\begin{array}{l}\text { Enzymatic Hydrolysis Storage Tank } \\
\text { Hydrolyzate Storage Transffer Pump }\end{array}$ & 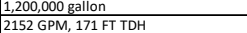 & 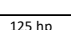 & $\frac{31655}{31555}$ & $\frac{1}{1}$ & $\frac{\$ 1,317,325}{\$ 26800}$ & $\frac{2011}{2009}$ & $\frac{\$ 1,317,325}{\$ \$ 6,800}$ & $\begin{array}{l}\text { Ststr.a300.EH.306A } \\
\text { strmm.A300.PROD-EH }\end{array}$ & $\frac{328984}{488710}$ & kg/hr $\mathrm{kg} / \mathrm{hr}$ & 0.70 & $\frac{1.8}{2.3}$ & $238,22$. & 0.72 & $\$ 1,050,891 \mid$ & $\$ 971,944$ & $\begin{aligned} 51,79,500 \\
50\end{aligned}$ \\
\hline Fittered Hydrolysate Storage Tank & 30,000 gallons -20 min residence time & $\frac{120 \mathrm{hp}}{20 \mathrm{hp}}$ & $\frac{31053}{55}$ & $\frac{1}{1}$ & $\frac{32,0,000}{551,600}$ & 2011 & $\begin{array}{l}\$ 20,800 \\
\$ 51,600 \\
\end{array}$ & strm.A300.PROD-EH & $3 \frac{487890}{34860}$ & $\mathrm{~kg} / \mathrm{hr}$ & $\frac{0.00}{0.70}$ & 2.3 & & 0.00 & & & $\begin{aligned} 50 \\
50 \\
\end{aligned}$ \\
\hline ermentor Tank (saccharification contributio & & & 30455 & 12 & $\$ 10,128,000$ & 2009 & $\$ 10,128,000$ & NVESBDO & & ea & 1 & 1.50 & & 0.476 & $\$ 4,817,202$ & $\$ 4,999,959$ & $\$ 7,499,938$ \\
\hline Fermentor Aigitator (saccharifification contribit & ion) & $30 \mathrm{hp}$ & 55304 & 1 & $\frac{\$ 52,500}{\$ 856928}$ & 2009 & $\begin{array}{l}\$ 52,500 \\
\$ 89028 \\
\end{array}$ & NNESBDO & & ea & 1 & 1.50 & & 5.708 & 5299,648 & $\$ 311,016$ & 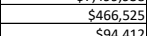 \\
\hline 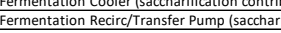 & 340 GPM, 150 FT & 20 & $\begin{array}{l}30455 \\
31655 \\
\end{array}$ & $\frac{12}{5}$ & $\frac{\frac{856,928}{547,200}}{5}-19$ & 20099 & $\begin{array}{l}58,69280 \\
\$ 47,200\end{array}$ & $\begin{array}{l}\text { NVESBDD } \\
\text { NVESBDO }\end{array}$ & & lea & $\frac{1}{0.8}$ & 2.200 & & $\begin{array}{l}0.476 \\
0.476\end{array}$ & $\begin{array}{l}\$ 4,13466 \\
\$ 22,047\end{array}$ & $\begin{array}{l}\$ 42,1944 \\
\$ 227,035\end{array}$ & $\begin{array}{l}\$ 9,412 \\
\$ 62,181\end{array}$ \\
\hline & & & & & & & & & & & & & & 300 Totals & $\$ 8,910,449$ & $\begin{array}{l}9,125,808 \\
\end{array}$ & $\$ 15,441,726$ \\
\hline
\end{tabular}

\begin{tabular}{|c|c|c|c|c|c|c|c|c|c|c|c|c|c|c|c|c|c|}
\hline \multirow{2}{*}{$\begin{array}{c}\text { A400:Enzyme Production } \\
\text { EquPMENT TITLE }\end{array}$} & \multicolumn{5}{|c|}{ Mechanical Equipment List } & \multicolumn{12}{|c|}{ Scaled Installed Costs } \\
\hline & DESCRIPTION & HP & MATERIAL & 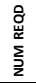 & $\$$ & 吾 & 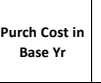 & Scaling Variable & 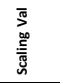 & $\frac{2}{5}$ & 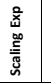 & 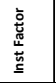 & $\begin{array}{l}\frac{\bar{g}}{3} \\
\frac{3}{2} \\
\frac{3}{2}\end{array}$ & 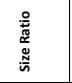 & Scaled Purch Cost & $\begin{array}{c}\text { Purch Cost in Proj } \\
\text { year }\end{array}$ & Inst cost in Proj year \\
\hline Cellulase Fermentor Agitators & & 800.0 & 55316 & & $\$ 580,000$ & 2009 & $\$ 580,0000$ C & CLVESSEL & & ea & 1.00 & & & 5.00 & & $\$ 3,010,0211$ & \\
\hline 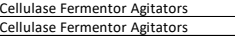 & & $0.7 \mathrm{hp}$ & 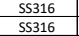 & & $\frac{\$ 3,420}{\$ 1,1,000}$ & $\frac{2009}{2009}$ & 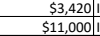 & $\begin{array}{l}\text { IILLEED } \\
\text { ICLEEDD }\end{array}$ & & ea & $\frac{1.00}{1.00}$ & $\frac{1.5}{1.5}$ & & $\begin{array}{l}4.00 \\
4.00\end{array}$ & $\begin{array}{l}\frac{513,680}{544,000} \\
\end{array}$ & $\begin{array}{ll}\$ 14,499 \\
\$ 45,6999 \\
\end{array}$ & $\begin{array}{ll}5212,298 \\
568,504 \\
\end{array}$ \\
\hline Cellulase Fermentor Agitators & & $80 \mathrm{hp}$ & 553316 & & 563,000 & 2009 & $\frac{563,000}{5600}$ & ICLSEED & & ea & 1.00 & 1.5 & & 4.00 & $\$ 252,000$ & $\$ 261,560$ & $\frac{5392,341}{\$ 3}$ \\
\hline $\begin{array}{l}\text { Medial-Prep Tank Agititotor } \\
\text { Cellulase Nutrient }\end{array}$ & & $7.5 \mathrm{hp}$ & $\frac{A 285 \mathrm{C}}{\mathrm{cs}}$ & 1 & $\frac{58,500}{5400}$ & $\frac{2009}{2009}$ & $\frac{58,500 \mid}{54800 \mid} \mid$ & 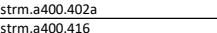 & $\frac{12255}{174}$ & kg/hr & 0.50 & $\frac{1.5}{1.6}$ & & 0.00 & $\begin{aligned} 50 \\
54030\end{aligned}$ & $\begin{aligned} 50 \\
55100 \\
\end{aligned}$ & $\begin{aligned} 50 \\
5697 \\
5697\end{aligned}$ \\
\hline 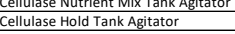 & & $\frac{3 \mathrm{hp}}{10 \mathrm{hp}}$ & $\frac{\mathrm{cs}}{5 S 316}$ & $\frac{1}{1}$ & $\begin{aligned} \$ \$, 400 \\
\$ 26,900\end{aligned}$ & $\frac{2009}{2009}$ & 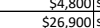 & strm.4400.4216 & 10930 & kg/hr & 0.50 & $\frac{1.6}{1.5}$ & $\frac{123}{7,575}$ & $\frac{0.70}{0.69}$ & $\left.\frac{54,030}{522395}\right]$ & $\frac{54,182}{523,244}$ & $\begin{array}{c}\$ 6,692 \\
534866\end{array}$ \\
\hline & $80,000 \mathrm{gal}, 1 \mathrm{~atm}, 28^{\circ} \mathrm{C}$, Internal coil & & S5s316 & & $\begin{array}{lll}\$ 20,00,500 \\
\$ 400,500\end{array}$ & 2009 & $\frac{S 2,90,000}{5400,500}$ & CLIVESSEL & 1 & ea & 1.00 & 2.0 & & 5.00 & $\frac{52,395}{\$ 2,02,500}$ & $\frac{523,244}{\$ 2,078,471}$ & $\frac{544,860}{45,156,943}$ \\
\hline st Cellulase Seed Fermentor & 80 gallon skid complete - $\$ 46,000$ ea & & 3045 & & 546,000 & 2009 & 546,000 年 & ICLSEED & & ea & 1.00 & 1.8 & & 4.00 & 5184,000 & $\$ 190,9811$ & 5343,765 \\
\hline $\begin{array}{l}\text { 2nd Cellulase esed Fermentor } \\
\text { Brdd Cellulase Seed Fermentor }\end{array}$ & 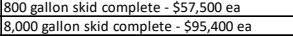 & & $\begin{array}{l}\frac{3045 s}{30455} \\
\end{array}$ & & $\frac{557,500}{595,400}$ & $\frac{2009}{2009}$ & $\frac{\$ 57,500 \mid}{\$ 95,400 \mid}$ & $\begin{array}{l}\text { IISLEDD } \\
\text { ICLEED }\end{array}$ & & ea & $\frac{1.000}{1.00}$ & $\frac{1.8}{1.8}$ & & $\frac{4.00}{4.00}$ & \$ $\$ 230,000$ & 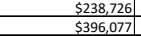 & 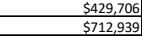 \\
\hline 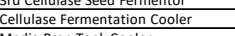 & 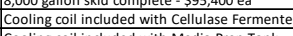 & & 30455 & & 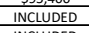 & & & & & & & & & & & & \\
\hline Media Prep Tank Cooler & 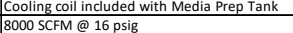 & & $\frac{3045}{c 5}$ & $\frac{1}{2}$ & $\frac{\text { INCLLDED }}{\$ 350,000}$ & & & Strm. .4000 .450 & & & 0.001 & 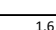 & & & & 5250,395 & 5400,632 \\
\hline 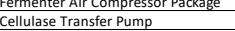 & 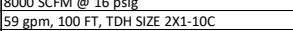 & 3 & $\frac{\mathrm{cs}}{3165}$ & $\frac{2}{1}$ & $\frac{5300,00}{57,357}$ & $\frac{2009}{2010}$ & $\frac{530,000 \mid}{57,357 !}$ & 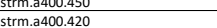 & $\frac{33168}{13399} \mathrm{rall}$ & $\frac{k g / h / h}{\mathrm{~kg} / \mathrm{hr}}$ & $\frac{0.60}{0.80}$ & $\frac{1.6}{2.3}$ & $\frac{1,7899}{7,575}$ & $\frac{0.54}{0.57}$ & 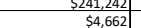 & S250,0955 & $\begin{array}{l}\$ 40,0,622 \\
\$ 10,545\end{array}$ \\
\hline ellulase Seed Pump & 3 GPM, 100 FT TDH SIZE $2 \times 1-10$ & 2 & 31655 & 4 & 529,972 & 2010 & $\$ 29,972$ & Strm.a400.409 & $\frac{151}{681}$ & $\mathrm{~kg} / \mathrm{hr}$ & 0.80 & $\frac{2.3}{2.3}$ & 421 & 0.62 & 520,408 & $\$ 20,071$ & $\begin{array}{ll}\$ 1,0453 \\
\$ 46,163\end{array}$ \\
\hline ellulase Nutrient Transfer Pump & Gear Pump 2 GPM, 100 FT & 1 & 31655 & 1 & $\$ 1,500$ & 2009 & $\$ 1,500$ & Strm. .4000 .416 & 454 & $\mathrm{~kg} / \mathrm{hr}$ & 0.80 & & 123 & 0.27 & $\$ 526$ & 5546 & \\
\hline $\begin{array}{l}\text { Eellulase Feed Pump } \\
\text { Entitiom Pump }\end{array}$ & $\begin{array}{l}\text { Gear Pump } \\
\text { Gear Pump } 2 \text { GPM, } 100 \text { FT }\end{array}$ & 1 & $\frac{31655}{31955}$ & 1 & $\$ 5,700$ & 2009 & $\begin{array}{c}\$ 5,700 \\
\$ \$ 1500\end{array}$ & Strm.a400.422 & 18168 & klggh & 0.80 0 & 2.3.3 203 & 7,575 & 0.42 & $\$ 2,831$ & $\$ 2,938 \mid$ & $\frac{\$ 6,759}{5261}$ \\
\hline 'elllulase Numprient Mix Tank & HDFE 8.000 gal & 1 & $\frac{31655}{\text { HOPE }}$ & 1 & $\begin{array}{l}\frac{51,500}{59,000} \\
-15\end{array}$ & $\frac{2009}{2010}$ & & $\begin{array}{ll}\text { Stsm.m.a4000.444 } \\
\text { Strm. } .400 .416\end{array}$ & & 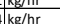 & 0.00 & & & .0.55 0.55 & 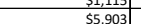 & 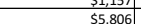 & 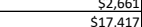 \\
\hline Eellulase Hold Tank & $80,000 \mathrm{gal}$ & & 30455 & 1 & $\$ 248,070$ & 2009 & & Strm.a400.422 & & $\mathrm{kg} / \mathrm{hr}$ & 0.70 & & & 0.69 & 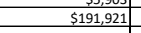 & $\frac{53,300}{5199,202}$ & 3018,564 \\
\hline & & & & & & & & & & & & & & 400 Totals & $\$ 6,502,813$ & $\$ 6,747,832$ & $\$ 11,526,084$ \\
\hline
\end{tabular}




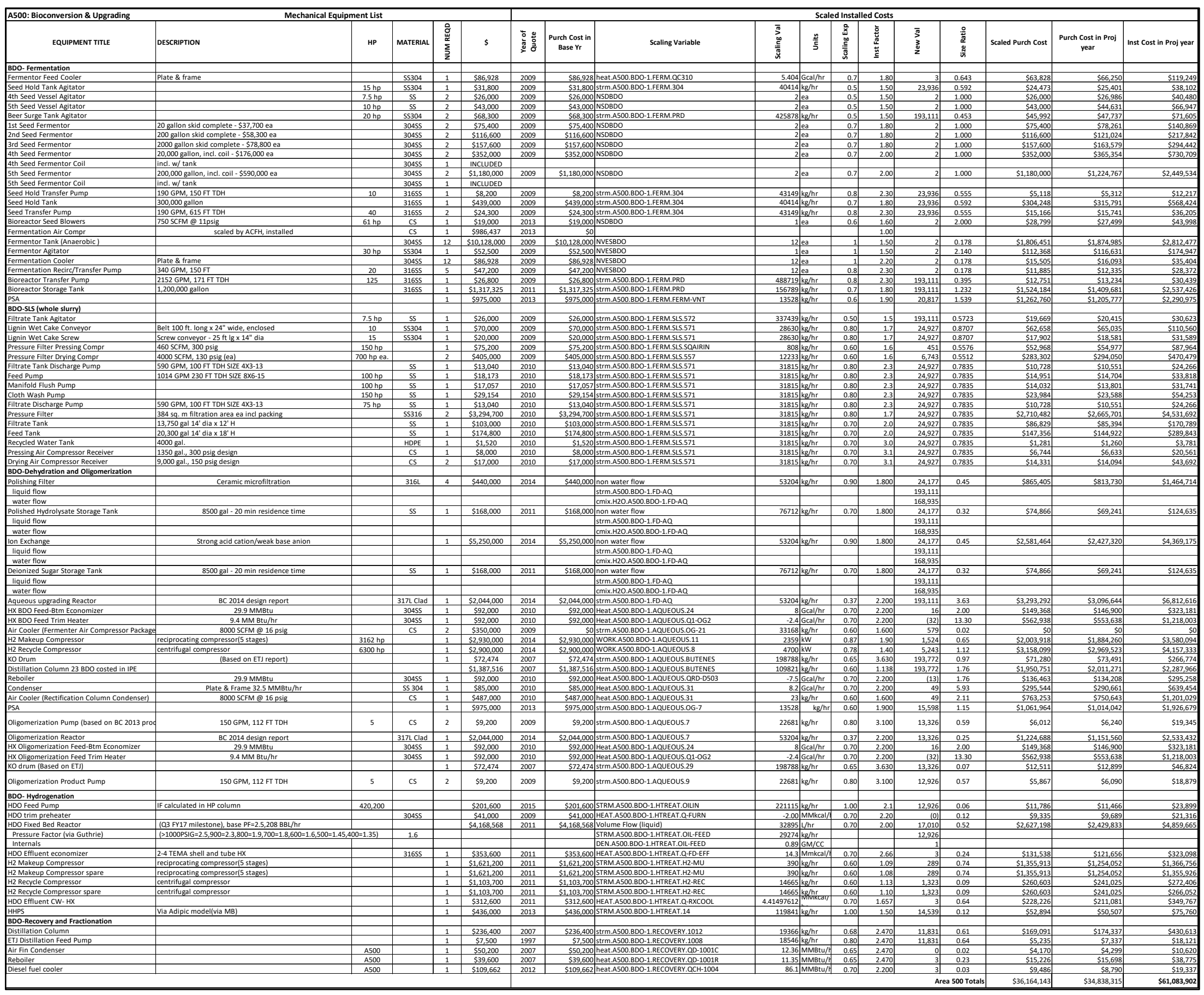




\begin{tabular}{|c|c|c|c|c|c|c|c|c|c|c|c|c|c|c|c|c|c|}
\hline \multirow[b]{2}{*}{\begin{tabular}{|c|} 
A600: WWT \\
EQUPMENT TITLE \\
\end{tabular}} & \multicolumn{5}{|l|}{ Mechanical Equipment List } & \multicolumn{12}{|c|}{ Scaled Installed Costs } \\
\hline & DESCRIPTION & HP & MATERIAL & 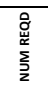 & $\$$ & 蒿 & $\begin{array}{c}\text { Purch Cost in } \\
\text { Base } \mathrm{Yr}\end{array}$ & Scaling Variable & 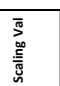 & $\frac{2}{5}$ & 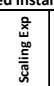 & 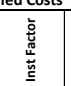 & $\begin{array}{l}\frac{\bar{m}}{3} \\
\frac{3}{2} \\
\frac{3}{2}\end{array}$ & 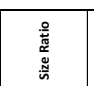 & Scaled Purch cost & $\begin{array}{l}\text { Purch Cost ti proj } \\
\text { year }\end{array}$ & Inst Cost in Proj year \\
\hline Aeration Basin & Concrete and steel, not installed cost & & Concrete & 3 & $\$ 4,804,854$ & 2012 & $54,804,854$ & Aydraulic flow & 2. & MGD & 0.60 & 2.1 & & 0.81 & $\$ 4,236,252$ & $\$ 3,925,381$ & $\$ 8,125,539$ \\
\hline 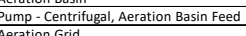 & $\begin{array}{l}852 \mathrm{gpm} \text { ea } \\
\text { Futf floor aration prid }\end{array}$ & $45 \mathrm{hp}$ & $\begin{array}{cl}\mathrm{cs} \\
\mathrm{cs}\end{array}$ & $\frac{4}{1}$ & $\frac{\$ 64,800}{52500000}$ & $\frac{2012}{2012}$ & & & & & & & & & & 33,9203,384] & \\
\hline Caustic Feed System & & $1.5 \mathrm{hp}$ & $\frac{\mathrm{cs}}{\mathrm{cs}} \mathrm{l}(\mathrm{l})$ & $\frac{1}{4}$ & $\frac{35,50,000}{520,000}$ & $\frac{2012}{2012}$ & 520,000 & COD & 5600 & $\mathrm{~kg} / \mathrm{hr}$ & 0.60 & 3.0 & $7,128$. & 1.27 & $\$ 23,115$ & $\$ 21,419$ & 564,257 \\
\hline $\begin{array}{l}\text { Blowers } \\
\text { Mimbrane Bioreactor }\end{array}$ & 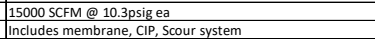 & 1000 hpea & $\frac{c s}{c s}$ & 9 & $\begin{array}{l}\$ 2,270,000 \\
\$, 489,500\end{array}$ & $\frac{2012}{2012}$ & $\begin{array}{ll}52,070,0000 \\
4,898,500\end{array}$ & $\begin{array}{c}\text { COD } \\
\text { Hydraulic flow }\end{array}$ & $\begin{aligned} 5600 \\
2.7\end{aligned}$ & 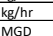 & $\begin{array}{l}0.60 \\
1.00 \\
\end{array}$ & 2.0 & 7,128 & $\frac{1.27}{0.81}$ & $\begin{array}{l}52,392,448 \\
53990.993\end{array}$ & \begin{tabular}{|l|l|}
$\$ 2,216,881$ \\
33,699588 \\
\end{tabular} & 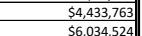 \\
\hline 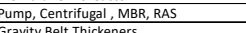 & & $160 \mathrm{hp}$ & & $\frac{1}{6}$ & & & & & & & & & & & & & \\
\hline $\begin{array}{l}\text { Gevatitr Belt Thickeners } \\
\text { Centrifuge }\end{array}$ & 2 presses & $\frac{48 \mathrm{hp}}{105 \mathrm{hpea}}$ & $\frac{c s}{c s}$ & $\frac{3}{1}$ & $\begin{array}{l}5750,000 \\
5656,800\end{array}$ & $\frac{2012}{2012}$ & $\begin{array}{ll}5750,000 \\
5680.800\end{array}$ & COD & $\frac{5600}{5600}$ & $\mathrm{~kg} / \mathrm{hr}$ & $\frac{0.60}{0.60}$ & $\frac{1.6}{2.7}$ & $\begin{array}{cc}7,128 \\
77128\end{array}$ & $\frac{1.27}{1.27}$ & $\begin{array}{ll}586,829 \\
597,784\end{array}$ & $\begin{array}{ll}5803,218 \\
575533 \\
\end{array}$ & 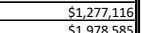 \\
\hline Pump, centrifitugal, Centrifiuge Feed & $105 \mathrm{gpm}$ & $\frac{1565 \mathrm{~h} \mathrm{ea}}{15 \mathrm{hp}}$ & cs & $\frac{1}{2}$ & $\begin{array}{l}\frac{3668,800}{\text { INCLOUED }} \\
\end{array}$ & 2012 & & & & & & & & & & & \\
\hline 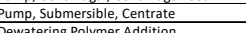 & $100 \mathrm{gpm}$ & $10 \mathrm{hpea}$ & cs & 2 & $\begin{array}{ll}\text { INCLLDEE } \\
\text { ICUES }\end{array}$ & 2012 & & & & & & & & & & & \\
\hline $\begin{array}{l}\text { Dewatering Polymer Addition } \\
\text { Conveyor }\end{array}$ & $9.8 \mathrm{ghh} \mathrm{neat} \mathrm{polymer}$ & $\frac{1 \text { hpea }}{1 \text { hopea }}$ & $\frac{c s}{c s}$ & $\frac{2}{1}$ & $\frac{\text { INCLUDED }}{\text { STO000 }}$ & $\frac{2012}{2012}$ & 57000 & & 5600 & $\mathrm{k} / \mathrm{hr}$ & $0.60 \mathrm{~d}-\mathrm{C}>\mathrm{C}$ & $2.97>>20$ & 7,128 & 1.27 & 58.090 & & 521.441 \\
\hline $\begin{array}{l}\text { Reverse Osmosis } \\
\text { Rearotar }\end{array}$ & & & & 7 & 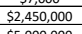 & 2012 & $\begin{array}{l}52,450,000 \\
5<5000\end{array}$ & tydraulic flow & & $M G D$ & 1.00 & $\frac{2.9}{1.8}$ & & 0.81 & $\begin{array}{ll}51,986,105 \\
\end{array}$ & $51,840,357$ & 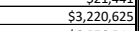 \\
\hline $\begin{array}{l}\text { Evaporatar } \\
\text { Ammonia Addition System }\end{array}$ & $\begin{array}{ll}\frac{366 \mathrm{gpm}}{0.63 \mathrm{gpm}} \\
\end{array}$ & $\begin{array}{ll}1480 \mathrm{hppa} \\
4.5 \mathrm{hp}\end{array}$ & Titanium & $\frac{1}{4}$ & $\frac{\$ 5,00,0,000}{5195,200}$ & $\frac{2012}{2012}$ & $\begin{array}{c}\frac{55,000,000}{5195,200} \\
5\end{array}$ & & $\frac{200}{5600}$ & $\begin{array}{lll}\mathrm{MGD} \\
\mathrm{kg} / \mathrm{hr}\end{array}$ & $\begin{array}{l}0.60 \\
0.60 \\
\end{array}$ & $\frac{1.6}{1.5}$ & $\frac{2}{7,128}$ & $\frac{0.81}{1.27}$ & $\frac{54,408,305}{5225,607}$ & 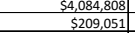 & $\frac{\$ 6,576,5411}{5321,938}$ \\
\hline $\begin{array}{l}\text { Sodium Sulfate Purfification } \\
\text { Svaporatoto feed dank }\end{array}$ & [insulated, 6460 gal & & & & 545.966 & 2011 & & titm.A600.23 & & & 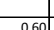 & & & 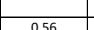 & & & \\
\hline 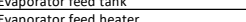 & & & & & & & 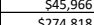 & & & 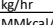 & 0.60 & 年, 300 & $161,490 \mid$ & $\begin{array}{l}0.56 \\
0.35\end{array}$ & & 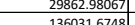 & $\frac{\$ 74,657}{5508095}$ \\
\hline Evaporator flash drum & 13. $13^{\prime} \times 48^{\prime}-110,000$ gal. & & 55316 & 1 & 5511,000 & 2009 & 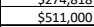 & titm.4600.23 & $\frac{-13}{264116}$ & $\mathrm{~kg} / \mathrm{hr}$ & 0.70 & 2.00 & 161,490 & 0.01 & $\begin{array}{cccc}514,701 \\
\$ 362,132\end{array}$ & 375870.7115 & 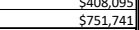 \\
\hline & 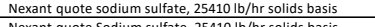 & & & 1 & $\begin{array}{l}5327,680 \\
\$ 555000\end{array}$ & 2011 & $\frac{5327,680}{655600}$ & Strm.A600.NA2504 & & $\mathrm{kg} / \mathrm{hr}$ & 0.60 & 2.3 & 14,871 & & 5381,855 & $\$ 353,169$ & 5812,288 \\
\hline & 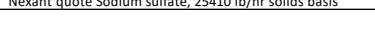 & & & 1 & & \multicolumn{9}{|c|}{$\begin{array}{ll}14,1031 & 1.23 \\
\text { Area } 600 \text { Totals }\end{array}$} & $\begin{aligned} \$ 520,48,1089 \\
20,92\end{aligned}$ & $\begin{aligned} 5850,924 \\
\$ 18,999,589\end{aligned}$ & 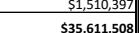 \\
\hline A700: Lignin Utilization & \multicolumn{5}{|c|}{ Mechanical Equipment List } & \multicolumn{12}{|c|}{ Scaled Installed Costs } \\
\hline EQUIPMENT TITLE & DESCRIPTION & HP & MATERIAL & 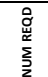 & $\$$ & 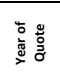 & $\begin{array}{c}\text { Purch Cost in } \\
\text { Base Yr }\end{array}$ & Scaling Variable & 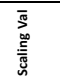 & $\frac{2}{5}$ & 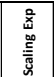 & 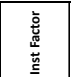 & $\begin{array}{l}\text { 焉 } \\
\frac{3}{2}\end{array}$ & 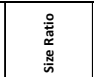 & Scaled Purch cost & $\begin{array}{l}\text { Purch Cost in Proj } \\
\text { year }\end{array}$ & Inst cost in Proj year \\
\hline 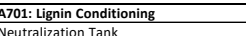 & 26 atm.130C operating $30 \mathrm{~min}$ hold $=30.000 \mathrm{pal}$ & & & & & & & Strm A700.A701 UOUD1 & & & & & & & & & \\
\hline 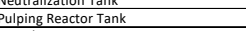 & 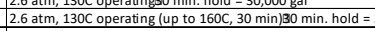 & ,000 gal & $\begin{array}{llll}55317 \\
55316\end{array}$ & $\frac{1}{1}$ & $\frac{2365,000}{516,300,000}$ & 2013 & 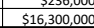 & 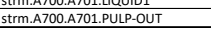 & $\frac{34036}{32329}$ & $\mathrm{~kg} / \mathrm{hr}$ & 0.70 & & $\frac{83,860}{117,840}$ & $\frac{0.20}{0.36}$ & $\begin{aligned} 57,76,187 \\
58,189\end{aligned}$ & $\begin{array}{r}\$ 8,404,739 \\
\$ 8,739\end{array}$ & $\frac{\$ 1611206}{\$ 14,016,319}$ \\
\hline Flash/drain tank & (1) & & $\frac{55317}{3.165}$ & $\frac{1}{1}$ & $\begin{array}{ll}5262,000 \\
51317325\end{array}$ & $\frac{2013}{2011}$ & $\begin{array}{rll}5262,000 \\
51317325\end{array}$ & 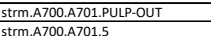 & $\frac{323296}{32989}$ & $\frac{1 \mathrm{~kg} / \mathrm{hr}}{\mathrm{kg} / \mathrm{hr}}$ & $\frac{0.70}{0.70}$ & 2.09 & $\frac{117,840}{92912}$ & 0.36 & $\begin{array}{lll}5129,267 \\
543652\end{array}$ & $\begin{array}{ccc}5123,433 \\
5502811\end{array}$ & $\begin{array}{c}5246,867 \\
505059 \\
\end{array}$ \\
\hline 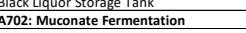 & & & & & & & & & & & & & & & & & $\$ 905,059$ \\
\hline st A Aerobic Seed Seed & 80 gallon skid complete $-546,000$ ea & $0.75 \mathrm{hp}$ & $\frac{30455}{55316}$ & - & $\frac{54,000}{5,320}$ & $\frac{2009}{2009}$ & $\begin{array}{cccc}545,000 \\
53,420\end{array}$ & $\frac{\text { NSS10000 }}{\text { NSDO1000 }}$ & & $\frac{\text { ea }}{\text { ea }}$ & $\begin{array}{l}1.00 \\
1.00 \\
-10\end{array}$ & $\frac{1.80}{1.50}$ & & $\frac{3.00}{3.00}$ & $\frac{5138,000}{51,260}$ & $\begin{aligned} 5143,2,35 \\
150,699 \\
\end{aligned}$ & $\begin{array}{l}\$ 257,824 \\
\$ 5,744\end{array}$ \\
\hline 2nd A Arobic Seed & 800 gallon skid complete $-\$ 57,500$ ea & $8 \mathrm{hp}$ & $\frac{3045}{55316}$ & & $\frac{\$ 57,500}{\$ \$ 11000}$ & 2009 & $\frac{\$ 57,500}{\$ 1.000}$ & NSS10000 & & $\frac{\mid e a}{\text { eaa }}$ & $\frac{1.00}{1.00}$ & $\begin{array}{l}1.80 \\
1.50 \\
-150\end{array}$ & & $\begin{array}{l}3.00 \\
3.00 \\
\end{array}$ & $\begin{array}{l}5172,500 \\
533,000 \\
\end{array}$ & $\begin{array}{ll}1179,044 \\
\$ \$ 34,2525 \\
\end{array}$ & $\frac{\$ 322,280}{\$ 551,178}$ \\
\hline Bubble column seed fermenter & $100 \mathrm{~m} 3$ & & & & 5274,100 & 2014 & 5274,100 & NSSD1000 & & ea & 1.00 & 2.30 & & 3.00 & $\$ 822,300$ & $\$ 773,199$ & $\$ 1,778,358$ \\
\hline $\begin{array}{l}\text { Seed circulation cooler } \\
\text { Bubble column production fermenter }\end{array}$ & $\begin{array}{lll}655 \text { soft } \\
1000 \mathrm{~m}^{3}\end{array}$ & & 31655 & & $\begin{array}{cl}58,400 \\
19691400\end{array}$ & 2014 & $\begin{aligned} 58,400 \\
519900\end{aligned}$ & 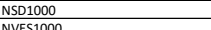 & & ea & 1.00 & 2.20 & & 3.00 & 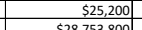 & $\begin{aligned} 523,695 \\
570,5957\end{aligned}$ & $\begin{aligned} 552,130 \\
52184772 \\
\end{aligned}$ \\
\hline Buble column production fermenter & 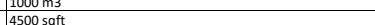 & & $\frac{365 s}{31655}$ & & $\frac{51,69,400}{58,100}$ & 年1044 & 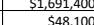 & 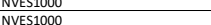 & & & $\frac{1.00}{100}$ & $\begin{array}{l}2.30 \\
220\end{array}$ & & $\begin{array}{l}17.00 \\
1700\end{array}$ & S28, $58,3,30000$ & 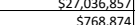 & 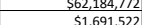 \\
\hline Production circulation pump & $400 \mathrm{gpm}$ & & 31655 & & 511,500 & 2014 & $\$ 11,500$ & NVES1000 & & ea & 1.00 & 2.30 & & 17.00 & 5195,500 & $\$ 183,826$ & 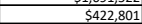 \\
\hline $\begin{array}{l}\text { Fermentation air compressor } \\
\text { Fermentation air receiver }\end{array}$ & $\begin{array}{l}25,0000 \text { A GFM @ 45psig; max size in ACCE } \\
225000 \text { al }\end{array}$ & & $\begin{array}{cc}c s \\
c s \\
c s\end{array}$ & & $\begin{array}{ll}51,318,600 \\
51006,60\end{array}$ & 2014 & $\frac{\$ 1,318,600}{\$ 1500600}$ & $\begin{array}{l}\text { ARR } 1000 \\
\text { ARV } 1000\end{array}$ & $\frac{13}{13}$ & $\frac{m{ }^{\wedge} 3 / 5}{m^{3} / 5}$ & $\frac{1.00}{1.00}$ & $\frac{1.60}{2.00}$ & $\frac{15}{15}$ & $\frac{1.14}{1.14}$ & $\frac{51,503,505}{5(112955}$ & $\begin{array}{ll}\$ \$ 1,414,052 \\
\$ 12,172 \\
\end{array}$ & $\frac{\$ 2,262,484}{\$ 224344}$ \\
\hline 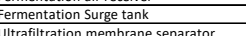 & insulated cone bottom, $6460 \mathrm{gal}$ & & - & & 5459,966 & 2011 & 545,966 & 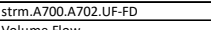 & 290932 & $\mathrm{~kg} / \mathrm{hr}$ & 0.60 & 2.50 & $162,59:$ & 0.56 & $\$ 32,421$ & $\$ 29,985$ & 574,963 \\
\hline $\begin{array}{l}\text { Ultrafiltration membrane separator } \\
\text { membrane broth feed uump }\end{array}$ & & & & & 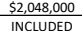 & 2011 & $52,048,000$ & $\begin{array}{l}\text { Volume Flow } \\
\text { Strm.A700.A702.UF-FD }\end{array}$ & & $\frac{\mathrm{GPM}}{\mathrm{k} / \mathrm{hr}}$ & 0.60 & 2.50 & $\frac{70}{162.59}$ & 1.00 & $\$ 2,048,000$ & $\$ 1,894,146$ & 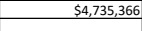 \\
\hline $\begin{array}{l}\text { membrane birth reea pump } \\
\text { membrane solvent feed pump }\end{array}$ & & & & & $\begin{array}{ll}\text { INACCUED } \\
\text { INCLOUDED }\end{array}$ & & & 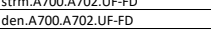 & & 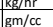 & & & & & & & \\
\hline A703: Recovery and Upgrading & & & & & & & & & & & & & & & & & \\
\hline $\begin{array}{l}\text { Carbon Filter } \\
\text { CMCM Crstallizer }\end{array}$ & \begin{tabular}{|l} 
2Vessels, for color removal \\
OSlso Type. 2 In series
\end{tabular} & & 31655 & 2 series & $\frac{5345,234}{57104,492}$ & $\frac{2011}{2011}$ & 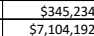 & $\begin{array}{l}\frac{l}{\text { Volume Flow }} \\
\text { Volume Flow }\end{array}$ & & $\frac{69 M}{G P M}$ & 0.600 & 2.50 & $\frac{64}{44}$ & $\frac{1.00}{0.21}$ & $\frac{5345,234}{\$ 5206,358}$ & $\begin{array}{l}319298.71757 \\
2595533.628\end{array}$ & $\frac{5798,247}{56488,834}$ \\
\hline 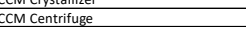 & $\begin{array}{l}\text { Centrifuege Separator } \\
\end{array}$ & & & & & $\frac{2011}{2011}$ & $\begin{aligned} 57,104,192 \\
5327,680\end{aligned}$ & $\begin{array}{l}\text { Nolume ellow } \\
\text { Strm.A00.A703.CRY1PRD }\end{array}$ & & 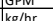 & 0.000 & $\begin{array}{l}2.30 \\
2.30\end{array}$ & 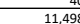 & 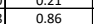 & & 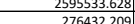 & $\frac{\$ 6,488,834}{\$ 355,799}$ \\
\hline Ccm Drier & 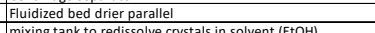 & & & 2 & 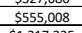 & 2011 & $\begin{array}{cccc}5327,000 \\
555,008\end{array}$ & Strm.A70.0.A7030.0RY1-PRD & 11526 & $\mathrm{~kg} / \mathrm{hr}$ & 0.60 & 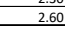 & $\frac{11,456}{10,936}$ & 0.95 & 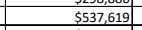 & 4972321.3374 & $\frac{5133,92,84}{\$ \$ 1,292,801}$ \\
\hline Dissolution Tank & 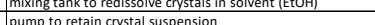 & 80 & 31655 & & $\frac{51,317,325}{563000}$ & $\frac{2011}{2009}$ & $\frac{\$ 1,317,325}{\$ 53000}$ & 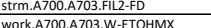 & $\frac{328984}{66}$ & kg/hr & 0.70 & $\frac{1.80}{150}$ & $\frac{54,076}{6}$ & $\frac{0.16}{100}$ & 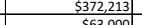 & 344250.8 & 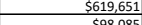 \\
\hline 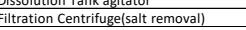 & $\begin{array}{l}\text { removes precipititated solids after dissolution } \\
\text { remons }\end{array}$ & & & & $\frac{535000}{5327,680}$ & $\frac{2009}{2011}$ & $\begin{array}{l}535,000 \\
5327,680 \\
\end{array}$ & 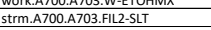 & $\frac{1303}{13403}$ & $\mathrm{~kg} / \mathrm{hr}$ & 0.60 & 2.30 & $\frac{64}{144}$ & $\frac{1.00}{0.01}$ & $\begin{array}{ll}5533,000 \\
22,074 \\
\end{array}$ & $\begin{array}{l}653300.10165 \\
20415.25834 \\
\end{array}$ & $\begin{array}{l}39,8085 \\
546,955\end{array}$ \\
\hline tDo feed tank & Iinsulated, 6460 gal & & & & 545,966 & 2011 & $\begin{aligned} 545,966 \\
5802056\end{aligned}$ & 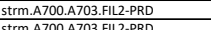 & 290932 & 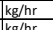 & 0.60 & 2.50 & 53,936 & 0.19 & 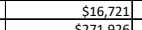 & $\begin{array}{lll}15464.71724 \\
25690.576\end{array}$ & $\begin{array}{cccc}538,662 \\
557025\end{array}$ \\
\hline $\begin{array}{l}\text { DDO reactor pump } \\
\text { HDO Feed Effluent economizer }\end{array}$ & 2-4 TEMA shell and tube $\mathrm{HX}$ & & 31655 & & $\begin{array}{l}\$ 802,861 \\
\$ 335,600\end{array}$ & $\frac{2014}{2011}$ & $\begin{array}{c}\$ 802,861 \\
\$ 3335600\end{array}$ & 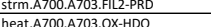 & 208726 & keghr & $\frac{0.80}{0.070}$ & $\frac{1.40}{2.66}$ & 53,936 & 0.26 & 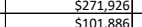 & 255689.0576 & $\frac{\$ 357,965}{\$ 520264}$ \\
\hline HoO trim preheater & 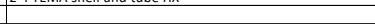 & & 30045 & & $\frac{541,000}{50}$ & 2009 & 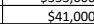 & heat:A700.A703.0H-TRIM & & MnMkcal & 0.70 & $\frac{2.260}{2.20}$ & & $\frac{0.00}{0.00}$ & 50 & & $\$ 250,2644$ \\
\hline DDO Fixed Bed Reactor & (103 FY17 milestone), base PF=2.5,208 BBL/hr & & & & 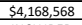 & 2011 & $\$ 44,168,568$. & Volume flow (liquid) & 328955 & L/hr & 0.70 & 2.00 & 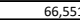 & 2.02 & 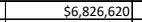 & 6313778.699 & $512,627,557$ \\
\hline $\begin{array}{l}\text { Pressure Factor (via Guthrie) } \\
\text { Interall }\end{array}$ & 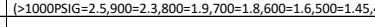 & 0:1.35) & 1.6 & & 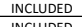 & & & 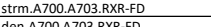 & 29274 & kg/hr & & & $53,936$. & & & & \\
\hline Hydrogenation Intercooler (bed1) & & & & & $\begin{array}{llll}52,353,181 \\
521\end{array}$ & 2007 & $\$ 2,353,181$ & heat.A700.A703.0C-BED1 & $\frac{32}{32}$ & MMlkcal & 0.65 & 2.21. & & 0.05 & 5336,676 & 367741.8853 & 5812,710 \\
\hline $\begin{array}{l}\text { Hydrogenation Intercooler (bed2) } \\
\text { Hyate }\end{array}$ & reciprocating comoressoror(5 stapes) & & & & $\frac{52,353,181}{\$ 1521200}$ & $\frac{2007}{2011}$ & $\frac{\$ 2,353,181}{\$ 51621200}$ & 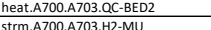 & & |l|MMkcal & 0.65 & 2.21 & & $\frac{0.09}{104}$ & $\begin{aligned} 5484,3577 \\
511661679]\end{aligned}$ & $\begin{array}{r}499384.069 \\
1536849.949\end{array}$ & 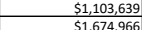 \\
\hline 12 Makeup Compressor spare & 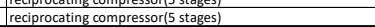 & & & & 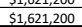 & 2011 & 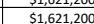 & Strm.A700.A7703.H2-MU & & kg/hrth & 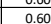 & 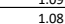 & 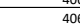 & $\frac{1.04}{1.04}$ & 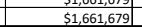 & $\begin{array}{lll}15336846.949 \\
153686.949\end{array}$ & $\frac{\$ 1,167,4666}{\$ 1,661,694}$ \\
\hline & VVia Adipic model(via MB) & & & & 5436,000 & 2013 & 5436,000 & 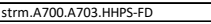 & 119841 & kg/hr & 1.00 & 1.50 & 54,336 & 0.45 & 5197,681 & 188760.8762 & 5283,141 \\
\hline $\begin{array}{l}\text { HoD hot gas cooler } \\
\text { EHPS }\end{array}$ & 3-phase horizontal sep., demister, 3/16 SS316 cladding & & & & $\frac{5321,600}{\$ 328,500}$ & $\frac{2011}{2011}$ & $\begin{array}{l}5321,600 \\
5328,500\end{array}$ & $\begin{array}{l}\text { heae.A7700 FAO703.0AC-2 } \\
\text { volum Flow }\end{array}$ & $\frac{4}{39911}$ & $\begin{array}{ll}\mid l m k k c a \\
\text { Lhr }\end{array}$ & $\frac{0.70}{0.70}$ & $\begin{array}{l}1.66 \\
2.59 \\
\end{array}$ & $\frac{9}{9}$ & 0.01 & $\begin{aligned} 51,0980 \\
5,4852\end{aligned}$ & & $\frac{\$ 16,828}{5 \$ 11,610}$ \\
\hline A evaporator feed tank & insulated, $6460 \mathrm{gal}$ & & & & & 2011 & $\frac{545,966}{5456}$ & & 290932 & $\mathrm{~kg} / \mathrm{hr}$ & 0.60 & 2.50 & $95,48$. & 0.33 & 523,557 & & 554,468 \\
\hline A evaporator feed heater & shell and tube $1 / 2$ pass & & & & $\$ 274,818$ & 2011 & 5274,818 & 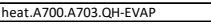 & -13 & |MMkcal & 0.60 & 3.00 & 12 & 0.12 & 577,740 & 71900.18527 & $\$ 215,701$ \\
\hline AA evapoprator flash drum & $22^{2} \times 48^{\prime}-110,000$ gal. & & 55316 & 1 & 5511,000 & 2009 & $\begin{array}{l}5511,000 \\
5\end{array}$ & 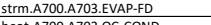 & 264116 & kg/hr & 0.70 & 2.00 & $95,48=$ & 0.36 & $\begin{array}{l}5250,676 \\
\end{array}$ & 260185.9593] & 5520,3 \\
\hline A Crrystallizer & Oslo Type. 2 In series & & 31655 & 2 series & $\frac{348,000}{57,104,192}$ & $\frac{2011}{2011}$ & $\begin{array}{rlll}\frac{3488,000}{57,104,192} \\
\end{array}$ & 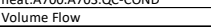 & & GPM & 0.606 & $\frac{2.80}{2.50}$ & & $\begin{array}{l}0.38 \\
0.23 \\
\end{array}$ & $\frac{25 / 2,50 \mid}{52,908,260}$ & 208069780.867 & $\frac{57,7,319}{56,4242}$ \\
\hline A Centrifuge separator & ICentrifuge Separator & & & & $\$ 327,680$ & 2011 & 5327,680 & strm-A700.A703.CRV2-PRD & 13403 & kg/hr & 0.60 & & 1118 & 0.87 & 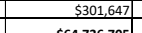 & 278985.8604 & 5641,667 \\
\hline & & & & & & & & & & & & & & Totals: & $\$ 64,736,705$ & $\$ 60,840,636$ & $\$ 128,436,573$ \\
\hline
\end{tabular}




\begin{tabular}{|c|c|c|c|c|c|c|c|c|c|c|c|c|c|c|c|c|c|}
\hline A800: CHP & Mechanical Equipment List & & & & & & & & & & dinstal & led costs & & & & & \\
\hline EQUIPMENT TITLE & DESCRIPTION & HP & MATERIAL & 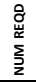 & $\$$ & 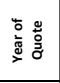 & $\left|\begin{array}{c}\text { Purch Cost in } \\
\text { Base Yr }\end{array}\right|$ & Scaling Variable & 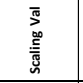 & $\frac{2}{5}$ & 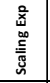 & 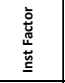 & $\begin{array}{l}\frac{\bar{j}}{3} \\
\frac{3}{2} \\
\frac{3}{2}\end{array}$ & 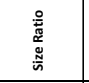 & Scaled Purch Cost & $\begin{array}{l}\text { Purch Cost in Proj } \\
\text { vear }\end{array}$ & Inst cost in Proj year \\
\hline $\begin{array}{l}\text { High Solids Burner and Turbine } \\
\text { Burner commustion Air reheater }\end{array}$ & INCLUDDED & & & & & & & & & & & & & & & & \\
\hline BFW Prehenater & INCLUDED & & & 1 & INCLUDED & & & & & & & & & & & & \\
\hline $\begin{array}{l}\text { Pertrtaatment/BFW heat recovery } \\
\text { Air nthake Fan }\end{array}$ & \begin{tabular}{|l}
$9.4 \mathrm{MM}$ Btu/hr \\
IICLUDED
\end{tabular} & & 55304 & 1 & $\begin{array}{l}\frac{541,000}{\text { INCUDED }} \\
\end{array}$ & 2009 & 541,000 & heat.A800.A810.0H812 & & Gcal/hr & 0.70 & 2.2 & (2) & 0.66 & $\$ 30,504$ & $\$ 31,662$ & 569,655 \\
\hline & 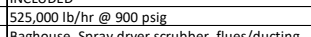 & & cs & 1 & $\frac{\$ 28,50,000}{\$ 2,150,000}$ & 2010 & $\$ 28,550,000$ & 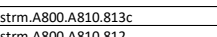 & 238203 & $\mathrm{~kg} / \mathrm{hr}$ & 0.60 & & $\begin{array}{lll}177,386 \\
113697\end{array}$ & 0.74 & $\$ 23,921,550$ & $\$ 23,526,332$ & $\begin{array}{lllll}542,347,398 & 50\end{array}$ \\
\hline $\begin{array}{l}\text { Combustion nas Baghouse } \\
\text { Turbine//enerator }\end{array}$ & $\begin{array}{l}\text { Baghouose, Spray dryer scrubber, flues/ducting } \\
23.6 \mathrm{kWW}, 2 \text { extractions }\end{array}$ & & & $\frac{1}{1}$ & $\begin{array}{l}\frac{511,0,00,000}{59,50,000} \\
\end{array}$ & $\frac{2013}{2010}$ & $\begin{aligned} 5.0 \\
59,50,000\end{aligned}$ & $\begin{array}{l}\text { Strmim.A8000.A10.812 } \\
\text { work.A900.wtotal }\end{array}$ & 238203 & $\mathrm{~kg} / \mathrm{hr}$ & $\begin{array}{c}0.60 \\
0.60 \\
\end{array}$ & $\begin{array}{c}1.8 \\
1.8 \\
\end{array}$ & $\frac{113,682]}{(11,796)}$ & 0.48 & $\begin{aligned} \$ 4,421,675 \\
\end{aligned}$ & $\begin{array}{rl}54,348,623 & 1\end{array}$ & $\begin{aligned} 50 \\
57,827,521 \\
\end{aligned}$ \\
\hline Hot Process Water Softener System & & & & 1 & $\$ 78,000$ & & $\begin{array}{r}578,000 \\
5800\end{array}$ & & 235803 & & & & & & $\begin{array}{l}550,348 \\
\end{array}$ & 549,516 & 589,128 \\
\hline $\begin{array}{ll}\text { Amine Addition Pgg } \\
\text { Ammonia Addition Pkg }\end{array}$ & & & & $\frac{1}{1}$ & $\frac{\$ 40,000}{\text { IICUDED }}$ & 2010 & $\$ 40,000$ & strm.A800.A810.812 & 235803] & $\mathrm{kg} / \mathrm{hr}$ & 0.00 & 1.8 & 113,682 & 0.48 & $\$ 40,000$ & $\$ 39,339$ & $\$ 70,810$ \\
\hline $\begin{array}{l}\text { Phosphate Addition Pkg. } \\
\text { Condensto }\end{array}$ & -1 & & & $\frac{1}{1}$ & $\begin{array}{l}\text { INCLUDED } \\
\text { INCLUDED } \\
\end{array}$ & & & & & & & & & & & & \\
\hline $\begin{array}{l}\text { Londensate Pump } \\
\text { Turbine Condensate Pump }\end{array}$ & & & $\frac{55366}{55304}$ & $\frac{2}{2}$ & $\begin{array}{l}\text { INCLDEED } \\
\text { INCLUDED } \\
\end{array}$ & & & & & & & & & & & & \\
\hline $\begin{array}{l}\text { Deaerator Feed Pump } \\
\text { BFW Pump }\end{array}$ & & & $\begin{array}{c}55304 \\
5024\end{array}$ & & & & & & & & & & & & & & \\
\hline $\begin{array}{l}\text { BEFW Pump } \\
\text { Blowdown Pump }\end{array}$ & & & 55316 & $\frac{5}{2}$ & $\begin{array}{l}\text { INCLUDED } \\
\text { ICLUEDD }\end{array}$ & & & & & & & & & & & & \\
\hline Amine Transfer Pump & & & $\frac{\mathrm{cs}}{\mathrm{cs}}$ & $\frac{2}{1}$ & $\begin{array}{ll}\text { INCLUDED } \\
\text { INCLUDED }\end{array}$ & & & & & & & & & & & & \\
\hline Condensate Collection Tank & & & A285C & 1 & $\begin{array}{l}\text { INCLUDED } \\
\text { ICWDED }\end{array}$ & & & & & & & & & & & & \\
\hline \begin{tabular}{|l} 
Condensaste Surge Drum \\
Deaerator \\
\end{tabular} & Tray type & & 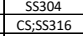 & $\frac{1}{1}$ & $\begin{array}{l}\text { IICLCLDED } \\
\$ 305,000 \\
\end{array}$ & 2010 & $\$ 305,000 \mid$ & Strm.A800.A810.812 & 235803 & kkg/hr & 0.60 & 3.0 & 113,682 & 0.48 & $\$ 196,872$ & $\$ 193,620$ & $\$ 580,859$ \\
\hline $\begin{array}{l}\text { Blowdown Flash Drum } \\
\text { Amine Drum }\end{array}$ & & & 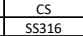 & $\frac{1}{1}$ & $\begin{array}{l}\text { INCLLDED } \\
\text { INCLODED } \\
\end{array}$ & & & & & & 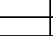 & & & & & & \\
\hline & & & & & & & & & & & & & & ea 800 Totals & $\$ 28,660,950$ & $\$ 28,189,091$ & $\$ 50,985,372$ \\
\hline A900: Utilities \& Storage & Mecha & ent List & & & & & & & & & ed instal & led costs & & & & & \\
\hline EQUIPMENT TITLE & DESCRIPTION & HP & MATERIAL & 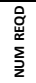 & $\$$ & 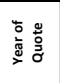 & $\mid \begin{array}{c}\text { Purch cost in } \\
\text { Base } \mathrm{Yr}\end{array}$ & Scaling Variable & 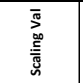 & $\frac{2}{5}$ & 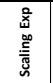 & 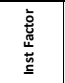 & $\begin{array}{l}\text { 嵒 } \\
\frac{3}{2}\end{array}$ & 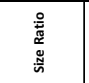 & Scaled Purch Cost & $\begin{array}{l}\text { Purch Cost in Proj } \\
\text { vear }\end{array}$ & Inst cost in Proj year \\
\hline $\begin{array}{l}\text { Utilities System } \\
\text { Soplins Tomor Sustem }\end{array}$ & & & & & & & & & & & & & & & & & \\
\hline $\begin{array}{l}\text { Cooling Tower System } \\
\text { Plant Air Compressor }\end{array}$ & $\begin{array}{l}4.4,20 \text { gom } \\
4000 \text { ScFM } 125 \text { psig }\end{array}$ & $\begin{array}{ll}750 \mathrm{Op} \\
150 \mathrm{hp}\end{array}$ & FiBRRGLASS & $\frac{1}{1}$ & $\begin{array}{l}\frac{\$ 1,375,000}{\$ 28,000} \\
\end{array}$ & $\frac{2010}{2010}$ & $\frac{\$ 1,35,000}{\$ 28,000}$ & $\begin{array}{l}\text { Strmm.a900.94s } \\
\text { DRY101 }\end{array}$ & 100378200 & kg/hr $\mathrm{k} / \mathrm{hr}$ & 0.60 & $\begin{array}{r}1.5 \\
1.6 \\
\end{array}$ & $\begin{array}{r}10,448,831 \\
8,333\end{array}$ & $\frac{1.104}{1.00}$ & $\begin{array}{ll}\$ 1,40,48,509 \\
522,000\end{array}$ & $\frac{\$ 1,385,239}{5257537}$ & 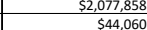 \\
\hline $\begin{array}{l}\text { Pantiled Wompressor } \\
\text { Chilled Packege }\end{array}$ & $2 \times 2350$ tons $(14.2 \mathrm{MM} \mathrm{kcal} / \mathrm{hr})$ & $\begin{array}{ll}\frac{150 \mathrm{hp}}{3400 \mathrm{hp}} \\
3\end{array}$ & & $\frac{1}{1}$ & $\begin{array}{l}\$ 2,8,000 \\
\$ 1,27,750 \\
\end{array}$ & 2010 & $\$ 1,275,750$ & heat.a900.qchwop & $\frac{83353}{14}$ & $\frac{\mathrm{kgghr}}{\mathrm{Gcal/hr}}$ & 0.60 & $\frac{1.6}{1.6}$ & $\begin{array}{r}83,333 \\
51 \\
\end{array}$ & $\frac{1.00}{3.60}$ & $\frac{528,000}{52,799,53}$ & 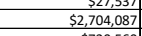 & $\$ 44,326,5390$ \\
\hline $\begin{array}{l}\text { CPIP System } \\
\text { Cooling Water Pump }\end{array}$ & 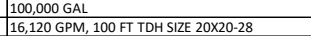 & 500.0 & $\frac{55304 / 5531}{c s}$ & $\frac{1}{3}$ & $\begin{array}{l}\$ 421,000 \\
\$ 283,671 \\
\end{array}$ & $\frac{2009}{2010}$ & $\begin{aligned} \$ 421,000 \\
\$ \$ 283,671 \\
\end{aligned}$ & $\begin{array}{l}\text { strtr.a9000.914 } \\
\text { strm.a900.945 }\end{array}$ & $\frac{63}{10982556}$ & kg/hr $k$ khr & 0.600 & $\frac{1.8}{3 .}$ & $\begin{aligned} 1455 \\
10,448,831 \\
\end{aligned}$ & $\frac{2.30}{0.95}$ & $\begin{array}{l}5594,222 \\
\$ 2272,588\end{array}$ & $\begin{array}{l}\$ \$ 720,500 \\
\$ 268,084\end{array}$ & 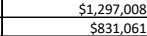 \\
\hline 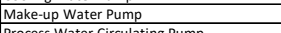 & 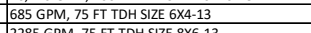 & 20.0 & cs & 1 & 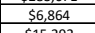 & 2010 & 56.864 & 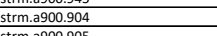 & 155564 & $\mathrm{~kg} / \mathrm{hr}$ & 0.80 & $\frac{3.1}{3.1}-10 \mathrm{ll}$ & $\begin{aligned} 2,440,051 \\
445,086 \\
\end{aligned}$ & 2.86 & $\begin{array}{ll}5 \\
515,915 \\
\end{array}$ & $\begin{array}{ll}3200304 \\
515,652 \\
\end{array}$ & $\frac{5051,01}{548,521}$ \\
\hline $\begin{array}{l}\text { Process Water Circulating Pump } \\
\text { Instrument Air Dryer }\end{array}$ & 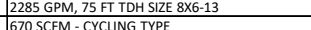 & 75.0 & cs & 1 & $\frac{\$ 15,292}{\$ \$ 51500}$ & $\frac{2010}{2009}$ & $\begin{array}{l}\$ 15,292 \\
\$ 15,0001 \\
\end{array}$ & $\begin{array}{l}\text { Strm..9900.905 } \\
\text { DVY101 }\end{array}$ & 518924 & kg/hr & 0.80 & 3.1 & $445,086]$ & 0.86 & $\begin{array}{c}\$ 13,525 \\
\$ 15000 \\
\end{array}$ & \begin{tabular}{c|c|}
$\$ 13,302$ \\
$\$ 15,569$
\end{tabular} & $\frac{54,235}{5.2025}$ \\
\hline $\begin{array}{l}\text { nntrtument Air Dryer } \\
\text { Plant Air Receiver }\end{array}$ & 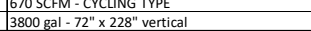 & & $\frac{c s}{c s}$ & $\frac{1}{1}$ & $\frac{\$ 515,00}{\$ 16,000}$ & $\frac{2009}{2009}$ & $\frac{\$ 15,000 \mid}{\$ 16,000}$ & $\begin{array}{l}\text { DRY1011 } \\
\text { DRY101 }\end{array}$ & 833333 & $\frac{k g / h r}{k g / h r}$ & $\begin{array}{c}0.60 \\
.600 \\
\end{array}$ & $\frac{1.8}{3.1}$ & $\begin{aligned} 83,3333 \\
8333\end{aligned}$ & $\frac{1.100}{1.00}$ & $\frac{\$ 15,000}{\$ \$ 16,000}$ & $\frac{\$ 515,59}{\$ 15,607}$ & $\frac{528,024}{551828}$ \\
\hline Process Water Tank No. 1 & $250,000 \mathrm{gal}$ & & cs & 1 & $\begin{array}{ll}5250,000 \\
52500\end{array}$ & 2009 & $\$ 250,000$ & strm.a900.905 & 451555 & $\mathrm{~kg} / \mathrm{hr}$ & 0.70 & & 445,0356 & 0.99 & $\$ 247,488$ & $\begin{array}{ccc}\$ 520,077 \\
\$ 2577\end{array}$ & 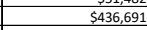 \\
\hline $\begin{array}{l}\text { Storage } \\
\text { Ammonia Storage Tank }\end{array}$ & $28,000 \mathrm{gal}$ & & $54-51670,070$ & & $\$ 196000$ & & & CTM AOOONHZNET & - & & & & & & & 5196398 & \\
\hline cSLL Storagege Tank ank & 70,000 gal & & $\begin{array}{lll}S A S B D T \\
\text { Glass lined }\end{array}$ & $\frac{2}{1}$ & $\begin{array}{l}\frac{3190,000}{570,000} \\
\end{array}$ & $\frac{2010}{2009}$ & 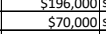 & Strm.A900.CSLLNET & $\begin{array}{l}\frac{11711}{1393} \\
\end{array}$ & kg/hr hr & 0.70 & & $\begin{aligned} 1,203 \\
800 \\
\end{aligned}$ & $\frac{1.03}{0.57}$ & $\frac{5199,697 / 7}{547,483}$ & 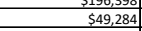 & 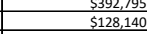 \\
\hline $\begin{array}{l}\text { CSL L Storage Tank Agitator } \\
\text { SSL }\end{array}$ & & $10 \mathrm{hp}$ & 55304 & 1 & $\$ 21,200$ & 2009 & $\$ 21,200 \mid$ & Strm.A900.CSL-NET & 1393 & $\mathrm{~kg} / \mathrm{hr}$ & 0.50 & 1.5 & 800 & 0.57 & $\begin{array}{l}\$ 16,067 \\
1,07\end{array}$ & $\$ 16,677$ & $\$ 25,015$ \\
\hline $\begin{array}{l}\text { SCL L Pump Bulk Bag Unloader } \\
\text { DAP }\end{array}$ & $\begin{array}{l}8 \text { SGPM, } 80 \mathrm{FF} \text { T TDH } \\
\text { Super sack unloader }\end{array}$ & 0.5 & cs & $\frac{1}{1}$ & $\frac{\$ 3,000}{\$ 30,000}$ & $\frac{2009}{2009}$ & $\begin{aligned} \$ 3,000 \\
33,000 \\
\end{aligned}$ & 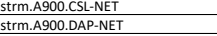 & $\begin{array}{r}1393 \mid \\
163 \\
\end{array}$ & kg/hr $k \mathrm{hr}$ & 0.800 & $\begin{array}{l}3.1 \\
1.7 \\
\end{array}$ & $\begin{array}{r}8000 \\
627 \\
\end{array}$ & $\frac{0.57}{3.84}$ & $\begin{aligned} 1,925 \mid \\
567,301 \\
\end{aligned}$ & $\begin{aligned} 51,998 \\
\$ 56,854 \\
\end{aligned}$ & $\begin{aligned} 56,195 \\
\$ 118,753 \\
\end{aligned}$ \\
\hline AAP Bulk Bag Holder & Super sack holder & & & 1 & INCLIUDED & & & & & & & & & & & & \\
\hline DAP Make-up Tank & $12,800 \mathrm{gal}$ & & 55304 & 1 & $\$ 102,000$ & 2009 & $\$ 102,000 \mid$ & strm.A900.DAP-NET & 1615 & $\mathrm{~kg} / \mathrm{hr}$ & 0.70 & 1.8 & 627 & 0.39 & $\$ 552,577$ & $\$ 54,572$ & $\$ 99,2299$ \\
\hline DAP Make-up Tank Agitator & & $5.5 \mathrm{hp}$ & 55304 & 1 & 59,800 & 2009 & $\$ 9,800 \mid$ & Strm.A900.DAP-NET & & kg/hr & 0.50 & 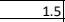 & 627 & 3.84 & 519,215 & $\$ 19,944$ & $\$ 29,916$ \\
\hline DAP Pump & 2 GPM, 100 FT TDH & 0.5 & $\frac{c s}{c s 16}$ & 1 & $\$ 3,000$ & 2009 & $53,000 \mid$ & Strm.A900.DAP-NET & 163 & kg/hr & 0.80 & 3.1 & 627 & 3.84 & 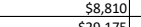 & 59,145 & 528,348 \\
\hline $\begin{array}{l}\text { Sulfuric Adcid Pump } \\
\text { Sulturic Adid Storage Tank }\end{array}$ & 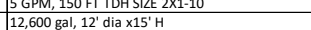 & 0.5 & $\frac{55316}{55}$ & $\frac{1}{1}$ & $\begin{array}{l}\$, 793 \\
\$ 96,000 \\
\end{array}$ & $\frac{2010}{2010}$ & $\begin{array}{ccc}57,4933 \\
5900003\end{array}$ & $\begin{array}{l}\text { strm.AA00.ACIDDNET } \\
\text { strm.A900.ACID-NET }\end{array}$ & $\frac{1981}{1981}$ & $\mathrm{kgghr} / \mathrm{hr}$ & 0.80 & 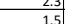 & $\frac{10,835}{10835}$ & 5.47 & $\begin{array}{c}\$ 29,175 \\
\$ 35,376\end{array}$ & $\frac{528,693}{\$ 330105}$ & 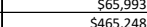 \\
\hline 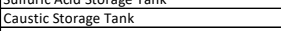 & 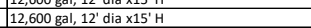 & & $\frac{53}{5 s}$ & 1 & $\begin{array}{l}\frac{396,0,00}{596,000} \\
\$(1)\end{array}$ & 2011 & $\begin{array}{ll}596,000 \\
96,000\end{array}$ & Strm.A900. BASE-NET & 1981 & $\mathrm{kg} / \mathrm{hr}$ & 0.70 & 1.5 & 8,894 & $\begin{array}{r}5.47 \\
4.29 \\
\end{array}$ & 5265.9699 & 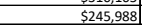 & $\begin{aligned} 560,4,48 \\
368,982 \\
\end{aligned}$ \\
\hline Firewater Storage Tank & & & Glass lined & 1 & 5803,000 & 2009 & $\$ 803,000$ & Strm.A900.H2O-FIRE & 8843 & $\frac{1 \mathrm{~kg} / \mathrm{hr}}{\mathrm{s}}$ & 0.70 & $1.7 \mathrm{r}, \mathrm{C}, \mathrm{C}$ & 8.343 & 1.00 & 5803,000 & $5833,464 \mid$ & $\$ 1,416,890$ \\
\hline $\begin{array}{l}\text { Firewater Pump } \\
\text { Rimate pun }\end{array}$ & 2500 GPM, 150 FT TOH & 125.0 & cs & 1 & 515,000 & 2009 & $\$ 15,000$ & $\begin{array}{l}\text { strm.A900.H2O-FIRE } \\
\text { trm.500 }\end{array}$ & 8343 & $\mathrm{~kg} / \mathrm{hr}$ & 0.80 & 3.11 & 8,343 & 1.00 & $\$ 15,000$ & S15,569] & 548,264 \\
\hline $\begin{array}{l}\text { Diesele tsoraget entk } \\
\text { co-Product Storage Tank(Adipic) }\end{array}$ & 750,000 gall, 7 day storage, , flating roof & & A285C & $\frac{1}{1}$ & $\begin{array}{l}\frac{5677,000}{5600,900} \\
5600\end{array}$ & $\frac{2009}{2007}$ & $\begin{aligned} \$ \$ 670,000 \\
5600,900 \\
\end{aligned}$ & & $\frac{113411}{2332290249}$ & $\mathrm{~kg} / \mathrm{hr} \mathrm{hr}$ & 0.70 & $\frac{1.7}{1.50}$ & $\frac{-11,032}{1111211}$ & $\frac{0.97}{0.077823101}$ & $\frac{\$ 557,171}{5426,926}$ & $\frac{\$ 682,103}{\$ 440171}$ & $\frac{\$ 1,159,575}{\$ \$ 14317}$ \\
\hline 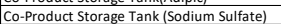 & & & & $\frac{1}{1}$ & $\begin{array}{l}\frac{5609,900}{560,900} \\
5600\end{array}$ & 2007 & 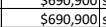 & Strm.PRDD- -600 & 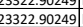 & $\mathrm{kg} / \mathrm{hr}$ & $\begin{array}{l}0.65 \\
0.65 \\
\end{array}$ & $\begin{array}{l}1.880 \\
.850 \\
\end{array}$ & $\begin{array}{ll}1,1121 \\
14,163\end{array}$ & 0.0 .60725557778 & $\begin{aligned} 5462,926 \\
5499,582\end{aligned}$ & 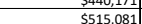 & $\begin{array}{l}5884,311 \\
5952,900\end{array}$ \\
\hline $\begin{array}{l}\text { Glucose Storage Tank } \\
\end{array}$ & $\begin{array}{ll}70,000 \text { gal } \\
\text { gal }\end{array}$ & & $\begin{array}{ll}\text { Glass lined } \\
\end{array}$ & 1 & 570,000 & 2009 & & strm.a400.401 & & & 0.70 & & & 1.12 & 575,686 & 578,557 & $\$ 204,249$ \\
\hline & & & & & & & & & & & & & & ea 900 Totals & $\$ 8,961,720$ & $\$ 8,991,177$ & $\$ 15,506,286$ \\
\hline
\end{tabular}




\title{
Appendix B. Discounted Cash Flow Rate of Return Worksheet
}

\author{
Acids Pathway
}

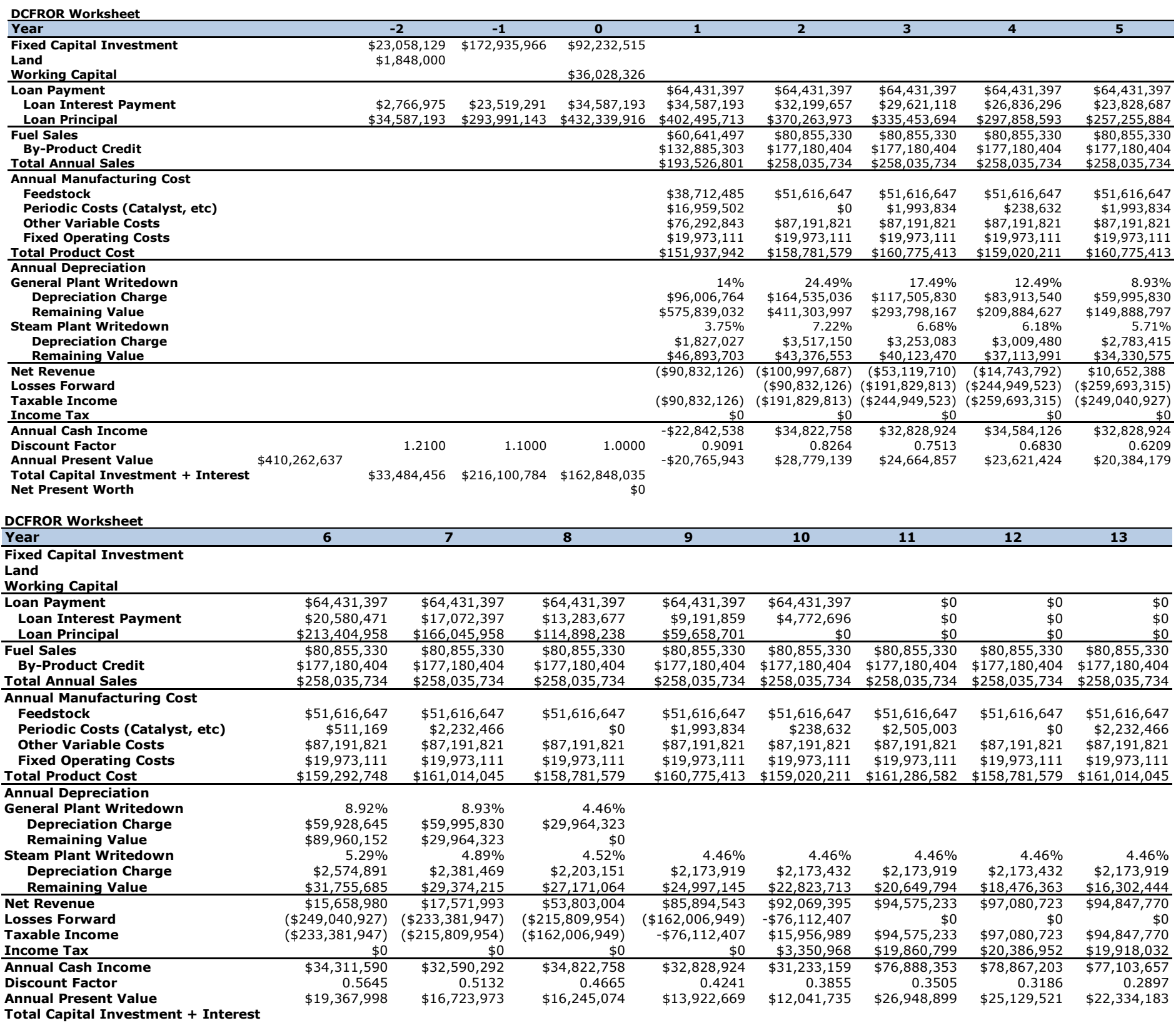

Total Capital Investment + Interest

Net Present Worth 
DCFROR Worksheet

\begin{tabular}{|c|c|c|c|c|c|c|c|c|}
\hline Year & 14 & 15 & 16 & 17 & 18 & 19 & 20 & 21 \\
\hline \multicolumn{9}{|l|}{$\begin{array}{l}\text { Fixed Capital Investment } \\
\text { Land } \\
\text { Working Capital }\end{array}$} \\
\hline Loan Payment & $\$ 0$ & $\$ 0$ & $\$ 0$ & $\$ 0$ & $\$ 0$ & $\$ 0$ & $\$ 0$ & $\$ 0$ \\
\hline Loan Interest Payment & $\$ 0$ & $\$ 0$ & $\$ 0$ & $\$ 0$ & $\$ 0$ & $\$ 0$ & $\$ 0$ & $\$ 0$ \\
\hline Loan Principal & $\$ 0$ & $\$ 0$ & $\$ 0$ & $\$ 0$ & $\$ 0$ & $\$ 0$ & $\$ 0$ & $\$ 0$ \\
\hline Fuel Sales & $\$ 80,855,330$ & $\$ 80,855,330$ & $\$ 80,855,330$ & $\$ 80,855,330$ & $\$ 80,855,330$ & $\$ 80,855,330$ & $\$ 80,855,330$ & $\$ 80,855,330$ \\
\hline By-Product Credit & $\$ 177,180,404$ & $\$ 177,180,404$ & $\$ 177,180,404$ & $\$ 177,180,404$ & $\$ 177,180,404$ & $\$ 177,180,404$ & $\$ 177,180,404$ & $\$ 177,180,404$ \\
\hline Total Annual Sales & $\$ 258,035,734$ & $\$ 258,035,734$ & $\$ 258,035,734$ & $\$ 258,035,734$ & $\$ 258,035,734$ & $\$ 258,035,734$ & $\$ 258,035,734$ & $\$ 258,035,734$ \\
\hline \multicolumn{9}{|l|}{ Annual Manufacturing Cost } \\
\hline Feedstock & $\$ 51,616,647$ & $\$ 51,616,647$ & $\$ 51,616,647$ & $\$ 51,616,647$ & $\$ 51,616,647$ & $\$ 51,616,647$ & $\$ 51,616,647$ & $\$ 51,616,647$ \\
\hline Periodic Costs (Catalyst, etc) & $\$ 0$ & $\$ 1,993,834$ & $\$ 749,801$ & $\$ 1,993,834$ & $\$ 0$ & $\$ 2,232,466$ & $\$ 0$ & $\$ 2,505,003$ \\
\hline Other Variable Costs & $\$ 87,191,821$ & $\$ 87,191,821$ & $\$ 87,191,821$ & $\$ 87,191,821$ & $\$ 87,191,821$ & $\$ 87,191,821$ & $\$ 87,191,821$ & $\$ 87,191,821$ \\
\hline Fixed Operating Costs & $\$ 19,973,111$ & $\$ 19,973,111$ & $\$ 19,973,111$ & $\$ 19,973,111$ & $\$ 19,973,111$ & $\$ 19,973,111$ & $\$ 19,973,111$ & $\$ 19,973,111$ \\
\hline Total Product Cost & $\$ 158,781,579$ & $\$ 160,775,413$ & $\$ 159,531,380$ & $\$ 160,775,413$ & $\$ 158,781,579$ & $\$ 161,014,045$ & $\$ 158,781,579$ & $\$ 161,286,582$ \\
\hline
\end{tabular}

\section{Annual Depreciation}

General Plant Writedown

Depreciation Charge

Remaining Value

Steam Plant

Depreciation Charge

Remaining Value

Net Revenue

Losses Forward

Taxable Income

Income Tax

Annual Cash Income

Discount Factor

Annual Present Value

$158,781,579 \$ 160,775,413$

\begin{tabular}{llllll}
$\$ 159,531,380$ & $\$ 160,775,413$ & $\$ 158,781,579$ & $\$ 161,014,045$ & $\$ 158,781,579$ & $\$ 161,286,582$ \\
\hline
\end{tabular}

Total Capital Investment + Interest

Net Present Worth

\begin{tabular}{|c|c|c|c|c|c|c|c|c|c|}
\hline Year & 22 & 23 & 24 & 25 & 26 & 27 & 28 & 29 & 30 \\
\hline $\begin{array}{l}\text { Fixed Capital Investment } \\
\text { Land } \\
\text { Working Capital }\end{array}$ & & & & & & & & & $\begin{array}{r}(\$ 1,848,000) \\
(\$ 36,028,326)\end{array}$ \\
\hline Loan Payment & $\$ 0$ & $\$ 0$ & $\$ 0$ & $\$ 0$ & $\$ 0$ & $\$ 0$ & $\$ 0$ & $\$ 0$ & $\$ 0$ \\
\hline Loan Interest Payment & $\$ 0$ & $\$ 0$ & $\$ 0$ & $\$ 0$ & $\$ 0$ & $\$ 0$ & $\$ 0$ & $\$ 0$ & $\$ 0$ \\
\hline Loan Principal & $\$ 0$ & $\$ 0$ & $\$ 0$ & $\$ 0$ & $\$ 0$ & $\$ 0$ & $\$ 0$ & $\$ 0$ & $\$ 0$ \\
\hline Fuel Sales & $\$ 80,855,330$ & $\$ 80,855,330$ & $\$ 80,855,330$ & $\$ 80,855,330$ & $\$ 80,855,330$ & $\$ 80,855,330$ & $\$ 80,855,330$ & $\$ 80,855,330$ & $\$ 80,855,330$ \\
\hline By-Product Credit & $\$ 177,180,404$ & $\$ 177,180,404$ & $\$ 177,180,404$ & $\$ 177,180,404$ & $\$ 177,180,404$ & $\$ 177,180,404$ & $\$ 177,180,404$ & $\$ 177,180,404$ & $\$ 177,180,404$ \\
\hline Total Annual Sales & $\$ 258,035,734$ & $\$ 258,035,734$ & $\$ 258,035,734$ & $\$ 258,035,734$ & $\$ 258,035,734$ & $\$ 258,035,734$ & $\$ 258,035,734$ & $\$ 258,035,734$ & $\$ 258,035,734$ \\
\hline \multicolumn{10}{|l|}{ Annual Manufacturing Cost } \\
\hline Feedstock & $\$ 51,616,647$ & $\$ 51,616,647$ & $\$ 51,616,647$ & $\$ 51,616,647$ & $\$ 51,616,647$ & $\$ 51,616,647$ & $\$ 51,616,647$ & $\$ 51,616,647$ & $\$ 51,616,647$ \\
\hline Periodic Costs (Catalyst, etc) & $\$ 238,632$ & $\$ 1,993,834$ & $\$ 0$ & $\$ 2,232,466$ & $\$ 511,169$ & $\$ 1,993,834$ & $\$ 238,632$ & $\$ 1,993,834$ & $\$ 0$ \\
\hline Other Variable Costs & $\$ 87,191,821$ & $\$ 87,191,821$ & $\$ 87,191,821$ & $\$ 87,191,821$ & $\$ 87,191,821$ & $\$ 87,191,821$ & $\$ 87,191,821$ & $\$ 87,191,821$ & $\$ 87,191,821$ \\
\hline Fixed Operating Costs & $\$ 19,973,111$ & $\$ 19,973,111$ & $\$ 19,973,111$ & $\$ 19,973,111$ & $\$ 19,973,111$ & $\$ 19,973,111$ & $\$ 19,973,111$ & $\$ 19,973,111$ & $\$ 19,973,111$ \\
\hline Total Product Cost & $\$ 159,020,211$ & $\$ 160,775,413$ & $\$ 158,781,579$ & $\$ 161,014,045$ & $\$ 159,292,748$ & $\$ 160,775,413$ & $\$ 159,020,211$ & $\$ 160,775,413$ & $\$ 158,781,579$ \\
\hline \multicolumn{10}{|l|}{$\begin{array}{l}\text { Annual Depreciation } \\
\text { General Plant Writedown } \\
\text { Depreciation Charge } \\
\text { Remaining Value } \\
\text { Steam Plant Writedown } \\
\text { Depreciation Charge } \\
\text { Remaining Value }\end{array}$} \\
\hline Net Revenue & $\$ 99,015,523$ & $\$ 97,260,321$ & $\$ 99,254,155$ & $\$ 97,021,689$ & $\$ 98,742,986$ & $\$ 97,260,321$ & $\$ 99,015,523$ & $\$ 97,260,321$ & $\$ 99,254,155$ \\
\hline Losses Forward & $\$ 0$ & $\$ 0$ & $\$ 0$ & $\$ 0$ & $\$ 0$ & $\$ 0$ & $\$ 0$ & $\$ 0$ & $\$ 0$ \\
\hline Taxable Income & $\$ 99,015,523$ & $\$ 97,260,321$ & $\$ 99,254,155$ & $\$ 97,021,689$ & $\$ 98,742,986$ & $\$ 97,260,321$ & $\$ 99,015,523$ & $\$ 97,260,321$ & $\$ 99,254,155$ \\
\hline Income Tax & $\$ 20,793,260$ & $\$ 20,424,667$ & $\$ 20,843,373$ & $\$ 20,374,555$ & $\$ 20,736,027$ & $\$ 20,424,667$ & $\$ 20,793,260$ & $\$ 20,424,667$ & $\$ 20,843,373$ \\
\hline Annual Cash Income & $\$ 78,222,263$ & $\$ 76,835,653$ & $\$ 78,410,782$ & $\$ 76,647,134$ & $\$ 78,006,959$ & $\$ 76,835,653$ & $\$ 78,222,263$ & $\$ 76,835,653$ & $\$ 78,410,782$ \\
\hline Discount Factor & 0.1228 & 0.1117 & 0.1015 & 0.0923 & 0.0839 & 0.0763 & 0.0693 & 0.0630 & 0.0573 \\
\hline $\begin{array}{l}\text { Annual Present Value } \\
\text { Total Capital Investment + Interest }\end{array}$ & $\$ 9,609,290$ & $\$ 8,580,864$ & $\$ 7,960,702$ & $\$ 7,074,224$ & $\$ 6,545,209$ & $\$ 5,860,846$ & $\$ 5,424,194$ & $\$ 4,843,674$ & $\begin{array}{r}\$ 4,493,609 \\
(\$ 2,170,637)\end{array}$ \\
\hline
\end{tabular}




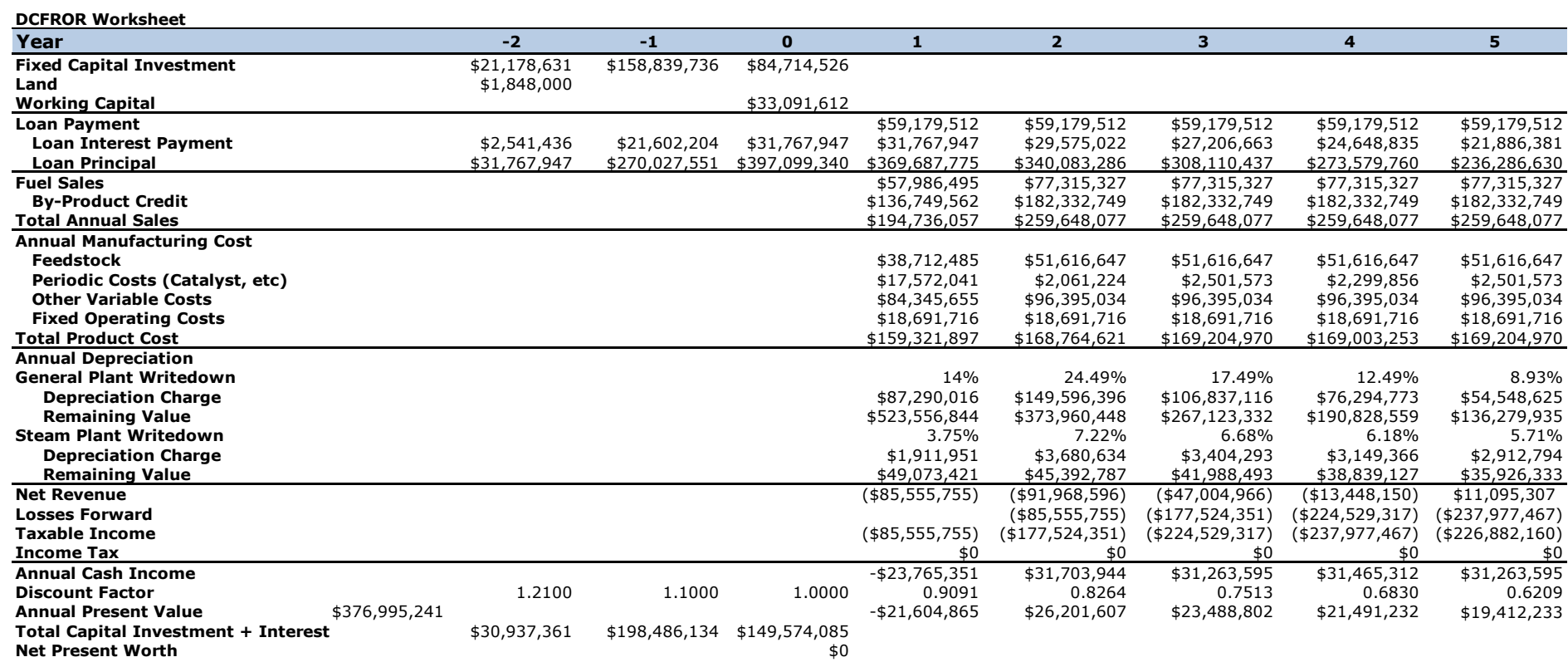

\begin{tabular}{|c|c|c|c|c|c|c|c|c|}
\hline Year & 6 & 7 & 8 & 9 & 10 & 11 & 12 & 13 \\
\hline \multicolumn{9}{|l|}{$\begin{array}{l}\text { Fixed Capital Investment } \\
\text { Land } \\
\text { Working Capital }\end{array}$} \\
\hline Loan Payment & $\$ 59,179,512$ & $\$ 59,179,512$ & $\$ 59,179,512$ & $\$ 59,179,512$ & $\$ 59,179,512$ & $\$ 0$ & $\$ 0$ & $\$ 0$ \\
\hline Loan Interest Payment & $\$ 18,902,930$ & $\$ 15,680,804$ & $\$ 12,200,907$ & $\$ 8,442,619$ & $\$ 4,383,668$ & $\$ 0$ & $\$ 0$ & $\$ 0$ \\
\hline Loan Principal & $\$ 196,010,049$ & $\$ 152,511,341$ & $\$ 105,532,737$ & $\$ 54,795,844$ & $\$ 0$ & $\$ 0$ & $\$ 0$ & $\$ 0$ \\
\hline Fuel Sales & $\$ 77,315,327$ & $\$ 77,315,327$ & $\$ 77,315,327$ & $\$ 77,315,327$ & $\$ 77,315,327$ & $\$ 77,315,327$ & $\$ 77,315,327$ & $\$ 77,315,327$ \\
\hline By-Product Credit & $\$ 182,332,749$ & $\$ 182,332,749$ & $\$ 182,332,749$ & $\$ 182,332,749$ & $\$ 182,332,749$ & $\$ 182,332,749$ & $\$ 182,332,749$ & $\$ 182,332,749$ \\
\hline Total Annual Sales & $\$ 259,648,077$ & $\$ 259,648,077$ & $\$ 259,648,077$ & $\$ 259,648,077$ & $\$ 259,648,077$ & $\$ 259,648,077$ & $\$ 259,648,077$ & $\$ 259,648,077$ \\
\hline \multicolumn{9}{|l|}{ Annual Manufacturing Cost } \\
\hline Feedstock & $\$ 51,616,647$ & $\$ 51,616,647$ & $\$ 51,616,647$ & $\$ 51,616,647$ & $\$ 51,616,647$ & $\$ 51,616,647$ & $\$ 51,616,647$ & $\$ 51,616,647$ \\
\hline Periodic Costs (Catalyst, etc) & $\$ 2,572,392$ & $\$ 2,740,205$ & $\$ 2,061,224$ & $\$ 2,501,573$ & $\$ 2,299,856$ & $\$ 3,012,742$ & $\$ 2,061,224$ & $\$ 2,740,205$ \\
\hline Other Variable Costs & $\$ 96,395,034$ & $\$ 96,395,034$ & $\$ 96,395,034$ & $\$ 96,395,034$ & $\$ 96,395,034$ & $\$ 96,395,034$ & $\$ 96,395,034$ & $\$ 96,395,034$ \\
\hline Fixed Operating Costs & $\$ 18,691,716$ & $\$ 18,691,716$ & $\$ 18,691,716$ & $\$ 18,691,716$ & $\$ 18,691,716$ & $\$ 18,691,716$ & $\$ 18,691,716$ & $\$ 18,691,716$ \\
\hline Total Product Cost & $\$ 169,275,789$ & $\$ 169,443,602$ & $\$ 168,764,621$ & $\$ 169,204,970$ & $\$ 169,003,253$ & $\$ 169,716,139$ & $\$ 168,764,621$ & $\$ 169,443,602$ \\
\hline \multicolumn{9}{|l|}{ Annual Depreciation } \\
\hline General Plant Writedown & $8.92 \%$ & $8.93 \%$ & $4.46 \%$ & & & & & \\
\hline Depreciation Charge & $\$ 54,487,540$ & $\$ 54,548,625$ & $\$ 27,243,770$ & & & & & \\
\hline Remaining Value & $\$ 81,792,395$ & $\$ 27,243,770$ & $\$ 0$ & & & & & \\
\hline Steam Plant Writedown & $5.29 \%$ & $4.89 \%$ & $4.52 \%$ & $4.46 \%$ & $4.46 \%$ & $4.46 \%$ & $4.46 \%$ & $4.46 \%$ \\
\hline Depreciation Charge & $\$ 2,694,577$ & $\$ 2,492,165$ & $\$ 2,305,559$ & $\$ 2,274,967$ & $\$ 2,274,457$ & $\$ 2,274,967$ & $\$ 2,274,457$ & $\$ 2,274,967$ \\
\hline Remaining Value & $\$ 33,231,756$ & $\$ 30,739,591$ & $\$ 28,434,032$ & $\$ 26,159,065$ & $\$ 23,884,607$ & $\$ 21,609,640$ & $\$ 19,335,183$ & $\$ 17,060,215$ \\
\hline Net Revenue & $\$ 14,287,240$ & $\$ 17,482,881$ & $\$ 49,133,220$ & $\$ 79,725,520$ & $\$ 83,986,699$ & $\$ 87,656,970$ & $\$ 88,608,998$ & $\$ 87,929,507$ \\
\hline Losses Forward & $(\$ 226,882,160)$ & $(\$ 212,594,920)$ & $(\$ 195,112,040)$ & $(\$ 145,978,820)$ & $-\$ 66,253,299$ & $\$ 0$ & $\$ 0$ & $\$ 0$ \\
\hline Taxable Income & $(\$ 212,594,920)$ & $(\$ 195,112,040)$ & $(\$ 145,978,820)$ & $-\$ 66,253,299$ & $\$ 17,733,399$ & $\$ 87,656,970$ & $\$ 88,608,998$ & $\$ 87,929,507$ \\
\hline Income Tax & $\$ 0$ & $\$ 0$ & $\$ 0$ & $\$ 0$ & $\$ 3,724,014$ & $\$ 18,407,964$ & $\$ 18,607,890$ & $\$ 18,465,196$ \\
\hline Annual Cash Income & $\$ 31,192,776$ & $\$ 31,024,963$ & $\$ 31,703,944$ & $\$ 31,263,595$ & $\$ 27,741,298$ & $\$ 71,523,974$ & $\$ 72,275,566$ & $\$ 71,739,278$ \\
\hline Discount Factor & 0.5645 & 0.5132 & 0.4665 & 0.4241 & 0.3855 & 0.3505 & 0.3186 & 0.2897 \\
\hline $\begin{array}{l}\text { Annual Present Value } \\
\text { total Capital Investment }\end{array}$ & $\$ 17,607,509$ & $\$ 15,920,712$ & $\$ 14,790,124$ & $\$ 13,258,816$ & $\$ 10,695,471$ & $\$ 25,068,717$ & $\$ 23,029,223$ & $\$ 20,780,313$ \\
\hline
\end{tabular}


DCFROR Worksheet

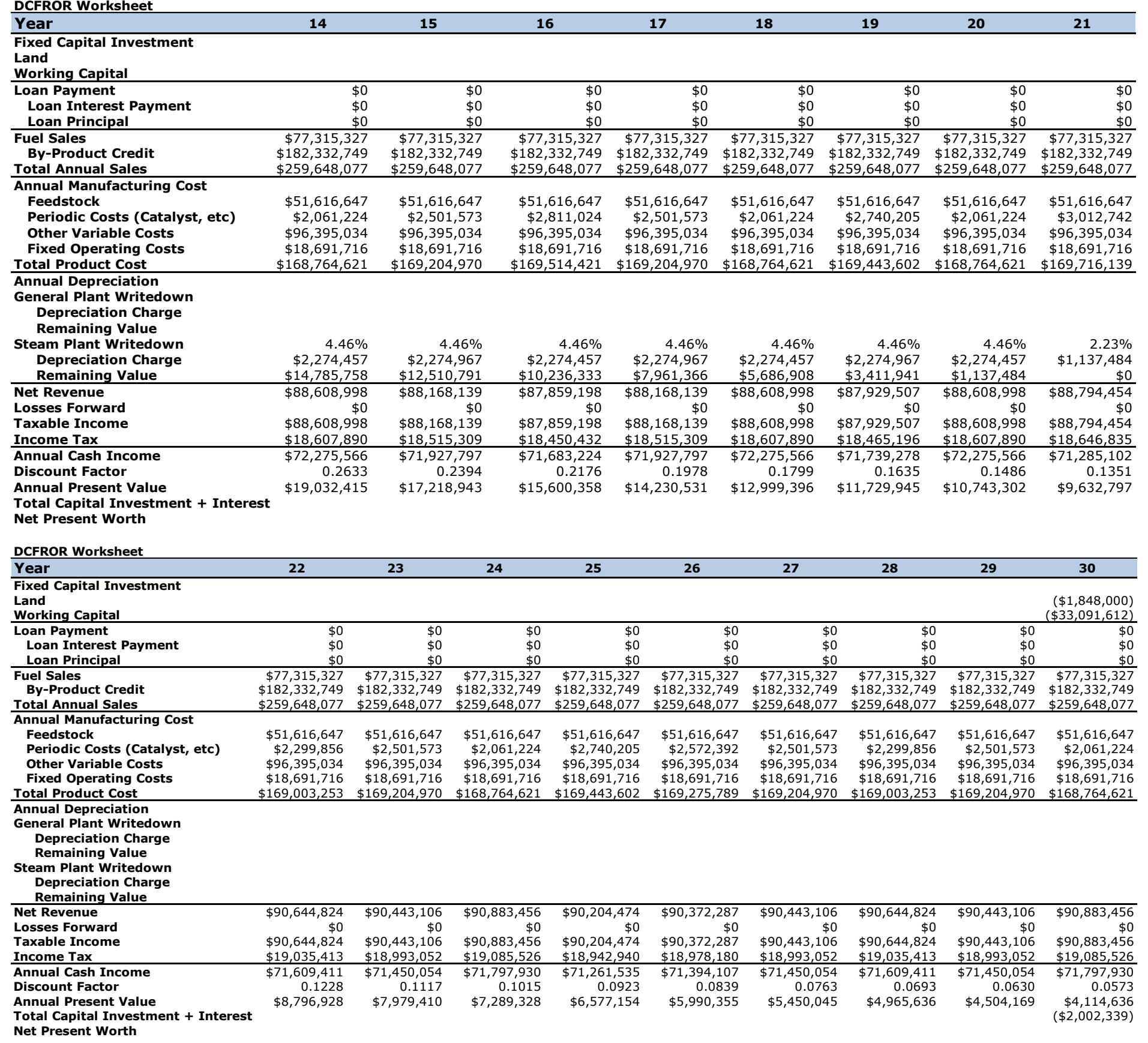




\section{Appendix C. Process Parameters/Operating Summary}

\section{Acids Pathway}

\begin{tabular}{|lr|}
\hline Final Fuel Product & \\
\hline Fuel Mass flow (kg/hr) & 11465.5 \\
Estimated Density (kg/L) & 0.6 \\
Fuel Volume Flow (L/hr) & 18792.0 \\
Fuel LHV (Mmkcal/hr) & 120.5 \\
Annual Fuel Volume (MM Gal/yr) & 39.1 \\
Annual Fuel GGE (MM GGE/yr) & 32.5 \\
\hline
\end{tabular}

\begin{tabular}{|c|c|}
\hline \multicolumn{2}{|l|}{ A100: Feedstock } \\
\hline Moisture Content & $20 \%$ \\
\hline \multicolumn{2}{|l|}{ Composition (wt\% Dry Feed) } \\
\hline Cellulose & $35.0 \%$ \\
\hline Xylan & $19.5 \%$ \\
\hline Arabinan & $2.4 \%$ \\
\hline Sucrose & $0.8 \%$ \\
\hline Lignin & $15.8 \%$ \\
\hline Acetate & $1.8 \%$ \\
\hline Extract & $14.6 \%$ \\
\hline Ash & $4.9 \%$ \\
\hline Mannan & $0.6 \%$ \\
\hline Galactan & $1.4 \%$ \\
\hline Protein & $3.1 \%$ \\
\hline \multicolumn{2}{|c|}{ Hydrolysis Mass yield (g/g biopolymer) } \\
\hline Cellulose to glucose & 1.111109 \\
\hline Xylan to xylose & 1.136361 \\
\hline Arabinan to arabinose & 1.136361 \\
\hline \multicolumn{2}{|l|}{ A200: Deacetylation \& Pretreatment } \\
\hline \multicolumn{2}{|l|}{ Deacetylation Conditions } \\
\hline Temperature (C) & 90 \\
\hline Pressure (atm) & 1 \\
\hline $\mathrm{NaOH}$ loading (mg/L) & 70 \\
\hline \multicolumn{2}{|l|}{ Solubilization to the Black Liquor } \\
\hline Acetate & $100 \%$ \\
\hline Lignin & $47.0 \%$ \\
\hline Cellulose & $2.0 \%$ \\
\hline Xylan & $10.0 \%$ \\
\hline Arabinan & $30.0 \%$ \\
\hline \multicolumn{2}{|c|}{ A300: Continuous Enzymatic Hydrolysis } \\
\hline \multicolumn{2}{|c|}{ Operating Conditiond } \\
\hline Enzyme Loading & 9.96 \\
\hline \multicolumn{2}{|l|}{ Hydrolysis CEH } \\
\hline Cellulose conversion & $96.3 \%$ \\
\hline Xylan conversion & $98.8 \%$ \\
\hline Arabinan conversion & $98.8 \%$ \\
\hline \multicolumn{2}{|l|}{ Lignin Press } \\
\hline Sugar Recovery (\% inlet) & $95.3 \%$ \\
\hline \multicolumn{2}{|l|}{ Conditioning } \\
\hline Feed Sugar Titer (g/L) & 54.8 \\
\hline Concentrated Sugar Titer $(\mathrm{g} / \mathrm{L})$ & 705.6 \\
\hline
\end{tabular}

\begin{tabular}{|c|c|}
\hline \multicolumn{2}{|l|}{ A500: C4 Acids Fermentation } \\
\hline Contamination Sugar Loss & $3.0 \%$ \\
\hline \multicolumn{2}{|l|}{ Utilization } \\
\hline Glucose & $100.0 \%$ \\
\hline Xylose & $100.0 \%$ \\
\hline Arabinose & $87.4 \%$ \\
\hline Sucrose & $100.0 \%$ \\
\hline \multicolumn{2}{|l|}{ Diversion of substrate to Biomass ( $\%$ of substrate Utilized) } \\
\hline Glucose & $5.0 \%$ \\
\hline Xylose & $15.0 \%$ \\
\hline Arabinose & $2.0 \%$ \\
\hline Sucrose & $5.0 \%$ \\
\hline \multicolumn{2}{|l|}{ Diversion of substrate to Product ( $\%$ of substrate Utilized) } \\
\hline Glucose & $95.0 \%$ \\
\hline Xylose & $85.0 \%$ \\
\hline Arabinose & $85.4 \%$ \\
\hline Sucrose & $95.0 \%$ \\
\hline \multicolumn{2}{|l|}{ Maximum theoretical metabolic yield (g/g) } \\
\hline Glucose to Butyric & 0.489 \\
\hline Glucose to Biomass & 0.820 \\
\hline Xylose to Butyric & 0.489 \\
\hline Xylose to Biomass & 0.820 \\
\hline Arabinose to Butyric & 0.489 \\
\hline Arabinose to Biomass & 0.820 \\
\hline Sucrose to Butyric & 0.489 \\
\hline Sucrose to Biomass & 0.863 \\
\hline \multicolumn{2}{|l|}{ Net Metabolic Yield (g/g substrate consumed) } \\
\hline Glucose to Butyric & 0.4646 \\
\hline Glucose to Biomass & 0.0410 \\
\hline Xylose to Butyric & 0.4156 \\
\hline Xylose to Biomass & 0.1230 \\
\hline Arabinose to Butyric & 0.4176 \\
\hline Arabinose to Biomass & 0.0164 \\
\hline Sucrose to Butyric & 0.4646 \\
\hline Sucrose to Biomass & 0.0432 \\
\hline \multicolumn{2}{|l|}{ Extraction Metrics } \\
\hline HFMC Organic loading (kg Org/kg Aq) & 0.10 \\
\hline Butyric Acid Recovery (\% feed) & 1.00 \\
\hline \multicolumn{2}{|l|}{ A500: Acid Catalytic Upgrading } \\
\hline \multicolumn{2}{|l|}{ Theroetical Yields, Fuel upgading } \\
\hline Acid condensation (g ketone/g acid) & 0.648 \\
\hline Ketone condensation (g enone/g ketone) & 0.9211 \\
\hline Enone hydrogenation (g hydrocarbon/g enone) & 0.943 \\
\hline \multicolumn{2}{|l|}{ Acid Condensation } \\
\hline Temperature (C) & 321.52 \\
\hline Pressure (atm) & 1 \\
\hline Butyric to $4-$ Heptanone $(\mathrm{kg} / \mathrm{kg})$ & 0.65 \\
\hline \multicolumn{2}{|l|}{ Ketone Condensation } \\
\hline Temperature (C) & 180 \\
\hline Pressure (atm) & 11.21 \\
\hline Toluene:Ketone Ratio & 3.63 \\
\hline 4-Heptanone to $\mathrm{C} 13-\mathrm{Enone}(\mathrm{kg} / \mathrm{kg})$ & 0.92 \\
\hline \multicolumn{2}{|l|}{ Enone Hydrogenation } \\
\hline Temperature (C) & 270.00 \\
\hline Pressure (atm) & 35.70 \\
\hline $\mathrm{H} 2$ :Enone ratio (wt:wt) & 0.23 \\
\hline Enone to Hydrocarbon Fuel (kg/kg) & 0.94 \\
\hline
\end{tabular}




\begin{tabular}{|c|c|c|c|}
\hline \multicolumn{2}{|c|}{ A700: Lignin Conditioning and Deconstruction } & \multicolumn{2}{|l|}{ A700: Muconic Acid Fermentation } \\
\hline A700 RL composition & & Production Feed Titer(s) (across average of all) & \\
\hline Total Flow & 22221.7 & Glucose & 8.8 \\
\hline LIGNIN (wt\% total mass) & $30.69 \%$ & Xylose & 12.4 \\
\hline Cellulose (wt $\%$ total mass) & $4.67 \%$ & Arabinose & 4.1 \\
\hline Xylan (wt\% total mass) & $0.77 \%$ & Sucrose & 2.1 \\
\hline Arabinan (wt\% total mass) & $0.07 \%$ & Acetic Acid & 9.9 \\
\hline LgnSol (wt\% total mass) & $0.00 \%$ & Soluble Lignin & 45.4 \\
\hline Solids Flow $(\mathrm{kg} / \mathrm{hr})$ & 14013.67 & Extract & 79.9 \\
\hline A700 BL composition & & $\mathrm{NaOH}$ & 38.2 \\
\hline Total Flow & 92912.1 & Utilization (across average of all) & \\
\hline LIGNIN (wt\% total mass) & $\begin{array}{r}92912.1 \\
664 \%\end{array}$ & Glucose & $98.00 \%$ \\
\hline Cellulose (wt\% total mass) & $0.04 \%$ & Xylose & $98.00 \%$ \\
\hline & $0.63 \%$ & Arabinose & $98.00 \%$ \\
\hline Xylan (wt\% total mass) & $1.75 \%$ & Sucrose & $98.00 \%$ \\
\hline Arabinan (wt\% total mass) & $0.64 \%$ & Acetic Acid & $98.01 \%$ \\
\hline LgnSol (wt\% total mass) & $0.00 \%$ & Soluble Lignin & $98.00 \%$ \\
\hline Glucose (wt $\%$ total mass) & $0.00 \%$ & Extract & $98.00 \%$ \\
\hline Xylose (wt\% total mass) & $0.00 \%$ & Production broth Titer(s) (across average of all) & \\
\hline Arabinose (wt $\%$ total mass) & $0.00 \%$ & Muconic Acid & 68.53 \\
\hline Sucrose (wt\% total mass) & $0.35 \%$ & OLEYEAST & 37.6 \\
\hline Extract (wt\% total mass) & $13.14 \%$ & ZYMO & 1.00 \\
\hline Acetic Acid (wt\% total mass) & $1.62 \%$ & Total DCW & 38.61 \\
\hline Solids Flow (kg/hr) & 11668.91 & $\mathrm{NaOH}$ & 37.2 \\
\hline BL-to BCD & 92912.05 & Clarification & \\
\hline BL-to ferm & 0.014800 & Ultrafilter Muconic Loss & $4.0 \%$ \\
\hline A700 BCD combined feed & & A700: Adipic Acid Upgrading & \\
\hline Total Flow & 115133.7 & Crystallization 1 & \\
\hline LIGNIN (wt\% total mass) & $11.28 \%$ & Muconic Crystalization Temperature & 15.00 \\
\hline Cellulose (wt\% total mass) & $0.90 \%$ & Muconic Acid recovery & $98.7 \%$ \\
\hline Xylan (wt\% total mass) & $0.15 \%$ & Hydrogenation & \\
\hline Arabinan (wt\% total mass) & $0.01 \%$ & Ethanol:Muconic Loading & 4.00 \\
\hline LgnSol (wt\% total mass) & $0.00 \%$ & Hydrogenation Temperature & 78.00 \\
\hline Glucose (wt $\%$ total mass) & $0.81 \%$ & Hydrogenation Pressure (PSI) & 583.00 \\
\hline Xylose (wt\% total mass) & $1.74 \%$ & H2:Muconic Ratio (mol/mol) & 2.673 \\
\hline Arabinose (wt\% total mass) & $0.60 \%$ & Crystallization 2 & \\
\hline Sucrose (wt\% total mass) & $0.28 \%$ & Adipic Crystallization Temperature & 15.00 \\
\hline Extract (wt\% total mass) & $10.60 \%$ & Adipic Acid single pass recovery & $73.4 \%$ \\
\hline Acetic Acid (wt\% total mass) & $1.31 \%$ & & \\
\hline Solids Flow $(\mathrm{kg} / \mathrm{hr})$ & 22876.25 & Waste Water Treatment & \\
\hline Lignin Conditioning and Conversion & & Hydraulic Load (L/hr) & 445024.4 \\
\hline Temperature & 120.00 & Hydraulic Load (gpm) & 1959.4 \\
\hline Pressure (atm) & 5.00 & Hydraulic Load (MMgal/day) & 2.8 \\
\hline $\mathrm{NaOH}$ Titer & 58.07980769 & Total COD $(\mathrm{kg} / \mathrm{hr})$ & 4148.8 \\
\hline & & Total COD (g/L) & 9.3 \\
\hline A700 BCD pulp-outlet & & & \\
\hline Total Flow & 115133.7 & NH3-N in Combined WW mg/L & $\begin{array}{r}66.2 \\
29971\end{array}$ \\
\hline LIGNIN (wt\% total mass) & $5.27 \%$ & $\begin{array}{l}\text { Total BOD (KG/HR) } \\
\text { Total BOD }(\mathrm{g} / \mathrm{L})\end{array}$ & $\begin{array}{r}2987.1 \\
6.7\end{array}$ \\
\hline Cellulose (wt\% total mass) & $0.47 \%$ & NH3-N DEMAND (mg/L) & -1.0 \\
\hline Xylan (wt\% total mass) & $0.08 \%$ & NH3-N ADDITION (KG/HR) & 0.0 \\
\hline Arabinan (wt\% total mass) & $0.01 \%$ & TOTAL Polymer $(\mathrm{kg} / \mathrm{hr})$ & 0.0 \\
\hline LgnSol (wt\% total mass) & $6.02 \%$ & & \\
\hline Glucose (wt $\%$ total mass) & $1.29 \%$ & & \\
\hline Xylose (wt\% total mass) & $1.82 \%$ & & \\
\hline Arabinose (wt\% total mass) & $0.61 \%$ & & \\
\hline Sucrose (wt\% total mass) & $0.28 \%$ & & \\
\hline Extract (wt\% total mass) & $10.60 \%$ & & \\
\hline Acetic Acid (wt\% total mass) & $1.31 \%$ & & \\
\hline Solids Flow $(\mathrm{kg} / \mathrm{hr})$ & 14564.61 & & \\
\hline BCD-SLS & & & \\
\hline solids flow $(\mathrm{kg} / \mathrm{hr})$ & 14564.61 & & \\
\hline entrained water in solids $(\mathrm{kg} / \mathrm{hr})$ & 14564.61 & & \\
\hline liquid flow (kg/hr) & 86004.50 & & \\
\hline Solubilization (across BCD) & & & \\
\hline Cellulose & $47.99 \%$ & & \\
\hline Xylan & $47.99 \%$ & & \\
\hline Arabinan & $47.99 \%$ & & \\
\hline Lignin & $53.33 \%$ & & \\
\hline APL correction reactions & & & \\
\hline Cellulose (wt \% conversion) & $100.00 \%$ & & \\
\hline Cellulose (wt \% degredation) & $0.00 \%$ & & \\
\hline Xylan (wt \% conversion) & $100.00 \%$ & & \\
\hline Xylan (wt \% degredation) & $0.00 \%$ & & \\
\hline Arabinan (wt \% conversion) & $100.00 \%$ & & \\
\hline Arabinan (wt $\%$ degredation) & $0.00 \%$ & & \\
\hline Lignin (wt \% conversion) & $0.00 \%$ & & \\
\hline
\end{tabular}




\begin{tabular}{|lr|}
\hline Final Fuel Product & \\
\hline Fuel Mass flow (kg/hr) & 11031.6 \\
Estimated Density (kg/L) & 0.7 \\
Fuel Volume Flow (L/hr) & 15751.5 \\
Fuel LHV (Mmkcal/hr) & 116.1 \\
Annual Fuel Volume (MM Gal/yr) & 32.8 \\
Annual Fuel GGE (MM GGE/yr) & 31.3 \\
\hline
\end{tabular}

\section{A100: Feedstock}

Moisture Content

Composition (wt\% Dry Feed)

Cellulose

Xylan

Arabinan

Sucrose

Lignin

Acetate

Extract

Ash

Mannan

Galactan

Protein

\section{Hydrolysis Mass yield (g/g biopolymer)}

Cellulose to glucose

Xylan to xylose

Arabinan to arabinose

\section{A200: Deacetylation \& Pretreatment}

\section{Deacetylation Conditions}

Temperature (C)

Pressure (atm)

$\mathrm{NaOH}$ loading $(\mathrm{mg} / \mathrm{L})$

\section{Solubilization to the Black Liquor}

\section{Acetate}

Lignin

Cellulose

Xylan

Arabinan

\begin{tabular}{|lr|}
\hline A300: Whole Slurry Hydrolysis & \\
\hline Operating Conditions & 9.96 \\
Enzyme Loading & \\
Hydrolysis & $91.2 \%$ \\
Cellulose conversion & $90.0 \%$ \\
$\quad$ Xylan conversion & $85.0 \%$ \\
Arabinan conversion & \\
& \\
Residence times (hrs) & 24 \\
Continuous saccharification & 96 \\
Batch saccharification & 36 \\
Batch fermentation & \\
\hline
\end{tabular}

\begin{tabular}{|c|c|}
\hline A500: BDO Fermentation & \\
\hline 2,3,BDO Titer broth & 102.078175 \\
\hline Contamination Sugar Loss & $3.0 \%$ \\
\hline \multicolumn{2}{|l|}{ Utilization } \\
\hline Glucose & $98.1 \%$ \\
\hline Xylose & $92.5 \%$ \\
\hline Arabinose & $89.0 \%$ \\
\hline Sucrose & $95.0 \%$ \\
\hline \multicolumn{2}{|l|}{ Diversion of substrate to Biomass ( $\%$ of substrate Utilized) } \\
\hline Glucose & $2.040 \%$ \\
\hline Xylose & $2.053 \%$ \\
\hline Arabinose & $2.134 \%$ \\
\hline Sucrose & $0.000 \%$ \\
\hline \multicolumn{2}{|l|}{ Diversion of substrate to $2,3-\mathrm{BDO}$ ( $\%$ of substrate Utilized) } \\
\hline Glucose & $96.88 \%$ \\
\hline Xylose & $97.26 \%$ \\
\hline Arabinose & $95.46 \%$ \\
\hline Sucrose & $100.00 \%$ \\
\hline \multicolumn{2}{|l|}{ Maximum theoretical metabolic yield (g/g) } \\
\hline Glucose to 2,3-BDO & 0.500 \\
\hline Glucose to Biomass & 0.820 \\
\hline Xylose to 2,3-BDO & 0.500 \\
\hline Xylose to Biomass & 0.820 \\
\hline Arabinose to $2,3-\mathrm{BDO}$ & 0.500 \\
\hline Arabinose to Biomass & 0.820 \\
\hline Sucrose to 2,3-BDO & 0.527 \\
\hline Sucrose to Biomass & 0.863 \\
\hline \multicolumn{2}{|l|}{ Net Metabolic Yield (g/g substrate consumed) } \\
\hline Glucose to $2,3-\mathrm{BDO}$ & 0.4846 \\
\hline Glucose to Biomass & 0.0167 \\
\hline Xylose to 2,3-BDO & 0.4866 \\
\hline Xylose to Biomass & 0.0168 \\
\hline Arabinose to $2,3-\mathrm{BDO}$ & 0.4776 \\
\hline Arabinose to Biomass & 0.0175 \\
\hline Sucrose to $2,3-\mathrm{BDO}$ & 0.5266 \\
\hline Sucrose to Biomass & 0.0000 \\
\hline \multicolumn{2}{|l|}{ Solid Liquid Separation } \\
\hline 2,3, BDO recovery (\% feed) & $96.42 \%$ \\
\hline \multicolumn{2}{|l|}{ A500: 2,3-BDO Upgrading } \\
\hline \multicolumn{2}{|l|}{ Theroetical Yields, Fuel upgading } \\
\hline 2,3-BDO Dehydration ( $\mathrm{g}$ butene/g BDO) & 0.62257828 \\
\hline Butene Oligomerization (g Oligomer/g Butene) & 1 \\
\hline Oligomer hydrogenation (g Hydrocarbon/g Oligomer) & 1.0359 \\
\hline
\end{tabular}


A700: Lignin Conditioning and Deconstruction

A700 RL composition

Total Flow

LIGNIN (wt\% total mass)

Cellulose (wt\% total mass)

Xylan (wt\% total mass)

Arabinan (wt\% total mass)

LgnSol (wt\% total mass)

Solids Flow $(\mathrm{kg} / \mathrm{hr})$

A700 BL composition

Total Flow

LIGNIN (wt\% total mass)

Cellulose ( $w$ t\% total mass)

Xylan (wt\% total mass)

Arabinan (wt\% total mass)

LgnSol (wt\% total mass)

Glucose (wt\% total mass)

Xylose (wt\% total mass)

Arabinose (wt\% total mass)

Sucrose (wt\% total mass)

Extract (wt\% total mass)

Acetic Acid (wt\% total mass)

Solids Flow $(\mathrm{kg} / \mathrm{hr})$

BL-to BCD

BL-to ferm

A700 BCD combined feed

Total Flow

LIGNIN (wt\% total mass)

Cellulose (wt\% total mass)

Xylan (wt\% total mass)

Arabinan (wt\% total mass)

LgnSol (wt\% total mass)

Glucose (wt\% total mass)

Xylose (wt\% total mass)

Arabinose (wt\% total mass)

Sucrose (wt\% total mass)

Extract (wt\% total mass)

Acetic Acid (wt\% total mass)

Solids Flow ( $\mathrm{kg} / \mathrm{hr}$ )

Lignin Conditioning and Conversion

Temperature

Pressure (atm)

$\mathrm{NaOH}$ Titer

A700 BCD pulp-outlet

Total Flow

LIGNIN (wt\% total mass)

Cellulose (wt\% total mass)

Xylan (wt\% total mass)

Arabinan (wt\% total mass)

LgnSol (wt\% total mass)

Glucose (wt\% total mass)

Xylose (wt\% total mass)

Arabinose (wt\% total mass)

Sucrose (wt\% total mass)

Extract (wt\% total mass)

Acetic Acid (wt\% total mass)

Solids Flow $(\mathrm{kg} / \mathrm{hr})$

BCD-SLS

solids flow $(\mathrm{kg} / \mathrm{hr})$

entrained water in solids $(\mathrm{kg} / \mathrm{hr})$

liquid flow $(\mathrm{kg} / \mathrm{hr})$

Solubilization (across BCD)

Cellulose

Xylan

Arabinan

Lignin

APL correction reactions

Cellulose (wt \% conversion)

Cellulose (wt \% degredation)

Xylan (wt \% conversion)

Xylan (wt \% degredation)

Arabinan (wt \% conversion)

Arabinan (wt \% degredation)

Lignin (wt \% conversion)
A700: Muconic Acid Fermentation

Production Feed Titer(s) (across average of all)

24928.0

$27.36 \%$

$9.90 \%$

$5.76 \%$

$0.82 \%$

$0.00 \%$

17822.45

92912.1

$6.64 \%$

$0.63 \%$

$1.75 \%$

$0.64 \%$

$0.00 \%$

$0.00 \%$

$0.00 \%$

$0.00 \%$

$0.35 \%$

$13.14 \%$

$1.62 \%$

11668.91

92912.05

0.014800

117840.1

$11.03 \%$

$2.09 \%$

$1.22 \%$

$0.17 \%$

$0.00 \%$

$0.57 \%$

$1.60 \%$

$0.58 \%$

$0.27 \%$

$10.36 \%$

$1.28 \%$

26685.02

120.00

5.00

57.83561938

117840.1

$5.15 \%$

$5.15 \%$
$1.09 \%$

$0.63 \%$

$0.09 \%$

$5.88 \%$

$1.68 \%$

$2.27 \%$

$0.67 \%$

$0.27 \%$

$10.36 \%$

$1.28 \%$

16989.85

16989.85

16989.85

83860.37

$47.99 \%$

$47.99 \%$

$47.99 \%$

$53.33 \%$

$100.00 \%$

$0.00 \%$

$100.00 \%$

$0.00 \%$

$100.00 \%$

$0.00 \%$ $0.00 \%$
Arabinose

Sucrose

Acetic Acid

Soluble Lignin

Extract

$\mathrm{NaOH}$

Utilization (across average of all)

Glucose

Xylose

Arabinose

Sucrose

Acetic Acid

Soluble Lignin

Extract

Production broth Titer(s) (across average of all)

Muconic Acid

OLEYEAST

ZYMO

Total DCW

$\mathrm{NaOH}$

Clarification

\begin{tabular}{ll} 
Ultrafilter Muconic Loss & $4.3 \%$ \\
\hline
\end{tabular}

A700: Adipic Acid Upgrading

Crystallization 1

\begin{tabular}{lr} 
Muconic Crystalization Temperature & 15.00 \\
\hline$u c o n i c$ & $98.8 \%$
\end{tabular}

Muconic Acid recovery

Hydrogenation

Ethanol:Muconic Loading

Hydrogenation Temperature

Hydrogenation Pressure (PSI)

$\mathrm{H} 2$ :Muconic Ratio ( $\mathrm{mol} / \mathrm{mol}$ )

Crystallization 2

Adipic Crystallization Temperature

Adipic Acid single pass recovery

Waste Water Treatment

Hydraulic Load (L/hr)

Hydraulic Load (gpm)

Hydraulic Load (MMgal/day)

Total COD $(\mathrm{kg} / \mathrm{hr})$

Total COD (g/L)

NH3-N in Combined WW mg/L

Total BOD (KG/HR)

Total BOD $(\mathrm{g} / \mathrm{L})$

NH3-N DEMAND (mg/L)

NH3-N ADDITION (KG/HR)

TOTAL Polymer $(\mathrm{kg} / \mathrm{hr})$ 


\section{Appendix D. Key Aspen Stream Data Tables}

High-level stream table information from Aspen Plus modeling output follows, for key streams associated with each process operation area under both pathway scenarios. Space for stream tables was limited; below is a key to lumped components. As the stream table information focuses primarily on the high-level overall process and does not include every individual modeled stream within each process area, mass balance closure around a given unit area may not be $100 \%$.

Other sugars (SS)

Other organic acids (SS)

Fermentation nutrients (SS)

Other chemicals (SS)

Solvent

$\mathrm{CO} / \mathrm{SO}_{\mathrm{X}} / \mathrm{NO}_{\mathrm{X}} / \mathrm{H}_{2} \mathrm{~S}$

Other structural carbohydrates (IS)

Protein (IS)

Cell mass (IS)

Other insoluble solids (IS)
Arabinose, mannose, galactose, sucrose

Acetic, butyric, muconic, and lactic acids, extractives

Sodium hydroxide, CSL, DAP, other minor nutrients

Ammonia, ammonium sulfate, sodium sulfate, sodium nitrate

TOA, toluene, ethanol

$\mathrm{NO}, \mathrm{NO}_{2}, \mathrm{SO}_{2}, \mathrm{CO}, \mathrm{H}_{2} \mathrm{~S}$

Xylan, arabinan, galactan, mannan

Corn protein, enzymes, denatured enzymes

Cell biomass for fermentation, enzyme, and wastewater organisms

Ash, lime, calcium sulfate 


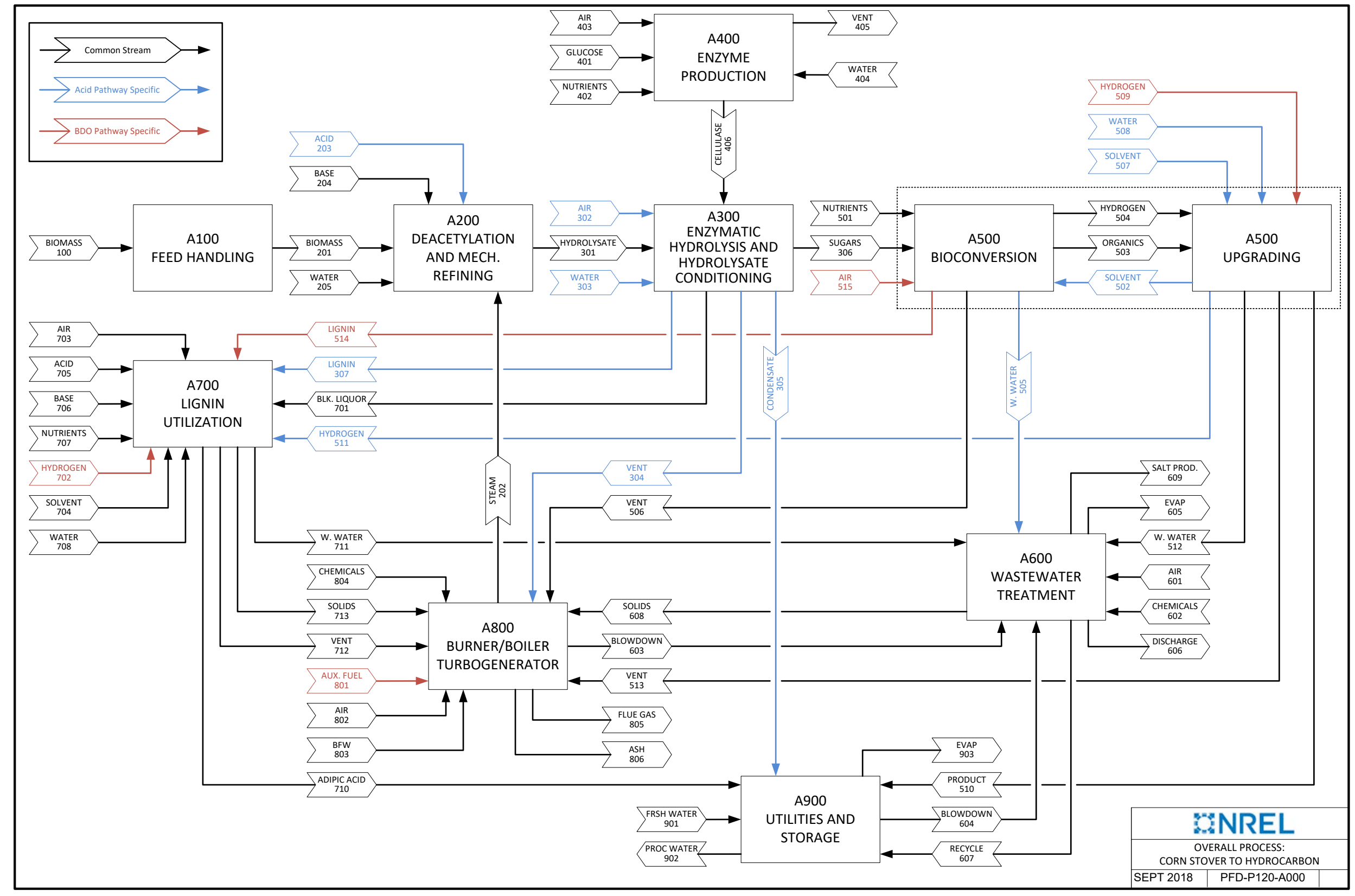




\section{Acids Pathway}

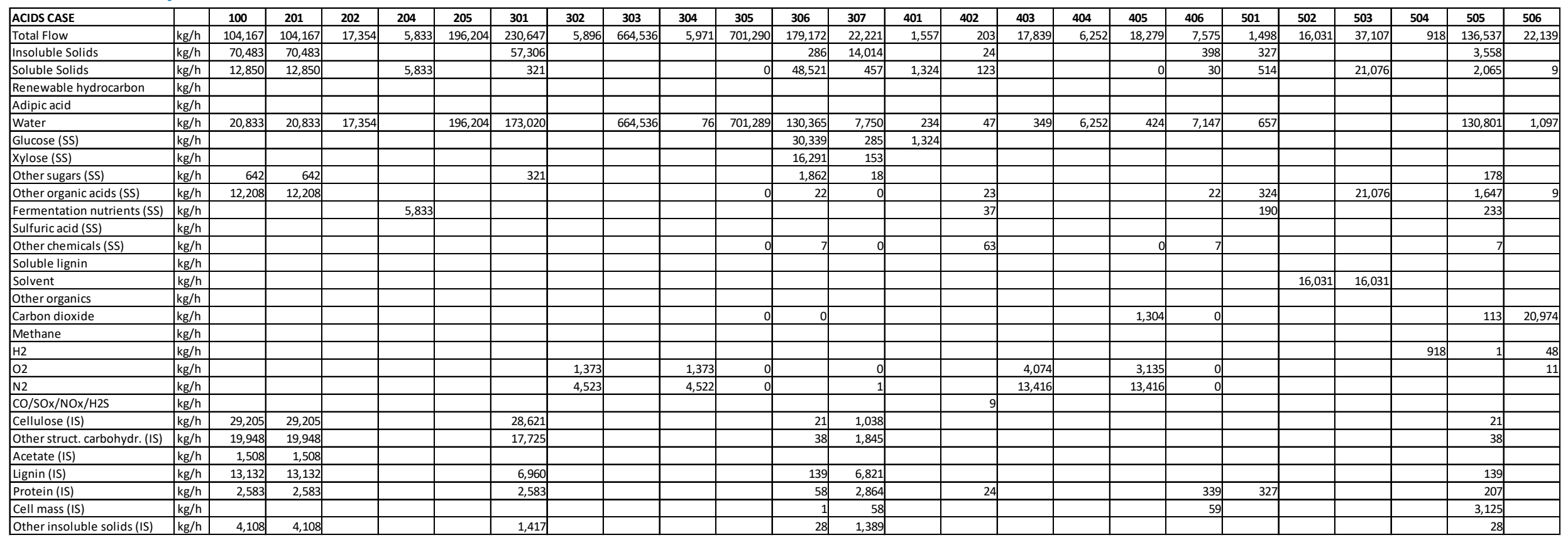

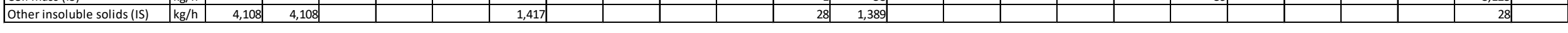

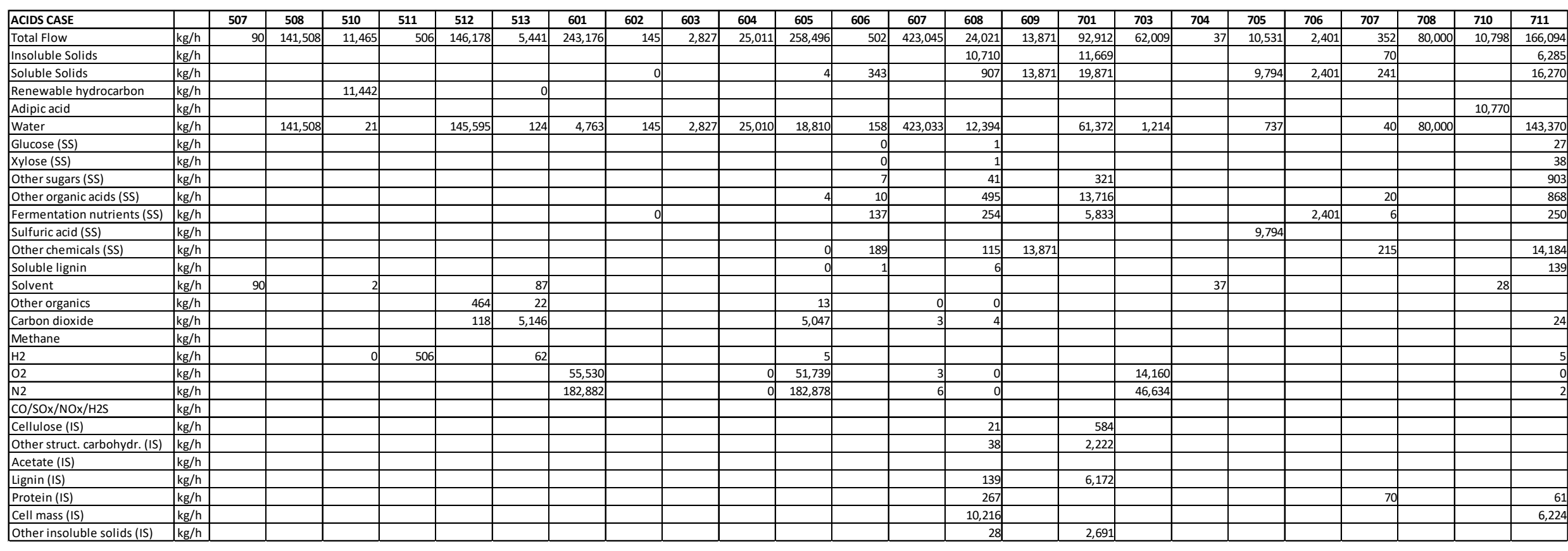




\begin{tabular}{|c|c|c|c|c|c|c|c|c|c|c|c|}
\hline ACIDS CASE & & 712 & 713 & 802 & 803 & 804 & 805 & 806 & 901 & 902 & 903 \\
\hline Total Flow & $\mathrm{kg} / \mathrm{h}$ & 64,949 & 29,129 & 167,672 & 24,353 & 1,699 & 320,454 & 4,270 & 209,901 & $1,113,390$ & 195,823 \\
\hline Insoluble Solids & $\mathrm{kg} / \mathrm{h}$ & & 14,565 & & & 97 & & 4,270 & & & \\
\hline Soluble Solids & $\mathrm{kg} / \mathrm{h}$ & 2 & & & & 1,215 & 367 & & & & \\
\hline Renewable hydrocarbon & $\mathrm{kg} / \mathrm{h}$ & & & & & & & & & & \\
\hline Adipic acid & $\mathrm{kg} / \mathrm{h}$ & & & & & & & & & & \\
\hline Water & $\mathrm{kg} / \mathrm{h}$ & 2,111 & 14,565 & 3,284 & 24,353 & 388 & 54,908 & & 209,901 & $1,113,390$ & 195,823 \\
\hline Glucose (SS) & $\mathrm{kg} / \mathrm{h}$ & & & & & & & & & & \\
\hline Xylose (SS) & $\mathrm{kg} / \mathrm{h}$ & & & & & & & & & & \\
\hline Other sugars (SS) & $\mathrm{kg} / \mathrm{h}$ & & & & & & & & & & \\
\hline Other organic acids (SS) & $\mathrm{kg} / \mathrm{h}$ & 2 & & & & & & & & & \\
\hline Fermentation nutrients (SS) & $\mathrm{kg} / \mathrm{h}$ & & & & & & 252 & & & & \\
\hline Sulfuric acid (SS) & $\mathrm{kg} / \mathrm{h}$ & & & & & & & & & & \\
\hline Other chemicals (SS) & $\mathrm{kg} / \mathrm{h}$ & & & & & 1,215 & 115 & & & & \\
\hline Soluble lignin & $\mathrm{kg} / \mathrm{h}$ & & & & & & & & & & \\
\hline \begin{tabular}{|l|} 
Solvent \\
\end{tabular} & $\mathrm{kg} / \mathrm{h}$ & 8 & & & & & & & & & \\
\hline Other organics & $\mathrm{kg} / \mathrm{h}$ & & & & & & & & & & \\
\hline Carbon dioxide & $\mathrm{kg} / \mathrm{h}$ & 10,245 & & & & & 77,814 & & & & \\
\hline Methane & $\mathrm{kg} / \mathrm{h}$ & & & & & & & & & & \\
\hline $\mathrm{H} 2$ & $\mathrm{~kg} / \mathrm{h}$ & 204 & & & & & & & & & \\
\hline 02 & $\mathrm{~kg} / \mathrm{h}$ & 5,744 & & 38,289 & & & 7,553 & & & & \\
\hline $\mathrm{N} 2$ & $\mathrm{~kg} / \mathrm{h}$ & 46,633 & & 126,100 & & & 179,231 & & & & \\
\hline $\mathrm{CO} / \mathrm{SO} / \mathrm{NOx} / \mathrm{H} 2 \mathrm{~S}$ & $\mathrm{~kg} / \mathrm{h}$ & & & & & & 580 & & & & \\
\hline \begin{tabular}{|l|} 
Cellulose (IS) \\
\end{tabular} & $\mathrm{kg} / \mathrm{h}$ & & 540 & & & & & & & & \\
\hline Other struct. carbohydr. (IS) & $\mathrm{kg} / \mathrm{h}$ & & 959 & & & & & & & & \\
\hline Acetate (IS) & $\mathrm{kg} / \mathrm{h}$ & & & & & & & & & & \\
\hline Lignin (IS) & $\mathrm{kg} / \mathrm{h}$ & & 6,064 & & & & & & & & \\
\hline Protein (IS) & $\mathrm{kg} / \mathrm{h}$ & & 2,864 & & & & & & & & \\
\hline Cell mass (IS) & $\mathrm{kg} / \mathrm{h}$ & & 58 & & & & & & & & \\
\hline Other insoluble solids (IS) & $\mathrm{kg} / \mathrm{h}$ & & 4,080 & & & 97| & & 4,270 & & & \\
\hline
\end{tabular}




\section{BDO Pathway}

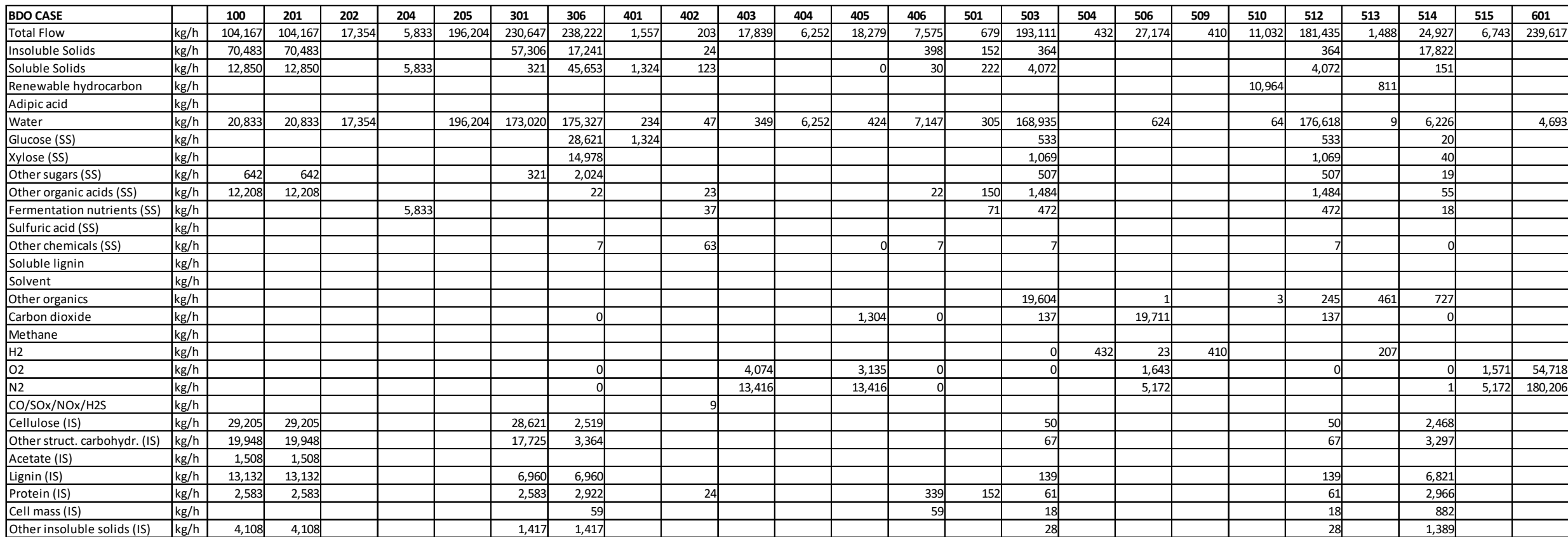

\begin{tabular}{|l|l|l|l|l|}
\hline Other insoluble solids (IS) & $\mathrm{kg} / \mathrm{h}$ & 4,108 & 4,108 \\
\hline
\end{tabular}

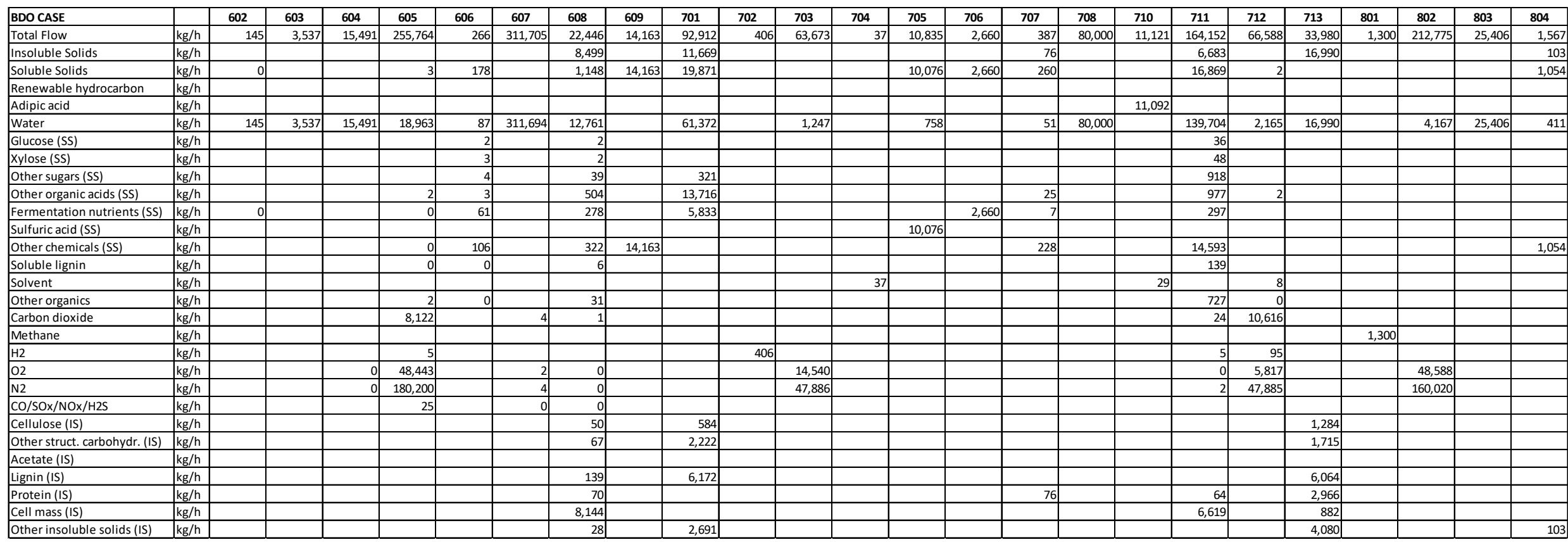

\begin{tabular}{|l|l|}
\hline Other insoluble solids (IS) & $\mathrm{kg} / \mathrm{h}$
\end{tabular} 


\begin{tabular}{|l|l|c|c|c|c|c|}
\hline BDO CASE & & \multicolumn{1}{|c|}{805} & \multicolumn{1}{c|}{806} & \multicolumn{1}{c|}{901} & \multicolumn{1}{c|}{902} & \multicolumn{1}{c|}{903} \\
\hline Total Flow & $\mathrm{kg} / \mathrm{h}$ & 367,036 & 4,279 & 133,396 & 308,422 & 121,177 \\
\hline Insoluble Solids & $\mathrm{kg} / \mathrm{h}$ & & 4,279 & & & \\
\hline Soluble Solids & $\mathrm{kg} / \mathrm{h}$ & 599 & & & & \\
\hline Renewable hydrocarbon & $\mathrm{kg} / \mathrm{h}$ & & & & & \\
\hline Adipic acid & $\mathrm{kg} / \mathrm{h}$ & & & & & \\
\hline Water & $\mathrm{kg} / \mathrm{h}$ & 62,839 & & 133,396 & 308,422 & 121,177 \\
\hline Glucose (SS) & $\mathrm{kg} / \mathrm{h}$ & & & & & \\
\hline Xylose (SS) & $\mathrm{kg} / \mathrm{h}$ & & & & & \\
\hline Other sugars (SS) & $\mathrm{kg} / \mathrm{h}$ & & & & & \\
\hline Other organic acids (SS) & $\mathrm{kg} / \mathrm{h}$ & & & & & \\
\hline Fermentation nutrients (SS) & $\mathrm{kg} / \mathrm{h}$ & 277 & & & & \\
\hline Sulfuric acid (SS) & $\mathrm{kg} / \mathrm{h}$ & & & & & \\
\hline Other chemicals (SS) & $\mathrm{kg} / \mathrm{h}$ & 322 & & & & \\
\hline Soluble lignin & $\mathrm{kg} / \mathrm{h}$ & & & & & \\
\hline Solvent & $\mathrm{kg} / \mathrm{h}$ & & & & & \\
\hline Other organics & $\mathrm{kg} / \mathrm{h}$ & & & & & \\
\hline Carbon dioxide & $\mathrm{kg} / \mathrm{h}$ & 78,971 & & & & \\
\hline Methane & $\mathrm{kg} / \mathrm{h}$ & & & & & \\
\hline H2 & $\mathrm{kg} / \mathrm{h}$ & & & & & \\
\hline O2 & $\mathrm{kg} / \mathrm{h}$ & 9,324 & & & & \\
\hline N2 & $\mathrm{kg} / \mathrm{h}$ & 214,782 & & & & \\
\hline CO/SOx/NOx/H2S & $\mathrm{kg} / \mathrm{h}$ & 521 & & & & \\
\hline Cellulose (IS) & $\mathrm{kg} / \mathrm{h}$ & & & & & \\
\hline Other struct. carbohydr. (IS) & $\mathrm{kg} / \mathrm{h}$ & & & & & \\
\hline Acetate (IS) & $\mathrm{kg} / \mathrm{h}$ & & & & & \\
\hline Lignin (IS) & $\mathrm{kg} / \mathrm{h}$ & & & & & \\
\hline Protein (IS) & $\mathrm{kg} / \mathrm{h}$ & & & & & \\
\hline Cell mass (IS) & $\mathrm{kg} / \mathrm{h}$ & & & & & \\
\hline Other insoluble solids (IS) & $\mathrm{kg} / \mathrm{h}$ & & 4,279 & & & \\
\hline
\end{tabular}

